\title{
Fe-catalyzed reductive couplings of terminal (hetero)aryl alkenes and alkyl halides under aqueous micellar conditions
}

\author{
Haobo Pang, Ye Wang, Fabrice Gallou, and Bruce H. Lipshutz* \\ Department of Chemistry \& Biochemistry \\ University of California \\ Santa Barbara, CA 93106 USA \\ *lipshutz@chem.ucsb.edu
}

\section{Supporting Information}

1. General Experimental Details 2

2. Optimization 3

3. Optimized General Procedure for Coupling Reactions 9

4. Preparation of substrates 10

5. Selectivity on 1-phenylbutadiene 14

6. E Factor and Recycle Studies $\quad 15$

7. Reaction at the gram scale 16

8. Synthesis of the precursor to a SphK inhibitor 17

9. Mechanistic studies $\quad 18$

10. Analytical data for products 27

11. References

12. ${ }^{1} \mathrm{H}$ NMR and ${ }^{13} \mathrm{C}$ NMR spectra $\quad 59$ 


\section{General Experimental Details}

All manipulations were carried out under air unless otherwise noted. TLC plates (UV 254 indicator, glass backed, thickness $200 \mathrm{~mm}$ and silica gel (standard grade, 230 - $400 \mathrm{mesh}$ ) were purchased from Merck. Ethyl acetate and hexanes were purchased from Fisher Scientific. THF was taken from Innovative Technologies Solvent Purification System (SPS) and used immediately. Ligands were either purchased from Sigma-Aldrich or gifted by Johnson Matthey. Pure NMR solvents were purchased from Cambridge Isotopes Laboratories. The coupling reactions were performed in $4 \mathrm{~mL}$ close cap microwave vials either under argon atmosphere or air conditions. Microwave reaction vials were either made in the UCSB glass shop or purchased from VWR international. Reaction vials were also recycled and re-used. Aqueous solution of TPGS-750-M was well purged with argon before use. HPLC grade water was used to prepare surfactant solution. TPGS-750-M was synthesized according to the published procedure ${ }^{1}$ and is also commercially available from Sigma-Aldrich (catalog number 733857). Unless otherwise mentioned, all NMR spectra were recorded at $23{ }^{\circ} \mathrm{C}$ on Varian Unity INOVA $(400,500$ and $600 \mathrm{MHz})$ spectrometers. Reported chemical shifts are referenced to residual solvent peaks. ${ }^{2}$ IR spectra were acquired on a FTIR Perkin Elmer Spectrum Two: UATR Two spectrometer using $1 \mathrm{~cm}^{-1}$ resolution. High resolution mass analyses were obtained using a 5975C Mass Selective Detector, coupled with a 7890A Gas Chromatograph (Agilent Technologies). As capillary column a HP-5MS cross-linked 5\% phenylmethylpolysiloxanediphenyl column $(30 \mathrm{~m} \times 0.250 \mathrm{~mm}, 0.25$ micron, Agilent Technologies $)$ was employed. Helium was used as carrier gas at a constant flow of $1 \mathrm{~mL} / \mathrm{min}$. 


\section{Optimization}

Table S1 Initial finding and screening of metal source

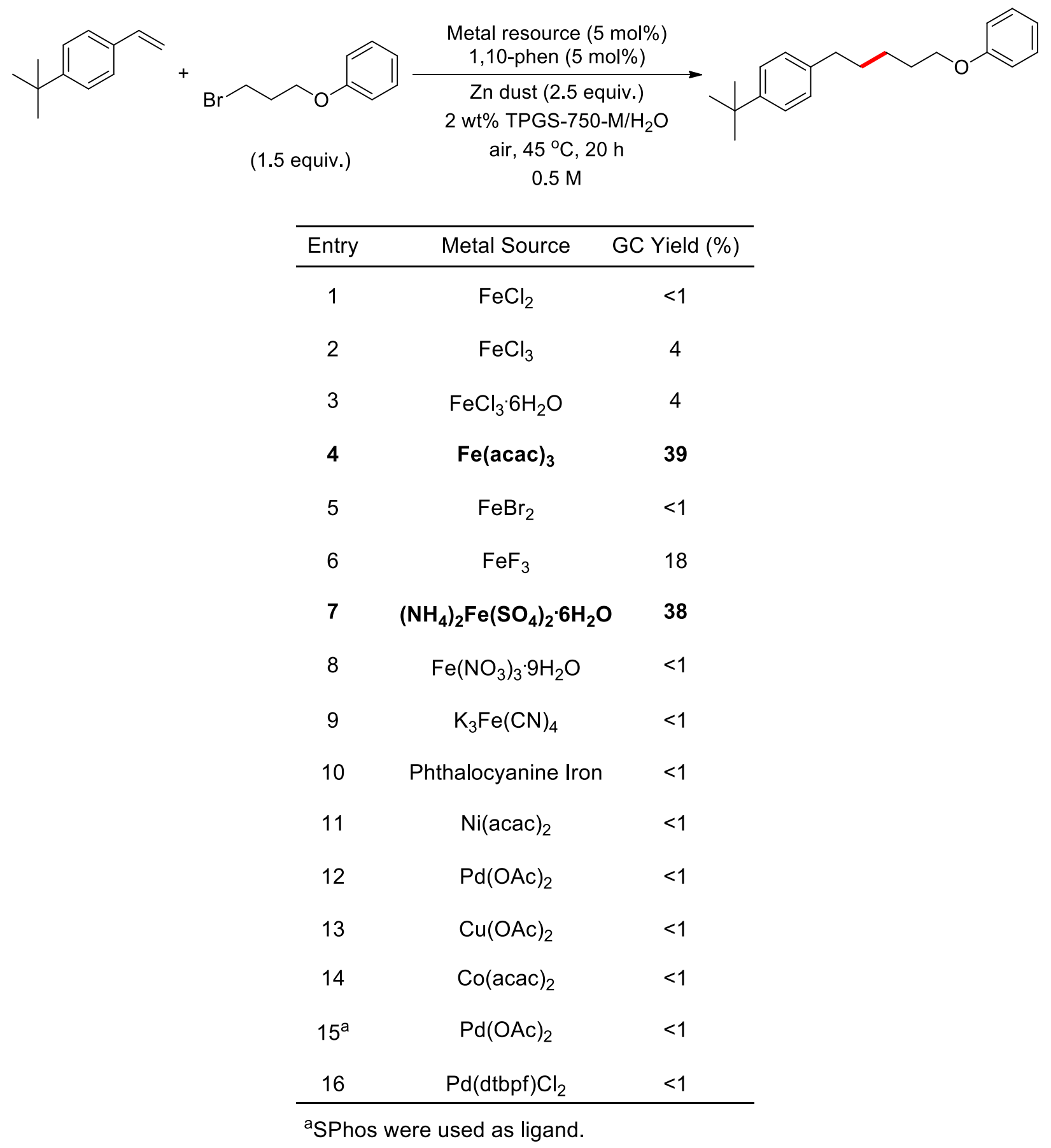


Table S2 Ligand screening
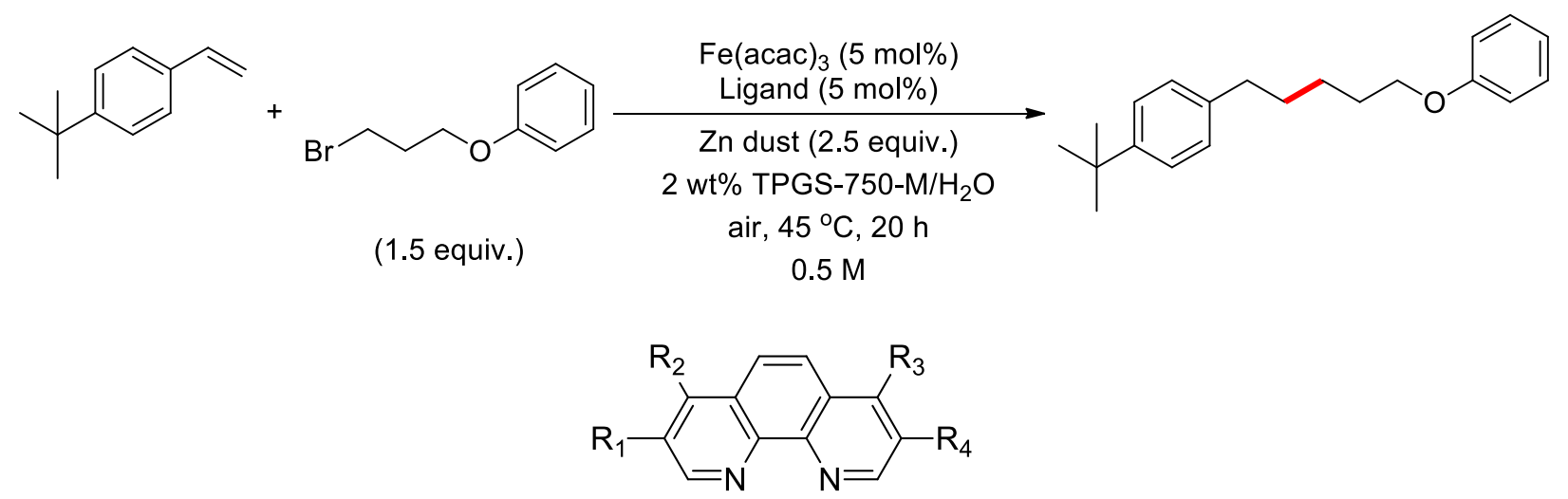

L1: $\mathrm{R}_{1}, \mathrm{R}_{2}, \mathrm{R}_{3}, \mathrm{R}_{4}=\mathrm{H}$

L2: $R_{1}, R_{4}=H, R_{2}, R_{3}=P h$

L3: $R_{1}, R_{4}=H, R_{2}, R_{3}=C l$

L4: $R_{1}, R_{4}=H, R_{2}, R_{3}=N_{2}$

L5: $\mathrm{R}_{1}, \mathrm{R}_{2}, \mathrm{R}_{3}, \mathrm{R}_{4}=\mathrm{Me}$

\begin{tabular}{ccc}
\hline Entry & Ligand & GC Yield (\%) \\
\hline 1 & - & 7 \\
2 & PPh $_{3}$ & 18 \\
3 & SPhos & 20 \\
4 & $2,2^{\prime}-$ bipy & 22 \\
5 & TMEDA & $<1$ \\
6 & DMEDA & $<1$ \\
7 & Tripyridine & 14 \\
8 & L1 (1,10-phen $)$ & 39 \\
9 & L2 & 47 \\
10 & L3 & $<1$ \\
11 & L4 & 28 \\
12 & L5 & $\mathbf{5 5}$ \\
13 & L5 (10 mol\%) & 27 \\
\hline
\end{tabular}


Table S3 Variation in reaction conditions (relative amounts of reactants and reagents)

\begin{tabular}{|c|c|c|c|c|}
\hline A & \multicolumn{3}{|c|}{$\begin{array}{c}\mathrm{Fe}(\mathrm{acac})_{3}, \mathrm{~L} 5, \mathrm{Zn} \text { dust } \\
2 \mathrm{wt} \% \mathrm{TPGS}-750-\mathrm{M} / \mathrm{H}_{2} \mathrm{O} \\
\text { air, } 45^{\circ} \mathrm{C}, 20 \mathrm{~h} \\
0.5 \mathrm{M}\end{array}$} & \multirow[b]{2}{*}{ GC Yield (\%) } \\
\hline Entry & $\mathrm{Fe}(\mathrm{acac})_{3} / \mathrm{L} 5(\mathrm{~mol} \%)$ & B (equiv.) & Zn dust (equiv.) & \\
\hline 1 & $5 / 5$ & 1.5 & 2.5 & 55 \\
\hline 2 & $3 / 3$ & 1.5 & 2.5 & 54 \\
\hline 3 & $2 / 2$ & 1.5 & 2.5 & 24 \\
\hline 4 & $1 / 1$ & 1.5 & 2.5 & 23 \\
\hline 5 & $2 / 2$ & 3 & 2.5 & 59 \\
\hline 6 & $2 / 2$ & 3 & 4.5 & 62 \\
\hline 7 & $2 / 2$ & 3 & 6 & 70 \\
\hline 8 & $3 / 3$ & 3 & 8 & 35 \\
\hline 9 & $2 / 2$ & 3 & 8 & 69 \\
\hline
\end{tabular}


Table S4 Variation in reaction conditions (surfactant and concentration)

\begin{tabular}{|c|c|c|c|}
\hline \multicolumn{3}{|c|}{$\begin{array}{c}\mathrm{Fe}(\mathrm{acac})_{3}(2 \mathrm{~mol} \%) \\
\text { L5 }(2 \mathrm{~mol} \%)\end{array}$} & \multirow[b]{2}{*}{ GC Yield (\%) } \\
\hline Entry & Surfactant & Conc. of reaction & \\
\hline 1 & TPGS-750-M (2 wt\%) & $0.5 \mathrm{M}$ & 72 \\
\hline 2 & pure water & $0.5 \mathrm{M}$ & 64 \\
\hline 3 & TPGS-750-M (1 wt\%) & $0.5 \mathrm{M}$ & 69 \\
\hline 4 & TPGS-750-M (4 wt\%) & $0.5 \mathrm{M}$ & 61 \\
\hline 5 & NOK (2 wt\%) & $0.5 \mathrm{M}$ & 70 \\
\hline 6 & Brij-30 (2 wt\%) & $0.5 \mathrm{M}$ & 69 \\
\hline 7 & TPGS-750-M (2 wt\%) & $0.25 \mathrm{M}$ & 48 \\
\hline 8 & TPGS-750-M (2 wt\%) & $1 \mathrm{M}$ & 70 \\
\hline
\end{tabular}


Table S5 Variation in reaction conditions (salt additive and co-solvent)

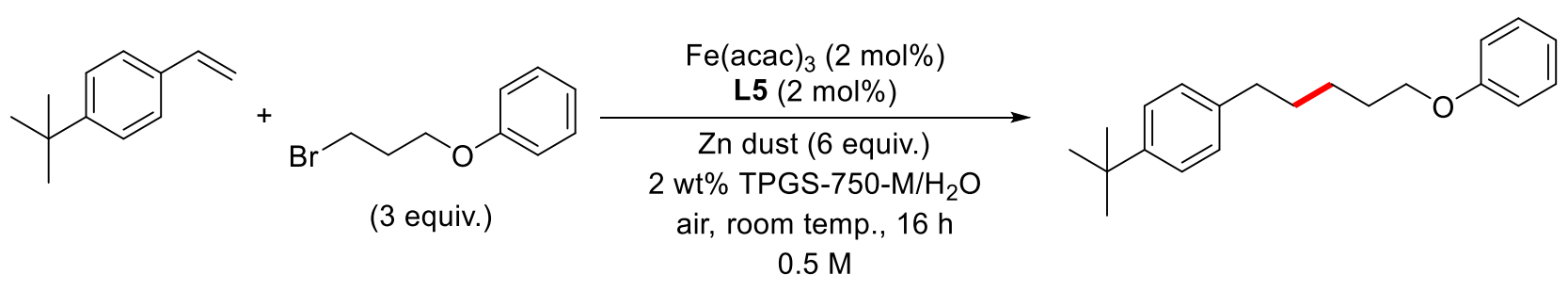

\begin{tabular}{|c|c|c|c|}
\hline Entry & Salt additive (equiv.) & Co-solvent (v/v\%) & GC Yield (\%) \\
\hline 1 & - & - & 72 \\
\hline 2 & $\mathrm{NaCl}(3)$ & - & 76 \\
\hline 3 & $\mathrm{NaCl}(6)$ & - & 74 \\
\hline 4 & $\mathrm{NH}_{4} \mathrm{Cl}(0.5)$ & - & 60 \\
\hline 5 & $\mathrm{NH}_{4} \mathrm{Cl}(1)$ & - & 42 \\
\hline 6 & $\mathrm{NaOAc}(0.5)$ & - & 68 \\
\hline $7^{a}$ & $\mathrm{NaOAc}(0.5)$ & - & $78\left(73^{b}\right)$ \\
\hline $8^{a}$ & $\mathrm{NaOAc}(1)$ & - & 77 \\
\hline $9^{a}$ & $\mathrm{NaOAc}(2)$ & - & 72 \\
\hline $10^{a}$ & $\mathrm{NaOAc}(0.5)$ & THF (10) & 72 \\
\hline $11^{\mathrm{a}}$ & $\mathrm{NaOAc}(0.5)$ & THF (20) & 53 \\
\hline $12^{\mathrm{a}}$ & $\mathrm{NaOAc}(0.5)$ & THF (50) & 8 \\
\hline $13^{a}$ & $\mathrm{NaOAc}(0.5)$ & Acetone (10) & 68 \\
\hline $14^{a}$ & NaOAc (0.5) & $\operatorname{MeCN}(10)$ & $81\left(75^{b}\right)$ \\
\hline $15^{\mathrm{a}}$ & $\mathrm{NaOAc}(0.5)$ & $\operatorname{MeCN}(2.5)$ & 33 \\
\hline $16^{a}$ & $\mathrm{NaOAc}(0.5)$ & $\mathrm{MeCN}(5)$ & 57 \\
\hline $17^{a}$ & $\mathrm{NaOAc}(0.5)$ & $\mathrm{MeOH}(10)$ & 75 \\
\hline $18^{a}$ & $\mathrm{NaOAc}(0.5)$ & $\operatorname{MeCN}(20)$ & 64 \\
\hline
\end{tabular}

${ }^{\mathrm{a}}\left(\mathrm{NH}_{4}\right)_{2} \mathrm{Fe}\left(\mathrm{SO}_{4}\right)_{2} \cdot 6 \mathrm{H}_{2} \mathrm{O}$ was used instead of $\mathrm{Fe}(\mathrm{acac})_{3}$. ${ }^{\mathrm{b}}$ Isolated Yield. 
Table S6 Variation in reaction conditions (other conditions)

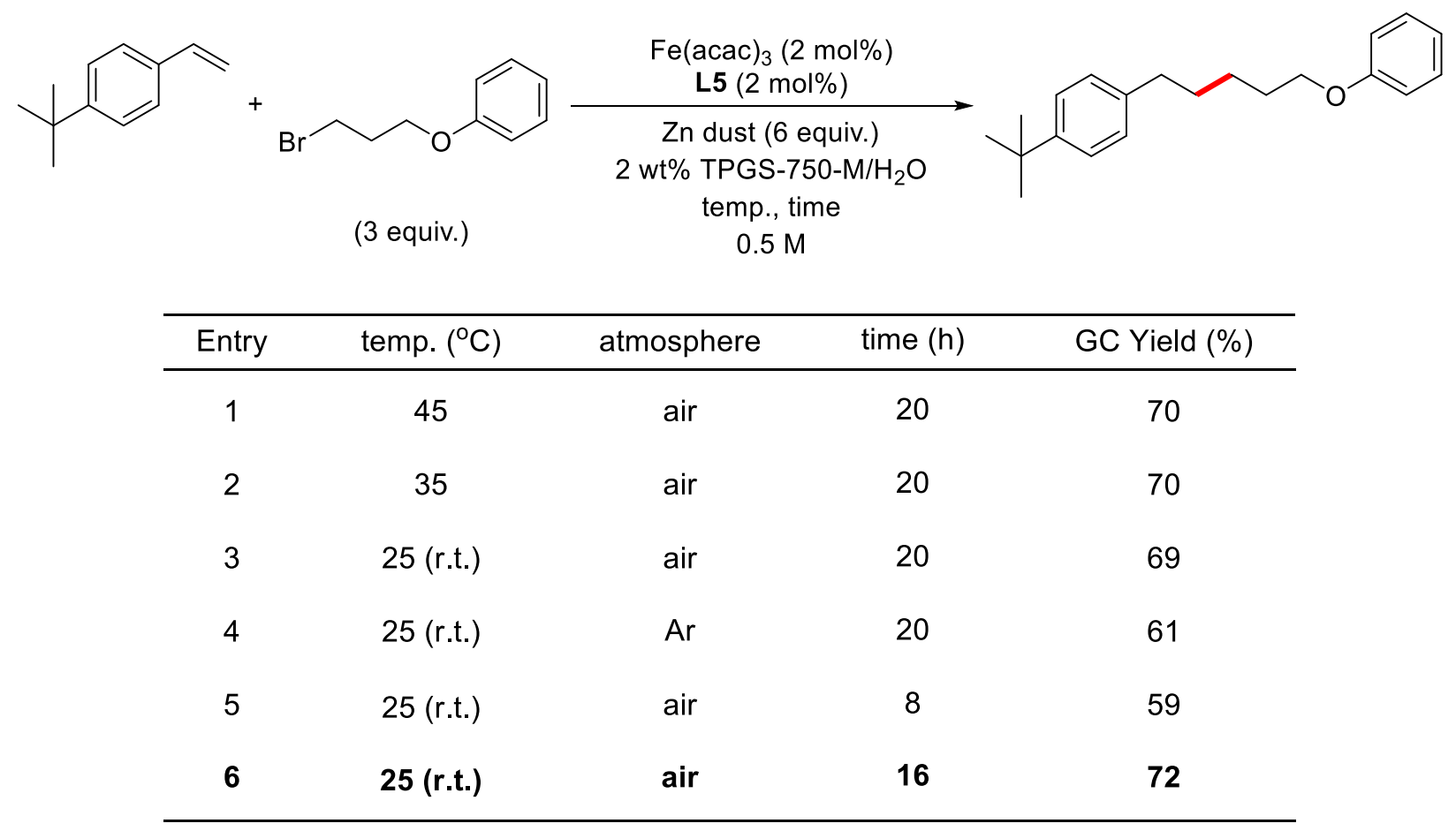

Table S7 Grade of Zinc and zinc equivalent

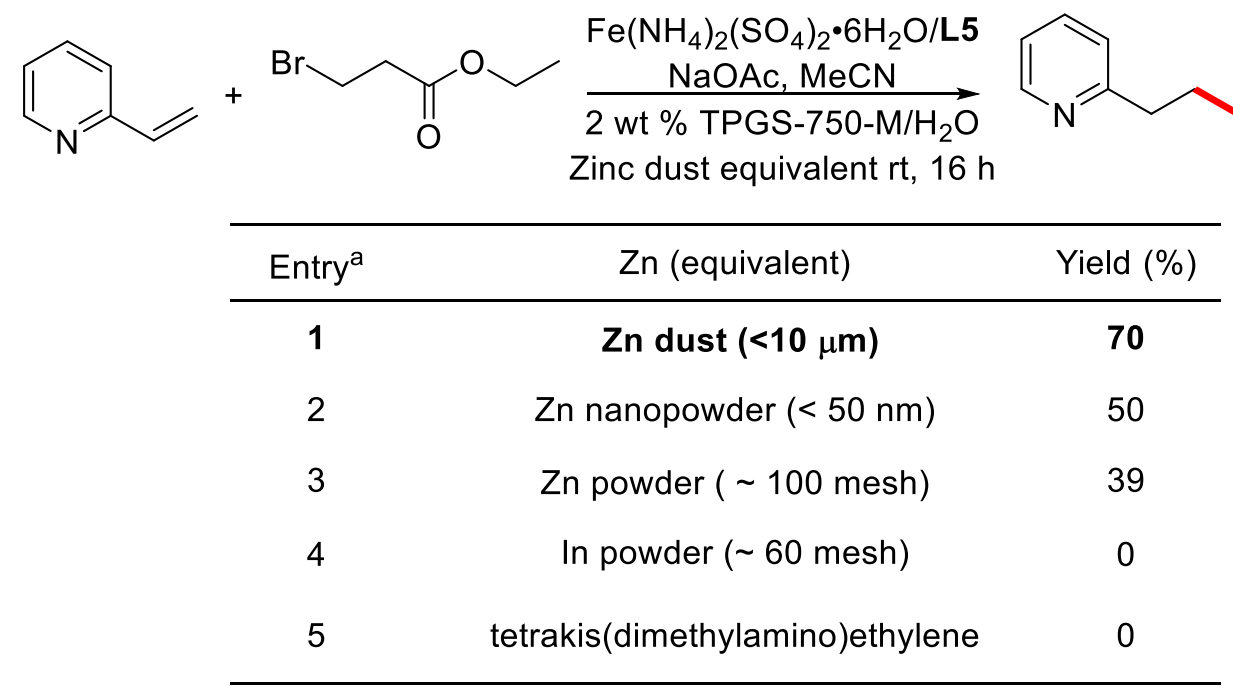

${ }^{a}$ Reaction conditions: olefin $(0.2 \mathrm{mmol}, 1$ equiv), aliphatic bromide (0.6 mmol), $\mathrm{Fe}\left(\mathrm{NH}_{4}\right)_{2}\left(\mathrm{SO}_{4}\right)_{2} \cdot 6 \mathrm{H}_{2} \mathrm{O}(2 \mathrm{~mol} \%)$, $\mathbf{L 5}(2 \mathrm{~mol} \%), \mathrm{Zn}$ or equivalent (6 equiv), $\mathrm{AcONa}(0.1 \mathrm{mmol}), \mathrm{MeCN}(0.04 \mathrm{~mL})$ and $2 \mathrm{wt}$ $\%$ TPGS-750-M/ $\mathrm{H}_{2} \mathrm{O}(0.4 \mathrm{~mL})$. 


\section{Optimized General Procedure for Coupling Reactions}

Procedure. In a flame dried $4 \mathrm{~mL}$ microwave reaction vial, $\mathrm{Fe}(\mathrm{acac})_{3}(1.4 \mathrm{mg}, 0.004 \mathrm{mmol}), 3,4,7,8$ tetramethyl-1,10-phenanthroline (1 mg, $0.004 \mathrm{mmol})$ and $\mathrm{NaCl}(70.2 \mathrm{mg}, 1.2 \mathrm{mmol})$ were mixed together. Then, $0.4 \mathrm{~mL}$ of an aqueous solution $2 \mathrm{wt} \%$ TPGS-750-M was added to the vial followed by sequential addition of olefin ( $0.2 \mathrm{mmol}, 1.0$ equiv) and $\mathrm{Zn}$ dust ( $<10 \mu \mathrm{m}, \geq 98 \%$ from Sigma-Aldrich) (1.2 mmol, 6 equiv). After $5 \mathrm{~min}$ of stirring, the aliphatic bromide ( $0.6 \mathrm{mmol}, 3$ equiv) was added and the reaction vial was closed with a rubber septum under air. The reaction mixture was stirred at $25{ }^{\circ} \mathrm{C}$ for $16 \mathrm{~h}$.

For screening experiments, EtOAc $(1.0 \mathrm{~mL})$ was added to the reaction mixture, and the mixture was stirred

for $5 \mathrm{~min}$ at rt. Stirring was then stopped and the organic layer was removed with a pipette. It was then passed through a very small silica plug. GC yields were determined by GC-MS using naphthalene as internal standard. After the reaction, Zn complex would form agglomeration attach the vial and stir bar, which usually occur in the $\mathrm{Zn}$ reaction. Additional research was needed to approve that problem.

For isolation of product, after $16 \mathrm{~h}$ of reaction, the mixture was extracted with EtOAc $(0.2 \mathrm{~mL} \times 3)$ with the help of centrifuge to effect phase separation. The combined organic extracts were dried over anhydrous $\mathrm{Na}_{2} \mathrm{SO}_{4}$. Volatiles were removed under reduced pressure to obtain crude product which were further purified by flash chromatography over silica gel using EtOAc/hexanes as eluent.

Altered Reaction conditions. In a flame dried $4 \mathrm{~mL}$ microwave reaction vial, $\left(\mathrm{NH}_{4}\right)_{2} \mathrm{Fe}\left(\mathrm{SO}_{4}\right)_{2} \cdot 6 \mathrm{H}_{2} \mathrm{O}(1.6$ mg, $0.004 \mathrm{mmol}), 3,4,7,8$-tetramethyl-1,10-phenanthroline (1 mg, $0.004 \mathrm{mmol})$ and $\mathrm{NaOAc}(8.2 \mathrm{mg}, 0.1$ mmol) were mixed together. Then, $0.4 \mathrm{~mL}$ of an aqueous solution of $2 \mathrm{wt} \%$ TPGS-750-M and $0.04 \mathrm{~mL}$ $\mathrm{MeCN}$ were added to the vial followed by sequential addition of olefin ( $0.2 \mathrm{mmol}, 1.0$ equiv) and $\mathrm{Zn}$ dust ( $<10 \mu \mathrm{m}, \geq 98 \%$ from Sigma-Aldrich) (1.2 mmol, 6 equiv). After $5 \mathrm{~min}$ of stirring, the aliphatic bromide (0.6 mmol, 3 equiv) were added and the reaction vial was closed with a rubber septum under air. The reaction mixture was stirred at $25^{\circ} \mathrm{C}$ for $16 \mathrm{~h}$.

For screening experiments, the same procedure as above was followed.

For isolation of the product after $16 \mathrm{~h}$ of stirring, the mixture was extracted with EtOAc $(0.2 \mathrm{~mL} \times 3)$ with the help of centrifuge to phase separation. The combined organic extracts were dried over anhydrous $\mathrm{Na}_{2} \mathrm{SO}_{4}$. Volatiles were removed under reduced pressure to obtain crude product which were further purified by flash chromatography over silica gel using EtOAc/hexanes as eluent. 


\section{Preparation of substrates}

Olefin: 1-(t-butyl)-4-vinylbenzene, 1-bromo-4-vinylbenzene, 1-(trifluoromethyl)-4-vinylbenzene, 1-chloro-2-vinylbenzene, 1-vinylnaphthalene and 2-vinylpyridine are commercially available. Bromides: (3-bromopropoxy)benzene, 4-bromobutyl acetate, 4-bromobut-1-ene, bromocyclohexane and ethyl 3bromopropanoate are commercially available.

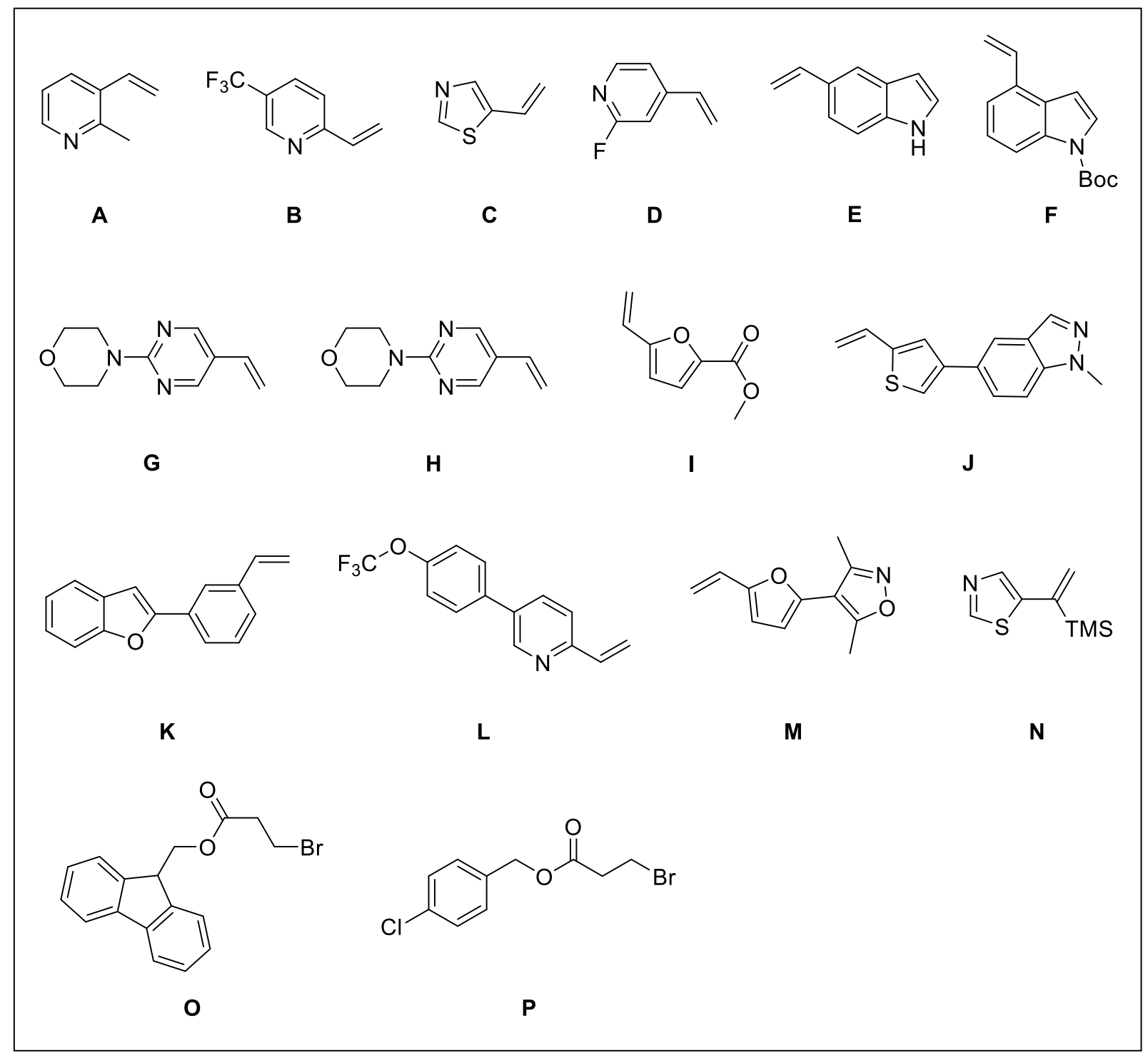

Substrates A - I were synthesized using the general procedure 1 (below) by Suzuki-Miyaura reactions with the corresponding bromides. Substrates $\mathbf{J}-\mathbf{M}$ were synthesised using the general procedure 2 . 
Substrate $\mathbf{N}$ was synthesised by procedure 3. Substrates $\mathbf{O}$ and $\mathbf{P}$ were synthesised by general procedure 4.

\section{General procedure 1}

In an oven-dried $10 \mathrm{~mL}$ round bottom flask containing a PTFE-coated magnetic stir bar, the corresponding bromide ( $2 \mathrm{mmol}, 1$ equiv), vinylboronic acid pinacol ester (2.2 mmol, 1.1 equiv), $\mathrm{Pd}(\mathrm{dtbpf}) \mathrm{Cl}_{2}$ (39.1 mg, $0.06 \mathrm{mmol}, 0.03$ equiv), Et $3 \mathrm{~N}$ (607 mg, $6 \mathrm{mmol}, 3$ equiv) and $4 \mathrm{~mL} 2$ wt \% TPGS$750-\mathrm{M} / \mathrm{H}_{2} \mathrm{O}$ were added. The mixture was stirred under $45^{\circ} \mathrm{C}$ until TLC showed completion. The reaction medium was extracted with EtOAc. The organic layer was separated and dried over anhydrous $\mathrm{Na}_{2} \mathrm{SO}_{4}$. Volatiles were removed under reduced pressure to obtain crude product which was purified by flash chromatography on silica gel with a gradient eluent using hexanes and EtOAc. ${ }^{3}$

\section{General procedure 2}

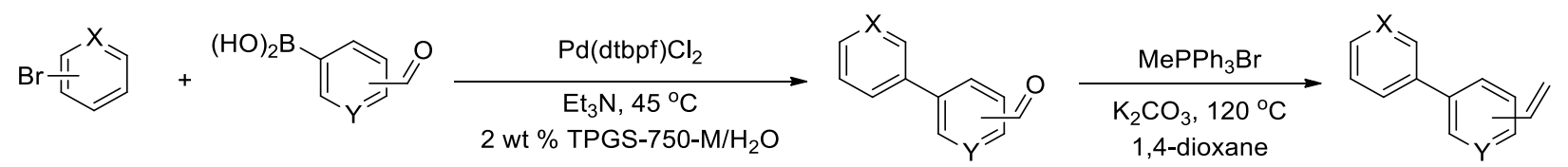

In an oven-dried $10 \mathrm{~mL}$ round bottom flask containing a PTFE-coated magnetic stir bar, corresponding bromide ( $2 \mathrm{mmol}, 1$ equiv), boronic acid (2.2 mmol, 1.1 equiv), $\mathrm{Pd}(\mathrm{dtbpf}) \mathrm{Cl}_{2}(39.1 \mathrm{mg}$, 0.06 mmol, 0.03 equiv), $\mathrm{Et}_{3} \mathrm{~N}$ (607 mg, 6 mmol, 3 equiv) and 2 wt \% TPGS-750-M/ $\mathrm{H}_{2} \mathrm{O}$ were added. The mixture was stirred under $45^{\circ} \mathrm{C}$ until TLC showed completion. The reaction medium was extracted with EtOAc. The organic layer was separated and dried over anhydrous $\mathrm{Na}_{2} \mathrm{SO}_{4}$. Volatiles were removed under reduced pressure to obtain crude product which was purified by flash chromatography on silica gel with a gradient eluent using hexanes and EtOAc. ${ }^{3}$

In an oven-dried $25 \mathrm{~mL}$ round bottom flask containing a PTFE-coated magnetic stir bar, the carbonyl compound (2 mmol, 1 equiv), $\mathrm{Ph}_{3} \mathrm{PMeBr}$ ( $857.3 \mathrm{mg}, 2.4 \mathrm{mmol}, 1.2$ equiv), $\mathrm{K}_{2} \mathrm{CO}_{3}$ (415 mg, 3 mmol, 1.5 equiv) and $5 \mathrm{~mL}$ dioxane were added. The mixture was refluxed at $120^{\circ} \mathrm{C}$ overnight. After completion, the reaction mixture was diluted by EtOAc and then the solution was washed with water three times and then with brine. The organic layer was dried over anhydrous $\mathrm{Na}_{2} \mathrm{SO}_{4}$. Volatiles were removed under reduced pressure to obtain crude product which was purified by flash chromatography on silica gel with a gradient eluent using hexanes and EtOAc. ${ }^{4}$ 


\section{General procedure 3}

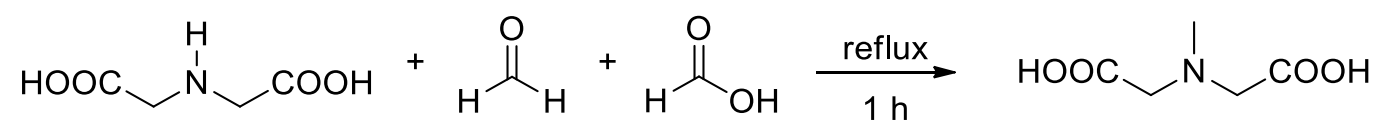

In a 3-neck $50 \mathrm{~mL}$ round bottom flask equipped with a PTFE-coated magnetic stir bar, iminodiacetic acid (6.7 g, $50 \mathrm{mmol}, 1$ equiv) and formic acid (4.6 mg, $100 \mathrm{mmol}, 2$ equiv) were added. A water-cooled Friedrichs condenser vented to the atmosphere was placed on the flask and the mixture was stirred at 90 ${ }^{\circ} \mathrm{C}$ for $30 \mathrm{~min}$. Formalin ( $37 \mathrm{wt} \%$ in $\mathrm{H}_{2} \mathrm{O}, 75 \mathrm{mmol}, 1.5$ equiv) was added drop by drop by syringe over $10 \mathrm{~min}$. Following the addition the mixture was maintained at reflux for an additional $1 \mathrm{~h}$. The solution was cooled to rt and was then transferred to an Erlenmeyer flask. The round bottom flask was washed with water $(4 \times 1.7 \mathrm{~mL}) .50 \mathrm{~mL}$ EtOH was added to the aqueous phase drop by drop. The aqueous phase was left undisturbed overnight, during which time the product crystallized as large colorless needle-like crystals. The crystals were collected via filtration, washed with $\mathrm{EtOH}$ to afford $N$-methyliminodiacetic acid $(6.88 \mathrm{~g}, 93 \%)^{5}$

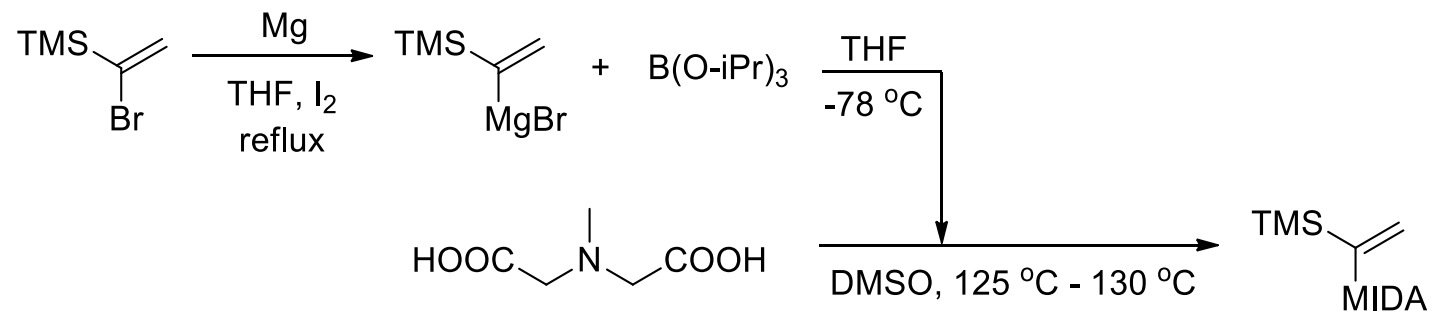

In a 3-neck $50 \mathrm{~mL}$ round bottom flask attached with a water-cooled Friedrichs condenser equipped with a PTFE-coated magnetic stir bar, $\mathrm{Mg}\left(124.8 \mathrm{mg}, 5.2 \mathrm{mmol}, 1.04\right.$ equiv) and $\mathrm{I}_{2}(0.5 \mathrm{mg})$ were added. The flask was sealed with a rubber septum and was assembled and kept under argon. To this flask was added (1-bromovinyl)trimethylsilane ( $895.5 \mathrm{mg}, 5 \mathrm{mmol}, 1$ equiv) and $4 \mathrm{~mL}$ anhydrous THF. The mixture was refluxed until no more $\mathrm{Mg}$ was left in the mixture. To a second round-bottom flask under argon equipped with a Teflon stir bar and septum, B(O-i-Pr) $3(987.4 \mathrm{mg}, 5.25 \mathrm{mmol}, 1.05$ equiv) and $4 \mathrm{~mL}$ anhydrous THF were added and the solution was cooled to $-78{ }^{\circ} \mathrm{C}$. The Grignard reagent was transferred drop by drop to the $\mathrm{B}(\mathrm{O}-i-\mathrm{Pr})_{3}$ solution at $-78{ }^{\circ} \mathrm{C}$ via syringe over $20 \mathrm{~min}$. The mixture was stirred at -78 ${ }^{\circ} \mathrm{C}$ for another $20 \mathrm{~min}$. after which the cooling bath was removed and the solution was allowed to warm to rt. This solution was stirred at $\mathrm{rt}$ for $2 \mathrm{~h}$. 
A dry 3-necked flask, with a stir bar, an addition funnel with a pressure equalizing side arm, along with a jacketed-condenser attached to a 100-mL round bottom flask was assembled and kept under argon as it cooled to rt. Next, $N$-methyliminodiacetic acid $(1.47 \mathrm{~g}, 10 \mathrm{mmol}, 2$ equiv) was added into the $3-$ necked flask followed by DMSO $(6.5 \mathrm{~mL})$ via syringe. The solution was heated to $130^{\circ} \mathrm{C}$ with an oil bath and stirred. Once the reaction medium became homogeneous upon heating, the complex in THF in a second round-bottom flask was added via syringe to the addition funnel. The solution was slowly added to the DMSO solution (over $1 \mathrm{~h}$ ). While the THF was added to the DMSO solution, it was rapidly distilled off during this addition process into a $100 \mathrm{~mL}$ round bottom flask. After the addition was complete, the solution was heated for an additional $30 \mathrm{~min}$ at ca. $120^{\circ} \mathrm{C}$.

Next, the oil bath was set to $80{ }^{\circ} \mathrm{C}$ and the DMSO was slowly distilled off into the round bottom flask under reduced pressure (slowly reducing pressure from 400 torr to $\sim 1$ torr) over the course of $\sim 20$ min. Once nearly all the DMSO was distilled, the oil bath temperature was increased to $100{ }^{\circ} \mathrm{C}$, driving off the remaining DMSO, affording a brown clump, which was purified by flash chromatography on silica gel with a gradient eluent using hexanes and EtOAc, with $\mathrm{KMnO}_{4}$ as stain for monitoring the product by TLC to afford $839 \mathrm{mg}(66 \%)$ 1-trimethylsilanevinylboronic acid MIDA ester as white solid. ${ }^{6}$

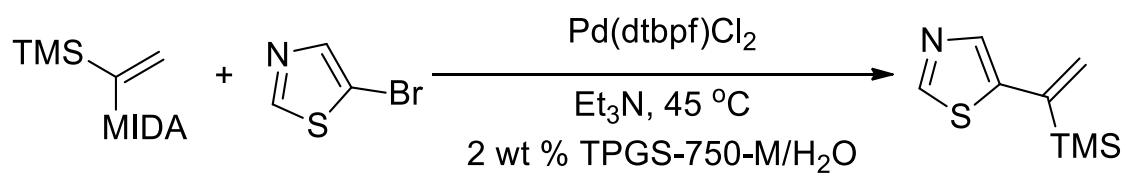

In an oven-dried $10 \mathrm{~mL}$ round bottom flask containing a PTFE-coated magnetic stir bar, 5bromothiazole (162 mg, $1 \mathrm{mmol}$ ), 1-trimethylsilanevinylboronic acid MIDA ester (280.5 mg, $1.1 \mathrm{mmol}$ ), $\mathrm{Pd}(\mathrm{dtbpf}) \mathrm{Cl}_{2}\left(19.6 \mathrm{mg}, 0.03 \mathrm{mmol}\right.$ ), $\mathrm{Et}_{3} \mathrm{~N}$ (304 mg, $3 \mathrm{mmol}$ ) and $2 \mathrm{~mL} 2 \mathrm{wt} \%$ TPGS-750-M/ $\mathrm{H}_{2} \mathrm{O}$ were added. The mixture was stirred under $45^{\circ} \mathrm{C}$ until the TLC showed completion. The reaction medium was extracted with EtOAc. The organic layer was separated and dried over anhydrous $\mathrm{Na}_{2} \mathrm{SO}_{4}$. Volatiles were removed under reduced pressure to obtain crude product which was purified by flash chromatography on silica gel with a gradient eluent using hexanes and EtOAc. ${ }^{3}$

\section{General procedure 4}

3-Bromopropionic acid (3.6 mmol. 1.8 equiv) and the corresponding benzyl alcohol ( $3 \mathrm{mmol}, 1.5$ equiv) were dissolved in $10 \mathrm{~mL}$ dry toluene. A catalytic amount of toluenesulfonic acid monohydrate (0.04 mmol, 0.02 equiv) was added. A Dean-Stark trap and condenser were added and the mixture was 
then heated to $140{ }^{\circ} \mathrm{C}$ overnight, the toluene/water azeotrope mixture was removed 3 times. The toluene was removed by evaporation and the mixture was re-dissolved in EtOAc. The solution was washed with saturated $\mathrm{NaHCO}_{3}$ solution, brine, and then dried over anhydrous $\mathrm{Na}_{2} \mathrm{SO}_{4}$. Volatiles were removed under reduced pressure to obtain crude product which was purified by flash chromatography on silica gel with a gradient eluent using hexanes and EtOAc. ${ }^{7}$

\section{Selectivity on 1-phenylbutadiene}
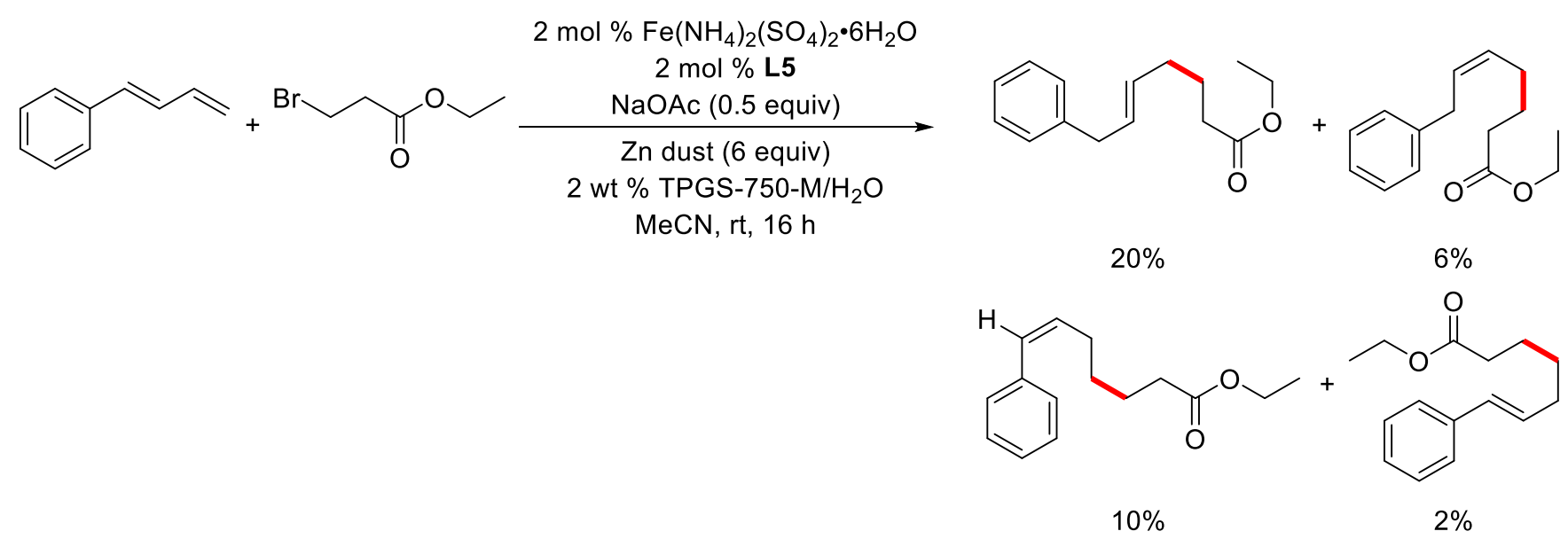

1-Phenylbutadiene (52 mg, $0.4 \mathrm{mmol}$ ), ethyl 3-bromopropanoate (217.2 mg, $1.2 \mathrm{mmol}$ ), $\mathrm{Fe}\left(\mathrm{NH}_{4}\right)_{2}\left(\mathrm{SO}_{4}\right)_{2} \cdot 6 \mathrm{H}_{2} \mathrm{O}(3.2 \mathrm{mg}, 0.008 \mathrm{mmol}), 3,4,7,8$-tetramethyl-1,10-phenanthroline (2 mg, $\left.0.008 \mathrm{mmol}\right)$, Zn dust (156 mg, $2.4 \mathrm{mmol})$ and NaOAc (16.4 mg, $0.2 \mathrm{mmol})$ in $0.8 \mathrm{~mL} 2 \mathrm{wt} \% \mathrm{TPGS}-750-\mathrm{M} / \mathrm{H}_{2} \mathrm{O}$ with $\operatorname{MeCN}(0.08 \mathrm{~mL})$ as co-solvent were reacted at $\mathrm{rt}$ for $16 \mathrm{~h}$. The reaction was monitored by the TLC stained with Seebach's magic TLC stain. After the reaction complete the produced mixture was detected by GCMS and purified by flash chromatography on silica gel yielding $35.3 \mathrm{mg}$ (38\%) of mixture (hexane/EtOAc: 90/10). 


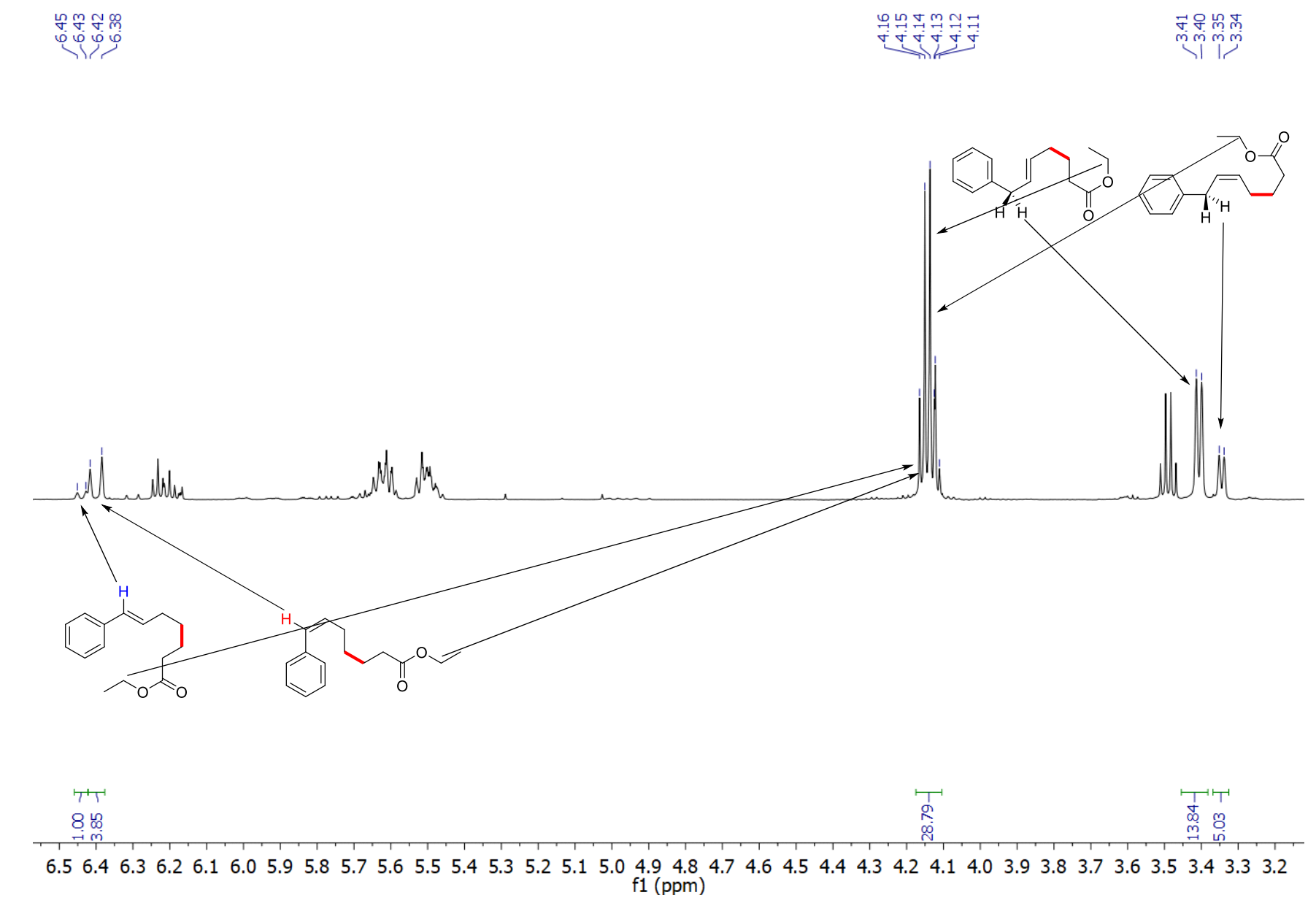

\section{E Factor and Recycle Studies}

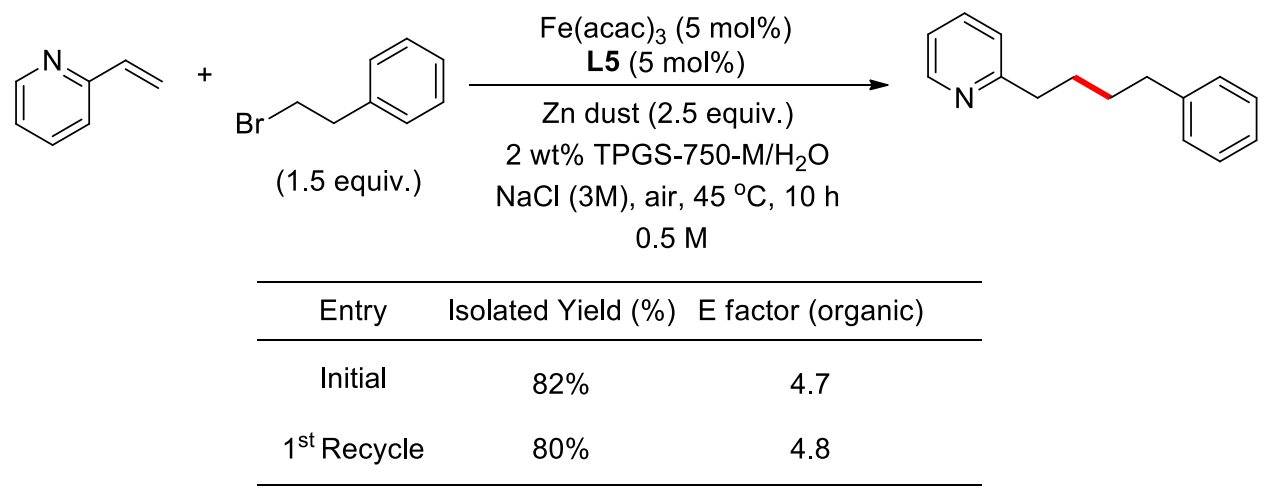

Initial reaction. In a flame dried $4 \mathrm{~mL}$ microwave reaction vial, $\mathrm{Fe}(\mathrm{acac})_{3}(0.025 \mathrm{mmol}, 5 \mathrm{~mol} \%), 3,4,7,8$ tetramethyl-1,10-phenanthroline (0.025 mmol, $5 \mathrm{~mol} \%), \mathrm{NaCl}(70.2 \mathrm{mg}, 1.2 \mathrm{mmol})$ were mixed together. Then, $1.0 \mathrm{~mL}$ aqueous solution $2 \mathrm{wt} \%$ TPGS-750-M was added to the vial followed by sequential addition 
of 2-vinylpyridine ( $0.5 \mathrm{mmol}, 1.0$ equiv) and (2-bromoethyl)benzene ( $0.75 \mathrm{mmol}, 1.5$ equiv). After $5 \mathrm{~min}$ stirring, $\mathrm{Zn}$ dust (1.25 mmol, 2.5 equiv) was added and the reaction vial was closed with a rubber septum under air. The reaction mixture was stirred at $45{ }^{\circ} \mathrm{C}$ for $10 \mathrm{~h}$. The resulting mixture was extracted with EtOAc $(0.15 \mathrm{~mL} \times 3)$. The organic layer was then separated (with the aid of centrifuge, if needed) and dried over anhydrous $\mathrm{Na}_{2} \mathrm{SO}_{4}$, after which the volatiles were removed under reduced pressure and purified by flash chromatography over silica gel with EtOAc/hexanes to obtain 2-(4-phenylbutyl)pyridine. (86.8 $\mathrm{mg}, 0.41 \mathrm{mmol}, 82 \%)$.

$\mathbf{1}^{\text {st }}$ Recycle. In order to remove the $\mathrm{Zn}$, the reaction residue was filtered by fritted glass filter into a new 4 $\mathrm{mL}$ microwave reaction vial. The vial was charged with fresh $\mathrm{Fe}(\mathrm{acac})_{3}(0.025 \mathrm{mmol}, 5 \mathrm{~mol} \%), 3,4,7,8-$ tetramethyl-1,10-phenanthroline ( $0.025 \mathrm{mmol}, 5 \mathrm{~mol} \%)$, 2-vinylpyridine ( $0.5 \mathrm{mmol}, 1.0$ equiv) and 0.5 $\mathrm{mL}$ of an aqueous solution of $2 \mathrm{wt} \%$ TPGS-750-M and the vial was again covered. After $5 \mathrm{~min}$ of stirring, $\mathrm{Zn}$ dust (1.25 mmol, 2.5 equiv) and (2-bromoethyl)benzene ( $0.75 \mathrm{mmol}, 1.5$ equiv) were added and the reaction vial was closed with a rubber septum under air. The reaction mixture was stirred at $45^{\circ} \mathrm{C}$ for 10 h. The resulting mixture was then extracted with EtOAc $(0.15 \mathrm{~mL} \times 3)$. The organic layer was separated (with the aid of centrifuge, if needed) and dried over anhydrous $\mathrm{Na}_{2} \mathrm{SO}_{4}$, after which the volatiles were removed under reduced pressure and purified by flash chromatography over silica gel with EtOAc/hexanes to obtain 2-(4-phenylbutyl)pyridine. (84.6 mg, $0.4 \mathrm{mmol}, 80 \%$ ).

\section{Reaction at the gram scale}

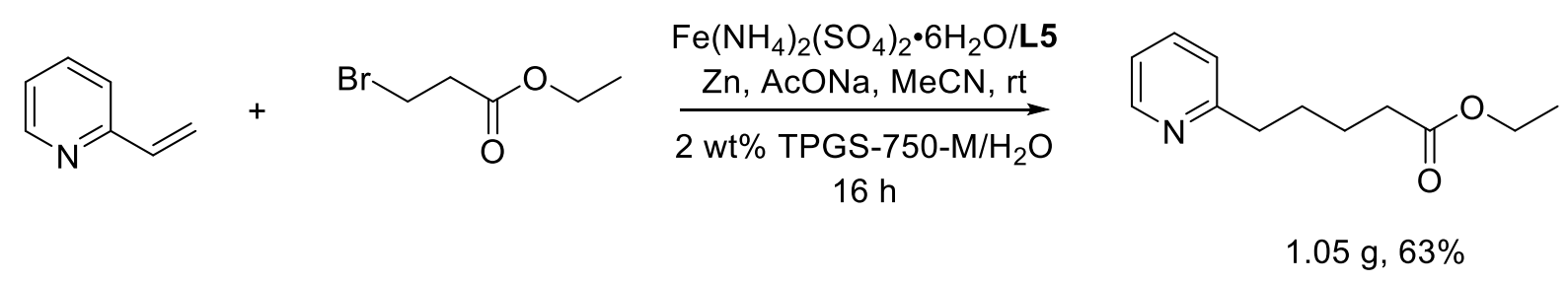

In a flame dried $100 \mathrm{~mL}$ round bottom flask, $\left(\mathrm{NH}_{4}\right)_{2} \mathrm{Fe}\left(\mathrm{SO}_{4}\right)_{2} \cdot 6 \mathrm{H}_{2} \mathrm{O}(62.7 \mathrm{mg}, 0.16 \mathrm{mmol}, 2 \mathrm{~mol}$ $\%$ ), 3,4,7,8-tetramethyl-1,10-phenanthroline (37.8 mg, $0.16 \mathrm{mmol}, 2 \mathrm{~mol} \%)$ and NaOAc (0.328 g, 4 $\mathrm{mmol}$ ) were mixed together. Then, $16 \mathrm{~mL}$ of an aqueous solution of $2 \mathrm{wt} \%$ TPGS-750-M and $2.1 \mathrm{~mL}$ $\mathrm{MeCN}$ were added to the vial followed by sequential addition of 2-vinylpyridine $(0.84 \mathrm{~g}, 8 \mathrm{mmol}, 1.0$ 
equiv.) and Zn dust (3.12 g, $48 \mathrm{mmol}, 6$ equiv). After 5 min of stirring, the ethyl 3-bromopropanoate (4.34 mg, 24 mmol, 3 equiv.) were added and the round bottom flask was closed with a rubber septum under air. The reaction mixture was stirred at $25{ }^{\circ} \mathrm{C}$ for $16 \mathrm{~h}$ after which the mixture was filtered by a fritted glass filter under vacuum. The complex cake was washed by EtOAc. During washing, the cake needed to be stirred in the EtOAc using a spatula. The aqueous medium was further extracted with EtOAc $(10 \mathrm{~mL}$ $\times 5$ ). The combined organic extracts were dried over anhydrous $\mathrm{Na}_{2} \mathrm{SO}_{4}$. Volatiles were removed under reduced pressure to obtain crude product which were further purified by flash chromatography over silica gel using EtOAc/hexanes as eluent yielding $1.05 \mathrm{~g}$ (63\%) ethyl 5-(pyridin-2-yl)pentanoate.

\section{Synthesis of the precursor to a SphK inhibitor}

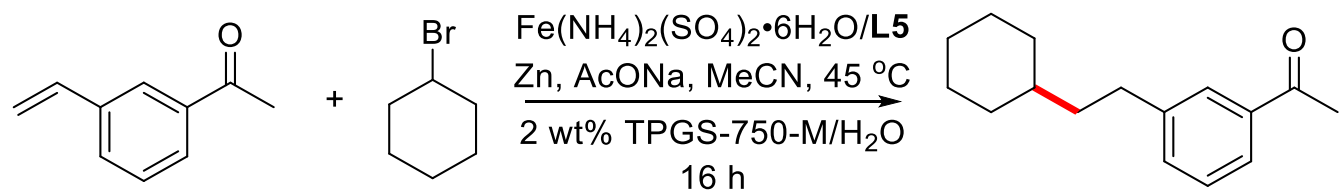

$5166 \%$

1-(3-Vinylphenyl)ethanone (58.5 mg, $0.4 \mathrm{mmol})$, bromocyclohexane (195.7 mg, $1.2 \mathrm{mmol})$, $\mathrm{Fe}\left(\mathrm{NH}_{4}\right)_{2}\left(\mathrm{SO}_{4}\right)_{2} \cdot 6 \mathrm{H}_{2} \mathrm{O}(3.2 \mathrm{mg}, 0.008 \mathrm{mmol}), 3,4,7,8$-tetramethyl-1,10-phenanthroline (2 $\left.\mathrm{mg}, 0.008 \mathrm{mmol}\right)$, Zn dust (156 mg, $2.4 \mathrm{mmol})$ and NaOAc (16.4 mg, $0.2 \mathrm{mmol})$, in $0.8 \mathrm{~mL} 2$ wt \% TPGS-750-M/ $\mathrm{H}_{2} \mathrm{O}$ with $\mathrm{MeCN}(0.08 \mathrm{~mL})$ as co-solvent were reacted at $45{ }^{\circ} \mathrm{C}$ for $16 \mathrm{~h}$ yielding $60.7 \mathrm{mg}(66 \%)$ of 1-(3-(2cyclohexylethyl)phenyl)ethanone as a slight yellow oil (hexane/EtOAc: 90/10).

${ }^{1} \mathrm{H}$ NMR (500 MHz, $\left.\mathrm{CDCl}_{3}\right) \delta 7.81-7.72(\mathrm{~m}, 2 \mathrm{H}), 7.39-7.33(\mathrm{~m}, 2 \mathrm{H}), 2.69-2.63(\mathrm{~m}, 2 \mathrm{H}), 2.59$ (s, 3H), $1.78-1.62(\mathrm{~m}, 5 \mathrm{H}), 1.55-1.48(\mathrm{~m}, 2 \mathrm{H}), 1.27-1.14(\mathrm{~m}, 4 \mathrm{H}), 1.00-0.89(\mathrm{~m}, 2 \mathrm{H})$.

${ }^{13} \mathrm{C}$ NMR $\left(126 \mathrm{MHz}, \mathrm{CDCl}_{3}\right) \delta 198.49,143.86,137.31,133.31,128.54,128.13,125.91,39.40$, $37.45,33.38,33.25,26.77,26.75,26.41$.

HRMS(EI): Calcd. for $\mathrm{C}_{16} \mathrm{H}_{22} \mathrm{O}[\mathrm{M}]^{+}$230.1671. Found: 230.1668 . 


\section{Mechanistic studies}

a) Control experiments

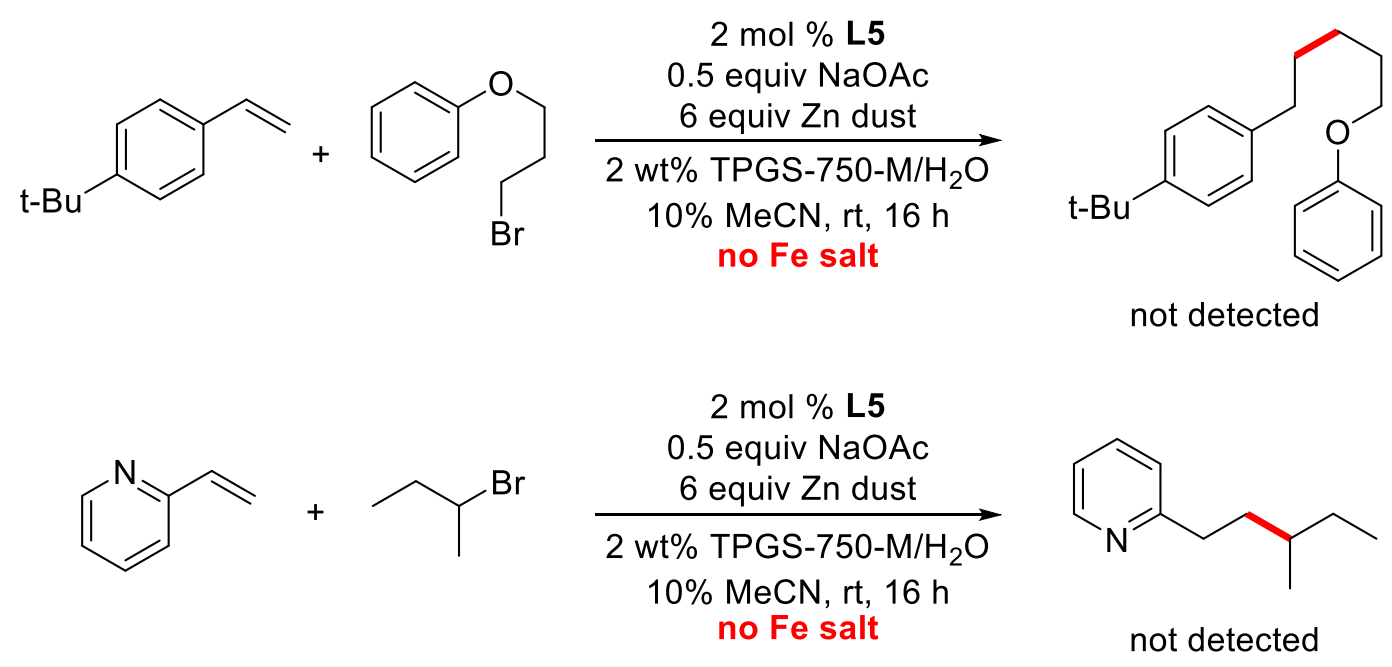

1-(t-Butyl)-4-vinylbenzene (32 mg, $0.2 \mathrm{mmol})$, (3-bromopropoxy)benzene (129.1 mg, $0.6 \mathrm{mmol})$, 3,4,7,8-tetramethyl-1,10-phenanthroline (1 mg, $0.004 \mathrm{mmol})$, Zn dust (78 mg, $1.2 \mathrm{mmol})$, NaOAc (8.2 $\mathrm{mg}, 0.1 \mathrm{mmol})$ in $0.4 \mathrm{~mL} 2 \mathrm{wt} \%$ TPGS-750-M/ $\mathrm{H}_{2} \mathrm{O}$ with $\mathrm{MeCN}(0.04 \mathrm{~mL})$ as co-solvent were reacted at $\mathrm{rt}$ for $16 \mathrm{~h}$. After $16 \mathrm{~h}$, EtOAc was added and the mixture stirred for $2 \mathrm{~min}$ at rt. Stirring was then stopped and the organic layer was decanted via pipette. The organic layer was passed through a very small silica plug. Yields were determined by GC using naphthalene as internal standard.

2-Vinylpyridine (21 mg, $0.2 \mathrm{mmol}$ ), 2-bromobutane (82.2 mg, $0.6 \mathrm{mmol}), 3,4,7,8$-tetramethyl1,10-phenanthroline (1 mg, $0.004 \mathrm{mmol}), \mathrm{Zn}$ dust (78 mg, $1.2 \mathrm{mmol})$ and NaOAc (8.2 mg, $0.1 \mathrm{mmol})$, in $0.4 \mathrm{~mL} 2 \mathrm{wt} \%$ TPGS-750-M/ $\mathrm{H}_{2} \mathrm{O}$ with $\mathrm{MeCN}(0.04 \mathrm{~mL})$ as co-solvent were reacted at $\mathrm{rt}$ for $16 \mathrm{~h}$. Stirring was then stopped and the organic layer was decanted via pipette. The organic layer was passed through a very small silica plug. Yields were determined by GC using naphthalene as internal standard.

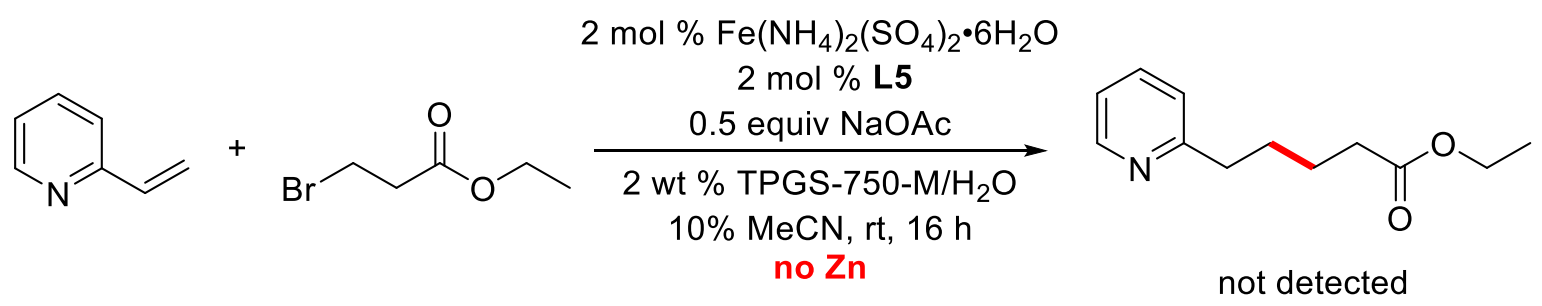


2-Vinylpyridine (21 mg, $0.2 \mathrm{mmol}$ ), ethyl 3-bromopropanoate (108.6 mg, $0.6 \mathrm{mmol}$ ), $\mathrm{Fe}\left(\mathrm{NH}_{4}\right)_{2}\left(\mathrm{SO}_{4}\right)_{2} \cdot 6 \mathrm{H}_{2} \mathrm{O}(1.6 \mathrm{mg}, 0.004 \mathrm{mmol}), 3,4,7,8$-tetramethyl-1,10-phenanthroline (1 mg, $\left.0.004 \mathrm{mmol}\right)$ and $\mathrm{NaOAc}(8.2 \mathrm{mg}, 0.1 \mathrm{mmol})$, in $0.4 \mathrm{~mL} 2 \mathrm{wt} \% \mathrm{TPGS}-750-\mathrm{M} / \mathrm{H}_{2} \mathrm{O}$ with $\mathrm{MeCN}(0.04 \mathrm{~mL})$ as co-solvent were reacted at $\mathrm{rt}$ for $16 \mathrm{~h}$. Stirring was then stopped and the organic layer was decanted via pipette. The organic layer was passed through a very small silica plug. Yields were determined by GC using naphthalene as internal standard.

\section{b) $\mathrm{H} / \mathrm{D}$ transfer experiment}

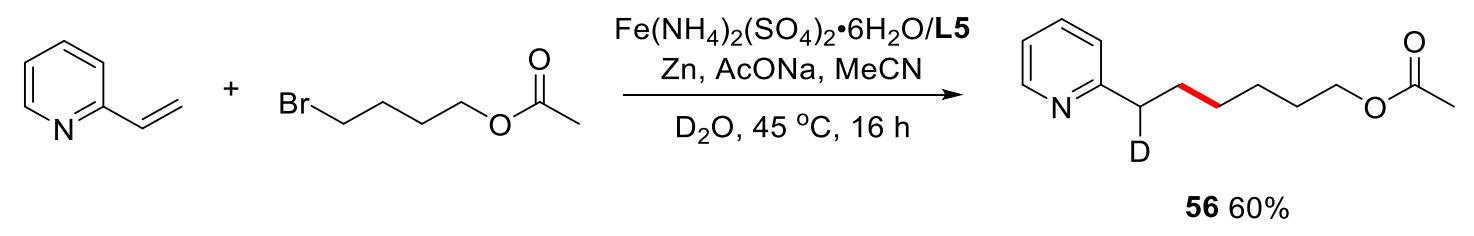

To investigate the proposed mechanism of this reaction, an experiment was run using proton NMR to monitor product(s) formation. In the first H/D experiment, 2-vinylpyridine (21 mg, $0.2 \mathrm{mmol})$, 4bromobutyl acetate (117 mg, $0.6 \mathrm{mmol}), \mathrm{Fe}\left(\mathrm{NH}_{4}\right)_{2}\left(\mathrm{SO}_{4}\right)_{2} \cdot 6 \mathrm{H}_{2} \mathrm{O}$ (1.6 mg $\left.0.004 \mathrm{mmol}\right)$, 3,4,7,8tetramethyl-1,10-phenanthroline (1 mg, $0.004 \mathrm{mmol}), \mathrm{NaOAc}(8.2 \mathrm{mg}, 0.1 \mathrm{mmol}), \mathrm{MeCN}(0.04 \mathrm{~mL})$ and $\mathrm{Zn}$ dust $(78 \mathrm{mg}, 1.2 \mathrm{mmol})$ were placed in the $0.4 \mathrm{~mL}$ of $\mathrm{D}_{2} \mathrm{O}$ and the reaction conducted at $45^{\circ} \mathrm{C}$ for 16 h yielding $26.8 \mathrm{mg}(60 \%)$ of 6-(pyridin-2-yl)hexyl-6-d acetate as a faint yellow oil (hexane/EtOAc: 80/20).

${ }^{1} \mathrm{H} \mathrm{NMR}\left(500 \mathrm{MHz}, \mathrm{CDCl}_{3}\right) \delta 8.50(\mathrm{~d}, J=4.2 \mathrm{~Hz}, 1 \mathrm{H}), 7.56(\mathrm{td}, J=7.7,1.8 \mathrm{~Hz}, 1 \mathrm{H}), 7.14-7.04$ $(\mathrm{m}, 2 \mathrm{H}), 4.02(\mathrm{t}, J=6.7 \mathrm{~Hz}, 2 \mathrm{H}), 2.75(\mathrm{t}, J=7.8 \mathrm{~Hz}, 1 \mathrm{H}), 2.01(\mathrm{~s}, 3 \mathrm{H}), 1.75-1.68(\mathrm{~m}, 2 \mathrm{H}), 1.60(\mathrm{p}, J=$ $6.9 \mathrm{~Hz}, 2 \mathrm{H}), 1.37$ (p, $J=3.6 \mathrm{~Hz}, 4 \mathrm{H})$.

${ }^{13} \mathrm{C} \mathrm{NMR}\left(126 \mathrm{MHz}, \mathrm{CDCl}_{3}\right) \delta 171.25,162.29,149.27,136.32,122.75,120.99,64.60,37.99(\mathrm{~m})$, $29.71,29.00,28.58,25.86,21.05$.

${ }^{2} \mathrm{H}$ NMR $\left(61 \mathrm{MHz}, \mathrm{CHCl}_{3}\right) \delta 2.76$.

HRMS(ESI): Calcd. for $\mathrm{C}_{13} \mathrm{H}_{19} \mathrm{DNO}_{2}[\mathrm{M}+\mathrm{H}]^{+}$223.1557. Found: 223.1552 .

The product was analyzed by ${ }^{1} \mathrm{H}$ NMR and compared with the ${ }^{1} \mathrm{H}$ NMR of the product from 2 wt $\%$ TPGS-750-M/ $\mathrm{H}_{2} \mathrm{O}$. This experiment indicated that the proton/deuteron in the product comes from the medium, in this case, $\mathrm{D}_{2} \mathrm{O}$. 

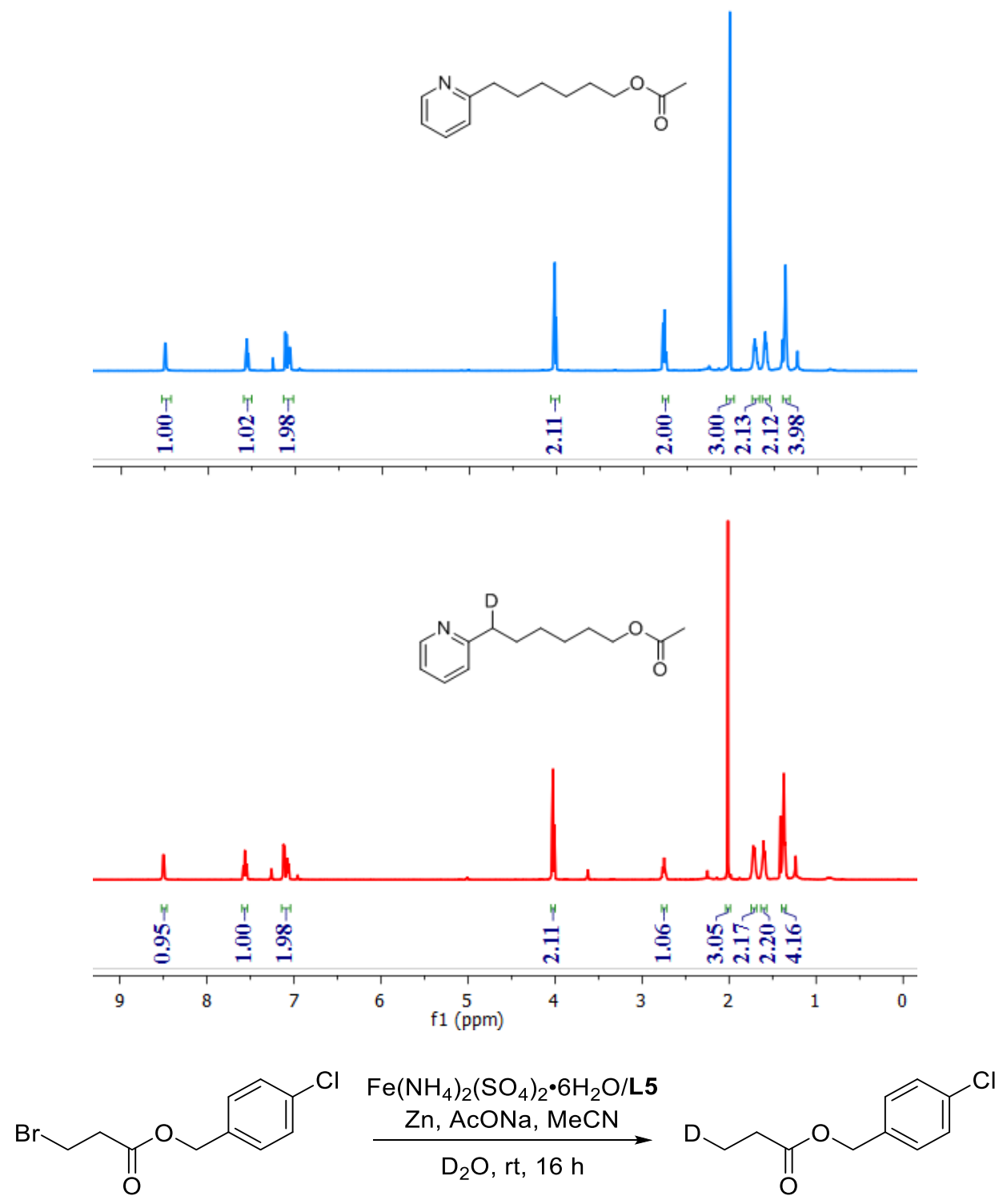

In addition, the main by-product from debromination was analyzed by a similar experiment. Hence, 4-chlorobenzyl 3-bromopropanoate (166.5 mg, $0.6 \mathrm{mmol}), \mathrm{Fe}\left(\mathrm{NH}_{4}\right)_{2}\left(\mathrm{SO}_{4}\right)_{2} \cdot 6 \mathrm{H}_{2} \mathrm{O}(1.6 \mathrm{mg} 0.004 \mathrm{mmol})$, 3,4,7,8-tetramethyl-1,10-phenanthroline (1 mg, $0.004 \mathrm{mmol}), \mathrm{NaOAc}$ (8.2 mg, $0.1 \mathrm{mmol}), \mathrm{MeCN}$ (0.04 $\mathrm{mL}$ ) and $\mathrm{Zn}$ dust (78 mg, $1.2 \mathrm{mmol}$ ) were placed in reaction vessel containing $0.4 \mathrm{~mL} \mathrm{D}_{2} \mathrm{O}$ and the reaction was run for $16 \mathrm{~h}$. The product was analyzed by ${ }^{1} \mathrm{H}$ NMR and compared with the ${ }^{1} \mathrm{H}$ NMR derived from 4chlorobenzyl 3-bromopropanoate. The experiment indicated the proton of the by-product also comes from $\mathrm{D}_{2} \mathrm{O}$. 

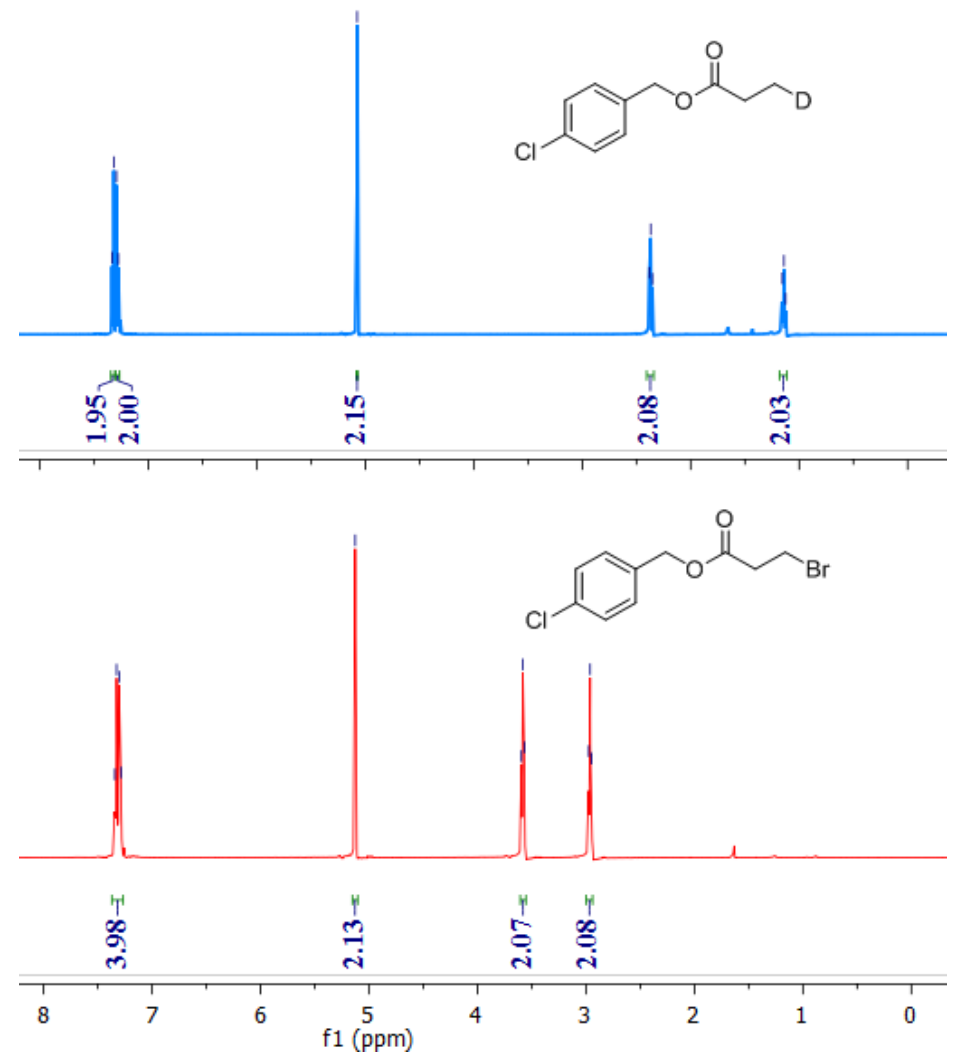

\section{c) Radical clock experiments}

\section{Substrate synthesis}

\section{(1-Cyclopropylvinyl)benzene}<smiles>C=C(c1ccccc1)C1CC1</smiles>

In a $250 \mathrm{~mL}$ round bottom flask methyltriphenylphosphonium bromide (7.2 g, $20 \mathrm{mmol})$ was dissolved in anhydrous THF $(60 \mathrm{~mL})$ and the mixture was cooled to $-78{ }^{\circ} \mathrm{C}$ under an argon atmosphere. $n$-BuLi (8.0 mL, $2.5 \mathrm{M}, 20 \mathrm{mmol})$ was added dropwise within $45 \mathrm{~min}$, after which the cooling was removed. To this mixture cyclopropyl phenyl ketone $(1.46 \mathrm{~g}, 10.0 \mathrm{mmol})$ dissolved in anhydrous THF $(6 \mathrm{~mL}) \mathrm{was}$ added dropwise and the reaction was left to stir for $1 \mathrm{~h}$. The reaction was monitored by TLC. Brine (30 $\mathrm{mL})$ was added and the phases were separated. The aqueous phase was extracted with pentane $(3 \times 20$ $\mathrm{mL}$ ), and the combined organic phases were dried over anhydrous $\mathrm{MgSO}_{4}$, filtered and the crude mixture 
was further purified by flash chromatography over silica gel using pure pentane as eluent yielding $1.43 \mathrm{~g}$ (99\%) (1-cyclopropylvinyl)benzene as a colorless oil.

Spectral data matched that reported in the literature. ${ }^{8}$

${ }^{1} \mathrm{H}$ NMR $\left(500 \mathrm{MHz}, \mathrm{CDCl}_{3}\right) \delta 7.66$ - $7.53(\mathrm{~m}, 2 \mathrm{H}), 7.40$ - $7.31(\mathrm{~m}, 2 \mathrm{H}), 7.31-7.26(\mathrm{~m}, 1 \mathrm{H}), 5.29$ (s, 1H), $4.95(\mathrm{~s}, 1 \mathrm{H}), 1.67$ (dddd, $J=13.6,8.2,5.4,1.1 \mathrm{~Hz}, 1 \mathrm{H}), 0.87-0.79(\mathrm{~m}, 2 \mathrm{H}), 0.64-0.55(\mathrm{~m}, 2 \mathrm{H})$.

${ }^{13} \mathrm{C} \mathrm{NMR}\left(126 \mathrm{MHz} \mathrm{CDCl}_{3}\right) \delta 149.52,141.79,128.28,127.58,126.26,109.14,15.78,6.82$. $m / z: 144\left[\mathrm{M}^{+}\right]$

\section{Radical clock reaction}

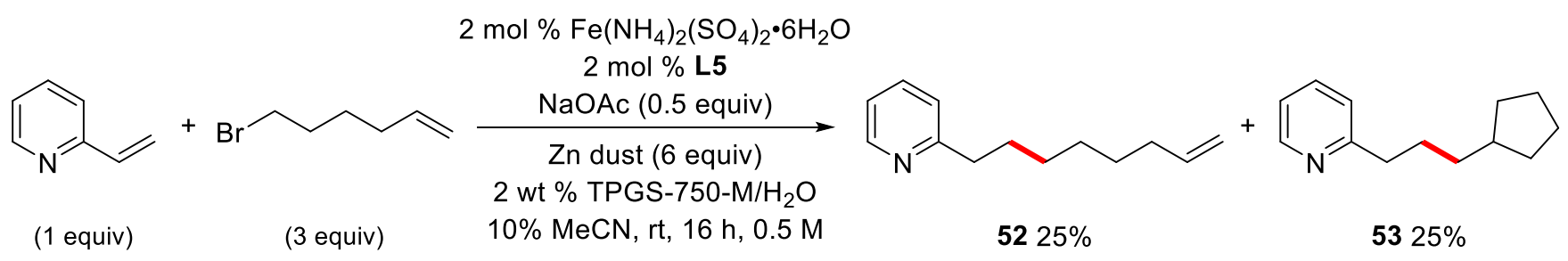

2-Vinylpyridine (42 mg, $0.4 \mathrm{mmol}), \quad$ 6-bromohex-1-ene $(195.7 \mathrm{mg}, \quad 1.2 \mathrm{mmol})$, $\mathrm{Fe}\left(\mathrm{NH}_{4}\right)_{2}\left(\mathrm{SO}_{4}\right)_{2} \cdot 6 \mathrm{H}_{2} \mathrm{O}(3.2 \mathrm{mg}, 0.008 \mathrm{mmol}), 3,4,7,8$-tetramethyl-1,10-phenanthroline (2 mg, $\left.0.008 \mathrm{mmol}\right)$, Zn dust (156 mg, $2.4 \mathrm{mmol})$ and NaOAc (16.4 mg, $0.2 \mathrm{mmol})$ were added to $0.8 \mathrm{~mL} 2$ wt \% TPGS-750$\mathrm{M} / \mathrm{H}_{2} \mathrm{O}$ with $\mathrm{MeCN}(0.08 \mathrm{~mL})$ as co-solvent, and the mixture was vigorously stirred at $\mathrm{rt}$ for $16 \mathrm{~h}$ yielding $37.8 \mathrm{mg}(50 \%)$ of a mixture containing 2-(oct-7-en-1-yl)pyridine (25\%) and 2-(3cyclopentylpropyl)pyridine (25\%) as a faint yellow oil (hexane/EtOAc: $85 / 15)$.

${ }^{1} \mathrm{H}$ NMR $\left(500 \mathrm{MHz}, \mathrm{CDCl}_{3}\right) \delta 8.57-8.43(\mathrm{~m}, 2 \mathrm{H}), 7.57-7.50(\mathrm{~m}, 2 \mathrm{H}), 7.09(\mathrm{~d}, J=7.7 \mathrm{~Hz}, 2 \mathrm{H})$, $7.07-7.01(\mathrm{~m}, 2 \mathrm{H}), 5.76(\mathrm{ddt}, J=16.9,10.2,6.7 \mathrm{~Hz}, 1 \mathrm{H}), 4.98-4.92(\mathrm{~m}, 1 \mathrm{H}), 4.89(\mathrm{~d}, J=10.2 \mathrm{~Hz}, 1 \mathrm{H})$, $2.74(\mathrm{t}, J=7.8 \mathrm{~Hz}, 4 \mathrm{H}), 2.00(\mathrm{q}, J=6.8,6.4 \mathrm{~Hz}, 2 \mathrm{H}), 1.80-1.62(\mathrm{~m}, 7 \mathrm{H}), 1.57-1.43(\mathrm{~m}, 3 \mathrm{H}), 1.39-1.27$ $(\mathrm{m}, 9 \mathrm{H}), 1.12-0.97(\mathrm{~m}, 2 \mathrm{H})$.

${ }^{13} \mathrm{C} \mathrm{NMR}\left(126 \mathrm{MHz}, \mathrm{CDCl}_{3}\right) \delta 162.60,162.51,149.23,139.13,136.23,122.71,120.88,120.86$, $114.23,40.12,38.79,38.48,36.01,33.81,32.74,29.91,29.28,29.21,29.01,28.86,25.23$.

HRMS(EI): Calcd. for $\mathrm{C}_{13} \mathrm{H}_{19} \mathrm{~N}[\mathrm{M}]^{+}$189.1517. Found: 189.1513. 


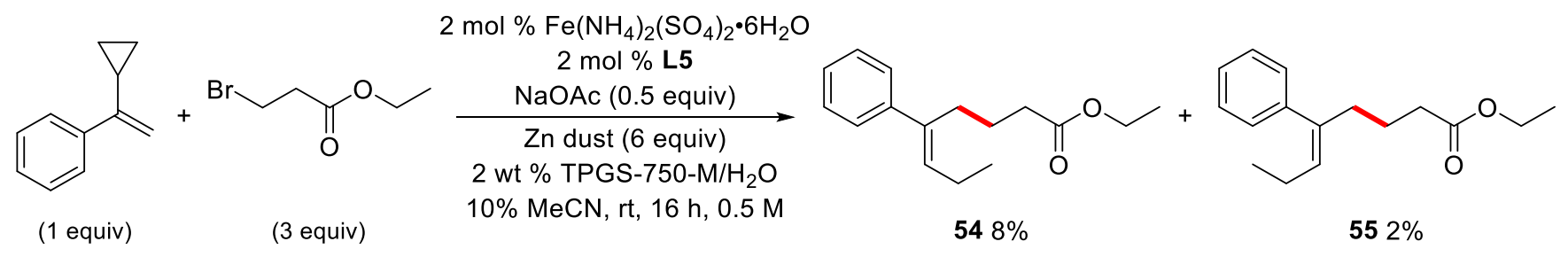

Cyclopropylvinyl)benzene (576.8 mg, $4 \mathrm{mmol})$, ethyl 3-bromopropanoate (2.17 g, $12 \mathrm{mmol})$, $\mathrm{Fe}\left(\mathrm{NH}_{4}\right)_{2}\left(\mathrm{SO}_{4}\right)_{2} \cdot 6 \mathrm{H}_{2} \mathrm{O}$ (32 mg, $0.08 \mathrm{mmol}$ ), 3,4,7,8-tetramethyl-1,10-phenanthroline (20 mg, $0.08 \mathrm{mmol}$ ), Zn dust (1.56 g, $24 \mathrm{mmol}$ ) and $\mathrm{NaOAc}\left(164 \mathrm{mg}, 2 \mathrm{mmol}\right.$ ), in $8 \mathrm{~mL} 2 \mathrm{wt} \%$ TPGS-750-M/ $\mathrm{H}_{2} \mathrm{O}$ with $\mathrm{MeCN}$ $(0.8 \mathrm{~mL})$ as co-solvent were reacted at $\mathrm{rt}$ for $16 \mathrm{~h}$ yielding $98.5 \mathrm{mg}(10 \%)$ of a mixture with ethyl (E)-5phenyloct-5-enoate (8\%) and ethyl (Z)-5-phenyloct-5-enoate (2\%) as a colorless oil (hexane/Et $2 \mathrm{O}: 90 / 10)$.

${ }^{1} \mathrm{H}$ NMR $\left(500 \mathrm{MHz}, \mathrm{CDCl}_{3}\right) \delta(\mathrm{E}) 7.36-7.28(\mathrm{~m}, 4 \mathrm{H}), 7.25-7.20(\mathrm{~m}, 1 \mathrm{H}), 5.70(\mathrm{t}, J=7.2 \mathrm{~Hz}$, $1 \mathrm{H}), 4.12(\mathrm{q}, J=7.1 \mathrm{~Hz}, 2 \mathrm{H}), 2.58-2.53(\mathrm{~m}, 2 \mathrm{H}), 2.28(\mathrm{t}, J=7.4 \mathrm{~Hz}, 2 \mathrm{H}), 2.21(\mathrm{p}, J=7.5 \mathrm{~Hz}, 2 \mathrm{H}), 1.72$ - $1.64(\mathrm{~m}, 2 \mathrm{H}), 1.25$ (t, $J=7.1 \mathrm{~Hz}, 3 \mathrm{H}), 1.07$ (t, $J=7.5 \mathrm{~Hz}, 3 \mathrm{H})$. (Z) $7.36-7.28(\mathrm{~m}, 2 \mathrm{H}), 7.25-7.20$ (m, 1H), $7.16-7.12(\mathrm{~m}, 2 \mathrm{H}), 5.46(\mathrm{t}, J=7.3 \mathrm{~Hz}, 1 \mathrm{H}), 4.11(\mathrm{q}, J=7.1 \mathrm{~Hz}, 2 \mathrm{H}), 2.37(\mathrm{t}, J=7.4 \mathrm{~Hz}, 2 \mathrm{H}), 2.31-$ $2.26(\mathrm{~m}, 2 \mathrm{H}), 1.95$ (p, $J=7.4 \mathrm{~Hz}, 2 \mathrm{H}), 1.72-1.64(\mathrm{~m}, 2 \mathrm{H}), 1.24$ (t, $J=7.1 \mathrm{~Hz}, 3 \mathrm{H}), 0.93$ (t, $J=7.5 \mathrm{~Hz}$, $3 \mathrm{H})$.

${ }^{13} \mathrm{C}$ NMR (126 MHz, $\left.\mathrm{CDCl}_{3}\right) \delta$ (E) 173.65, 142.96, 138.52, 131.75, 128.34, 126.69, 126.41, 60.34, 33.87, 28.91, 23.90, 21.98, 14.52, 14.36. (Z) 173.78, 140.98, 139.29, 130.07, 128.50, 128.15, 126.59, 60.28, $38.53,33.72,23.50,22.36,14.78,14.36$.

HRMS(ESI): Calcd. for $\mathrm{C}_{16} \mathrm{H}_{22} \mathrm{O}_{2} \mathrm{Na}[\mathrm{M}+\mathrm{Na}]^{+}$269.1518. Found: 269.1522.

\section{d) Radical intermediate trapping test}



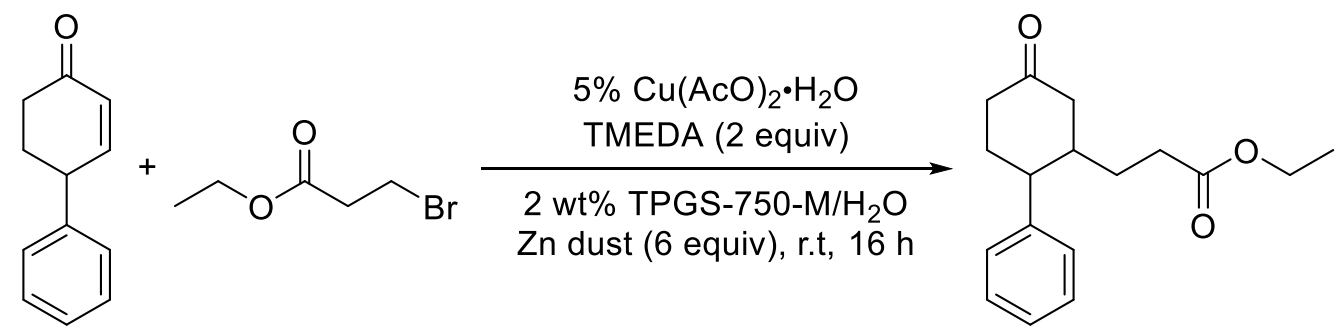

GC-MS yield: $3 \%$
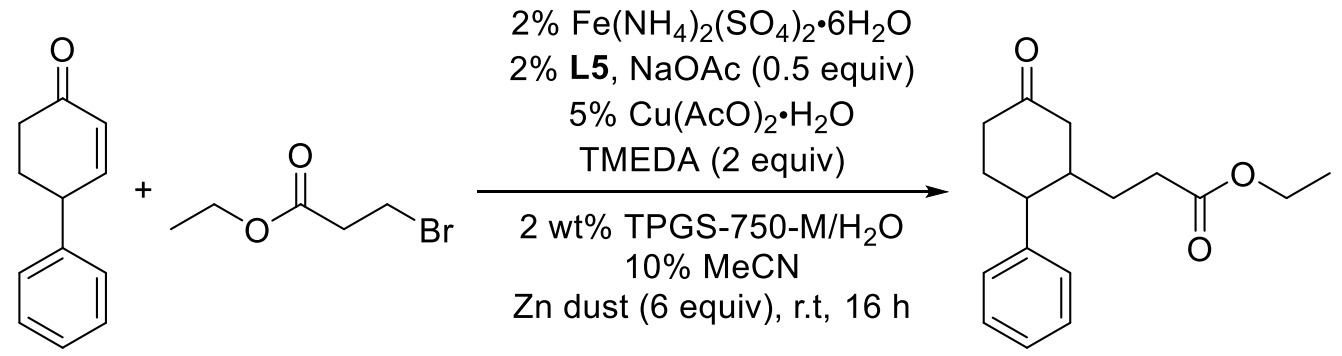

GC-MS yield: $13 \%$

No iron salts. Following the procedure reported in the literature, ${ }^{9}$ to $4 \mathrm{~mL}$ microwave vial (ovendried and under Ar) containing a Teflon ${ }^{\circledR}$ stir bar was charged with zinc powder $(78 \mathrm{mg}, 1.2 \mathrm{mmol})$, $\mathrm{Cu}(\mathrm{OAc})_{2} \cdot \mathrm{H}_{2} \mathrm{O}(2.0 \mathrm{mg}, 0.01 \mathrm{mmol}, 5 \mathrm{~mol} \%)$, 4-phenyl-2-cyclohexenone (34.4 mg, $\left.0.2 \mathrm{mmol}\right)$, and $N, N, N^{\prime}, N^{\prime}$ '-tetramethylethylene-diamine (TMEDA, $46.5 \mathrm{mg}, 0.4 \mathrm{mmol}$ ). The vial was capped with a rubber septum and placed under an argon atmosphere, and $0.4 \mathrm{~mL}$ of a $2 \mathrm{wt} \%$ TPGS-750-M/ $\mathrm{H}_{2} \mathrm{O}$ was added via syringe followed by the addition of ethyl 3-bromopropanoate $(108.6 \mathrm{mg}, 0.6 \mathrm{mmol})$. The resulting mixture was stirred vigorously at rt. After $16 \mathrm{~h}$, EtOAc was added and the mixture was stirred for $2 \mathrm{~min}$ at rt. Stirring was then stopped and the organic layer was decanted via pipette. The organic layer was passed through a very small silica plug. Yields were determined by GC using naphthalene as internal standard.

With iron salts. To a flame dried $4 \mathrm{~mL}$ microwave reaction vial, $\left(\mathrm{NH}_{4}\right)_{2} \mathrm{Fe}\left(\mathrm{SO}_{4}\right)_{2} \cdot 6 \mathrm{H}_{2} \mathrm{O}(1.6 \mathrm{mg}$, $0.004 \mathrm{mmol}), 3,4,7,8$-tetramethyl-1,10-phenanthroline (1 mg, $0.004 \mathrm{mmol})$ and $\mathrm{NaOAc}(8.2 \mathrm{mg}, 0.1$ $\mathrm{mmol}$ ) were mixed together. Then, $0.4 \mathrm{~mL}$ of an aqueous solution of $2 \mathrm{wt} \%$ TPGS-750- $-\mathrm{M}^{2} \mathrm{H}_{2} \mathrm{O}$ and 0.04 $\mathrm{mL} \mathrm{MeCN}$ were added to the vial followed by sequential addition of 4-phenyl-2-cyclohexenone (34.4 $\mathrm{mg}$, $0.2 \mathrm{mmol}$ ) and $\mathrm{Zn}$ dust ( $<10 \mu \mathrm{m}, \geq 98 \%$ from Sigma-Aldrich; $78 \mathrm{mg}, 1.2 \mathrm{mmol}, 6$ equiv). After $5 \mathrm{~min}$ of stirring, $\mathrm{Cu}(\mathrm{OAc})_{2} \cdot \mathrm{H}_{2} \mathrm{O}(2.0 \mathrm{mg}, 0.01 \mathrm{mmol}, 5 \mathrm{~mol} \%)$ and TMEDA (46.5 mg, $\left.0.4 \mathrm{mmol}\right)$ were added to the mixture. The vial was capped with a rubber septum and placed under an argon atmosphere. ethyl 3bromopropanoate ( $108.6 \mathrm{mg}, 0.6 \mathrm{mmol}, 3$ equiv) was added via syringe. The reaction mixture was stirred 
at rt. After $16 \mathrm{~h}$, EtOAc was added and the mixture was stirred for $2 \mathrm{~min}$ at rt. Stirring was then stopped and the organic layer was decanted via pipette. The organic layer was passed through a very small silica plug. Yields were determined by GC using naphthalene as internal standard.

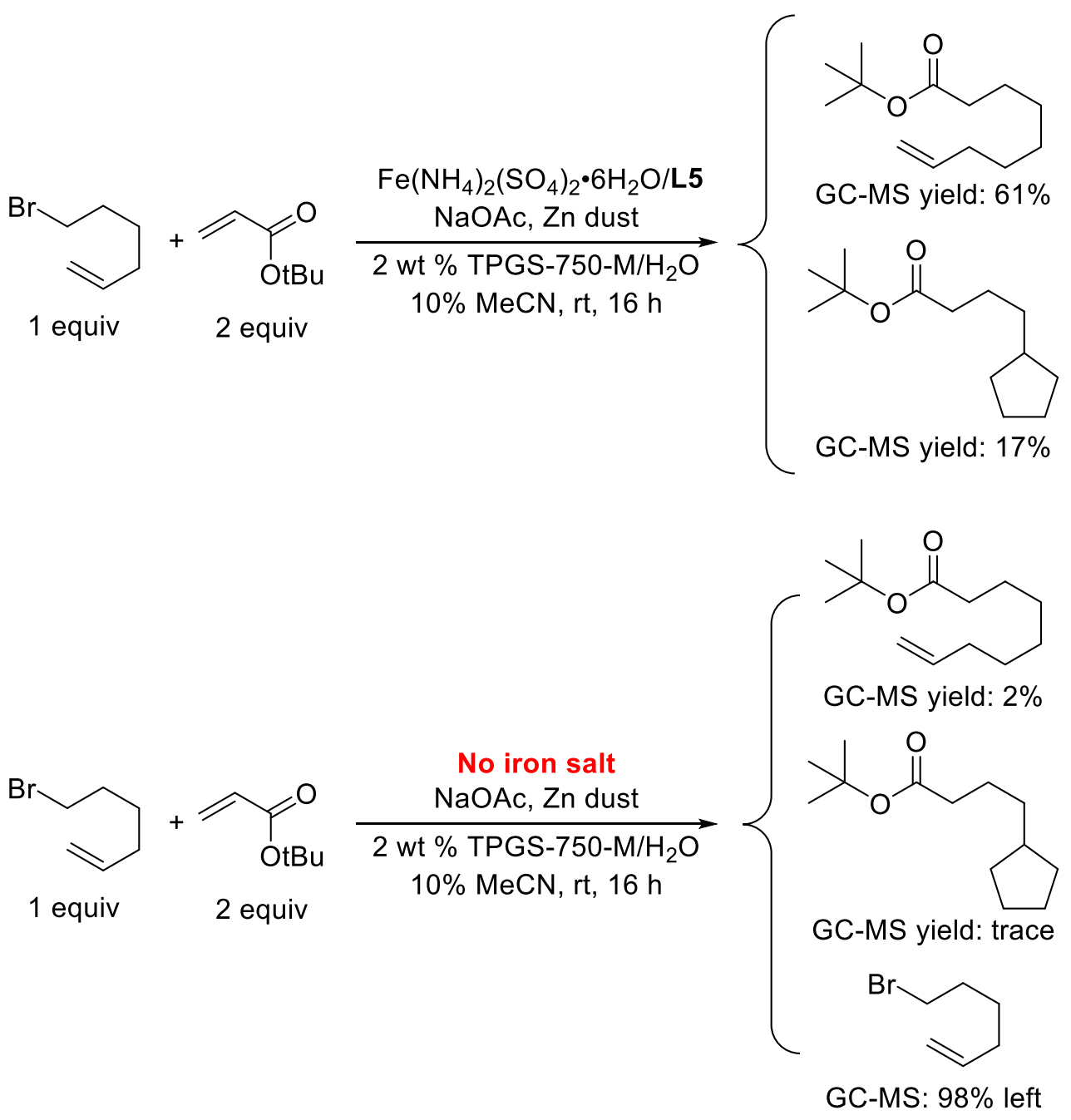

With iron salts. To a flame dried $4 \mathrm{~mL}$ microwave reaction vial, 6-bromohex-1-ene (195.7 mg, 1.2 mmol), tert-butyl acrylate (307.7 mg, $2.4 \mathrm{mmol}), \mathrm{Fe}\left(\mathrm{NH}_{4}\right)_{2}\left(\mathrm{SO}_{4}\right)_{2} \cdot 6 \mathrm{H}_{2} \mathrm{O}(3.2 \mathrm{mg}, 0.008 \mathrm{mmol}), 3,4,7,8$ tetramethyl-1,10-phenanthroline ( $2 \mathrm{mg}, 0.008 \mathrm{mmol}), \mathrm{Zn}$ dust $(156 \mathrm{mg}, 2.4 \mathrm{mmol})$ and $\mathrm{NaOAc}(16.4 \mathrm{mg}$, $0.2 \mathrm{mmol})$ were added to $0.8 \mathrm{~mL} 2 \mathrm{wt} \%$ TPGS- $750-\mathrm{M} / \mathrm{H}_{2} \mathrm{O}$ with $\mathrm{MeCN}(0.08 \mathrm{~mL})$ as co-solvent, and the mixture was vigorously stirred at $\mathrm{rt}$. After $16 \mathrm{~h}$, EtOAc was added and the mixture stirred for $2 \mathrm{~min}$ at $\mathrm{rt}$. Stirring was then stopped and the organic layer was decanted via pipette. The organic layer was passed through a very small silica plug. Yields were determined by GC using naphthalene as internal standard. 
No iron salts. To a flame dried $4 \mathrm{~mL}$ microwave reaction vial, 6-bromohex-1-ene (195.7 mg, 1.2 mmol), tert-butyl acrylate (307.7 mg, $2.4 \mathrm{mmol}$ ), Zn dust (156 mg, $2.4 \mathrm{mmol})$ and NaOAc (16.4 mg, 0.2 mmol) were added to $0.8 \mathrm{~mL} 2$ wt $\%$ TPGS-750-M/ $\mathrm{H}_{2} \mathrm{O}$ with $\mathrm{MeCN}(0.08 \mathrm{~mL})$ as co-solvent, and the mixture was vigorously stirred at $\mathrm{rt}$. After $16 \mathrm{~h}$, EtOAc was added and the mixture stirred for $2 \mathrm{~min}$ at $\mathrm{rt}$. Stirring was then stopped and the organic layer was decanted via pipette. The organic layer was passed through a very small silica plug. Yields were determined by GC using naphthalene as internal standard.

\section{e) Radical scavenger test}

2-Vinylpyridine (21 mg, $0.2 \mathrm{mmol}$ ), ethyl 3-bromopropanoate (108.6 mg, $0.6 \mathrm{mmol}$ ), $\mathrm{Fe}\left(\mathrm{NH}_{4}\right)_{2}\left(\mathrm{SO}_{4}\right)_{2} \cdot 6 \mathrm{H}_{2} \mathrm{O}$ (1.6 mg, $\left.0.004 \mathrm{mmol}\right), 3,4,7,8$-tetramethyl-1,10-phenanthroline $(1 \mathrm{mg}, 0.004$ $\mathrm{mmol}$ ), Zn dust (78 mg, $1.2 \mathrm{mmol}$ ), radical scavenger (0.01 mmol or $0.2 \mathrm{mmol}$ ) and $\mathrm{NaOAc}(8.2 \mathrm{mg}, 0.1$ $\mathrm{mmol})$, were added to $0.4 \mathrm{~mL} 2 \mathrm{wt} \% \mathrm{TPGS}-750-\mathrm{M} / \mathrm{H}_{2} \mathrm{O}$ with $\mathrm{MeCN}(0.04 \mathrm{~mL})$ as co-solvent were reacted at rt for $16 \mathrm{~h}$.

2-Vinylpyridine (21 $\mathrm{mg}, \quad 0.2 \mathrm{mmol}), \quad$ 1-bromoadamantane $\left(\begin{array}{lllll}129.1 & \mathrm{mg}, & 0.6 \mathrm{mmol}\end{array}\right)$, $\mathrm{Fe}\left(\mathrm{NH}_{4}\right)_{2}\left(\mathrm{SO}_{4}\right)_{2} \cdot 6 \mathrm{H}_{2} \mathrm{O}$ (1.6 mg, $\left.0.004 \mathrm{mmol}\right)$, 3,4,7,8-tetramethyl-1,10-phenanthroline (1 mg, 0.004 $\mathrm{mmol})$, Zn dust (78 mg, $1.2 \mathrm{mmol})$, radical scavenger ( $0.01 \mathrm{mmol}$ or $0.2 \mathrm{mmol})$ and $\mathrm{NaOAc}(8.2 \mathrm{mg}, 0.1$ mmol) were added to $0.4 \mathrm{~mL} 2 \mathrm{wt} \% \mathrm{TPGS}-750-\mathrm{M} / \mathrm{H}_{2} \mathrm{O}$ with $\mathrm{MeCN}(0.04 \mathrm{~mL})$ as co-solvent were reacted at $45^{\circ} \mathrm{C}$ for $16 \mathrm{~h}$. 


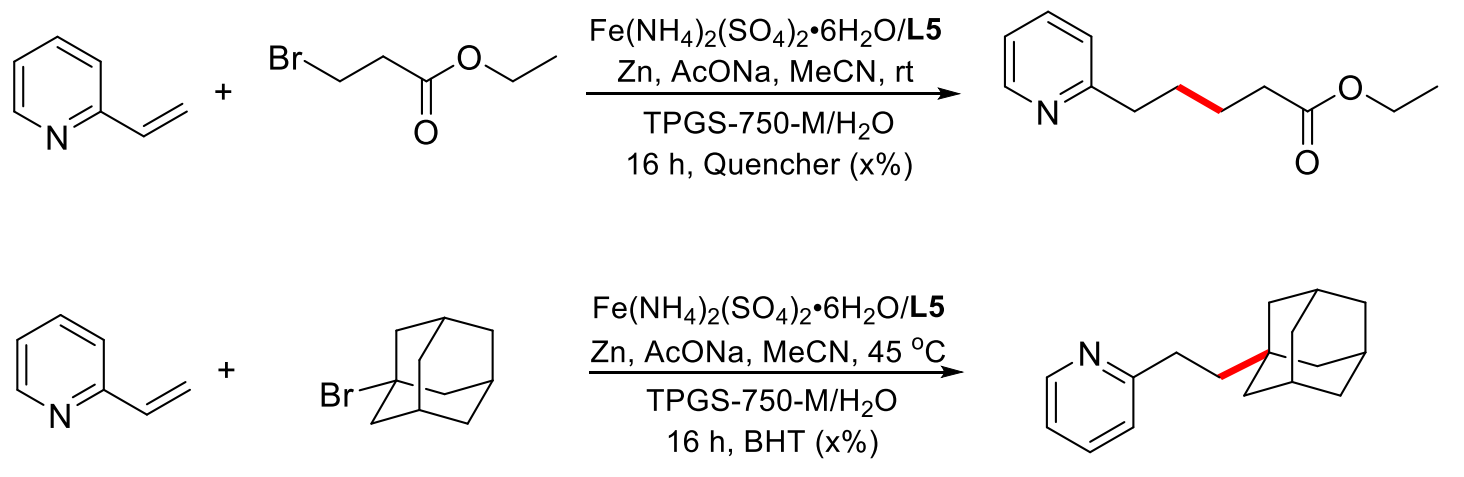

\begin{tabular}{|c|c|c|c|c|}
\hline Entry $^{a}$ & Reaction & Quencher & $x$ & Isolated yield (\%) \\
\hline 1 & 1 & none & 0 & 70 \\
\hline 2 & 1 & TEMPO & 5 & 71 \\
\hline 3 & 1 & galvonoxyl & 5 & 63 \\
\hline 4 & 1 & BHT & 5 & 72 \\
\hline 5 & 1 & TEMPO & 100 & 57 \\
\hline 6 & 1 & galvonoxyl & 100 & 58 \\
\hline 7 & 1 & BHT & 100 & 70 \\
\hline 8 & 2 & $\mathrm{BHT}$ & 0 & 63 \\
\hline 9 & 2 & $\mathrm{BHT}$ & 100 & 53 \\
\hline $\begin{array}{l}{ }^{\mathrm{a}} \text { Reaction } \\
\mathrm{Fe}\left(\mathrm{NH}_{4}\right)_{2}(\mathrm{~S} \\
\mathrm{mmol}), \mathrm{MeC} \\
(0.4 \mathrm{~mL})\end{array}$ & $\begin{array}{l}\text { itions: } \\
\mathrm{H}_{2} \mathrm{O}(0.00 \\
4 \mathrm{~mL}) \text {, qu }\end{array}$ & $\begin{array}{l}\text { n } \quad(0.2 \quad \mathrm{mmc} \\
\mathrm{hol}), \mathbf{L} 5(0.004 \\
\text { r }(0.01 \mathrm{mmol} \text { or }\end{array}$ & $\begin{array}{l}\text { hatic } \\
\text { In dus } \\
\text { l) and }\end{array}$ & $\begin{array}{l}\text { mide }(0.6 \mathrm{mmol}) \text {, } \\
\text { ? } \mathrm{mmol}), \mathrm{AcONa}(0.1 \\
\% \text { TPGS-750-M/ } \mathrm{H}_{2} \mathrm{O}\end{array}$ \\
\hline
\end{tabular}

However, it was determined that neither TEMPO nor galvonoxyl free radical was stable under such reducing conditions given the large excess of $\mathrm{Zn}$ dust present. Both are reduced even with only one equivalent Zn dust in the reaction. In addition, BHT did not dissolve well in the aqueous phase which may influence its reactivity as a scavenger. Hence, based on these observations it is difficult to make a definitive conclusion as to the involvement of a radical intermediate in these reactions.

\section{Analytical data for products}




\section{1-(t-Butyl)-4-(5-phenoxypentyl)benzene (1)}

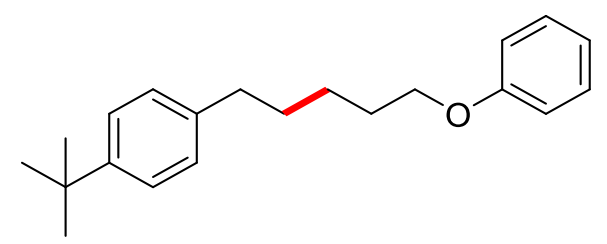

1-(t-Butyl)-4-vinylbenzene (32 mg, $0.2 \mathrm{mmol})$, (3-bromopropoxy)benzene (129.1 mg, $0.6 \mathrm{mmol})$, $\mathrm{Fe}(\mathrm{acac})_{3}$ (1.4 mg, $\left.0.004 \mathrm{mmol}\right)$, 3,4,7,8-tetramethyl-1,10-phenanthroline (1 mg, $\left.0.004 \mathrm{mmol}\right)$, Zn dust (78 mg, $1.2 \mathrm{mmol})$ and $\mathrm{NaCl}(70.2 \mathrm{mg}, 1.2 \mathrm{mmol})$, in $0.4 \mathrm{~mL} 2$ wt \% TPGS-750-M/ $\mathrm{H}_{2} \mathrm{O}$ were reacted at rt for $16 \mathrm{~h}$ yielding $44.2 \mathrm{mg}(75 \%)$ of 1-(t-butyl)-4-(5-phenoxypentyl)benzene as a colorless oil (hexane/EtOAc: 95/5).

${ }^{1} \mathrm{H}$ NMR $\left(500 \mathrm{MHz}, \mathrm{CDCl}_{3}\right) \delta$ 7.34-7.26 (m, 4H), $7.13(\mathrm{~d}, J=8.1 \mathrm{~Hz}, 2 \mathrm{H}), 6.94(\mathrm{t}, J=7.3 \mathrm{~Hz}$, $1 \mathrm{H}), 6.90(\mathrm{~d}, J=8.3 \mathrm{~Hz}, 2 \mathrm{H}), 3.97(\mathrm{t}, J=6.5 \mathrm{~Hz}, 2 \mathrm{H}), 2.63(\mathrm{t}, 2 \mathrm{H}), 1.90-1.78(\mathrm{~m}, 2 \mathrm{H}), 1.76-1.66(\mathrm{~m}, 2 \mathrm{H})$, $1.58-1.49(\mathrm{~m}, 3 \mathrm{H}), 1.32(\mathrm{~s}, 9 \mathrm{H})$.

${ }^{13} \mathrm{C} \mathrm{NMR}\left(126 \mathrm{MHz}, \mathrm{CDCl}_{3}\right) \delta 159.2,148.6,139.6,129.5,128.2,125.3,120.6,114.6,77.4,77.2$, $76.9,67.9,35.5,34.5,31.6,31.4,29.3,26.0$.

HRMS(EI): Calcd. for $\mathrm{C}_{21} \mathrm{H}_{28} \mathrm{O}[\mathrm{M}]^{+}$296.2140. Found: 296.2141 .

\section{1-Bromo-4-(5-phenoxypentyl)benzene (2)}

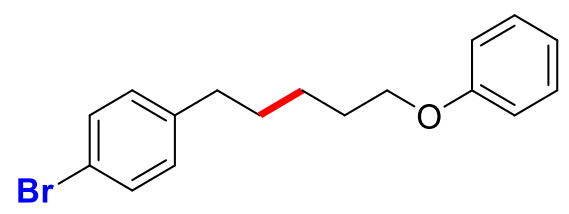

1-Bromo-4-vinylbenzene (36.6 mg, $0.2 \mathrm{mmol}$ ), (3-bromopropoxy)benzene (129.1 mg, $0.6 \mathrm{mmol}$ ), $\mathrm{Fe}\left(\mathrm{NH}_{4}\right)_{2}\left(\mathrm{SO}_{4}\right)_{2} \cdot 6 \mathrm{H}_{2} \mathrm{O}(8 \mathrm{mg}, 0.02 \mathrm{mmol})$, 3,4,7,8-tetramethyl-1,10-phenanthroline (5 mg, $\left.0.02 \mathrm{mmol}\right)$, Zn dust $(78 \mathrm{mg}, 1.2 \mathrm{mmol})$ and $\mathrm{NaOAc}(8.2 \mathrm{mg}, 0.1 \mathrm{mmol})$, in $0.4 \mathrm{~mL} 2$ wt \% TPGS-750-M/ $\mathrm{H}_{2} \mathrm{O}$ with $\mathrm{MeCN}(0.04 \mathrm{~mL})$ as co-solvent were reacted at $\mathrm{rt}$ for $16 \mathrm{~h}$ yielding $45.5 \mathrm{mg}$ (72\%) of 1-bromo-4-(5phenoxypentyl)benzene as a colorless oil (hexane/EtOAc: 95/5). 
${ }^{1} \mathrm{H}$ NMR $\left(500 \mathrm{MHz}, \mathrm{CDCl}_{3}\right) \delta 7.39(\mathrm{~d}, J=6.9 \mathrm{~Hz}, 2 \mathrm{H}), 7.31-7.24(\mathrm{~m}, 2 \mathrm{H}), 7.06(\mathrm{~d}, J=7.1 \mathrm{~Hz}$, 2H), $6.93(\mathrm{t}, J=7.3 \mathrm{~Hz}, 1 \mathrm{H}), 6.89(\mathrm{~d}, J=7.7 \mathrm{~Hz}, 2 \mathrm{H}), 3.95(\mathrm{t}, J=6.4 \mathrm{~Hz}, 2 \mathrm{H}), 2.60(\mathrm{t}, J=7.6 \mathrm{~Hz}, 2 \mathrm{H})$, 1.91-1.73 (m, 2H), 1.71-1.63 (m, 2H), 1.54-1.45 (m, 2H).

${ }^{13} \mathrm{C} \mathrm{NMR}\left(126 \mathrm{MHz}, \mathrm{CDCl}_{3}\right) \delta 159.2,141.6,131.5,130.3,129.6,120.7,119.5,114.6,67.8,35.4$, $31.2,29.3,25.8$

HRMS(EI): Calcd. for $\mathrm{C}_{17} \mathrm{H}_{19} \mathrm{BrO}[\mathrm{M}]^{+}$318.0619. Found: 318.0611 .

\section{1-(5-Phenoxypentyl)-4-(trifluoromethyl)benzene (3)}

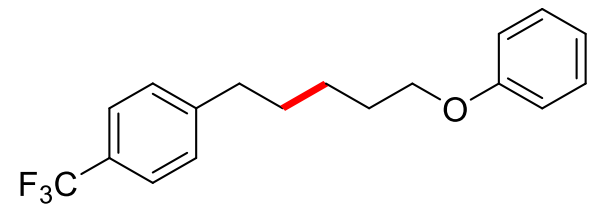

1-(Trifluoromethyl)-4-vinylbenzene (34.4 mg, $0.2 \mathrm{mmol}$ ), (3-bromopropoxy)benzene (129.1 mg, $0.6 \mathrm{mmol}), \mathrm{Fe}\left(\mathrm{NH}_{4}\right)_{2}\left(\mathrm{SO}_{4}\right)_{2} \cdot 6 \mathrm{H}_{2} \mathrm{O}$ (1.6 mg, $\left.0.004 \mathrm{mmol}\right)$, 3,4,7,8-tetramethyl-1,10-phenanthroline (1 mg, $0.004 \mathrm{mmol}$ ), $\mathrm{Zn}$ dust (78 mg, $1.2 \mathrm{mmol})$ and NaOAc (8.2 mg, $0.1 \mathrm{mmol})$, in $0.4 \mathrm{~mL} 2 \mathrm{wt} \% \mathrm{TPGS}-750-$ $\mathrm{M} / \mathrm{H}_{2} \mathrm{O}$ with $\mathrm{MeCN}(0.04 \mathrm{~mL})$ as co-solvent were reacted at $\mathrm{rt}$ for $16 \mathrm{~h}$ yielding $43.5 \mathrm{mg}(71 \%)$ of 1 -(5phenoxypentyl)-4-(trifluoromethyl)benzene as a slight yellow oil (hexane/EtOAc: 90/10).

${ }^{1} \mathrm{H}$ NMR (500 MHz, $\left.\mathrm{CDCl}_{3}\right) \delta 7.53(\mathrm{~d}, J=8.0 \mathrm{~Hz}, 2 \mathrm{H}), 7.34-7.25(\mathrm{~m}, 3 \mathrm{H}), 6.98-6.86(\mathrm{~m}, 4 \mathrm{H})$, $3.96(\mathrm{t}, J=6.4 \mathrm{~Hz}, 2 \mathrm{H}), 2.70(\mathrm{t}, J=7.7 \mathrm{~Hz}, 2 \mathrm{H}), 1.87-1.77(\mathrm{~m}, 2 \mathrm{H}), 1.76-1.67(\mathrm{~m}, 2 \mathrm{H}), 1.55-1.49(\mathrm{~m}, 2 \mathrm{H})$.

${ }^{13} \mathrm{C} \mathrm{NMR}\left(126 \mathrm{MHz}, \mathrm{CDCl}_{3}\right) \delta 159.1,146.7,129.6,128.8,128.6\left(\mathrm{q},{ }^{1} J_{(\mathrm{C}-\mathrm{F})}=67 \mathrm{~Hz}\right), 125.4(\mathrm{q}$, $\left.{ }^{1} J_{(\mathrm{C}-\mathrm{F})}=3.8 \mathrm{~Hz}\right), 124.5\left(\mathrm{q},{ }^{1} J_{(\mathrm{C}-\mathrm{F})}=272 \mathrm{~Hz}\right), 120.7,114.6,67.7,35.8,31.1,29.3,25.9$.

${ }^{19} \mathrm{~F}$ NMR $\left(376 \mathrm{MHz}, \mathrm{CDCl}_{3}\right) \delta-62.28$.

HRMS(EI): Calcd. for $\mathrm{C}_{18} \mathrm{H}_{19} \mathrm{~F}_{3} \mathrm{O}[\mathrm{M}]^{+}$308.1388. Found: 308.1385 .

Ethyl 5-(4-cyanophenyl)pentanoate (4)<smiles>CCOC(=O)CCCCc1ccc(C#N)cc1</smiles> 
4-Vinylbenzonitrile (25.8 mg, $0.2 \mathrm{mmol}$ ), ethyl 3-bromopropanoate (108.6 mg, $0.6 \mathrm{mmol})$, $\mathrm{Fe}\left(\mathrm{NH}_{4}\right)_{2}\left(\mathrm{SO}_{4}\right)_{2} \cdot 6 \mathrm{H}_{2} \mathrm{O}(1.6 \mathrm{mg}, 0.004 \mathrm{mmol}), 3,4,7,8$-tetramethyl-1,10-phenanthroline (1 mg, 0.004 mmol), Zn dust (78 mg, $1.2 \mathrm{mmol}$ ) and NaOAc ( $8.2 \mathrm{mg}, 0.1 \mathrm{mmol})$, in $0.4 \mathrm{~mL} 2 \mathrm{wt} \%$ TPGS-750-M/ $\mathrm{H}_{2} \mathrm{O}$ with $\mathrm{MeCN}(0.04 \mathrm{~mL})$ as co-solvent were reacted at $\mathrm{rt}$ for $16 \mathrm{~h}$ yielding $36.9 \mathrm{mg}(80 \%)$ of ethyl 5-(4cyanophenyl)pentanoate as a slight yellow oil (hexane/EtOAc: 90/10).

${ }^{1} \mathrm{H} \mathrm{NMR}\left(500 \mathrm{MHz}, \mathrm{CDCl}_{3}\right) \delta 7.56(\mathrm{~d}, J=8.2 \mathrm{~Hz}, 2 \mathrm{H}), 7.27(\mathrm{~d}, J=7.9 \mathrm{~Hz}, 2 \mathrm{H}), 4.11(\mathrm{q}, J=7.1$ $\mathrm{Hz}, 2 \mathrm{H}), 2.74-2.62(\mathrm{~m}, 2 \mathrm{H}), 2.38-2.26(\mathrm{~m}, 2 \mathrm{H}), 1.72-1.58(\mathrm{~m}, 4 \mathrm{H}), 1.24(\mathrm{t}, J=7.1 \mathrm{~Hz}, 3 \mathrm{H})$.

${ }^{13} \mathrm{C}$ NMR $\left(126 \mathrm{MHz}, \mathrm{CDCl}_{3}\right) \delta 173.5,147.9,132.3,129.3,119.2,109.9,60.5,35.9,34.1,30.4$, $24.6,14.4$.

HRMS(EI): Calcd. for $\mathrm{C}_{14} \mathrm{H}_{17} \mathrm{NO}_{2}[\mathrm{M}]^{+}$231.1259. Found: 231.1260 .

\section{Ethyl 5-(4-(t-butyl)phenyl)pentanoate (5)}

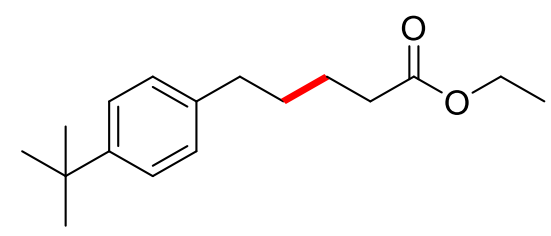

1-(t-Butyl)-4-vinylbenzene (32 mg, $0.2 \mathrm{mmol})$, ethyl 3-bromopropanoate (108.6 mg, $0.6 \mathrm{mmol})$, $\mathrm{Fe}\left(\mathrm{NH}_{4}\right)_{2}\left(\mathrm{SO}_{4}\right)_{2} \cdot 6 \mathrm{H}_{2} \mathrm{O}(1.6 \mathrm{mg}, 0.004 \mathrm{mmol}), 3,4,7,8$-tetramethyl-1,10-phenanthroline (1 mg, 0.004 mmol), Zn dust (78 mg, $1.2 \mathrm{mmol}$ ) and NaOAc (8.2 mg, $0.1 \mathrm{mmol})$, in $0.4 \mathrm{~mL} 2 \mathrm{wt} \%$ TPGS-750-M/ $\mathrm{H}_{2} \mathrm{O}$ with $\mathrm{MeCN}(0.04 \mathrm{~mL})$ as co-solvent were reacted at $\mathrm{rt}$ for $16 \mathrm{~h}$ yielding $35.1 \mathrm{mg}(67 \%)$ of ethyl 5-(4-(tertbutyl)phenyl)pentanoate as a slight yellow oil (hexane/EtOAc: 90/10).

${ }^{1} \mathrm{H}$ NMR $\left(500 \mathrm{MHz}, \mathrm{CDCl}_{3}\right) \delta 7.30(\mathrm{~d}, J=8.2 \mathrm{~Hz}, 2 \mathrm{H}), 7.11(\mathrm{~d}, J=8.1 \mathrm{~Hz}, 2 \mathrm{H}), 4.12(\mathrm{q}, J=7.1$ $\mathrm{Hz}, 2 \mathrm{H}), 2.60$ (t, $J=7.2 \mathrm{~Hz}, 2 \mathrm{H}), 2.33(\mathrm{t}, J=7.0 \mathrm{~Hz}, 2 \mathrm{H}), 1.74-1.62(\mathrm{~m}, 4 \mathrm{H}), 1.31(\mathrm{~s}, 9 \mathrm{H}), 1.25$ (t, $J=7.1$ $\mathrm{Hz}, 3 \mathrm{H})$.

${ }^{13} \mathrm{C}$ NMR $\left(126 \mathrm{MHz}, \mathrm{CDCl}_{3}\right) \delta 173.8,148.7,139.2,128.2,125.3,77.4,77.2,76.9,60.4,35.2$, $34.5,34.4,31.6,31.0,24.8,14.4$.

HRMS(ESI): Calcd. for $\mathrm{C}_{17} \mathrm{H}_{26} \mathrm{O}_{2} \mathrm{Na}[\mathrm{M}+\mathrm{Na}]^{+}$285.1830. Found: 285.1838.

\section{Ethyl 5-(naphthalen-1-yl)pentanoate (6)}




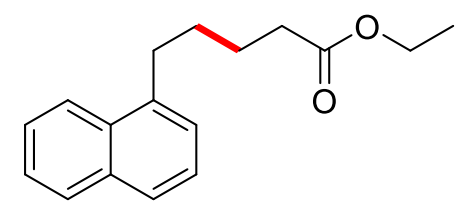

1-Vinylnaphthalene (30.8 mg, $0.2 \mathrm{mmol})$, ethyl 3-bromopropanoate (108.6 mg, $0.6 \mathrm{mmol})$, $\mathrm{Fe}\left(\mathrm{NH}_{4}\right)_{2}\left(\mathrm{SO}_{4}\right)_{2} \cdot 6 \mathrm{H}_{2} \mathrm{O}$ (1.6 mg, $\left.0.004 \mathrm{mmol}\right)$, 3,4,7,8-tetramethyl-1,10-phenanthroline (1 mg, 0.004 $\mathrm{mmol}$ ), $\mathrm{Zn}$ dust (78 mg, $1.2 \mathrm{mmol}$ ) and $\mathrm{NaOAc}(8.2 \mathrm{mg}, 0.1 \mathrm{mmol})$, in $0.4 \mathrm{~mL} 2 \mathrm{wt} \% \mathrm{TPGS}-750-\mathrm{M} / \mathrm{H}_{2} \mathrm{O}$ with $\mathrm{MeCN}(0.04 \mathrm{~mL})$ as co-solvent were reacted at $\mathrm{rt}$ for $16 \mathrm{~h}$ yielding $22.9 \mathrm{mg}(45 \%)$ of ethyl 5(naphthalen-1-yl)pentanoate as a colorless oil (hexane/EtOAc: 90/10).

${ }^{1} \mathrm{H}$ NMR $\left(500 \mathrm{MHz}, \mathrm{CDCl}_{3}\right) \delta$ 7.82-7.74 (m, 3H), 7.63-7.59 (m, 1H), 7.48-7.39 (m, 2H), 7.34$7.30(\mathrm{~m}, 1 \mathrm{H}), 4.13(\mathrm{q}, J=7.1 \mathrm{~Hz}, 2 \mathrm{H}), 2.80(\mathrm{t}, J=7.2 \mathrm{~Hz}, 2 \mathrm{H}), 2.35$ (t, $J=7.1 \mathrm{~Hz}, 2 \mathrm{H}), 1.80-1.67$ (m, $4 \mathrm{H}), 1.25(\mathrm{t}, J=7.1 \mathrm{~Hz}, 3 \mathrm{H})$.

${ }^{13} \mathrm{C}$ NMR $\left(126 \mathrm{MHz}, \mathrm{CDCl}_{3}\right) \delta 173.8,139.8,133.8,132.1,128.0,127.7,127.5,127.4,126.5$, 126.0, 125.2, 60.4, 35.9, 34.4, 30.9, 24.8, 14.4 .

HRMS(ESI): Calcd. for $\mathrm{C}_{17} \mathrm{H}_{20} \mathrm{O}_{2} \mathrm{Na}[\mathrm{M}+\mathrm{Na}]^{+}$279.1361. Found: 279.1364 .

\section{1-Chloro-2-(5-phenoxypentyl)benzene (7)}

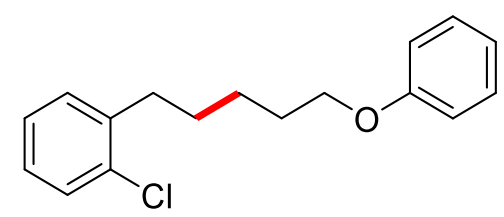

2-Chlorostyrene (27.6 mg, $0.2 \mathrm{mmol}$ ), (3-bromopropoxy)benzene (129.1 mg, $0.6 \mathrm{mmol}$ ), $\mathrm{Fe}\left(\mathrm{NH}_{4}\right)_{2}\left(\mathrm{SO}_{4}\right)_{2} \cdot 6 \mathrm{H}_{2} \mathrm{O}$ (1.6 mg, $\left.0.004 \mathrm{mmol}\right), 3,4,7,8$-tetramethyl-1,10-phenanthroline (1 mg, 0.004 $\mathrm{mmol}$ ), $\mathrm{Zn}$ dust (78 mg, $1.2 \mathrm{mmol}$ ) and $\mathrm{NaOAc}(8.2 \mathrm{mg}, 0.1 \mathrm{mmol})$, in $0.4 \mathrm{~mL} 2 \mathrm{wt} \%$ TPGS-750-M/ $\mathrm{H}_{2} \mathrm{O}$ with $\mathrm{MeCN}(0.04 \mathrm{~mL})$ as co-solvent were reacted at $\mathrm{rt}$ for $16 \mathrm{~h}$ yielding $40.1 \mathrm{mg}$ (73\%) of 1-chloro-2-(5phenoxypentyl)benzene as a colorless oil (hexane/EtOAc: 90/10).

${ }^{1} \mathrm{H}$ NMR $\left(500 \mathrm{MHz}, \mathrm{CDCl}_{3}\right) \delta$ 7.45-7.09 (m, 6H), 7.02-6.87 (m, 3H), $3.98(\mathrm{t}, J=6.5 \mathrm{~Hz}, 2 \mathrm{H})$, 2.83-2.73 (m, 2H), 1.91-1.81 (m, 2H), 1.76-1.66 (m, 2H), 1.62-1.51 (m, 2H).

${ }^{13} \mathrm{C}$ NMR $\left(126 \mathrm{MHz}, \mathrm{CDCl}_{3}\right) \delta 159.2,140.2,134.0,130.5,129.6,129.6,127.3,126.8,120.6$, $114.6,67.9,33.7,29.7,29.3,26.0$. 
HRMS(EI): Calcd. for $\mathrm{C}_{17} \mathrm{H}_{19} \mathrm{ClO}[\mathrm{M}]^{+} 274.1125$. Found: 274.1123 .

\section{Ethyl 5-(3-(benzofuran-2-yl)phenyl)pentanoate (8)}

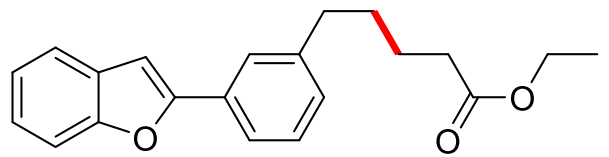

2-(3-Vinylphenyl)benzofuran $(44.0 \mathrm{mg}, 0.2 \mathrm{mmol})$, ethyl 3-bromopropanoate (108.6 mg, 0.6 mmol), $\mathrm{Fe}\left(\mathrm{NH}_{4}\right)_{2}\left(\mathrm{SO}_{4}\right)_{2} \cdot 6 \mathrm{H}_{2} \mathrm{O}$ (1.6 mg, $\left.0.004 \mathrm{mmol}\right), 3,4,7,8$-tetramethyl-1,10-phenanthroline (1 mg, $0.004 \mathrm{mmol}), \mathrm{Zn}$ dust (78 mg, $1.2 \mathrm{mmol})$ and $\mathrm{NaOAc}(8.2 \mathrm{mg}, 0.1 \mathrm{mmol})$, in $0.4 \mathrm{~mL} 2 \mathrm{wt} \%$ TPGS-750$\mathrm{M} / \mathrm{H}_{2} \mathrm{O}$ with $\mathrm{MeCN}(0.04 \mathrm{~mL})$ as co-solvent were reacted at $\mathrm{rt}$ for $16 \mathrm{~h}$ yielding $30.1 \mathrm{mg}(47 \%)$ of ethyl 5-(3-(benzofuran-2-yl)phenyl)pentanoate as a colorless oil (hexane/EtOAc: 90/10).

${ }^{1} \mathrm{H}$ NMR $\left(500 \mathrm{MHz}, \mathrm{CDCl}_{3}\right) \delta$ 7.73-7.66 (m, 2H), $7.58(\mathrm{~d}, J=7.6 \mathrm{~Hz}, 1 \mathrm{H}), 7.53(\mathrm{~d}, J=8.0 \mathrm{~Hz}$, 1H), $7.36(\mathrm{t}, J=7.9 \mathrm{~Hz}, 1 \mathrm{H}), 7.30-7.26(\mathrm{~m}, 1 \mathrm{H}), 7.23(\mathrm{t}, J=7.4 \mathrm{~Hz}, 1 \mathrm{H}), 7.17(\mathrm{~d}, J=7.4 \mathrm{~Hz}, 1 \mathrm{H}), 7.02$ (s, 1H), 4.13 (q, $J=7.1 \mathrm{~Hz}, 2 \mathrm{H}), 2.75-2.65(\mathrm{~m}, 2 \mathrm{H}), 2.40-2.31(\mathrm{~m}, 2 \mathrm{H}), 1.80-1.62(\mathrm{~m}, 4 \mathrm{H}), 1.25(\mathrm{t}, J=7.1$ $\mathrm{Hz}, 3 \mathrm{H})$.

${ }^{13} \mathrm{C}$ NMR $\left(126 \mathrm{MHz}, \mathrm{CDCl}_{3}\right) \delta 173.8,156.2,155.0,142.9,130.6,129.4,128.9,128.9,125.0$, 124.3, 123.0, 122.7, 121.0, 111.3, 101.4, 60.4, 35.8, 34.4, 31.0, 24.8, 14.4 .

HRMS(ESI): Calcd. for $\mathrm{C}_{21} \mathrm{H}_{22} \mathrm{O}_{3} \mathrm{Na}[\mathrm{M}+\mathrm{Na}]^{+}$345.1467. Found: 345.1471 .

\section{Ethyl 5-(2,4-dimethylphenyl)pentanoate (9)}

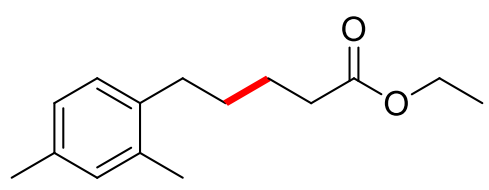

2,4-dimethyl-1-vinylbenzene (26.4 mg, $0.2 \mathrm{mmol}$ ), ethyl 3-bromopropanoate (108.6 mg, 0.6 mmol), $\mathrm{Fe}\left(\mathrm{NH}_{4}\right)_{2}\left(\mathrm{SO}_{4}\right)_{2} \cdot 6 \mathrm{H}_{2} \mathrm{O}$ (1.6 mg, $\left.0.004 \mathrm{mmol}\right)$, 3,4,7,8-tetramethyl-1,10-phenanthroline (1 mg, $0.004 \mathrm{mmol}), \mathrm{Zn}$ dust $(78 \mathrm{mg}, 1.2 \mathrm{mmol})$ and NaOAc $(8.2 \mathrm{mg}, 0.1 \mathrm{mmol})$, in $0.4 \mathrm{~mL} 2 \mathrm{wt} \%$ TPGS-750$\mathrm{M} / \mathrm{H}_{2} \mathrm{O}$ with $\mathrm{MeCN}(0.04 \mathrm{~mL})$ as co-solvent were reacted at $\mathrm{rt}$ for $16 \mathrm{~h}$ yielding $28.5 \mathrm{mg}(61 \%)$ of ethyl 5-(2,4-dimethylphenyl)pentanoate as a yellow oil (hexane/EtOAc: 90/10). 
${ }^{1} \mathrm{H}$ NMR $\left(500 \mathrm{MHz}, \mathrm{CDCl}_{3}\right) \delta 7.04(\mathrm{dd}, J=7.6,1.8 \mathrm{~Hz}, 1 \mathrm{H}), 7.00-6.93(\mathrm{~m}, 2 \mathrm{H}), 4.15(\mathrm{qd}, J=$ 7.1, 2.1 Hz, 2H), 2.60 (t, $J=8.7 \mathrm{~Hz}, 2 \mathrm{H}), 2.36$ (td, $J=7.5,2.0 \mathrm{~Hz}, 2 \mathrm{H}), 2.31$ (s, 3H), 2.29 (s, 3H), 1.74 $(\mathrm{p}, J=7.4 \mathrm{~Hz}, 2 \mathrm{H}), 1.61(\mathrm{p}, J=7.0,6.5 \mathrm{~Hz}, 2 \mathrm{H}), 1.28(\mathrm{td}, J=7.1,2.1 \mathrm{~Hz}, 3 \mathrm{H})$.

${ }^{13} \mathrm{C}$ NMR $\left(126 \mathrm{MHz}, \mathrm{CDCl}_{3}\right) \delta 173.73,137.38,135.69,135.38,131.07,128.84,126.63,60.31$, $34.37,32.64,30.01,25.08,20.98,19.29,14.36$.

HRMS(ESI): Calcd. for $\mathrm{C}_{15} \mathrm{H}_{22} \mathrm{O}_{2} \mathrm{Na}[\mathrm{M}+\mathrm{Na}]^{+}$257.1518. Found: 257.1509.

\section{Ethyl 5-(3-acetylphenyl)pentanoate (10)}<smiles>CCOC(=O)CCCCc1cccc(C(C)=O)c1</smiles>

1-(3-Vinylphenyl)ethanone (29.3 mg, $0.2 \mathrm{mmol})$, ethyl 3-bromopropanoate (108.6 mg, $0.6 \mathrm{mmol})$, $\mathrm{Fe}\left(\mathrm{NH}_{4}\right)_{2}\left(\mathrm{SO}_{4}\right)_{2} \cdot 6 \mathrm{H}_{2} \mathrm{O}(1.6 \mathrm{mg}, 0.004 \mathrm{mmol})$, 3,4,7,8-tetramethyl-1,10-phenanthroline (1 mg, 0.004 mmol), Zn dust (78 mg, $1.2 \mathrm{mmol})$ and $\mathrm{NaOAc}(8.2 \mathrm{mg}, 0.1 \mathrm{mmol})$, in $0.4 \mathrm{~mL} 2 \mathrm{wt} \% \mathrm{TPGS}-750-\mathrm{M} / \mathrm{H}_{2} \mathrm{O}$ with $\mathrm{MeCN}(0.04 \mathrm{~mL})$ as co-solvent were reacted at $\mathrm{rt}$ for $16 \mathrm{~h}$ yielding $27.8 \mathrm{mg}(56 \%)$ of ethyl 5-(3acetylphenyl)pentanoate as a colorless oil (hexane/EtOAc: 80/20).

${ }^{1} \mathrm{H}$ NMR $\left(500 \mathrm{MHz}, \mathrm{CDCl}_{3}\right) \delta 7.80-7.73(\mathrm{~m}, 2 \mathrm{H}), 7.41-7.33(\mathrm{~m}, 2 \mathrm{H}), 4.11(\mathrm{q}, J=7.1 \mathrm{~Hz}, 2 \mathrm{H})$, $2.68(\mathrm{t}, J=6.9 \mathrm{~Hz}, 2 \mathrm{H}), 2.59(\mathrm{~s}, 3 \mathrm{H}), 2.32(\mathrm{t}, J=6.8 \mathrm{~Hz}, 2 \mathrm{H}), 1.67(\mathrm{p}, J=3.7 \mathrm{~Hz}, 4 \mathrm{H}), 1.24(\mathrm{t}, J=7.1 \mathrm{~Hz}$ $3 \mathrm{H})$.

${ }^{13} \mathrm{C}$ NMR $\left(126 \mathrm{MHz}, \mathrm{CDCl}_{3}\right) \delta 198.43,173.60,142.79,137.38,133.33,128.65,128.17,126.17$, $60.37,35.54,34.22,30.87,26.79,24.63,14.35$.

HRMS(ESI): Calcd. for $\mathrm{C}_{15} \mathrm{H}_{20} \mathrm{O}_{3} \mathrm{Na}[\mathrm{M}+\mathrm{Na}]^{+}$271.1310. Found: 271.1322.

Ethyl 5-(2-allylphenyl)pentanoate (11)<smiles>C=CCc1ccccc1CCCCC(=O)OCC</smiles> 
1-Allyl-2-vinylbenzene $(28.8 \mathrm{mg}, 0.2 \mathrm{mmol})$, ethyl 3-bromopropanoate (108.6 mg, $0.6 \mathrm{mmol})$, $\mathrm{Fe}\left(\mathrm{NH}_{4}\right)_{2}\left(\mathrm{SO}_{4}\right)_{2} \cdot 6 \mathrm{H}_{2} \mathrm{O}(1.6 \mathrm{mg}, 0.004 \mathrm{mmol}), 3,4,7,8$-tetramethyl-1,10-phenanthroline (1 mg, 0.004 mmol), Zn dust (78 mg, $1.2 \mathrm{mmol})$ and NaOAc (8.2 mg, $0.1 \mathrm{mmol})$ in $0.4 \mathrm{~mL} 2$ wt \% TPGS-750-M/ $\mathrm{H}_{2} \mathrm{O}$ with $\mathrm{MeCN}(0.04 \mathrm{~mL})$ as co-solvent were reacted at $\mathrm{rt}$ for $16 \mathrm{~h}$ yielding $30.5 \mathrm{mg}(62 \%)$ of ethyl 5-(2allylphenyl)pentanoate as a faint yellow oil (hexane/EtOAc: 90/10).

${ }^{1} \mathrm{H}$ NMR $\left(500 \mathrm{MHz}, \mathrm{CDCl}_{3}\right) \delta 7.16(\mathrm{~s}, 4 \mathrm{H}), 5.97(\mathrm{ddt}, J=16.5,10.1,6.3 \mathrm{~Hz}, 1 \mathrm{H}), 5.07(\mathrm{dq}, J=$ $10.1,1.5 \mathrm{~Hz}, 1 \mathrm{H}), 5.01(\mathrm{dq}, J=17.1,1.7 \mathrm{~Hz}, 1 \mathrm{H}), 4.14(\mathrm{q}, J=7.1 \mathrm{~Hz}, 2 \mathrm{H}), 3.40(\mathrm{dt}, J=6.3,1.6 \mathrm{~Hz}, 2 \mathrm{H})$, $2.69-2.59(\mathrm{~m}, 2 \mathrm{H}), 2.34(\mathrm{t}, J=7.4 \mathrm{~Hz}, 2 \mathrm{H}), 1.73(\mathrm{dt}, J=14.6,7.1 \mathrm{~Hz}, 2 \mathrm{H}), 1.62(\mathrm{p}, J=7.4 \mathrm{~Hz}, 2 \mathrm{H}), 1.26$ (t, $J=7.1 \mathrm{~Hz}, 3 \mathrm{H})$.

${ }^{13} \mathrm{C}$ NMR $\left(126 \mathrm{MHz}, \mathrm{CDCl}_{3}\right) \delta 173.72,140.37,137.62,137.44,129.75,129.29,126.49,126.22$, $115.76,60.36,37.16,34.37,32.52,30.56,25.15,14.39$.

HRMS(ESI): Calcd. for $\mathrm{C}_{16} \mathrm{H}_{22} \mathrm{O}_{2} \mathrm{Na}[\mathrm{M}+\mathrm{Na}]^{+}$269.1518. Found: 269.1509.

\section{6-(Thiazol-5-yl)-6-(trimethylsilyl)hexyl acetate (12)}

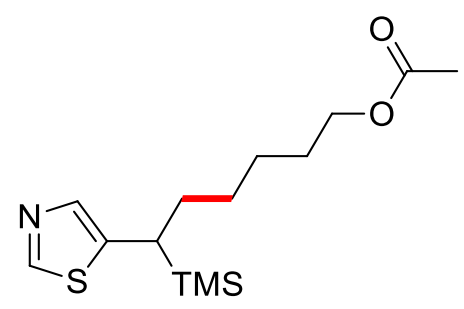

5-(1-(Trimethylsilyl)vinyl)thiazole $(36.7 \mathrm{mg}, 0.2 \mathrm{mmol}), 4-$ bromobutyl acetate $(117 \mathrm{mg}, 0.6$ mmol), $\mathrm{Fe}\left(\mathrm{NH}_{4}\right)_{2}\left(\mathrm{SO}_{4}\right)_{2} \cdot 6 \mathrm{H}_{2} \mathrm{O}(1.6 \mathrm{mg}, 0.004 \mathrm{mmol}), 3,4,7,8$-tetramethyl-1,10-phenanthroline (1 mg, $0.004 \mathrm{mmol}$ ), Zn dust (78 mg, $1.2 \mathrm{mmol})$ and NaOAc (8.2 mg, $0.1 \mathrm{mmol})$, in $0.4 \mathrm{~mL} 2 \mathrm{wt} \%$ TPGS-750$\mathrm{M} / \mathrm{H}_{2} \mathrm{O}$ with $\mathrm{MeCN}(0.04 \mathrm{~mL})$ as co-solvent were reacted at $\mathrm{rt}$ for $16 \mathrm{~h}$ yielding $55.5 \mathrm{mg}(93 \%)$ of 6(thiazol-5-yl)-6-(trimethylsilyl)hexyl acetate as a faint yellow oil (hexane/EtOAc: 80/20).

${ }^{1} \mathrm{H}$ NMR $\left(500 \mathrm{MHz}, \mathrm{CDCl}_{3}\right) \delta 8.56(\mathrm{~s}, 1 \mathrm{H}), 7.42(\mathrm{~s}, 1 \mathrm{H}), 3.99(\mathrm{t}, J=7 \mathrm{~Hz}, 2 \mathrm{H}), 2.32(\mathrm{dd}, J=12$ $\mathrm{Hz}, J=3 \mathrm{~Hz}, 1 \mathrm{H}), 2.01(\mathrm{~s}, 3 \mathrm{H}), 1.74(\mathrm{~m}, 1 \mathrm{H}), 1.55(\mathrm{~m}, 3 \mathrm{H}), 1.35(\mathrm{~m}, 2 \mathrm{H}), 1.23(\mathrm{~m}, 2 \mathrm{H}),-0.02(\mathrm{~s}, 9$ $\mathrm{H})$;

${ }^{13} \mathrm{C} \mathrm{NMR}\left(126 \mathrm{MHz}, \mathrm{CDCl}_{3}\right) \delta 171.25,149.90,142.18,138.99,64.56,31.69,28.97,28.87,28.52$, 25.76, 21.09, -3.02. 
HRMS(ESI): Calcd. for $\mathrm{C}_{14} \mathrm{H}_{26} \mathrm{NO}_{2} \mathrm{SSi}[\mathrm{M}+\mathrm{H}]^{+}$300.1454. Found: 300.1460 .

\section{Methyl 5-(5-ethoxy-5-oxopentyl)furan-2-carboxylate (13)}

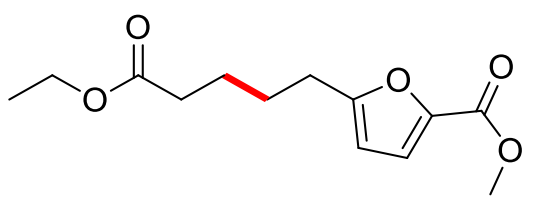

Methyl 5-vinylfuran-2-carboxylate (30.4 mg, $0.2 \mathrm{mmol}$ ), ethyl 3-bromopropanoate (108.6 mg, 0.6 mmol), Fe(acac) $)_{3}(1.4 \mathrm{mg}, 0.004 \mathrm{mmol}), 3,4,7,8$-tetramethyl-1,10-phenanthroline (1 mg, $\left.0.004 \mathrm{mmol}\right)$, $\mathrm{Zn}$ dust (78 mg, $1.2 \mathrm{mmol})$ and $\mathrm{NaCl}(70.2 \mathrm{mg}, 1.2 \mathrm{mmol})$, in $0.4 \mathrm{~mL} 2 \mathrm{wt} \% \mathrm{TPGS}-750-\mathrm{M} / \mathrm{H}_{2} \mathrm{O}$ were reacted at $\mathrm{rt}$ for $16 \mathrm{~h}$ yielding $36.7 \mathrm{mg}$ (72\%) of methyl 5-(5-ethoxy-5-oxopentyl)furan-2-carboxylate as a faint yellow oil (hexane/EtOAc: 90/10).

${ }^{1} \mathrm{H}$ NMR (500 MHz, CDCl $) \delta 7.05(\mathrm{~m}, 1 \mathrm{H}), 6.11(\mathrm{~m}, 1 \mathrm{H}), 4.08(\mathrm{q}, J=7.1 \mathrm{~Hz}, 2 \mathrm{H}), 3.83(\mathrm{~s}, 3 \mathrm{H})$, $2.68(\mathrm{t}, J=7.0 \mathrm{~Hz}, 2 \mathrm{H}), 2.29$ (t, $J=7.0 \mathrm{~Hz}, 2 \mathrm{H}), 1.73-1.60(\mathrm{~m}, 4 \mathrm{H}), 1.21(\mathrm{t}, J=7.1 \mathrm{~Hz}, 3 \mathrm{H})$.

${ }^{13} \mathrm{C}$ NMR $\left(126 \mathrm{MHz}, \mathrm{CDCl}_{3}\right) \delta 173.35,160.80,159.25,143.04,119.27,107.84,60.35,51.74$, $33.95,28.05,27.22,24.43,14.28$.

HRMS(ESI): Calcd. for $\mathrm{C}_{13} \mathrm{H}_{18} \mathrm{O}_{5} \mathrm{Na}[\mathrm{M}+\mathrm{Na}]^{+}$277.1052. Found: 277.1052 .

\section{Ethyl 5-(4-(1-methyl-1H-indazol-5-yl)thiophen-2-yl)pentanoate (14)}

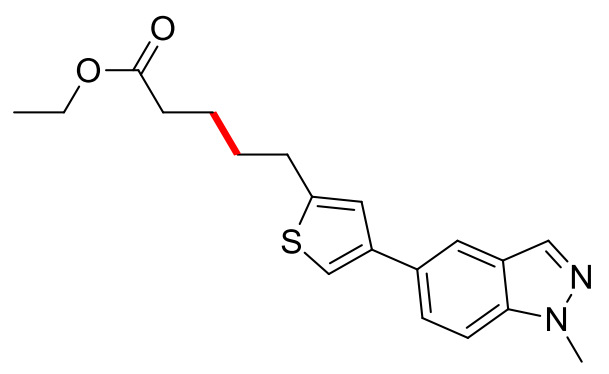

1-Methyl-5-(5-vinylthiophen-3-yl)-1H-indazole (24.0 mg, $0.1 \mathrm{mmol})$, ethyl 3-bromopropanoate (217.1 mg, $1.2 \mathrm{mmol}), \mathrm{Fe}(\mathrm{acac})_{3}(2.8 \mathrm{mg}, 0.008 \mathrm{mmol}), 3,4,7,8$-tetramethyl-1,10-phenanthroline ( $2 \mathrm{mg}$, $0.008 \mathrm{mmol}$ ), Zn dust (156 mg, $2.4 \mathrm{mmol}$ ), $0.1 \mathrm{~mL}$ THF and $\mathrm{NaCl}(140.4 \mathrm{mg}, 2.4 \mathrm{mmol})$, in $0.8 \mathrm{~mL} 2 \mathrm{wt} \%$ TPGS-750-M/ $\mathrm{H}_{2} \mathrm{O}$ were reacted at $45^{\circ} \mathrm{C}$ for $16 \mathrm{~h}$ yielding $19.6 \mathrm{mg}(57 \%)$ of ethyl 5-(4-(1-methyl- $1 \mathrm{H}-$ indazol-5-yl)thiophen-2-yl)pentanoate as a white solid (hexane/EtOAc: 80/20). 
${ }^{1} \mathrm{H}$ NMR (500 MHz, $\left.\mathrm{CDCl}_{3}\right) \delta 7.98(\mathrm{~s}, 1 \mathrm{H}), 7.88(\mathrm{~s}, 1 \mathrm{H}), 7.63(\mathrm{dd}, J=8.7,1.5 \mathrm{~Hz}, 1 \mathrm{H}), 7.39(\mathrm{~d}, J$ $=8.7 \mathrm{~Hz}, 1 \mathrm{H}), 7.21(\mathrm{~d}, J=1.4 \mathrm{~Hz}, 1 \mathrm{H}), 7.13(\mathrm{~s}, 1 \mathrm{H}), 4.13(\mathrm{q}, J=7.1 \mathrm{~Hz}, 2 \mathrm{H}), 4.08(\mathrm{~s}, 3 \mathrm{H}), 2.88(\mathrm{t}, J=$ $6.8 \mathrm{~Hz}, 2 \mathrm{H}), 2.36(\mathrm{t}, J=6.9 \mathrm{~Hz}, 2 \mathrm{H}), 1.82-1.72(\mathrm{~m}, 4 \mathrm{H}), 1.26(\mathrm{t}, J=7.1 \mathrm{~Hz}, 3 \mathrm{H})$.

${ }^{13} \mathrm{C}$ NMR $\left(126 \mathrm{MHz}, \mathrm{CDCl}_{3}\right) \delta 173.61,146.00,142.15,139.34,133.12,129.25,125.86,124.62$, $124.00,118.16,117.29,109.25,60.42,35.72,34.19,31.18,30.05,24.56,14.39$.

HRMS(ESI): Calcd. for $\mathrm{C}_{19} \mathrm{H}_{22} \mathrm{~N}_{2} \mathrm{O}_{2} \mathrm{SNa}$ [M+Na] $]^{+}$365.1300. Found: 365.1313 .

\section{6-(Thiazol-5-yl)hexyl acetate (15)}

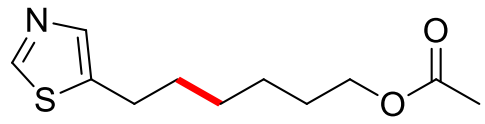

5-Vinylthiazole (22.3 mg, $0.2 \mathrm{mmol})$, 4-bromobutyl acetate $(117 \mathrm{mg}, 0.6 \mathrm{mmol})$, $\mathrm{Fe}\left(\mathrm{NH}_{4}\right)_{2}\left(\mathrm{SO}_{4}\right)_{2} \cdot 6 \mathrm{H}_{2} \mathrm{O}(1.6 \mathrm{mg}, 0.004 \mathrm{mmol}), 3,4,7,8$-tetramethyl-1,10-phenanthroline $(1 \mathrm{mg}, 0.004$ $\mathrm{mmol}$ ), $\mathrm{Zn}$ dust (78 mg, $1.2 \mathrm{mmol}$ ) and NaOAc ( $8.2 \mathrm{mg}, 0.1 \mathrm{mmol})$, in $0.4 \mathrm{~mL} 2 \mathrm{wt} \% \mathrm{TPGS}-750-\mathrm{M} / \mathrm{H}_{2} \mathrm{O}$ with $\mathrm{MeCN}(0.04 \mathrm{~mL})$ as co-solvent were reacted at $\mathrm{rt}$ for $16 \mathrm{~h}$ yielding $23.1 \mathrm{mg}(51 \%)$ of 6-(thiazol-5yl)hexyl acetate as a faint yellow liquid (hexane/EtOAc: 80/20).

${ }^{1} \mathrm{H}$ NMR (500 MHz, $\left.\mathrm{CDCl}_{3}\right) 8.64(\mathrm{~s}, 1 \mathrm{H}), 7.58(\mathrm{~s}, 1 \mathrm{H}), 4.04$ (t, $\left.J=6.7 \mathrm{~Hz}, 2 \mathrm{H}\right), 2.85$ (t, $J=7.6$ Hz, 2H), 2.04 (s, 3H), 1.69 (p, J=7.4 Hz, 2H), 1.61 (p, $J=6.8 \mathrm{~Hz}, 2 \mathrm{H}), 1.38$ (m, 4H).

${ }^{13} \mathrm{C}$ NMR $\left(126 \mathrm{MHz}, \mathrm{CDCl}_{3}\right) \delta 171.16,151.32,140.26,139.33,64.40,31.49,28.58,28.47,26.61$, 25.60, 20.99 .

HRMS(ESI): Calcd. for $\mathrm{C}_{11} \mathrm{H}_{17} \mathrm{NO}_{2} \mathrm{SNa}[\mathrm{M}+\mathrm{Na}]^{+} 250.0878$. Found: 250.0867

\section{Ethyl 5-(2-(piperidin-1-yl)pyrimidin-5-yl)pentanoate (16)}

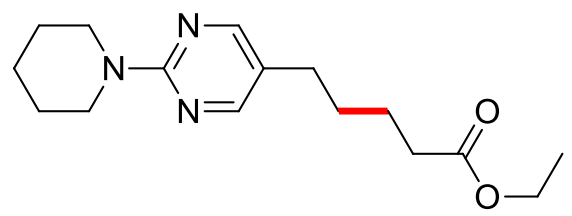

2-(Piperidin-1-yl)-5-vinylpyrimidine (18.9 mg, $0.1 \mathrm{mmol})$, ethyl 3-bromopropanoate (144.8 $\mathrm{mg}$, $0.8 \mathrm{mmol}$ ), Fe(acac) $)_{3}(2.8 \mathrm{mg}, 0.008 \mathrm{mmol}$ ), 3,4,7,8-tetramethyl-1,10-phenanthroline ( $2 \mathrm{mg}, 0.008 \mathrm{mmol}$ ), $\mathrm{Zn}$ dust (104 mg, $1.6 \mathrm{mmol}$ ) and $\mathrm{NaCl}(140.4 \mathrm{mg}, 2.4 \mathrm{mmol})$, in $0.8 \mathrm{~mL} 2 \mathrm{wt} \% \mathrm{TPGS}-750-\mathrm{M} / \mathrm{H}_{2} \mathrm{O}$ were 
reacted at $45^{\circ} \mathrm{C}$ for $16 \mathrm{~h}$ yielding $16.9 \mathrm{mg}$ (58\%) of ethyl 5-(2-(piperidin-1-yl)pyrimidin-5-yl)pentanoate as a faint yellow oil (hexane/EtOAc: 90/10).

${ }^{1} \mathrm{H}$ NMR $\left(500 \mathrm{MHz}, \mathrm{CDCl}_{3}\right) \delta 8.10(\mathrm{~s}, 2 \mathrm{H}), 4.09(\mathrm{q}, J=7.1 \mathrm{~Hz}, 2 \mathrm{H}), 3.74-3.68(\mathrm{~m}, 4 \mathrm{H}), 2.39(\mathrm{t}$, $J=7.4 \mathrm{~Hz}, 2 \mathrm{H}), 2.28(\mathrm{t}, J=7.3 \mathrm{~Hz}, 2 \mathrm{H}), 1.56(\mathrm{~m}, 10 \mathrm{H}), 1.21(\mathrm{t}, J=7.1 \mathrm{~Hz}, 3 \mathrm{H})$.

${ }^{13} \mathrm{C} \mathrm{NMR}\left(126 \mathrm{MHz}, \mathrm{CDCl}_{3}\right) \delta 173.49,161.02,157.50,121.75,60.34,45.02,34.15,30.81,29.25$, $25.80,24.98,24.37,14.34$.

HRMS(ESI): Calcd. for $\mathrm{C}_{16} \mathrm{H}_{25} \mathrm{~N}_{3} \mathrm{O}_{2} \mathrm{Na}[\mathrm{M}+\mathrm{Na}]^{+}$314.1844. Found: 314.1842

\section{t-Butyl 4-(5-ethoxy-5-oxopentyl)-1H-indole-1-carboxylate (17)}

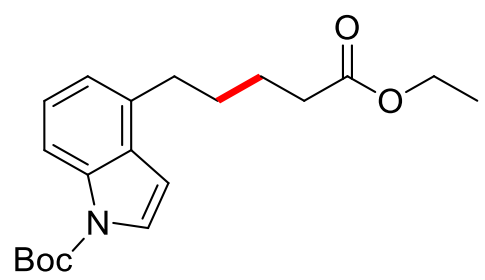

t-Butyl 4-vinyl-1H-indole-1-carboxylate $(24.3 \mathrm{mg}, 0.1 \mathrm{mmol})$, ethyl 3-bromopropanoate (217.2 $\mathrm{mg}, 1.2 \mathrm{mmol}), \mathrm{Fe}(\mathrm{acac})_{3}(2.8 \mathrm{mg}, 0.008 \mathrm{mmol}), 3,4,7,8$-tetramethyl-1,10-phenanthroline $(2 \mathrm{mg}, 0.008$ mmol), Zn dust (156 mg, $2.4 \mathrm{mmol}$ ) and $\mathrm{NaCl}$ (140.4 mg, $2.4 \mathrm{mmol}$ ), in $0.8 \mathrm{~mL} 2 \mathrm{wt} \% \mathrm{TPGS}-750-\mathrm{M} / \mathrm{H}_{2} \mathrm{O}$ were reacted at $\mathrm{rt}$ for $16 \mathrm{~h}$ yielding $19.3 \mathrm{mg}$ (56\%) of tert-butyl 4-(5-ethoxy-5-oxopentyl)-1H-indole-1carboxylate as a colorless oil (hexane/EtOAc: 90/10).

${ }^{1} \mathrm{H}$ NMR $\left(500 \mathrm{MHz}, \mathrm{CDCl}_{3}\right) \delta 8.00(\mathrm{~d}, J=7.9 \mathrm{~Hz}, 1 \mathrm{H}), 7.59(\mathrm{~d}, J=3.6 \mathrm{~Hz}, 1 \mathrm{H}), 7.26-7.21(\mathrm{~m}$, $1 \mathrm{H}), 7.04(\mathrm{~d}, J=7.3 \mathrm{~Hz}, 1 \mathrm{H}), 6.61(\mathrm{~d}, J=3.8 \mathrm{~Hz}, 1 \mathrm{H}), 4.12(\mathrm{q}, J=7.1 \mathrm{~Hz}, 2 \mathrm{H}), 2.88(\mathrm{t}, J=7.1 \mathrm{~Hz}, 2 \mathrm{H})$, $2.33(\mathrm{t}, J=7.0 \mathrm{~Hz}, 2 \mathrm{H}), 1.72(\mathrm{~m}, 4 \mathrm{H}), 1.67(\mathrm{~s}, 9 \mathrm{H}), 1.24(\mathrm{t}, J=7.1 \mathrm{~Hz}, 3 \mathrm{H})$.

${ }^{13} \mathrm{C}$ NMR $\left(126 \mathrm{MHz}, \mathrm{CDCl}_{3}\right) \delta 173.74,149.94,135.29,134.67,129.79,125.53,124.39,122.32$, $113.10,105.56,83.68,60.35,34.34,32.82,30.37,28.33,24.95,14.37$.

HRMS(ESI): Calcd. for $\mathrm{C}_{20} \mathrm{H}_{27} \mathrm{NO}_{4} \mathrm{Na}[\mathrm{M}+\mathrm{Na}]^{+}$368.1838. Found: 368.1829 


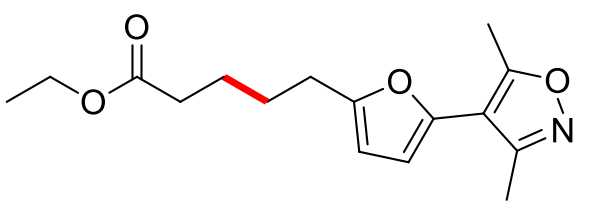

3,5-Dimethyl-4-(5-vinylfuran-2-yl)isoxazole $(37.8 \mathrm{mg}, 0.2 \mathrm{mmol})$, ethyl 3-bromopropanoate (108.6 mg, $0.6 \mathrm{mmol}), \mathrm{Fe}\left(\mathrm{NH}_{4}\right)_{2}\left(\mathrm{SO}_{4}\right)_{2} \cdot 6 \mathrm{H}_{2} \mathrm{O}(1.6 \mathrm{mg}, 0.004 \mathrm{mmol})$, 3,4,7,8-tetramethyl-1,10phenanthroline (1 mg, $0.004 \mathrm{mmol}), \mathrm{Zn}$ dust $(78 \mathrm{mg}, 1.2 \mathrm{mmol})$ and $\mathrm{NaOAc}(8.2 \mathrm{mg}, 0.1 \mathrm{mmol})$, in 0.4 $\mathrm{mL} 2 \mathrm{wt} \%$ TPGS-750-M/ $\mathrm{H}_{2} \mathrm{O}$ with $\mathrm{MeCN}(0.04 \mathrm{~mL})$ as co-solvent were reacted at $\mathrm{rt}$ for $16 \mathrm{~h}$ yielding $24.4 \mathrm{mg}(42 \%)$ of ethyl 5-(5-(3,5-dimethylisoxazol-4-yl)furan-2-yl)pentanoate as a faint yellow oil (hexane/EtOAc: 80/20).

${ }^{1} \mathrm{H}$ NMR (500 MHz, $\left.\mathrm{CDCl}_{3}\right) \delta 6.22(\mathrm{~d}, J=3.2 \mathrm{~Hz}, 1 \mathrm{H}), 6.07(\mathrm{~d}, J=4.0 \mathrm{~Hz}, 1 \mathrm{H}), 4.11(\mathrm{q}, J=7.1$ $\mathrm{Hz}, 2 \mathrm{H}), 2.68(\mathrm{t}, J=6.4 \mathrm{~Hz}, 2 \mathrm{H}), 2.54(\mathrm{~s}, 3 \mathrm{H}), 2.37$ (s, 3H), 2.33 (t, $J=6.9 \mathrm{~Hz}, 2 \mathrm{H}), 1.70(\mathrm{~m}, 4 \mathrm{H}), 1.24$ (t, $J=7.1 \mathrm{~Hz}, 3 \mathrm{H})$.

${ }^{13} \mathrm{C} \mathrm{NMR}\left(126 \mathrm{MHz}, \mathrm{CDCl}_{3}\right) \delta 173.55,164.90,157.74,155.36,143.87,108.58,107.38,106.58$, $60.40,34.13,27.78,27.59,24.54,14.36,12.49,11.67$.

HRMS(ESI): Calcd. for $\mathrm{C}_{16} \mathrm{H}_{21} \mathrm{NO}_{4} \mathrm{Na}[\mathrm{M}+\mathrm{Na}]^{+}$314.1368. Found: 314.1365

\section{Ethyl 5-(2-morpholinopyrimidin-5-yl)pentanoate (19)}

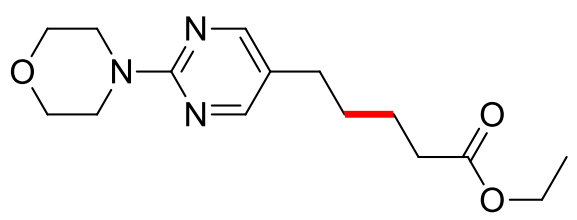

4-(5-Vinylpyrimidin-2-yl)morpholine (19.1 mg, $0.1 \mathrm{mmol})$, ethyl 3-bromopropanoate $(144.8 \mathrm{mg}$, $0.8 \mathrm{mmol}$ ), Fe(acac) $)_{3}(2.8 \mathrm{mg}, 0.008 \mathrm{mmol}), 3,4,7,8$-tetramethyl-1,10-phenanthroline ( $\left.2 \mathrm{mg}, 0.008 \mathrm{mmol}\right)$, $\mathrm{Zn}$ dust (104 mg, $1.6 \mathrm{mmol}$ ) and $\mathrm{NaCl}(140.4 \mathrm{mg}, 2.4 \mathrm{mmol})$, in $0.8 \mathrm{~mL} 2 \mathrm{wt} \% \mathrm{TPGS}-750-\mathrm{M} / \mathrm{H}_{2} \mathrm{O}$ were reacted at $45^{\circ} \mathrm{C}$ for $16 \mathrm{~h}$ yielding $16.4 \mathrm{mg}(56 \%)$ of ethyl 5-(2-morpholinopyrimidin-5-yl)pentanoate as a colorless oil (hexane/EtOAc: 80/20).

${ }^{1} \mathrm{H}$ NMR (500 MHz, $\left.\mathrm{CDCl}_{3}\right) \delta 8.14(\mathrm{~s}, 2 \mathrm{H}), 4.09(\mathrm{q}, J=7.1 \mathrm{~Hz}, 2 \mathrm{H}), 3.73(\mathrm{~s}, 8 \mathrm{H}), 2.42(\mathrm{t}, J=7.4$ $\mathrm{Hz}, 2 \mathrm{H}), 2.29$ (t, $J=7.3 \mathrm{~Hz}, 2 \mathrm{H}), 1.62$ (q, $J=7.9,7.1 \mathrm{~Hz}, 2 \mathrm{H}), 1.56$ (q, $J=7.3 \mathrm{~Hz}, 2 \mathrm{H}), 1.22$ (t, $J=7.1$ $\mathrm{Hz}, 3 \mathrm{H})$. 
${ }^{13} \mathrm{C}$ NMR $\left(126 \mathrm{MHz}, \mathrm{CDCl}_{3}\right) \delta 173.44,161.04,157.52,123.13,66.91,60.36,44.51,34.10,30.73$, $29.26,24.35,14.34$.

HRMS(ESI): Calcd. for $\mathrm{C}_{15} \mathrm{H}_{23} \mathrm{~N}_{3} \mathrm{O}_{3} \mathrm{Na}[\mathrm{M}+\mathrm{Na}]^{+}$316.1637. Found: 316.1633

Ethyl 5-(1H-indol-5-yl)pentanoate (20)

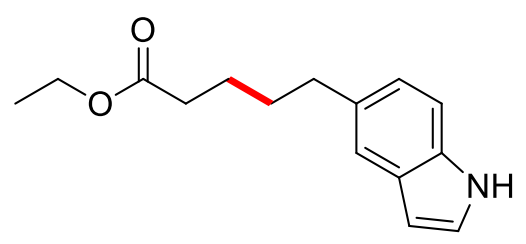

5-Vinyl-1H-indole (14.3 mg, $0.1 \mathrm{mmol})$, ethyl 3-bromopropanoate (144.8 mg, $0.8 \mathrm{mmol})$, $\mathrm{Fe}(\mathrm{acac})_{3}(2.8 \mathrm{mg}, 0.008 \mathrm{mmol}), 3,4,7,8$-tetramethyl-1,10-phenanthroline (2 $\left.\mathrm{mg}, 0.008 \mathrm{mmol}\right), \mathrm{Zn}$ dust (156 mg, $2.4 \mathrm{mmol}$ ) and $\mathrm{NaCl}$ (140.4 mg, $2.4 \mathrm{mmol}$ ), in $0.8 \mathrm{~mL} 2 \mathrm{wt} \% \mathrm{TPGS}-750-\mathrm{M} / \mathrm{H}_{2} \mathrm{O}$ were reacted at $\mathrm{rt}$ for $16 \mathrm{~h}$ yielding $17.9 \mathrm{mg}$ (73\%) of ethyl 5-(1H-indol-5-yl)pentanoate as a colorless oil (hexane/EtOAc: 90/10).

${ }^{1} \mathrm{H}$ NMR (500 MHz, $\left.\mathrm{CDCl}_{3}\right) \delta 8.14(\mathrm{~s}, 1 \mathrm{H}), 7.45(\mathrm{~s}, 1 \mathrm{H}), 7.30(\mathrm{~d}, J=8.3 \mathrm{~Hz}, 1 \mathrm{H}), 7.19-7.15(\mathrm{~m}$, $1 \mathrm{H}), 7.04(\mathrm{dd}, J=8.3,1.5 \mathrm{~Hz}, 1 \mathrm{H}), 6.50(\mathrm{~m}, 1 \mathrm{H}), 4.14(\mathrm{q}, J=7.1 \mathrm{~Hz}, 2 \mathrm{H}), 2.74(\mathrm{t}, J=6.9 \mathrm{~Hz}, 2 \mathrm{H}), 2.35$ (t, $J=7.1 \mathrm{~Hz}, 2 \mathrm{H}), 1.72(\mathrm{~m}, 4 \mathrm{H}), 1.26(\mathrm{t}, J=7.1 \mathrm{~Hz}, 3 \mathrm{H})$.

${ }^{13} \mathrm{C}$ NMR $\left(126 \mathrm{MHz}, \mathrm{CDCl}_{3}\right) \delta 173.98,134.49,133.62,128.17,124.41,123.05,119.91,110.88$, $102.29,60.33,35.76,34.45,31.80,24.80,14.37$.

HRMS(ESI): Calcd. for $\mathrm{C}_{15} \mathrm{H}_{19} \mathrm{NO}_{2} \mathrm{Na}[\mathrm{M}+\mathrm{Na}]^{+} 268.1313$. Found: 268.1325

\section{4-(5-(4-(3-Bromophenyl)piperazin-1-yl)-5-oxopentyl)-N-(3,5-dimethylisoxazol-4-yl)benzamide (21)}<smiles>Cc1noc(C)c1NC(=O)c1ccc(CCCCC(=O)N2CCN(c3cccc(Br)c3)CC2)cc1</smiles>

$\mathrm{N}$-(3,5-dimethylisoxazol-4-yl)-4-vinylbenzamide (48.4 mg, $0.2 \mathrm{mmol}), \quad 3-$-bromo-1-(4-(3bromophenyl)piperazin-1-yl)propan-1-one (225.7 mg, $0.6 \mathrm{mmol}), \mathrm{Fe}\left(\mathrm{NH}_{4}\right)_{2}\left(\mathrm{SO}_{4}\right)_{2} \cdot 6 \mathrm{H}_{2} \mathrm{O}(8 \mathrm{mg}, 0.02$ mmol), 3,4,7,8-tetramethyl-1,10-phenanthroline (5 mg, $0.02 \mathrm{mmol})$, Zn dust $(78 \mathrm{mg}, 1.2 \mathrm{mmol})$ and 
NaOAc (8.2 mg, $0.1 \mathrm{mmol})$, in $0.4 \mathrm{~mL} 2$ wt \% TPGS-750-M/ $\mathrm{H}_{2} \mathrm{O}$ with $\mathrm{MeCN}(0.04 \mathrm{~mL})$ as co-solvent were reacted at $45{ }^{\circ} \mathrm{C}$ for $16 \mathrm{~h}$ yielding $56.2 \mathrm{mg}$ (52\%) of 4-(5-(4-(3-bromophenyl)piperazin-1-yl)-5oxopentyl)-N-(3,5-dimethylisoxazol-4-yl)benzamide as a white solid (hexane/EtOAc: 25/75); mp 164 $166{ }^{\circ} \mathrm{C}$.

${ }^{1} \mathrm{H}$ NMR (400 MHz, $\left.\mathrm{CDCl}_{3}\right) \delta 9.17(\mathrm{~s}, 1 \mathrm{H}), 7.84(\mathrm{~d}, J=7.7 \mathrm{~Hz}, 2 \mathrm{H}), 7.23(\mathrm{~d}, J=7.6 \mathrm{~Hz}, 2 \mathrm{H}), 7.10$ $(\mathrm{t}, J=7.9 \mathrm{~Hz}, 1 \mathrm{H}), 6.98(\mathrm{~d}, J=8.4 \mathrm{~Hz}, 2 \mathrm{H}), 6.79(\mathrm{~d}, J=7.8 \mathrm{~Hz}, 1 \mathrm{H}), 3.78-3.64(\mathrm{~m}, 2 \mathrm{H}), 3.62-3.49(\mathrm{~m}$, 2H), $3.21-2.99(\mathrm{~m}, 4 \mathrm{H}), 2.67(\mathrm{t}, J=7.5 \mathrm{~Hz}, 2 \mathrm{H}), 2.36(\mathrm{t}, J=7.5 \mathrm{~Hz}, 2 \mathrm{H}), 2.17(\mathrm{~s}, 3 \mathrm{H}), 1.90(\mathrm{~s}, 3 \mathrm{H}), 1.75$ $-1.54(\mathrm{~m}, 4 \mathrm{H})$.

${ }^{13} \mathrm{C} \mathrm{NMR}\left(126 \mathrm{MHz}, \mathrm{CDCl}_{3}\right) \delta 171.60,164.81,162.03,156.79,152.10,147.38,130.54,130.39$, $128.79,128.07,123.32,123.11,119.26,114.96,103.75,49.18,48.88,45.35,41.40,35.69,33.00,30.73$, $24.73,10.76,7.46$.

HRMS(ESI): Calcd. for $\mathrm{C}_{27} \mathrm{H}_{31} \mathrm{BrN}_{4} \mathrm{O}_{3} \mathrm{Na}[\mathrm{M}+\mathrm{Na}]^{+}$561.1477. Found: 561.1473

\section{2-(5-Phenoxypentyl)pyridine (22)}

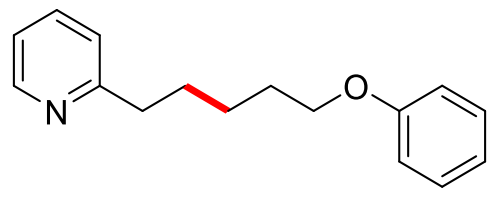

2-Vinylpyridine (21 mg, $0.2 \mathrm{mmol}$ ), (3-bromopropoxy)benzene (129.1 mg, $0.6 \mathrm{mmol}$ ), $\mathrm{Fe}\left(\mathrm{NH}_{4}\right)_{2}\left(\mathrm{SO}_{4}\right)_{2} \cdot 6 \mathrm{H}_{2} \mathrm{O}(1.6 \mathrm{mg}, 0.004 \mathrm{mmol}), 3,4,7,8$-tetramethyl-1,10-phenanthroline (1 mg, 0.004 mmol), Zn dust (78 mg, $1.2 \mathrm{mmol}$ ) and NaOAc (8.2 mg, $0.1 \mathrm{mmol})$, in $0.4 \mathrm{~mL} 2 \mathrm{wt} \%$ TPGS-750-M/ $\mathrm{H}_{2} \mathrm{O}$ with $\mathrm{MeCN}(0.04 \mathrm{~mL})$ as co-solvent were reacted at $\mathrm{rt}$ for $16 \mathrm{~h}$ yielding $35.5 \mathrm{mg}(74 \%)$ of 2-(5phenoxypentyl)pyridine as a slight yellow oil (hexane/EtOAc: 80/20).

${ }^{1} \mathrm{H}$ NMR (500 MHz, $\left.\mathrm{CDCl}_{3}\right) \delta 8.52(\mathrm{~d}, J=4.2 \mathrm{~Hz}, 1 \mathrm{H}), 7.61-7.55(\mathrm{~m}, 1 \mathrm{H}), 7.30-7.23(\mathrm{~m}, 2 \mathrm{H})$, 7.17-7.06 (m, 2H), 6.95-6.85 (m, 3H), $3.95(\mathrm{t}, J=6.5 \mathrm{~Hz}, 2 \mathrm{H}), 2.83(\mathrm{t}, J=8.0 \mathrm{~Hz}, 2 \mathrm{H}), 1.88-1.77(\mathrm{~m}, 4 \mathrm{H})$, $1.59-1.50(\mathrm{~m}, 2 \mathrm{H})$.

${ }^{13} \mathrm{C} \mathrm{NMR}\left(126 \mathrm{MHz}, \mathrm{CDCl}_{3}\right) \delta 162.2,159.2,149.3,136.5,129.5,122.9,121.1,120.6,114.6,67.8$, $38.4,29.7,29.3,26.0$.

HRMS(EI): Calcd. for $\mathrm{C}_{16} \mathrm{H}_{19} \mathrm{NO}[\mathrm{M}]^{+}$241.1467. Found: 241.1461. 


\section{2-(4-Phenylbutyl)pyridine (23)}

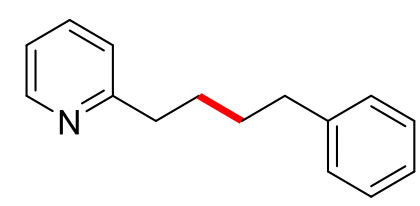

2-Vinylpyridine (21 mg, $0.2 \mathrm{mmol})$, (2-bromoethyl)benzene (111 mg, $0.6 \mathrm{mmol}), \mathrm{Fe}(\mathrm{acac})_{3}(1.4$ mg, $0.004 \mathrm{mmol})$, 3,4,7,8-tetramethyl-1,10-phenanthroline (1 mg, $0.004 \mathrm{mmol})$, Zn dust (78 mg, 1.2 $\mathrm{mmol}$ ) and $\mathrm{NaCl}(70.2 \mathrm{mg}, 1.2 \mathrm{mmol})$, in $0.4 \mathrm{~mL} 2 \mathrm{wt} \%$ TPGS-750-M/ $\mathrm{H}_{2} \mathrm{O}$ were reacted at $\mathrm{rt}$ for $16 \mathrm{~h}$ yielding $34.1 \mathrm{mg}(81 \%)$ of 2-(4-phenylbutyl)pyridine as a slight yellow oil (hexane/EtOAc: 80/20).

${ }^{1} \mathrm{H}$ NMR $\left(500 \mathrm{MHz}, \mathrm{CDCl}_{3}\right) \delta$ 8.60-8.47 (m, 1H), 7.63-7.54 (m, 1H), 7.31-7.23 (m, 2H), 7.22$7.05(\mathrm{~m}, 5 \mathrm{H}), 2.82(\mathrm{t}, J=7.5 \mathrm{~Hz}, 2 \mathrm{H}), 2.65(\mathrm{t}, J=7.5 \mathrm{~Hz}, 2 \mathrm{H}), 1.85-1.75(\mathrm{~m}, 2 \mathrm{H}), 1.74-1.65(\mathrm{~m}, 2 \mathrm{H})$.

${ }^{13} \mathrm{C} \mathrm{NMR}\left(126 \mathrm{MHz} \mathrm{CDCl}_{3}\right) \delta 162.2,149.2,142.6,136.5,128.5,128.4,125.8,122.9,121.1,38.3$, $35.9,31.3,29.6$.

HRMS(EI): Calcd. for $\mathrm{C}_{15} \mathrm{H}_{17} \mathrm{~N}[\mathrm{M}]^{+} 211.1361$. Found: 211.1351.

\section{Ethyl 5-(pyridin-2-yl)pentanoate (24)}

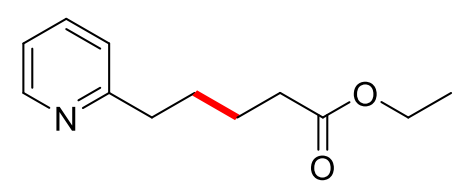

2-Vinylpyridine (21 mg, $0.2 \mathrm{mmol}$ ), ethyl 3-bromopropanoate (108.6 mg, $0.6 \mathrm{mmol}$ ), $\mathrm{Fe}\left(\mathrm{NH}_{4}\right)_{2}\left(\mathrm{SO}_{4}\right)_{2} \cdot 6 \mathrm{H}_{2} \mathrm{O}(1.6 \mathrm{mg}, 0.004 \mathrm{mmol}), 3,4,7,8$-tetramethyl-1,10-phenanthroline (1 mg, 0.004 mmol), Zn dust (78 mg, $1.2 \mathrm{mmol}$ ) and NaOAc (8.2 mg, $0.1 \mathrm{mmol})$, in $0.4 \mathrm{~mL} 2 \mathrm{wt} \%$ TPGS-750-M/ $\mathrm{H}_{2} \mathrm{O}$ with $\mathrm{MeCN}(0.04 \mathrm{~mL})$ as co-solvent were reacted at $\mathrm{rt}$ for $16 \mathrm{~h}$ yielding $29.1 \mathrm{mg}$ (70\%) of ethyl 5-(pyridin2-yl)pentanoate as a slight yellow oil (hexane/EtOAc: 70/30).

${ }^{1} \mathrm{H}$ NMR $\left(500 \mathrm{MHz}, \mathrm{CDCl}_{3}\right) \delta 8.51(\mathrm{~d}, J=4.8 \mathrm{~Hz}, 1 \mathrm{H}), 7.61-7.55(\mathrm{~m}, 1 \mathrm{H}), 7.13(\mathrm{~d}, J=7.8 \mathrm{~Hz}$, $1 \mathrm{H}), 7.11-7.06(\mathrm{~m}, 1 \mathrm{H}), 4.10(\mathrm{q}, J=7.1 \mathrm{~Hz}, 2 \mathrm{H}), 2.80(\mathrm{t}, J=7.6 \mathrm{~Hz}, 2 \mathrm{H}), 2.32(\mathrm{t}, J=7.4 \mathrm{~Hz}, 2 \mathrm{H}), 1.82-$ $1.72(\mathrm{~m}, 2 \mathrm{H}), 1.72-1.63(\mathrm{~m}, 2 \mathrm{H}), 1.23(\mathrm{t}, J=7.1 \mathrm{~Hz}, 3 \mathrm{H})$. 
${ }^{13} \mathrm{C}$ NMR $\left(126 \mathrm{MHz}, \mathrm{CDCl}_{3}\right) \delta 173.7,161.8,149.2,136.6,122.9,121.2,60.4,38.0,34.3,29.3$, $24.8,14.4$.

HRMS(ESI): Calcd. for $\mathrm{C}_{12} \mathrm{H}_{18} \mathrm{NO}_{2}[\mathrm{M}+\mathrm{H}]^{+}$208.1338. Found: 208.1344.

\section{2-(Hex-5-en-1-yl)pyridine (25)}

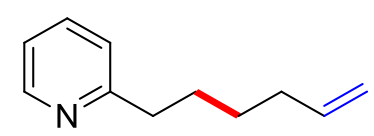

2-Vinylpyridine (21 mg, $0.2 \mathrm{mmol}$ ), 4-bromo-1-butene (81 mg, $0.6 \mathrm{mmol}), \mathrm{Fe}\left(\mathrm{NH}_{4}\right)_{2}\left(\mathrm{SO}_{4}\right)_{2} \cdot 6 \mathrm{H}_{2} \mathrm{O}$ (1.6 mg, $0.004 \mathrm{mmol}$ ), 3,4,7,8-tetramethyl-1,10-phenanthroline (1 mg, $0.004 \mathrm{mmol}$ ), Zn dust (78 mg, 1.2 mmol) and $\mathrm{NaOAc}(8.2 \mathrm{mg}, 0.1 \mathrm{mmol})$, in $0.4 \mathrm{~mL} 2 \mathrm{wt} \%$ TPGS-750-M/ $\mathrm{H}_{2} \mathrm{O}$ with $\mathrm{MeCN}(0.04 \mathrm{~mL})$ as co-solvent were reacted at $\mathrm{rt}$ for $16 \mathrm{~h}$ yielding $21.8 \mathrm{mg}(68 \%)$ of 2-(hex-5-en-1-yl)pyridine as a slight yellow oil (hexane/EtOAc: 80/20).

${ }^{1} \mathrm{H}$ NMR $\left(500 \mathrm{MHz}, \mathrm{CDCl}_{3}\right) \delta$ 8.57-8.47 (m, 1H), 7.63-7.52 (m, 1H), $7.14(\mathrm{~d}, J=7.8 \mathrm{~Hz}, 1 \mathrm{H})$, 7.11-7.05 (m, 1H), 5.87-5.73 (m, 1H), 5.03-4.96 (m, 1H), 4.95-4.89 (m, 1H), $2.79(\mathrm{t}, J=8.0 \mathrm{~Hz}, 2 \mathrm{H})$, 2.14-2.02 (m, 2H), 1.80-1.69 (m, 2H), 1.52-1.39 (m, 2H).

${ }^{13} \mathrm{C}$ NMR $\left(126 \mathrm{MHz}, \mathrm{CDCl}_{3}\right) \delta 162.4,149.2,138.9,136.5,122.9,121.1,114.6,38.3,33.8,29.5$, 28.8 .

HRMS(EI): Calcd. for $\mathrm{C}_{11} \mathrm{H}_{15} \mathrm{~N}[\mathrm{M}]^{+}$161.1205. Found: 161.1198.

\section{(9H-Fluoren-9-yl)methyl 5-(pyridin-2-yl)pentanoate (26)}

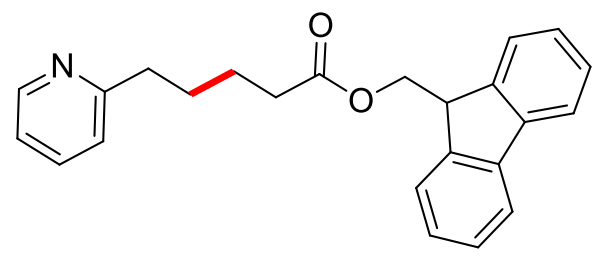

2-Vinylpyridine (21 mg, $0.2 \mathrm{mmol}$ ), (9H-fluoren-9-yl)methyl 3-bromopropanoate (198.7 mg, 0.6 mmol), $\mathrm{Fe}\left(\mathrm{NH}_{4}\right)_{2}\left(\mathrm{SO}_{4}\right)_{2} \cdot 6 \mathrm{H}_{2} \mathrm{O}$ (1.6 mg, $\left.0.004 \mathrm{mmol}\right)$, 3,4,7,8-tetramethyl-1,10-phenanthroline (1 mg, $0.004 \mathrm{mmol}$ ), Zn dust (78 mg, $1.2 \mathrm{mmol})$ and NaOAc (8.2 mg, $0.1 \mathrm{mmol})$, in $0.4 \mathrm{~mL} 2 \mathrm{wt} \%$ TPGS-750$\mathrm{M} / \mathrm{H}_{2} \mathrm{O}$ with $\mathrm{MeCN}(0.04 \mathrm{~mL})$ as co-solvent were reacted at $\mathrm{rt}$ for $16 \mathrm{~h}$ yielding $54.3 \mathrm{mg}(76 \%)$ of $(9 \mathrm{H}-$ 
fluoren-9-yl)methyl 5-(pyridin-2-yl)pentanoate as a faint viscous yellow oil (hexane/EtOAc: 80/20).

${ }^{1} \mathrm{H}$ NMR (500 MHz, $\left.\mathrm{CDCl}_{3}\right) \delta 8.54(\mathrm{~d}, J=4 \mathrm{~Hz}, 1 \mathrm{H}), 7.76(\mathrm{~d}, J=7.5 \mathrm{~Hz}, 2 \mathrm{H}), 7.57(\mathrm{~m}, 3 \mathrm{H})$, 7.39 (m, 2 H), 7.30 (m, 2 H), 7.12 (m, 2 H), 4.38 (d, $J=7$ Hz, 2 H), 4.19 (t, $J=7$ Hz, 1 H), 2.81 (t, $J=7$ $\mathrm{Hz}, 2 \mathrm{H}), 2.43(\mathrm{t}, J=7 \mathrm{~Hz}, 2 \mathrm{H}), 1.72(\mathrm{~m}, 4 \mathrm{H})$;

${ }^{13} \mathrm{C}$ NMR (126 MHz, $\left.\mathrm{CDCl}_{3}\right) \delta 173.50,161.73,149.30,143.90,141.36,136.38,127.81,127.14$, $125.07,122.82,121.11,120.07,66.23,46.93,37.97,34.24,29.20,24.65$.

HRMS(ESI): Calcd. for $\mathrm{C}_{24} \mathrm{H}_{24} \mathrm{NO}_{2}[\mathrm{M}+\mathrm{H}]^{+}$358.1807. Found: $358.1801 ; \mathrm{C}_{24} \mathrm{H}_{23} \mathrm{NO}_{2} \mathrm{Na}[\mathrm{M}+\mathrm{Na}]^{+}$ 380.1627. Found: 380.1621.

\section{$N$-(4-Chloro-3-(trifluoromethyl)phenyl)-5-(pyridin-2-yl)pentanamide (27)}

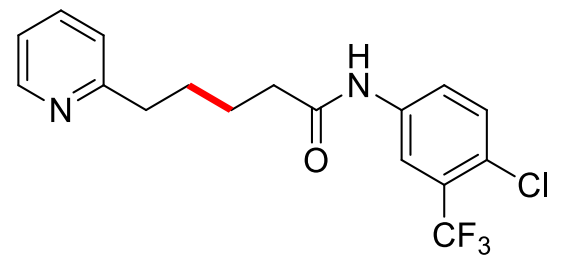

2-Vinylpyridine (21 $\mathrm{mg}, \quad 0.2 \mathrm{mmol}), \quad 3$-bromo- $N$-(4-chloro-3-(trifluoromethyl)phenyl)propanamide (198.3 mg, $0.6 \mathrm{mmol}), \mathrm{Fe}\left(\mathrm{NH}_{4}\right)_{2}\left(\mathrm{SO}_{4}\right)_{2} \cdot 6 \mathrm{H}_{2} \mathrm{O}$ (1.6 mg, $\left.0.004 \mathrm{mmol}\right), 3,4,7,8-$ tetramethyl-1,10-phenanthroline (1 mg, $0.004 \mathrm{mmol}), \mathrm{Zn}$ dust $(78 \mathrm{mg}, 1.2 \mathrm{mmol})$ and $\mathrm{NaOAc}(8.2 \mathrm{mg}$, $0.1 \mathrm{mmol})$, in $0.4 \mathrm{~mL} 2 \mathrm{wt} \%$ TPGS-750-M/ $\mathrm{H}_{2} \mathrm{O}$ with $\mathrm{MeCN}(0.04 \mathrm{~mL})$ as co-solvent were reacted at $\mathrm{rt}$ for $16 \mathrm{~h}$ yielding $53.6 \mathrm{mg}$ (75\%) of $N$-(4-chloro-3-(trifluoromethyl)phenyl)-5-(pyridin-2-yl)pentanamide as a faint yellow oil (hexane/EtOAc: 80/20).

${ }^{1} \mathrm{H} \mathrm{NMR}\left(500 \mathrm{MHz}, \mathrm{CDCl}_{3}\right) \delta 8.64(\mathrm{~s}, 1 \mathrm{H}), 8.48(\mathrm{~d}, J=5 \mathrm{~Hz}, 1 \mathrm{H}), 7.87(\mathrm{~m}, 1 \mathrm{H}), 7.76(\mathrm{~m}, 1 \mathrm{H})$, $7.61(\mathrm{~m}, 1 \mathrm{H}), 7.40(\mathrm{~d}, J=9 \mathrm{~Hz}, 1 \mathrm{H}), 7.17(\mathrm{~d}, J=8 \mathrm{~Hz}, 1 \mathrm{H}), 7.13(\mathrm{~m}, 1 \mathrm{H}), 2.84$ (t, $J=7 \mathrm{~Hz}, 2 \mathrm{H}), 2.41$ (t, $J=7 \mathrm{~Hz}, 2 \mathrm{H}), 1.79(\mathrm{~m}, 4 \mathrm{H})$;

${ }^{13} \mathrm{C} \mathrm{NMR}\left(126 \mathrm{MHz} \mathrm{CDCl}_{3}\right) \delta 172.0,161.6,149.0,137.4,136.9,132.0,128.9\left(\mathrm{q},{ }^{1} J_{(\mathrm{C}-\mathrm{F})}=297\right.$ $\mathrm{Hz}), 128.8\left(\mathrm{q},{ }^{1} J_{(\mathrm{C}-\mathrm{F})}=32 \mathrm{~Hz}\right), 124.0,123.8\left(\mathrm{q},{ }^{1} J_{(\mathrm{C}-\mathrm{F})}=272 \mathrm{~Hz}\right), 123.4,121.5,119.0\left(\mathrm{q},{ }^{1} J_{(\mathrm{C}-\mathrm{F})}=5.6 \mathrm{~Hz}\right)$, $37.4,37.1,28.8,24.8$;

${ }^{19}$ F NMR $\left(376 \mathrm{MHz}, \mathrm{CDCl}_{3}\right) \delta-62.83$

HRMS(ESI): Calcd. for $\mathrm{C}_{17} \mathrm{H}_{17} \mathrm{ClF}_{3} \mathrm{~N}_{2} \mathrm{O}[\mathrm{M}+\mathrm{H}]^{+}$357.0981. Found: 357.0977. 


\section{4-Chlorobenzyl 5-(pyridin-2-yl)pentanoate (28)}

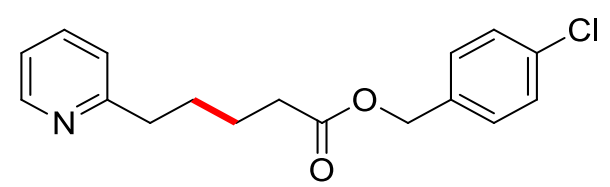

2-Vinylpyridine (21 mg, $0.2 \mathrm{mmol})$, 4-chlorobenzyl 3-bromopropanoate (166.5 mg, $0.6 \mathrm{mmol})$, $\mathrm{Fe}\left(\mathrm{NH}_{4}\right)_{2}\left(\mathrm{SO}_{4}\right)_{2} \cdot 6 \mathrm{H}_{2} \mathrm{O}(1.6 \mathrm{mg}, 0.004 \mathrm{mmol}), 3,4,7,8$-tetramethyl-1,10-phenanthroline (1 mg, 0.004 mmol), Zn dust (78 mg, $1.2 \mathrm{mmol}$ ) and NaOAc ( $8.2 \mathrm{mg}, 0.1 \mathrm{mmol})$, in $0.4 \mathrm{~mL} 2 \mathrm{wt} \% \mathrm{TPGS}-750-\mathrm{M} / \mathrm{H}_{2} \mathrm{O}$ with $\mathrm{MeCN}(0.04 \mathrm{~mL})$ as co-solvent were reacted at $\mathrm{rt}$ for $16 \mathrm{~h}$ yielding $48.6 \mathrm{mg}(80 \%)$ of 4-chlorobenzyl 5-(pyridin-2-yl)pentanoate as a faint yellow oil (hexane/EtOAc: 80/20).

${ }^{1} \mathrm{H}$ NMR $\left(500 \mathrm{MHz}, \mathrm{CDCl}_{3}\right) \delta 8.50(\mathrm{~d}, J=4 \mathrm{~Hz}, 1 \mathrm{H}), 7.55(\mathrm{~m}, 1 \mathrm{H}), 7.29(\mathrm{~m}, 2 \mathrm{H}), 7.26(\mathrm{~m}, 2 \mathrm{H})$, $7.08(\mathrm{~m}, 2 \mathrm{H}), 5.04(\mathrm{~s}, 2 \mathrm{H}), 2.77$ (t, $J=7.5 \mathrm{~Hz}, 2 \mathrm{H}), 2.38(\mathrm{t}, J=7.5 \mathrm{~Hz}, 2 \mathrm{H}), 1.71(\mathrm{~m}, 4 \mathrm{H})$;

${ }^{13} \mathrm{C} \mathrm{NMR}\left(126 \mathrm{MHz}, \mathrm{CDCl}_{3}\right) \delta 173.3,161.7,149.3,136.4,134.7,134.1,129.7,128.8,122.8,121.1$, $65.4,38.0,34.2,29.2,24.7$.

HRMS(ESI): Calcd. for $\mathrm{C}_{17} \mathrm{H}_{18} \mathrm{ClNO}_{2} \mathrm{Na}[\mathrm{M}+\mathrm{Na}]^{+}$326.0924. Found: 326.0916 .

\section{6-(Pyridin-2-yl)hexyl acetate (29)}

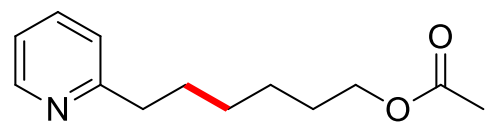

2-Vinylpyridine (21 mg, $0.2 \mathrm{mmol})$, 4-bromobutyl acetate $(117 \mathrm{mg}, 0.6 \mathrm{mmol})$, $\mathrm{Fe}\left(\mathrm{NH}_{4}\right)_{2}\left(\mathrm{SO}_{4}\right)_{2} \cdot 6 \mathrm{H}_{2} \mathrm{O}(1.6 \mathrm{mg}, 0.004 \mathrm{mmol}), 3,4,7,8$-tetramethyl-1,10-phenanthroline (1 mg, 0.004 mmol), Zn dust (78 mg, $1.2 \mathrm{mmol}$ ) and NaOAc ( $8.2 \mathrm{mg}, 0.1 \mathrm{mmol})$, in $0.4 \mathrm{~mL} 2 \mathrm{wt} \% \mathrm{TPGS}-750-\mathrm{M} / \mathrm{H}_{2} \mathrm{O}$ with $\mathrm{MeCN}(0.04 \mathrm{~mL})$ as co-solvent were reacted at $45^{\circ} \mathrm{C}$ for $16 \mathrm{~h}$ yielding $27.4 \mathrm{mg}(62 \%)$ of 6-(pyridin2-yl)hexyl acetate as a faint yellow oil (hexane/EtOAc: 80/20).

${ }^{1} \mathrm{H}$ NMR $\left(500 \mathrm{MHz}, \mathrm{CDCl}_{3}\right) \delta 8.50(\mathrm{~d}, J=4 \mathrm{~Hz}, 1 \mathrm{H}), 7.56(\mathrm{~m}, 1 \mathrm{H}), 7.12(\mathrm{~d}, J=8 \mathrm{~Hz}, 1 \mathrm{H}), 7.07$ $(\mathrm{m}, 1 \mathrm{H}), 4.02(\mathrm{t}, J=4 \mathrm{~Hz}, 2 \mathrm{H}), 2.76(\mathrm{t}, J=4 \mathrm{~Hz}, 2 \mathrm{H}), 2.01(\mathrm{~s}, 3 \mathrm{H}), 1.72(\mathrm{~m}, 2 \mathrm{H}), 1.60(\mathrm{~m}, 2 \mathrm{H}), 1.37$ $(\mathrm{m}, 4 \mathrm{H})$;

${ }^{13} \mathrm{C}$ NMR $\left(126 \mathrm{MHz}, \mathrm{CDCl}_{3}\right) \delta 171.29,162.32,149.28,136.36,122.78,121.01,64.63,38.37$, 
$29.81,29.05,28.60,25.88,21.09$.

HRMS(ESI): Calcd. for $\mathrm{C}_{13} \mathrm{H}_{19} \mathrm{NO}_{2} \mathrm{Na}[\mathrm{M}+\mathrm{Na}]^{+}$244.1313. Found: 244.1315 .

\section{4-Fluorobenzyl 5-(pyridin-2-yl)pentanoate (30)}<smiles>O=C(CCCCc1ccccn1)OCc1ccc(F)cc1</smiles>

2-Vinylpyridine (21 mg, $0.2 \mathrm{mmol})$, 4-fluorobenzyl 3-bromopropanoate (156.7 mg, $0.6 \mathrm{mmol}$ ), $\mathrm{Fe}\left(\mathrm{NH}_{4}\right)_{2}\left(\mathrm{SO}_{4}\right)_{2} \cdot 6 \mathrm{H}_{2} \mathrm{O}$ (1.6 mg, $\left.0.004 \mathrm{mmol}\right), 3,4,7,8$-tetramethyl-1,10-phenanthroline (1 mg, 0.004 $\mathrm{mmol}$ ), $\mathrm{Zn}$ dust (78 mg, $1.2 \mathrm{mmol}$ ) and NaOAc ( $8.2 \mathrm{mg}, 0.1 \mathrm{mmol})$, in $0.4 \mathrm{~mL} 2 \mathrm{wt} \% \mathrm{TPGS}-750-\mathrm{M} / \mathrm{H}_{2} \mathrm{O}$ with $\mathrm{MeCN}(0.04 \mathrm{~mL})$ as co-solvent were reacted at $\mathrm{rt}$ for $16 \mathrm{~h}$ yielding $36.2 \mathrm{mg}(63 \%)$ of 4-fluorobenzyl 5-(pyridin-2-yl)pentanoate as a faint yellow oil (hexane/EtOAc: 80/20).

${ }^{1} \mathrm{H}$ NMR (500 MHz, $\left.\mathrm{CDCl}_{3}\right) \delta 8.51(\mathrm{~s}, 1 \mathrm{H}), 7.56(\mathrm{td}, J=7.7,1.7 \mathrm{~Hz}, 1 \mathrm{H}), 7.31(\mathrm{dd}, J=8.5,5.4$ $\mathrm{Hz}, 2 \mathrm{H}), 7.12-7.06(\mathrm{~m}, 2 \mathrm{H}), 7.02(\mathrm{t}, J=8.7 \mathrm{~Hz}, 2 \mathrm{H}), 5.05(\mathrm{~s}, 2 \mathrm{H}), 2.78$ (t, $J=7.4 \mathrm{~Hz}, 2 \mathrm{H}), 2.38$ (t, $J=$ $7.3 \mathrm{~Hz}, 2 \mathrm{H}$ ), 1.74 (ddt, $J=24.0,15.5,7.2 \mathrm{~Hz}, 4 \mathrm{H}$ ).

${ }^{13} \mathrm{C} \mathrm{NMR}\left(126 \mathrm{MHz}, \mathrm{CDCl}_{3}\right) \delta 173.43,163.69,161.79\left(\mathrm{~d},{ }^{1} J_{(\mathrm{C}-\mathrm{F})}=8.8 \mathrm{~Hz}\right), 149.37,136.41,132.06$ $\left(\mathrm{d},{ }^{1} J_{(\mathrm{C}-\mathrm{F})}=8.8 \mathrm{~Hz}\right), 130.33\left(\mathrm{~d},{ }^{1} J_{(\mathrm{C}-\mathrm{F})}=8.8 \mathrm{~Hz}\right), 122.83,121.14,115.64\left(\mathrm{~d},{ }^{1} J_{(\mathrm{C}-\mathrm{F})}=21.4 \mathrm{~Hz}\right), 65.50,38.03$, $34.22,29.27,24.72$.

${ }^{19} \mathrm{~F}$ NMR $\left(376 \mathrm{MHz}, \mathrm{CDCl}_{3}\right) \delta-113,81$.

HRMS(ESI): Calcd. for $\mathrm{C}_{17} \mathrm{H}_{19} \mathrm{FNO}_{2}[\mathrm{M}+\mathrm{H}]^{+}$288.1400. Found: 288.1407 .

\section{Ethyl 6-(pyridin-2-yl)hexanoate (31)}

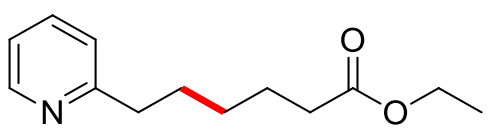

2-Vinylpyridine (21 mg, $0.2 \mathrm{mmol}$ ), ethyl 4-bromobutanoate (117 mg, $0.6 \mathrm{mmol}$ ), $\mathrm{Fe}\left(\mathrm{NH}_{4}\right)_{2}\left(\mathrm{SO}_{4}\right)_{2} \cdot 6 \mathrm{H}_{2} \mathrm{O}(1.6 \mathrm{mg}, 0.004 \mathrm{mmol}), 3,4,7,8$-tetramethyl-1,10-phenanthroline (1 mg, 0.004 mmol), Zn dust (78 mg, $1.2 \mathrm{mmol}$ ) and NaOAc (8.2 mg, $0.1 \mathrm{mmol})$, in $0.4 \mathrm{~mL} 2 \mathrm{wt} \% \mathrm{TPGS}-750-\mathrm{M} / \mathrm{H}_{2} \mathrm{O}$ 
with $\mathrm{MeCN}(0.04 \mathrm{~mL})$ as co-solvent were reacted at $\mathrm{rt}$ for $16 \mathrm{~h}$ yielding $33.2 \mathrm{mg}$ (75\%) of ethyl 6-(pyridin2-yl)hexanoate as a colorless oil (hexane/EtOAc: 80/20).

${ }^{1} \mathrm{H}$ NMR $\left(500 \mathrm{MHz}, \mathrm{CDCl}_{3}\right) \delta 8.49(\mathrm{~d}, J=4.8 \mathrm{~Hz}, 1 \mathrm{H}), 7.55(\mathrm{td}, J=7.7,1.7 \mathrm{~Hz}, 1 \mathrm{H}), 7.10(\mathrm{~d}, J=$ $7.8 \mathrm{~Hz}, 1 \mathrm{H}), 7.08-7.03(\mathrm{~m}, 1 \mathrm{H}), 4.08(\mathrm{q}, J=7.1 \mathrm{~Hz}, 2 \mathrm{H}), 2.78-2.72(\mathrm{~m}, 2 \mathrm{H}), 2.27(\mathrm{t}, J=7.5 \mathrm{~Hz}, 2 \mathrm{H})$, $1.72(\mathrm{p}, J=7.7 \mathrm{~Hz}, 2 \mathrm{H}), 1.64(\mathrm{p}, J=7.6 \mathrm{~Hz}, 2 \mathrm{H}), 1.40-1.33(\mathrm{~m}, 2 \mathrm{H}), 1.21(\mathrm{t}, J=7.1 \mathrm{~Hz}, 3 \mathrm{H})$.

${ }^{13} \mathrm{C}$ NMR $\left(126 \mathrm{MHz}, \mathrm{CDCl}_{3}\right) \delta 173.80,162.22,149.30,136.32,122.77,121.00,60.25,38.26$, $34.35,29.57,28.92,24.91,14.33$.

HRMS(ESI): Calcd. for $\mathrm{C}_{13} \mathrm{H}_{19} \mathrm{NO}_{2}[\mathrm{M}+\mathrm{H}]^{+}$222.1494. Found: 222.1499.

\section{2-(5-((t-Butyldimethylsilyl)oxy)pentyl)pyridine (32)}

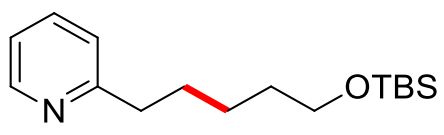

2-Vinylpyridine (21 mg, $0.2 \mathrm{mmol})$, (3-bromopropoxy)(t-butyl)dimethylsilane (152 mg, 0.6 mmol), $\mathrm{Fe}\left(\mathrm{NH}_{4}\right)_{2}\left(\mathrm{SO}_{4}\right)_{2} \cdot 6 \mathrm{H}_{2} \mathrm{O}(1.6 \mathrm{mg}, 0.004 \mathrm{mmol})$, 3,4,7,8-tetramethyl-1,10-phenanthroline (1 mg, $0.004 \mathrm{mmol}), \mathrm{Zn}$ dust (78 mg, $1.2 \mathrm{mmol})$ and NaOAc $(8.2 \mathrm{mg}, 0.1 \mathrm{mmol})$, in $0.4 \mathrm{~mL} 2 \mathrm{wt} \%$ TPGS-750$\mathrm{M} / \mathrm{H}_{2} \mathrm{O}$ with $\mathrm{MeCN}(0.04 \mathrm{~mL})$ as co-solvent were reacted at $\mathrm{rt}$ for $16 \mathrm{~h}$ yielding $34.1 \mathrm{mg}(61 \%)$ of 2-(5((t-butyldimethylsilyl)oxy)pentyl)pyridine as a colorless oil (hexane/EtOAc: 80/20).

${ }^{1} \mathrm{H}$ NMR $\left(500 \mathrm{MHz}, \mathrm{CDCl}_{3}\right) \delta 8.49(\mathrm{~d}, J=4.7 \mathrm{~Hz}, 1 \mathrm{H}), 7.53(\mathrm{t}, J=7.6 \mathrm{~Hz}, 1 \mathrm{H}), 7.10(\mathrm{~d}, J=7.8$ $\mathrm{Hz}, 1 \mathrm{H}), 7.08-7.02(\mathrm{~m}, 1 \mathrm{H}), 3.57(\mathrm{t}, J=6.5 \mathrm{~Hz}, 2 \mathrm{H}), 2.78-2.73(\mathrm{~m}, 2 \mathrm{H}), 1.72(\mathrm{p}, J=7.7 \mathrm{~Hz}, 2 \mathrm{H}), 1.53$ $(\mathrm{p}, J=6.7 \mathrm{~Hz}, 2 \mathrm{H}), 1.40-1.33(\mathrm{~m}, 2 \mathrm{H}), 0.85(\mathrm{~s}, 9 \mathrm{H}), 0.00(\mathrm{~s}, 6 \mathrm{H})$.

${ }^{13} \mathrm{C} \mathrm{NMR}\left(126 \mathrm{MHz}, \mathrm{CDCl}_{3}\right) \delta 162.43,149.30,136.25,122.76,120.92,63.20,38.52,32.82,29.78$, $26.06,25.71,18.43,-5.18$.

HRMS(ESI): Calcd. for $\mathrm{C}_{16} \mathrm{H}_{30} \mathrm{ONSi}[\mathrm{M}+\mathrm{H}]^{+}$280.2097. Found: 280.2084 .

\section{2-(Hept-6-yn-1-yl)pyridine (33)}

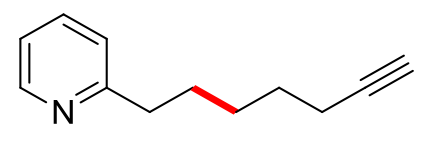


2-Vinylpyridine $\quad(21 \mathrm{mg}, \quad 0.2 \mathrm{mmol}), \quad 5$-iodopent-1-yne $(116.4 \mathrm{mg}, \quad 0.6 \mathrm{mmol})$, $\mathrm{Fe}\left(\mathrm{NH}_{4}\right)_{2}\left(\mathrm{SO}_{4}\right)_{2} \cdot 6 \mathrm{H}_{2} \mathrm{O}(1.6 \mathrm{mg}, 0.004 \mathrm{mmol}), 3,4,7,8$-tetramethyl-1,10-phenanthroline (1 mg, 0.004 mmol), Zn dust (78 mg, $1.2 \mathrm{mmol}$ ) and NaOAc (8.2 mg, $0.1 \mathrm{mmol})$, in $0.4 \mathrm{~mL} 2 \mathrm{wt} \%$ TPGS-750-M/ $\mathrm{H}_{2} \mathrm{O}$ with $\mathrm{MeCN}(0.04 \mathrm{~mL})$ as co-solvent were reacted at $\mathrm{rt}$ for $16 \mathrm{~h}$ yielding $25.3 \mathrm{mg}$ (73\%) of 2-(hept-6-yn1-yl)pyridine as a faint yellow oil (hexane/EtOAc: 80/20).

${ }^{1} \mathrm{H}$ NMR $\left(500 \mathrm{MHz}, \mathrm{CDCl}_{3}\right) \delta 8.50(\mathrm{~d}, J=4.2 \mathrm{~Hz}, 1 \mathrm{H}), 7.56(\mathrm{t}, J=7.6 \mathrm{~Hz}, 1 \mathrm{H}), 7.11(\mathrm{~d}, J=7.7$ $\mathrm{Hz}, 1 \mathrm{H}), 7.10-7.04(\mathrm{~m}, 1 \mathrm{H}), 2.81-2.74(\mathrm{~m}, 2 \mathrm{H}), 2.17(\mathrm{td}, J=6.7,2.2 \mathrm{~Hz}, 2 \mathrm{H}), 1.91(\mathrm{~s}, 1 \mathrm{H}), 1.73(\mathrm{p}, J$ $=7.5 \mathrm{~Hz}, 2 \mathrm{H}), 1.56(\mathrm{p}, J=7.1 \mathrm{~Hz}, 2 \mathrm{H}), 1.46(\mathrm{p}, J=7.5,6.9 \mathrm{~Hz}, 2 \mathrm{H})$.

${ }^{13} \mathrm{C} \mathrm{NMR}\left(126 \mathrm{MHz}, \mathrm{CDCl}_{3}\right) \delta 162.27,149.34,136.32,122.78,121.01,84.65,68.30,38.36,29.43$, $28.58,28.43,18.43$.

HRMS(EI): Calcd. for $\mathrm{C}_{12} \mathrm{H}_{14} \mathrm{~N}[\mathrm{M}-\mathrm{H}]^{+}$172.1126. Found: 172.1126.

\section{(R)-Benzyl 2-((t-butoxycarbonyl)amino)-5-(pyridin-2-yl)pentanoate (34)}

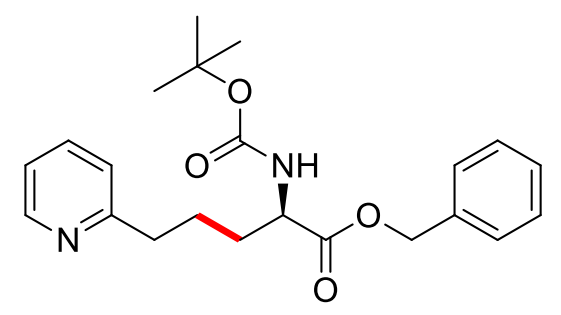

2-Vinylpyridine (21 mg, $0.2 \mathrm{mmol})$, (S)-benzyl 2-((t-butoxycarbonyl)amino)-3-iodopropanoate $(243 \mathrm{mg}, 0.6 \mathrm{mmol}), \mathrm{Fe}\left(\mathrm{NH}_{4}\right)_{2}\left(\mathrm{SO}_{4}\right)_{2} \cdot 6 \mathrm{H}_{2} \mathrm{O}$ (1.6 mg, $\left.0.004 \mathrm{mmol}\right)$, 3,4,7,8-tetramethyl-1,10phenanthroline ( $1 \mathrm{mg}, 0.004 \mathrm{mmol}), \mathrm{Zn}$ dust $(78 \mathrm{mg}, 1.2 \mathrm{mmol})$ and $\mathrm{NaOAc}(8.2 \mathrm{mg}, 0.1 \mathrm{mmol})$, in 0.8 $\mathrm{mL} 2$ wt \% TPGS-750-M/ $\mathrm{H}_{2} \mathrm{O}$ with $\mathrm{MeCN}(0.08 \mathrm{~mL})$ as co-solvent were reacted at $\mathrm{rt}$ for $16 \mathrm{~h}$ yielding $61.5 \mathrm{mg}(80 \%)$ of $(R)$-benzyl 2-((t-butoxycarbonyl)amino)-5-(pyridin-2-yl)pentanoate as a yellow oil (hexane/EtOAc: 50/50).

${ }^{1} \mathrm{H}$ NMR $\left(500 \mathrm{MHz} \mathrm{CDCl}_{3}\right) \delta 8.56-8.41(\mathrm{~m}, 1 \mathrm{H}), 7.55(\mathrm{t}, J=7.5 \mathrm{~Hz}, 1 \mathrm{H}), 7.44-7.27(\mathrm{~m}, 6 \mathrm{H})$, $7.11-7.04(\mathrm{~m}, 2 \mathrm{H}), 5.18-5.08(\mathrm{~m}, 2 \mathrm{H}), 4.42-4.29(\mathrm{~m}, 1 \mathrm{H}), 2.87-2.67(\mathrm{~m}, 2 \mathrm{H}), 1.90-1.66(\mathrm{~m}, 4 \mathrm{H})$, $1.42(\mathrm{~s}, 9 \mathrm{H})$.

${ }^{13} \mathrm{C}$ NMR $\left(126 \mathrm{MHz}, \mathrm{CDCl}_{3}\right) \delta 172.72,161.34,155.51,149.31,136.44,135.55,128.65,128.43$, $128.32,122.90,121.21,79.89,67.04,53.55,37.60,32.16,28.42,25.42$. 
HRMS(ESI): Calcd. for $\mathrm{C}_{22} \mathrm{H}_{29} \mathrm{O}_{4} \mathrm{~N}_{2}[\mathrm{M}+\mathrm{H}]^{+}$385.2127. Found: 385.2139 .

\section{Ethyl 5-(5-(trifluoromethyl)pyridin-2-yl)pentanoate (35)}

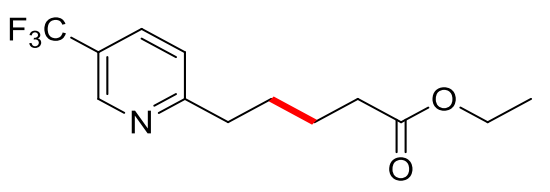

5-(Trifluoromethyl)-2-vinylpyridine (34.6 mg, $0.2 \mathrm{mmol}$ ), ethyl 3-bromopropanoate (108.6 mg, $0.6 \mathrm{mmol}), \mathrm{Fe}\left(\mathrm{NH}_{4}\right)_{2}\left(\mathrm{SO}_{4}\right)_{2} \cdot 6 \mathrm{H}_{2} \mathrm{O}(1.6 \mathrm{mg}, 0.004 \mathrm{mmol}), 3,4,7,8$-tetramethyl-1,10-phenanthroline (1 mg, $0.004 \mathrm{mmol}$ ), Zn dust (78 mg, $1.2 \mathrm{mmol}$ ) and NaOAc ( $8.2 \mathrm{mg}, 0.1 \mathrm{mmol})$, in $0.4 \mathrm{~mL} 2 \mathrm{wt} \%$ TPGS-750$\mathrm{M} / \mathrm{H}_{2} \mathrm{O}$ with $\mathrm{MeCN}(0.04 \mathrm{~mL})$ as co-solvent were reacted at $\mathrm{rt}$ for $16 \mathrm{~h}$ yielding $33.1 \mathrm{mg}(60 \%)$ of ethyl 5-(5-(trifluoromethyl)pyridin-2-yl)pentanoate as a faint yellow oil (hexane/EtOAc: 80/20).

${ }^{1} \mathrm{H}$ NMR (500 MHz, $\left.\mathrm{CDCl}_{3}\right) \delta 8.77(\mathrm{~s}, 1 \mathrm{H}), 7.82(\mathrm{~m}, 1 \mathrm{H}), 7.27(\mathrm{~d}, J=10 \mathrm{~Hz}, 1 \mathrm{H}), 4.11(\mathrm{q}, J=$ $5 \mathrm{~Hz}, 2 \mathrm{H}), 2.87$ (t, $J=8 \mathrm{~Hz}, 2 \mathrm{H}), 2.33$ (t, $J=8 \mathrm{~Hz}, 2 \mathrm{H}), 1.79(\mathrm{~m}, 2 \mathrm{H}), 1.68(\mathrm{~m}, 2 \mathrm{H}), 1.23(\mathrm{t}, J=7 \mathrm{~Hz}$, $3 \mathrm{H})$;

${ }^{13} \mathrm{C}$ NMR $\left(126 \mathrm{MHz}, \mathrm{CDCl}_{3}\right) \delta 173.55,165.98,146.3\left(\mathrm{q},{ }^{1} J_{(\mathrm{C}-\mathrm{F})}=2.5 \mathrm{~Hz}\right), 133.51\left(\mathrm{q},{ }^{1} J_{(\mathrm{C}-\mathrm{F})}=3.8\right.$ $\mathrm{Hz}), 124.94\left(\mathrm{q},{ }^{1} J_{(\mathrm{C}-\mathrm{F})}=270 \mathrm{~Hz}\right), 124.5\left(\mathrm{q},{ }^{1} J_{(\mathrm{C}-\mathrm{F})}=33.8 \mathrm{~Hz}\right), 122.58,60.41,38.05,34.18,28.99,24.65$, 14.35 ;

${ }^{19} \mathrm{~F} \mathrm{NMR}\left(376 \mathrm{MHz}, \mathrm{CDCl}_{3}\right) \delta-62.32$.

HRMS(ESI): Calcd. for $\mathrm{C}_{13} \mathrm{H}_{16} \mathrm{~F}_{3} \mathrm{NO}_{2} \mathrm{Na}[\mathrm{M}+\mathrm{Na}]^{+}$298.1031. Found: 298.1030 .

\section{2-(5-Phenoxypentyl)-5-(trifluoromethyl)pyridine (36)}

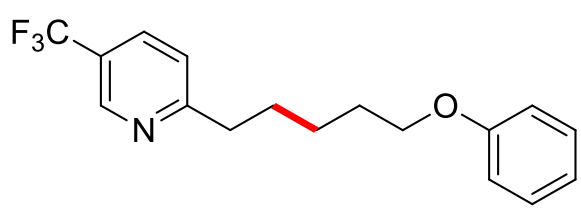

5-(Trifluoromethyl)-2-vinylpyridine (34.6 mg, $0.2 \mathrm{mmol}$ ), (3-bromopropoxy)benzene (129.1 mg, $0.6 \mathrm{mmol}), \mathrm{Fe}\left(\mathrm{NH}_{4}\right)_{2}\left(\mathrm{SO}_{4}\right)_{2} \cdot 6 \mathrm{H}_{2} \mathrm{O}(1.6 \mathrm{mg}, 0.004 \mathrm{mmol})$, 3,4,7,8-tetramethyl-1,10-phenanthroline (1 mg, $0.004 \mathrm{mmol}$ ), Zn dust (78 mg, $1.2 \mathrm{mmol}$ ) and NaOAc ( $8.2 \mathrm{mg}, 0.1 \mathrm{mmol})$, in $0.4 \mathrm{~mL} 2 \mathrm{wt} \%$ TPGS-750$\mathrm{M} / \mathrm{H}_{2} \mathrm{O}$ with $\mathrm{MeCN}(0.04 \mathrm{~mL})$ as co-solvent were reacted at $\mathrm{rt}$ for $16 \mathrm{~h}$ yielding $40.0 \mathrm{mg}(63 \%)$ of 2-(5- 
phenoxypentyl)-5-(trifluoromethyl)pyridine as a faint yellow oil (hexane/EtOAc: 80/20).

${ }^{1} \mathrm{H}$ NMR (500 MHz, $\left.\mathrm{CDCl}_{3}\right) \delta 8.80(\mathrm{~s}, 1 \mathrm{H}), 7.83(\mathrm{~d}, J=8 \mathrm{~Hz}, 1 \mathrm{H}), 7.28(\mathrm{~m}, 3 \mathrm{H}), 6.93(\mathrm{t}, J=7.5$ $\mathrm{Hz}, 1 \mathrm{H}), 6.89$ (d, $J=8.5 \mathrm{~Hz}, 2 \mathrm{H}), 3.96(\mathrm{t}, J=6 \mathrm{~Hz}, 2 \mathrm{H}), 2.90$ (t, $J=7.5 \mathrm{~Hz}, 2 \mathrm{H}), 1.84$ (m, $4 \mathrm{H}), 1.56$ (m, $2 \mathrm{H})$;

${ }^{13} \mathrm{C}$ NMR $\left(126 \mathrm{MHz}, \mathrm{CDCl}_{3}\right) \delta 166.3,159.1,146.3\left(\mathrm{q},{ }^{1} J_{(\mathrm{C}-\mathrm{F})}=4 \mathrm{~Hz}\right), 133.5\left(\mathrm{q},{ }^{1} J_{(\mathrm{C}-\mathrm{F})}=3.4 \mathrm{~Hz}\right)$, $129.5,125.0\left(\mathrm{q},{ }^{1} J_{(\mathrm{C}-\mathrm{F})}=270 \mathrm{~Hz}\right), 124.1\left(\mathrm{q},{ }^{1} J_{(\mathrm{C}-\mathrm{F})}=32.6 \mathrm{~Hz}\right), 122.6,120.7,114.6,67.7,38.4,29.4,29.2$, 25.9 ;

${ }^{19} \mathrm{~F}$ NMR $\left(376 \mathrm{MHz}, \mathrm{CDCl}_{3}\right) \delta-62.25$.

HRMS(EI): Calcd. for $\mathrm{C}_{17} \mathrm{H}_{18} \mathrm{~F}_{3} \mathrm{NO}[\mathrm{M}]^{+}$309.1341. Found: 309.1342 .

\section{N-(4-Chloro-3-(trifluoromethyl)phenyl)-5-(5-(trifluoromethyl)pyridin-2-yl)pentanamide (37)}

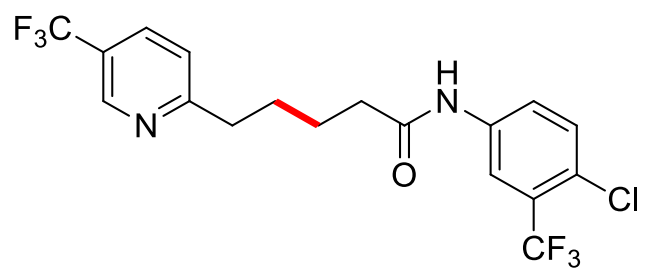

5-(Trifluoromethyl)-2-vinylpyridine $\quad(34.6 \quad \mathrm{mg}, \quad 0.2 \quad \mathrm{mmol}), \quad 3$-bromo- $N$-(4-chloro-3(trifluoromethyl)phenyl)propanamide (198.3 mg, $0.6 \mathrm{mmol}), \mathrm{Fe}\left(\mathrm{NH}_{4}\right)_{2}\left(\mathrm{SO}_{4}\right)_{2} \cdot 6 \mathrm{H}_{2} \mathrm{O}(1.6 \mathrm{mg}, 0.004$ mmol), 3,4,7,8-tetramethyl-1,10-phenanthroline (1 mg, $0.004 \mathrm{mmol}), \mathrm{Zn}$ dust (78 mg, $1.2 \mathrm{mmol})$ and NaOAc (8.2 mg, $0.1 \mathrm{mmol})$, in $0.4 \mathrm{~mL} 2 \mathrm{wt} \%$ TPGS-750-M/ $\mathrm{H}_{2} \mathrm{O}$ with $\mathrm{MeCN}(0.04 \mathrm{~mL})$ as co-solvent were reacted at $\mathrm{rt}$ for $16 \mathrm{~h}$ yielding $56.9 \mathrm{mg}$ (67\%) of $\mathrm{N}$-(4-chloro-3-(trifluoromethyl)phenyl)-5-(5(trifluoromethyl)pyridin-2-yl)pentanamide as a faint yellow oil (hexane/EtOAc: 70/30).

${ }^{1} \mathrm{H}$ NMR $\left(500 \mathrm{MHz}, \mathrm{CDCl}_{3}\right) \delta 8.75(\mathrm{~s}, 1 \mathrm{H}), 8.06(\mathrm{br}, 1 \mathrm{H}), 7.83(\mathrm{~m}, 2 \mathrm{H}), 7.73(\mathrm{~d}, J=8.5 \mathrm{~Hz}$, $1 \mathrm{H}), 7.40(\mathrm{~d}, J=8.5 \mathrm{~Hz}, 1 \mathrm{H}), 7.28(\mathrm{~d}, J=8.5 \mathrm{~Hz}, 1 \mathrm{H}), 2.90(\mathrm{t}, J=8 \mathrm{~Hz}, 2 \mathrm{H}), 2.42(\mathrm{t}, J=7 \mathrm{~Hz}, 2 \mathrm{H})$, $1.79(\mathrm{~m}, 4 \mathrm{H})$;

${ }^{13} \mathrm{C}$ NMR $\left(126 \mathrm{MHz}, \mathrm{CDCl}_{3}\right) \delta 171.6,165.7,146.2\left(\mathrm{q},{ }^{1} J_{(\mathrm{C}-\mathrm{F})}=4.2 \mathrm{~Hz}\right), 137.0,133.8\left(\mathrm{q},{ }^{1} J_{(\mathrm{C}-\mathrm{F})}=\right.$ $3.4 \mathrm{~Hz}), 132.1,128.9\left(\mathrm{q},{ }^{1} J_{(\mathrm{C}-\mathrm{F})}=32 \mathrm{~Hz}\right), 126.9\left(\mathrm{q},{ }^{1} J_{(\mathrm{C}-\mathrm{F})}=252 \mathrm{~Hz}\right), 124.7\left(\mathrm{q},{ }^{1} J_{(\mathrm{C}-\mathrm{F})}=32.9 \mathrm{~Hz}\right), 124.2(\mathrm{q}$, $\left.{ }^{1} J_{(\mathrm{C}-\mathrm{F})}=186 \mathrm{~Hz}\right), 123.9,123.7\left(\mathrm{q},{ }^{1} J_{(\mathrm{C}-\mathrm{F})}=272 \mathrm{~Hz}\right), 122.9,118.9\left(\mathrm{q},{ }^{1} J_{(\mathrm{C}-\mathrm{F})}=5.4 \mathrm{~Hz}\right), 37.7,37.2,28.7$, 24.8 
${ }^{19} \mathrm{~F}$ NMR $\left(376 \mathrm{MHz}, \mathrm{CDCl}_{3}\right) \delta-62.10,-62.66$.

HRMS(ESI): Calcd. for $\mathrm{C}_{18} \mathrm{H}_{15} \mathrm{ClF}_{6} \mathrm{~N}_{2} \mathrm{ONa}[\mathrm{M}+\mathrm{Na}]^{+}$447.0675. Found: 447.0681.

\section{2-Methyl-3-(5-phenoxypentyl)pyridine (38)}

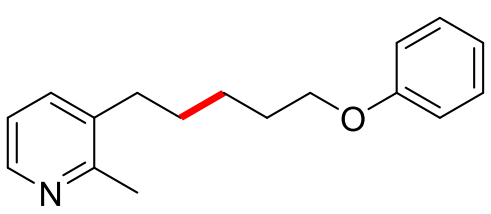

2-Methyl-3-vinylpyridine (23.8 mg, $0.2 \mathrm{mmol}$ ), (3-bromopropoxy)benzene (129.1 mg, $0.6 \mathrm{mmol}$ ), $\mathrm{Fe}\left(\mathrm{NH}_{4}\right)_{2}\left(\mathrm{SO}_{4}\right)_{2} \cdot 6 \mathrm{H}_{2} \mathrm{O}(1.6 \mathrm{mg}, 0.004 \mathrm{mmol}), 3,4,7,8$-tetramethyl-1,10-phenanthroline (1 mg, 0.004 mmol), Zn dust (78 mg, $1.2 \mathrm{mmol}$ ) and NaOAc (8.2 mg, $0.1 \mathrm{mmol})$, in $0.4 \mathrm{~mL} 2$ wt \% TPGS-750-M/ $\mathrm{H}_{2} \mathrm{O}$ with $\mathrm{MeCN}(0.04 \mathrm{~mL})$ as co-solvent were reacted at $\mathrm{rt}$ for $16 \mathrm{~h}$ yielding $33.8 \mathrm{mg}(66 \%)$ of 2-methyl-3-(5phenoxypentyl)pyridine as a light yellow oil (hexane/EtOAc: 80/20).

${ }^{1} \mathrm{H}$ NMR $\left(500 \mathrm{MHz}, \mathrm{CDCl}_{3}\right) \delta$ 8.40-8.31 (m, 1H), $7.45(\mathrm{~d}, J=7.4 \mathrm{~Hz}, 1 \mathrm{H}), 7.33-7.22(\mathrm{~m}, 2 \mathrm{H})$, 7.15-7.05 (m, 1H), $6.94(\mathrm{t}, J=7.4 \mathrm{~Hz}, 1 \mathrm{H}), 6.89(\mathrm{~d}, J=7.9 \mathrm{~Hz}, 2 \mathrm{H}), 3.97(\mathrm{t}, J=6.4 \mathrm{~Hz}, 2 \mathrm{H}), 2.64(\mathrm{t}, J=$ $7.8 \mathrm{~Hz}, 2 \mathrm{H}), 2.57(\mathrm{~s}, 3 \mathrm{H}), 1.89-1.80(\mathrm{~m}, 2 \mathrm{H}), 1.71-1.62(\mathrm{~m}, 2 \mathrm{H}), 1.62-1.52(\mathrm{~m}, 2 \mathrm{H})$.

${ }^{13} \mathrm{C} \mathrm{NMR}\left(126 \mathrm{MHz}, \mathrm{CDCl}_{3}\right) \delta 159.1,156.4,146.1,137.0,136.1,129.6,121.6,120.7,114.6,67.7$, $32.7,29.6,29.3,26.2,22.0$.

HRMS(EI): Calcd. for $\mathrm{C}_{17} \mathrm{H}_{21} \mathrm{NO}[\mathrm{M}]^{+}$255.1623. Found: 255.1626 .

\section{Ethyl 5-(2-methylpyridin-3-yl)pentanoate (39)}

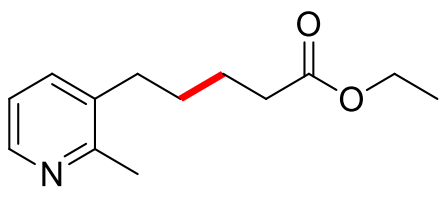

2-Methyl-3-vinylpyridine (23.8 mg, $0.2 \mathrm{mmol})$, ethyl 3-bromopropanoate (108.6 mg, $0.6 \mathrm{mmol})$, $\mathrm{Fe}\left(\mathrm{NH}_{4}\right)_{2}\left(\mathrm{SO}_{4}\right)_{2} \cdot 6 \mathrm{H}_{2} \mathrm{O}(1.6 \mathrm{mg}, 0.004 \mathrm{mmol}), 3,4,7,8$-tetramethyl-1,10-phenanthroline (1 mg, 0.004 mmol), Zn dust (78 mg, $1.2 \mathrm{mmol})$ and NaOAc (8.2 mg, $0.1 \mathrm{mmol})$, in $0.4 \mathrm{~mL} 2 \mathrm{wt} \% \mathrm{TPGS}-750-\mathrm{M} / \mathrm{H}_{2} \mathrm{O}$ with $\mathrm{MeCN}(0.04 \mathrm{~mL})$ as co-solvent were reacted at $\mathrm{rt}$ for $16 \mathrm{~h}$ yielding $29.9 \mathrm{mg}(68 \%)$ of ethyl 5-(2methylpyridin-3-yl)pentanoate as a faint yellow liquid (hexane/ EtOAc: 80/20). 
${ }^{1} \mathrm{H}$ NMR $\left(500 \mathrm{MHz}, \mathrm{CDCl}_{3}\right) \delta 8.32(\mathrm{~d}, J=4.7 \mathrm{~Hz}, 1 \mathrm{H}), 7.38(\mathrm{~d}, J=8 \mathrm{~Hz}, 1 \mathrm{H}), 7.04(\mathrm{~m}, 1 \mathrm{H}), 4.12$ (q, $J=7.1 \mathrm{~Hz}, 2 \mathrm{H}), 2.61(\mathrm{t}, J=7.8 \mathrm{~Hz}, 2 \mathrm{H}), 2.52(\mathrm{~s}, 3 \mathrm{H}), 2.34(\mathrm{t}, J=7.3 \mathrm{~Hz}, 2 \mathrm{H}), 1.70(\mathrm{p}, J=7.3 \mathrm{~Hz}$ 2H), 1.61 (p, $J=7.5,7.0 \mathrm{~Hz}, 2 \mathrm{H}), 1.24(\mathrm{t}, J=7.1 \mathrm{~Hz}, 3 \mathrm{H})$.

${ }^{13} \mathrm{C}$ NMR $\left(126 \mathrm{MHz} \mathrm{CDCl}_{3}\right) \delta 173.42,156.51,146.60,136.20,135.21,121.23,60.30,34.09$, $32.38,29.16,24.76,22.14,14.24$.

HRMS(ESI): Calcd. for $\mathrm{C}_{13} \mathrm{H}_{20} \mathrm{NO}_{2}[\mathrm{M}+\mathrm{H}]^{+}$222.1494. Found: 222.1487.

\section{(9H-Fluoren-9-yl)methyl 5-(2-fluoropyridin-4-yl)pentanoate (40)}

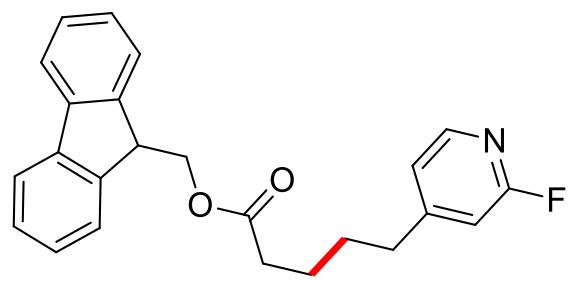

2-Fluoro-4-vinylpyridine (24.6 mg, $0.2 \mathrm{mmol}$ ), (9H-fluoren-9-yl)methyl 3-bromopropanoate (198.7 mg, $0.6 \mathrm{mmol}), \mathrm{Fe}\left(\mathrm{NH}_{4}\right)_{2}\left(\mathrm{SO}_{4}\right)_{2} \cdot 6 \mathrm{H}_{2} \mathrm{O}$ (1.6 mg, $\left.0.004 \mathrm{mmol}\right)$, 3,4,7,8-tetramethyl-1,10phenanthroline (1 mg, $0.004 \mathrm{mmol}), \mathrm{Zn}$ dust (78 $\mathrm{mg}, 1.2 \mathrm{mmol})$ and $\mathrm{NaOAc}(8.2 \mathrm{mg}, 0.1 \mathrm{mmol})$, in 0.4 $\mathrm{mL} 2 \mathrm{wt} \%$ TPGS-750-M/ $\mathrm{H}_{2} \mathrm{O}$ with $\mathrm{MeCN}(0.04 \mathrm{~mL})$ as co-solvent were reacted at $\mathrm{rt}$ for $16 \mathrm{~h}$ yielding $57.7 \mathrm{mg}(76 \%)$ of (9H-fluoren-9-yl)methyl 5-(2-fluoropyridin-4-yl)pentanoate as a colorless oil (hexane/EtOAc: 80/20).

${ }^{1} \mathrm{H}$ NMR $\left(500 \mathrm{MHz}, \mathrm{CDCl}_{3}\right) \delta 8.10(\mathrm{~d}, J=5.1 \mathrm{~Hz}, 1 \mathrm{H}), 7.76(\mathrm{~d}, J=7.6 \mathrm{~Hz}, 2 \mathrm{H}), 7.58(\mathrm{~d}, J=7.5$ $\mathrm{Hz}, 2 \mathrm{H}), 7.40$ (t, $J=7.4 \mathrm{~Hz}, 2 \mathrm{H}), 7.31(\mathrm{td}, J=7.5,1.1 \mathrm{~Hz}, 2 \mathrm{H}), 6.97$ (d, J=5.1 Hz, 1H), $6.72(\mathrm{~s}, 1 \mathrm{H})$, $4.44(\mathrm{~d}, J=6.8 \mathrm{~Hz}, 2 \mathrm{H}), 4.20(\mathrm{t}, J=6.8 \mathrm{~Hz}, 1 \mathrm{H}), 2.62(\mathrm{t}, J=7.0 \mathrm{~Hz}, 2 \mathrm{H}), 2.39(\mathrm{t}, J=6.9 \mathrm{~Hz}, 2 \mathrm{H}), 1.63$ $(\mathrm{m}, 4 \mathrm{H})$.

${ }^{13} \mathrm{C}$ NMR $\left(126 \mathrm{MHz}, \mathrm{CDCl}_{3}\right) \delta 173.11,165.07\left(\mathrm{~d},{ }^{1} J_{(\mathrm{C}-\mathrm{F})}=239.4 \mathrm{~Hz}\right), 157.01\left(\mathrm{~d},{ }^{1} J_{(\mathrm{C}-\mathrm{F})}=7.6 \mathrm{~Hz}\right)$, $147.41\left(\mathrm{~d},{ }^{1} J_{(\mathrm{C}-\mathrm{F})}=16.4 \mathrm{~Hz}\right), 143.75,141.34,127.80,127.08,124.89,121.60\left(\mathrm{~d},{ }^{1} J_{(\mathrm{C}-\mathrm{F})}=3.8 \mathrm{~Hz}\right), 120.04$, $109.20\left(\mathrm{~d},{ }^{1} J_{(\mathrm{C}-\mathrm{F})}=36.5\right), 66.07,46.89,34.73\left(\mathrm{~d},{ }^{1} J_{(\mathrm{C}-\mathrm{F})}=2.5 \mathrm{~Hz}\right), 33.90,29.28,24.32$.

${ }^{19} \mathrm{~F}$ NMR $\left(376 \mathrm{MHz}, \mathrm{CDCl}_{3}\right) \delta-69.07$.

HRMS(ESI): Calcd. for $\mathrm{C}_{24} \mathrm{H}_{22} \mathrm{FNO}_{2} \mathrm{Na}[\mathrm{M}+\mathrm{Na}]^{+}$398.1532. Found: 398.1544 . 


\section{(9H-Fluoren-9-yl)methyl 5-(5-(4-(trifluoromethoxy)phenyl)pyridin-2-yl)pentanoate (41)}

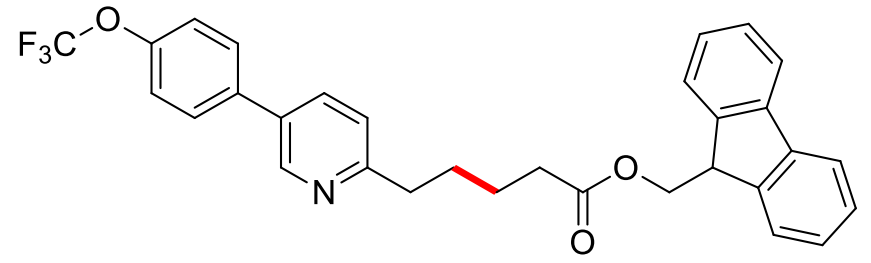

5-(4-(Trifluoromethoxy)phenyl)-2-vinylpyridine (53.1 mg, $0.2 \mathrm{mmol})$, (9H-fluoren-9-yl)methyl 3-bromopropanoate $(198.7 \mathrm{mg}, 0.6 \mathrm{mmol}), \mathrm{Fe}\left(\mathrm{NH}_{4}\right)_{2}\left(\mathrm{SO}_{4}\right)_{2} \cdot 6 \mathrm{H}_{2} \mathrm{O}$ (1.6 mg, $\left.0.004 \mathrm{mmol}\right), 3,4,7,8$ tetramethyl-1,10-phenanthroline (1 mg, $0.004 \mathrm{mmol}), \mathrm{Zn}$ dust $(78 \mathrm{mg}, 1.2 \mathrm{mmol})$ and $\mathrm{NaOAc}(8.2 \mathrm{mg}$, $0.1 \mathrm{mmol})$, in $0.4 \mathrm{~mL} 2 \mathrm{wt} \%$ TPGS-750-M/ $\mathrm{H}_{2} \mathrm{O}$ with $\mathrm{MeCN}(0.04 \mathrm{~mL})$ as co-solvent were reacted at $\mathrm{rt}$ for $16 \mathrm{~h}$ yielding $62.3 \mathrm{mg}(60 \%)$ of (9H-fluoren-9-yl)methyl 5-(5-(4-(trifluoromethoxy)phenyl)pyridin-2yl)pentanoate as a colorless oil (hexane/EtOAc: 80/20).

${ }^{1} \mathrm{H} \mathrm{NMR}\left(500 \mathrm{MHz}, \mathrm{CDCl}_{3}\right) \delta 8.74(\mathrm{~s}, 1 \mathrm{H}), 7.75(\mathrm{~m}, 3 \mathrm{H}), 7.58(\mathrm{~m}, 4 \mathrm{H}), 7.40(\mathrm{t}, J=7.4 \mathrm{~Hz}, 2$ H), $7.31(\mathrm{~m}, 4 \mathrm{H}), 7.23(\mathrm{~d}, J=8 \mathrm{~Hz}, 1 \mathrm{H}), 4.40(\mathrm{~d}, J=7 \mathrm{~Hz}, 2 \mathrm{H}), 4.20$ (t, $J=7 \mathrm{~Hz}, 1 \mathrm{H}), 2.87(\mathrm{t}, J=7.5$ $\mathrm{Hz}, 2 \mathrm{H}), 2.46$ (t, $J=7.3 \mathrm{~Hz}, 2 \mathrm{H}), 1.82(\mathrm{~m}, 4 \mathrm{H})$.

${ }^{13} \mathrm{C}$ NMR $\left(126 \mathrm{MHz}, \mathrm{CDC}_{3}\right) \delta 173.51,161.11,149.15\left(\mathrm{q},{ }^{1} J_{(\mathrm{C}-\mathrm{F})}=1.7 \mathrm{~Hz}\right), 147.60,143.92,141.41$, $136.71,134.85,132.88,128.47,127.86,127.17,125.62\left(\mathrm{q},{ }^{1} J_{(\mathrm{C}-\mathrm{F})}=413 \mathrm{~Hz}\right), 125.10,122.85,121.59$, $120.11,119.57,66.28,46.97,37.63,34.26,29.20,24.68$.

19F NMR (376 MHz, CDCl3) $\delta-57.84$.

HRMS(ESI): Calcd. for $\mathrm{C}_{31} \mathrm{H}_{27} \mathrm{~F}_{3} \mathrm{NO}_{3}[\mathrm{M}+\mathrm{H}]^{+}$518.1943. Found: 518.1954.

\section{2-(2-Cyclopentylethyl)pyridine (42)}

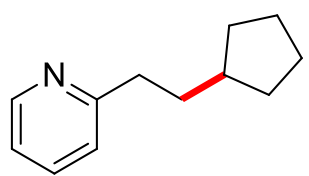

2-Vinylpyridine (21 mg, $0.2 \mathrm{mmol})$, bromocyclopentane $(89.4 \mathrm{mg}, \quad 0.6 \mathrm{mmol})$, $\mathrm{Fe}\left(\mathrm{NH}_{4}\right)_{2}\left(\mathrm{SO}_{4}\right)_{2} \cdot 6 \mathrm{H}_{2} \mathrm{O}(1.6 \mathrm{mg}, 0.004 \mathrm{mmol}), 3,4,7,8$-tetramethyl-1,10-phenanthroline (1 mg, 0.004 mmol), Zn dust (78 mg, $1.2 \mathrm{mmol})$ and NaOAc (8.2 mg, $0.1 \mathrm{mmol})$, in $0.4 \mathrm{~mL} 2 \mathrm{wt} \% \mathrm{TPGS}-750-\mathrm{M} / \mathrm{H}_{2} \mathrm{O}$ with $\mathrm{MeCN}(0.04 \mathrm{~mL})$ as co-solvent were reacted at $\mathrm{rt}$ for $16 \mathrm{~h}$ yielding $27.1 \mathrm{mg}(77 \%)$ of 2-(2cyclopentylethyl)pyridine as a faint yellow oil (hexane/EtOAc: 80/20). 
${ }^{1} \mathrm{H}$ NMR $\left(500 \mathrm{MHz}, \mathrm{CDCl}_{3}\right) \delta 8.53-8.46(\mathrm{~m}, 1 \mathrm{H}), 7.54(\mathrm{t}, J=8.4 \mathrm{~Hz}, 1 \mathrm{H}), 7.11(\mathrm{~d}, J=7.8 \mathrm{~Hz}$, $1 \mathrm{H}), 7.09-7.02(\mathrm{~m}, 1 \mathrm{H}), 2.81-2.74(\mathrm{~m}, 2 \mathrm{H}), 1.81-1.68(\mathrm{~m}, 5 \mathrm{H}), 1.64-1.54(\mathrm{~m}, 2 \mathrm{H}), 1.54-1.43(\mathrm{~m}$, $2 \mathrm{H}), 1.13(\mathrm{~m}, 2 \mathrm{H})$.

${ }^{13} \mathrm{C} \mathrm{NMR}\left(126 \mathrm{MHz}, \mathrm{CDCl}_{3}\right) \delta 162.78,149.27,136.27,122.70,120.87,40.04,37.81,36.47,32.73$, 25.32 .

HRMS(ESI): Calcd. For $\mathrm{C}_{12} \mathrm{H}_{18} \mathrm{~N}[\mathrm{M}+\mathrm{H}]^{+}$176.1439. Found: 176.1435 .

\section{2-(2-Cyclohexylethyl)pyridine (43)}

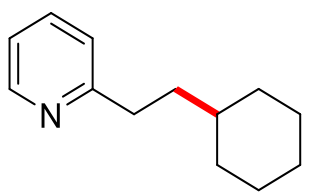

2-Vinylpyridine (21 mg, $0.2 \mathrm{mmol})$, bromocyclohexane $(98 \mathrm{mg}, \quad 0.6 \mathrm{mmol})$, $\mathrm{Fe}\left(\mathrm{NH}_{4}\right)_{2}\left(\mathrm{SO}_{4}\right)_{2} \cdot 6 \mathrm{H}_{2} \mathrm{O}(1.6 \mathrm{mg}, 0.004 \mathrm{mmol}), 3,4,7,8$-tetramethyl-1,10-phenanthroline (1 mg, 0.004 $\mathrm{mmol}$ ), $\mathrm{Zn}$ dust (78 mg, $1.2 \mathrm{mmol}$ ) and $\mathrm{NaOAc}(8.2 \mathrm{mg}, 0.1 \mathrm{mmol})$, in $0.4 \mathrm{~mL} 2 \mathrm{wt} \% \mathrm{TPGS}-750-\mathrm{M} / \mathrm{H}_{2} \mathrm{O}$ with $\mathrm{MeCN}(0.04 \mathrm{~mL})$ as co-solvent were reacted at $\mathrm{rt}$ for $16 \mathrm{~h}$ yielding $27.0 \mathrm{mg}(71 \%)$ of 2-(2cyclohexylethyl)pyridine as a slight yellow oil (hexane/EtOAc: 80/20).

${ }^{1} \mathrm{H}$ NMR $\left(500 \mathrm{MHz}, \mathrm{CDCl}_{3}\right) \delta 8.58-8.47(\mathrm{~m}, 1 \mathrm{H}), 7.57(\mathrm{t}, J=7.6 \mathrm{~Hz}, 1 \mathrm{H}), 7.13(\mathrm{~d}, J=7.7 \mathrm{~Hz}$, 1H), 7.10-7.04 (m, 1H), $2.78(\mathrm{t}, J=8.0 \mathrm{~Hz}, 2 \mathrm{H}), 1.82-1.53(\mathrm{~m}, 7 \mathrm{H}), 1.34-1.07(\mathrm{~m}, 4 \mathrm{H}), 1.01-0.86(\mathrm{~m}, 2 \mathrm{H})$.

${ }^{13} \mathrm{C} \mathrm{NMR}\left(126 \mathrm{MHz}, \mathrm{CDCl}_{3}\right) \delta 163.0,149.2,136.4,122.8,120.9,37.8,37.7,36.0,33.4,26.8$, 26.5.

HRMS(EI): Calcd. for $\mathrm{C}_{13} \mathrm{H}_{19} \mathrm{~N}[\mathrm{M}]^{+}$189.1517. Found: 189.1508 .

\section{2-(3-Methylpentyl)pyridine (44)}

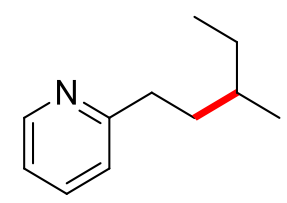

2-Vinylpyridine (21 mg, $0.2 \mathrm{mmol}), 2$-bromobutane (82.2 mg, $0.6 \mathrm{mmol}), \mathrm{Fe}\left(\mathrm{NH}_{4}\right)_{2}\left(\mathrm{SO}_{4}\right)_{2} \cdot 6 \mathrm{H}_{2} \mathrm{O}$ (1.6 mg, $0.004 \mathrm{mmol}$ ), 3,4,7,8-tetramethyl-1,10-phenanthroline (1 mg, $0.004 \mathrm{mmol}), \mathrm{Zn}$ dust (78 mg, 1.2 
mmol) and $\mathrm{NaOAc}(8.2 \mathrm{mg}, 0.1 \mathrm{mmol})$, in $0.4 \mathrm{~mL} 2 \mathrm{wt} \% \mathrm{TPGS}-750-\mathrm{M} / \mathrm{H}_{2} \mathrm{O}$ with $\mathrm{MeCN}(0.04 \mathrm{~mL})$ as co-solvent were reacted at $\mathrm{rt}$ for $16 \mathrm{~h}$ yielding $24.3 \mathrm{mg}$ (74\%) of 2-(3-methylpentyl)pyridine as a faint yellow oil (hexane/EtOAc: 80/20).

${ }^{1} \mathrm{H}$ NMR $\left(500 \mathrm{MHz}, \mathrm{CDCl}_{3}\right) \delta 8.51(\mathrm{~d}, J=4.7 \mathrm{~Hz}, 1 \mathrm{H}), 7.56(\mathrm{td}, J=7.7,1.8 \mathrm{~Hz}, 1 \mathrm{H}), 7.13(\mathrm{~d}, J$ $=7.8 \mathrm{~Hz}, 1 \mathrm{H}), 7.11-7.04(\mathrm{~m}, 1 \mathrm{H}), 2.81(\mathrm{ddd}, J=13.6,10.8,5.6 \mathrm{~Hz}, 1 \mathrm{H}), 2.73(\mathrm{ddd}, J=13.6,10.6,5.9$ $\mathrm{Hz}, 1 \mathrm{H}), 1.74$ (ddt, $J=13.3,10.8,5.5 \mathrm{~Hz}, 1 \mathrm{H}), 1.52$ (dddd, $J=13.2,10.7,7.4,5.7 \mathrm{~Hz}, 1 \mathrm{H}$ ), $1.43-1.38$ (m, 2H), 1.19 (dt, $J=14.9,7.5 \mathrm{~Hz}, 1 \mathrm{H}), 0.93(\mathrm{~d}, J=6.3 \mathrm{~Hz}, 3 \mathrm{H}), 0.87(\mathrm{t}, J=7.2 \mathrm{~Hz}, 3 \mathrm{H})$.

${ }^{13} \mathrm{C} \mathrm{NMR}\left(126 \mathrm{MHz}, \mathrm{CDCl}_{3}\right) \delta 162.98,149.32,136.33,122.72,120.90,36.95,36.28,34.46,29.49$, 19.20, 11.46.

HRMS(EI): Calcd. For $\mathrm{C}_{11} \mathrm{H}_{17} \mathrm{~N}[\mathrm{M}]^{+}$163.1361. Found: 163.1360 .

\section{2-(2-(Tetrahydro-2H-pyran-4-yl)ethyl)pyridine (45)}

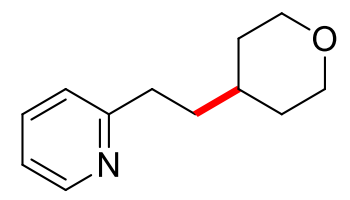

2-Vinylpyridine (21 $\mathrm{mg}, \quad 0.2 \mathrm{mmol}$ ), 4-bromotetrahydropyran (99 $\mathrm{mg}, \quad 0.6 \mathrm{mmol}$ ), $\mathrm{Fe}\left(\mathrm{NH}_{4}\right)_{2}\left(\mathrm{SO}_{4}\right)_{2} \cdot 6 \mathrm{H}_{2} \mathrm{O}$ (1.6 mg, $\left.0.004 \mathrm{mmol}\right), 3,4,7,8$-tetramethyl-1,10-phenanthroline (1 mg, 0.004 $\mathrm{mmol}$ ), $\mathrm{Zn}$ dust (78 mg, $1.2 \mathrm{mmol}$ ) and NaOAc ( $8.2 \mathrm{mg}, 0.1 \mathrm{mmol})$, in $0.4 \mathrm{~mL} 2 \mathrm{wt} \% \mathrm{TPGS}-750-\mathrm{M} / \mathrm{H}_{2} \mathrm{O}$ with $\mathrm{MeCN}(0.04 \mathrm{~mL})$ as co-solvent were reacted at $\mathrm{rt}$ for $16 \mathrm{~h}$ yielding $23.4 \mathrm{mg}(61 \%)$ of 2-(2-(tetrahydro2H-pyran-4-yl)ethyl)pyridine as a colorless oil (hexane/EtOAc: 50/50).

${ }^{1} \mathrm{H}$ NMR $\left(500 \mathrm{MHz}, \mathrm{CDCl}_{3}\right) \delta 8.55-8.44(\mathrm{~m}, 1 \mathrm{H}), 7.55(\mathrm{td}, J=7.7,1.7 \mathrm{~Hz}, 1 \mathrm{H}), 7.21-7.03(\mathrm{~m}$, 2H), 3.92 (dd, $J=10.8,3.2 \mathrm{~Hz}, 2 \mathrm{H}), 3.33(\mathrm{td}, J=11.9,1.8 \mathrm{~Hz}, 2 \mathrm{H}), 2.81-2.74(\mathrm{~m}, 2 \mathrm{H}), 1.69-1.60$ (m, 4H), 1.50 (dtt, $J=14.1,6.9,3.2 \mathrm{~Hz}, 1 \mathrm{H}), 1.30$ (qd, $J=12.2,4.4 \mathrm{~Hz}, 2 \mathrm{H})$.

${ }^{13} \mathrm{C} \mathrm{NMR}\left(126 \mathrm{MHz}, \mathrm{CDCl}_{3}\right) \delta 162.27,149.31,136.38,122.69,121.03,68.12,37.02,35.35,34.86$, 33.11 .

HRMS(EI): Calcd. For $\mathrm{C}_{12} \mathrm{H}_{17} \mathrm{NO}[\mathrm{M}]^{+}$191.1310. Found: 191.1310 .

\section{( \pm )-2-(4,4-Diethoxy-3-methylbutyl)pyridine (46)}




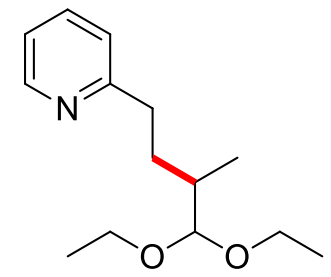

2-Vinylpyridine (21 mg, $0.2 \mathrm{mmol}$ ), 2-bromo-1,1-diethoxypropane (126.7 mg, $0.6 \mathrm{mmol}$ ), $\mathrm{Fe}\left(\mathrm{NH}_{4}\right)_{2}\left(\mathrm{SO}_{4}\right)_{2} \cdot 6 \mathrm{H}_{2} \mathrm{O}(1.6 \mathrm{mg}, 0.004 \mathrm{mmol}), 3,4,7,8$-tetramethyl-1,10-phenanthroline (1 $\mathrm{mg}, 0.004$ mmol), Zn dust (78 mg, $1.2 \mathrm{mmol})$ and NaOAc (8.2 mg, $0.1 \mathrm{mmol})$, in $0.4 \mathrm{~mL} 2 \mathrm{wt} \% \mathrm{TPGS}-750-\mathrm{M} / \mathrm{H}_{2} \mathrm{O}$ with $\mathrm{MeCN}(0.04 \mathrm{~mL})$ as co-solvent were reacted at $45^{\circ} \mathrm{C}$ for $16 \mathrm{~h}$ yielding $36.2 \mathrm{mg}(76 \%)$ of $( \pm)-2-(4,4-$ diethoxy-3-methylbutyl)pyridine as a colorless oil (hexane/EtOAc: 80/20).

${ }^{1} \mathrm{H}$ NMR $\left(500 \mathrm{MHz}, \mathrm{CDCl}_{3}\right) \delta 8.45(\mathrm{~d}, J=4.0 \mathrm{~Hz}, 1 \mathrm{H}), 7.51(\mathrm{td}, J=7.6,1.6 \mathrm{~Hz}, 1 \mathrm{H}), 7.10(\mathrm{~d}, J=$ $7.8 \mathrm{~Hz}, 1 \mathrm{H}), 7.01(\mathrm{dd}, J=7.3,5.0 \mathrm{~Hz}, 1 \mathrm{H}), 4.16(\mathrm{~d}, J=6.1 \mathrm{~Hz}, 1 \mathrm{H}), 3.66-3.52(\mathrm{~m}, 2 \mathrm{H}), 3.48-3.35(\mathrm{~m}$, 2H), $2.84(\mathrm{ddd}, J=13.7,10.7,5.4 \mathrm{~Hz}, 1 \mathrm{H}), 2.69(\mathrm{ddd}, J=13.7,10.4,6.3 \mathrm{~Hz}, 1 \mathrm{H}), 1.97-1.87(\mathrm{~m}, 1 \mathrm{H})$, $1.79-1.69(\mathrm{~m}, 1 \mathrm{H}), 1.56-1.43(\mathrm{~m}, 1 \mathrm{H}), 1.13(\mathrm{td}, J=7.0,1.6 \mathrm{~Hz}, 6 \mathrm{H}), 0.94(\mathrm{~d}, J=6.8 \mathrm{~Hz}, 3 \mathrm{H})$.

${ }^{13} \mathrm{C}$ NMR $\left(126 \mathrm{MHz}, \mathrm{CDCl}_{3}\right) \delta 162.52,149.18,136.22,122.65,120.87,106.85,62.28,62.26$, $36.32,35.98,32.10,15.35,14.47$.

HRMS(ESI): Calcd. For $\mathrm{C}_{14} \mathrm{H}_{23} \mathrm{NO}_{2} \mathrm{Na}[\mathrm{M}+\mathrm{Na}]^{+}$260.1627. Found: 260.1626 .

\section{t-Butyl 4-(2-(pyridin-2-yl)ethyl)piperidine-1-carboxylate (47)}

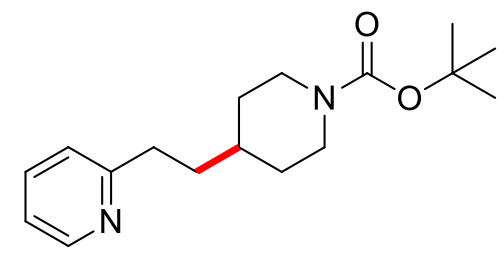

2-Vinylpyridine (21 mg, $0.2 \mathrm{mmol}$ ), 1-N-Boc-4-bromopiperidine (158.5 mg, $0.6 \mathrm{mmol}$ ), $\mathrm{Fe}\left(\mathrm{NH}_{4}\right)_{2}\left(\mathrm{SO}_{4}\right)_{2} \cdot 6 \mathrm{H}_{2} \mathrm{O}(1.6 \mathrm{mg}, 0.004 \mathrm{mmol})$, 3,4,7,8-tetramethyl-1,10-phenanthroline (1 mg, 0.004 mmol), Zn dust (78 mg, $1.2 \mathrm{mmol}$ ) and NaOAc ( $8.2 \mathrm{mg}, 0.1 \mathrm{mmol})$, in $0.4 \mathrm{~mL} 2 \mathrm{wt} \% \mathrm{TPGS}-750-\mathrm{M} / \mathrm{H}_{2} \mathrm{O}$ with $\mathrm{MeCN}(0.04 \mathrm{~mL})$ as co-solvent were reacted at $\mathrm{rt}$ for $16 \mathrm{~h}$ yielding $41 \mathrm{mg}(71 \%)$ of tert-butyl 4-(2(pyridin-2-yl)ethyl)piperidine-1-carboxylate as a yellow oil (hexane/EtOAc: 70/30).

${ }^{1} \mathrm{H}$ NMR $\left(500 \mathrm{MHz}, \mathrm{CDCl}_{3}\right) \delta 8.42(\mathrm{~d}, J=3.0 \mathrm{~Hz}, 1 \mathrm{H}), 7.48(\mathrm{td}, J=7.6,1.9 \mathrm{~Hz}, 1 \mathrm{H}), 7.04(\mathrm{~d}, J=$ $7.8 \mathrm{~Hz}, 1 \mathrm{H}), 6.99(\mathrm{dd}, J=6.9,4.3 \mathrm{~Hz}, 1 \mathrm{H}), 3.99(\mathrm{br}, 2 \mathrm{H}), 2.75-2.68$ (m, 2H), $2.58(\mathrm{br}, 2 \mathrm{H}), 1.68-1.54$ 
(m, 4H), $1.36(\mathrm{~s}, 10 \mathrm{H}), 1.10-1.00(\mathrm{~m}, 2 \mathrm{H})$.

${ }^{13} \mathrm{C}$ NMR $\left(126 \mathrm{MHz}, \mathrm{CDCl}_{3}\right) \delta 162.07,154.78,149.16,136.24,122.56,120.90,79.05,43.95(\mathrm{br})$, $36.46,35.72,35.44,32.02$ (br), 28.42.

HRMS(ESI): Calcd. For $\mathrm{C}_{17} \mathrm{H}_{27} \mathrm{~N}_{2} \mathrm{O}_{2}[\mathrm{M}+\mathrm{H}]^{+}$291.2072. Found: 291.2068 .

\section{3-Methyl-5-(pyridin-2-yl)pentanenitrile (48)}

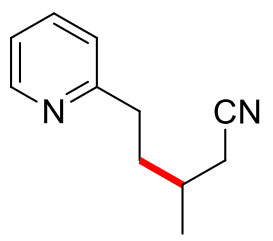

2-Vinylpyridine (21 $\mathrm{mg}, \quad 0.2 \mathrm{mmol}), \quad$ 3-bromobutanenitrile $(88.8 \mathrm{mg}, \quad 0.6 \mathrm{mmol})$, $\mathrm{Fe}\left(\mathrm{NH}_{4}\right)_{2}\left(\mathrm{SO}_{4}\right)_{2} \cdot 6 \mathrm{H}_{2} \mathrm{O}$ (1.6 mg, $\left.0.004 \mathrm{mmol}\right), 3,4,7,8$-tetramethyl-1,10-phenanthroline $(1 \mathrm{mg}, 0.004$ $\mathrm{mmol}$ ), $\mathrm{Zn}$ dust (78 mg, $1.2 \mathrm{mmol}$ ) and $\mathrm{NaOAc}(8.2 \mathrm{mg}, 0.1 \mathrm{mmol})$, in $0.4 \mathrm{~mL} 2 \mathrm{wt} \% \mathrm{TPGS}-750-\mathrm{M} / \mathrm{H}_{2} \mathrm{O}$ with $\mathrm{MeCN}(0.04 \mathrm{~mL})$ as co-solvent were reacted at $\mathrm{rt}$ for $16 \mathrm{~h}$ yielding $21.5 \mathrm{mg}(62 \%)$ of 3-methyl-5(pyridin-2-yl)pentanenitrile as a yellow oil (hexane/EtOAc: 70/30).

${ }^{1} \mathrm{H}$ NMR $\left(500 \mathrm{MHz}, \mathrm{CDCl}_{3}\right) \delta 8.47(\mathrm{~d}, J=4.3 \mathrm{~Hz}, 1 \mathrm{H}), 7.63-7.46(\mathrm{~m}, 1 \mathrm{H}), 7.19-6.97(\mathrm{~m}, 2 \mathrm{H})$, $2.86-2.68$ (m, 2H), 2.30 (qd, $J=16.7,5.9 \mathrm{~Hz}, 2 \mathrm{H}), 1.93-1.78$ (m, 2H), $1.75-1.64$ (m, 1H), 1.09 (d, $J$ $=6.5 \mathrm{~Hz}, 3 \mathrm{H})$.

${ }^{13} \mathrm{C}$ NMR $\left(126 \mathrm{MHz}, \mathrm{CDCl}_{3}\right) \delta 161.08,149.29,136.55,122.74,121.29,118.74,35.76,35.51$, $30.11,24.44,19.34$.

HRMS(ESI): Calcd. For $\mathrm{C}_{11} \mathrm{H}_{14} \mathrm{~N}_{2} \mathrm{Na}[\mathrm{M}+\mathrm{Na}]^{+}$197.1051. Found: 197.1055 .

\section{2-(2-(Adamantan-1-yl)ethyl)pyridine (49)}

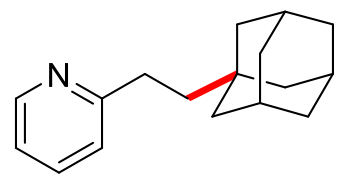

2-Vinylpyridine $(21 \mathrm{mg}, \quad 0.2 \mathrm{mmol}), \quad$ 1-bromoadamantane $(129.1 \mathrm{mg}, \quad 0.6 \mathrm{mmol})$, $\mathrm{Fe}\left(\mathrm{NH}_{4}\right)_{2}\left(\mathrm{SO}_{4}\right)_{2} \cdot 6 \mathrm{H}_{2} \mathrm{O}$ (1.6 mg, $\left.0.004 \mathrm{mmol}\right), 3,4,7,8$-tetramethyl-1,10-phenanthroline $(1 \mathrm{mg}, 0.004$ $\mathrm{mmol}$ ), Zn dust (78 mg, $1.2 \mathrm{mmol}$ ) and NaOAc ( $8.2 \mathrm{mg}, 0.1 \mathrm{mmol}$ ), in $0.4 \mathrm{~mL} 2 \mathrm{wt} \% \mathrm{TPGS}-750-\mathrm{M} / \mathrm{H}_{2} \mathrm{O}$ 
with $\mathrm{MeCN}(0.04 \mathrm{~mL})$ as co-solvent were reacted at $45{ }^{\circ} \mathrm{C}$ for $16 \mathrm{~h}$ yielding $30.4 \mathrm{mg}(63 \%)$ of 2-(2(adamantan-1-yl)ethyl)pyridine as a colorless oil (hexane/EtOAc: 80/20).

${ }^{1} \mathrm{H}$ NMR $\left(500 \mathrm{MHz}, \mathrm{CDCl}_{3}\right) \delta 8.51(\mathrm{~d}, J=5.7 \mathrm{~Hz}, 1 \mathrm{H}), 7.57(\mathrm{td}, J=7.7,1.8 \mathrm{~Hz}, 1 \mathrm{H}), 7.14(\mathrm{~d}, J$ $=7.8 \mathrm{~Hz}, 1 \mathrm{H}), 7.11-7.04(\mathrm{~m}, 1 \mathrm{H}), 2.78-2.69(\mathrm{~m}, 2 \mathrm{H}), 1.97(\mathrm{~s}, 3 \mathrm{H}), 1.72(\mathrm{~d}, J=12.0 \mathrm{~Hz}, 3 \mathrm{H}), 1.65(\mathrm{~d}$, $J=11.2 \mathrm{~Hz}, 3 \mathrm{H}), 1.56(\mathrm{~d}, J=2.4 \mathrm{~Hz}, 6 \mathrm{H}), 1.51-1.45(\mathrm{~m}, 2 \mathrm{H})$.

${ }^{13} \mathrm{C} \mathrm{NMR}\left(126 \mathrm{MHz}, \mathrm{CDCl}_{3}\right) \delta 163.62,149.31,136.39,122.78,120.85,44.93,42.55,37.38,32.60$, $32.00,28.92$.

HRMS(ESI): Calcd. For $\mathrm{C}_{17} \mathrm{H}_{24} \mathrm{~N}[\mathrm{M}+\mathrm{H}]^{+}$242.1909. Found: 242.1900 .

\section{2-(3,3-Dimethylbutyl)pyridine (50)}

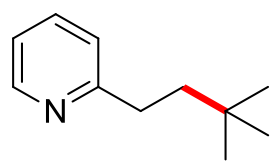

2-Vinylpyridine (21 mg, $0.2 \mathrm{mmol}$ ), 2-bromo-2-methylpropane (82.2 $\mathrm{mg}, \quad 0.6 \mathrm{mmol}$ ), $\mathrm{Fe}\left(\mathrm{NH}_{4}\right)_{2}\left(\mathrm{SO}_{4}\right)_{2} \cdot 6 \mathrm{H}_{2} \mathrm{O}(1.6 \mathrm{mg}, 0.004 \mathrm{mmol}), 3,4,7,8$-tetramethyl-1,10-phenanthroline (1 mg, 0.004 $\mathrm{mmol}$ ), $\mathrm{Zn}$ dust (78 mg, $1.2 \mathrm{mmol}$ ) and NaOAc ( $8.2 \mathrm{mg}, 0.1 \mathrm{mmol}$ ), in $0.4 \mathrm{~mL} 2 \mathrm{wt} \% \mathrm{TPGS}-750-\mathrm{M} / \mathrm{H}_{2} \mathrm{O}$ with $\mathrm{MeCN}(0.04 \mathrm{~mL})$ as co-solvent were reacted at $45{ }^{\circ} \mathrm{C}$ for $16 \mathrm{~h}$ yielding $20.2 \mathrm{mg}(62 \%)$ of 2-(3,3dimethylbutyl)pyridine as a colorless oil (hexane/EtOAc: 80/20).

Spectral data matched that in the reported literature. ${ }^{10}{ }^{1} \mathrm{H}$ NMR $\left(500 \mathrm{MHz}, \mathrm{CDCl}_{3}\right) \delta 8.51(\mathrm{~d}, J=$ $4.8 \mathrm{~Hz}, 1 \mathrm{H}), 7.57$ (td, $J=7.6,1.4 \mathrm{~Hz}, 1 \mathrm{H}), 7.14$ (d, $J=7.8 \mathrm{~Hz}, 1 \mathrm{H}), 7.07$ (dd, $J=7.3,5.0 \mathrm{~Hz}, 1 \mathrm{H}), 2.79-$ $2.72(\mathrm{~m}, 2 \mathrm{H}), 1.64-1.58(\mathrm{~m}, 2 \mathrm{H}), 0.97(\mathrm{~s}, 9 \mathrm{H})$.

${ }^{13} \mathrm{C} \mathrm{NMR}\left(126 \mathrm{MHz}, \mathrm{CDCl}_{3}\right) \delta 163.32,149.33,136.41,122.72,120.90,44.46,34.07,30.47,29.48$.

\section{References}

1. Lipshutz, B. H.; Ghorai, S.; Abela, A. R.; Moser, R.; Nishikata, T.; Duplais, C.; Krasovskiy, A.; Gaston, R. D.; Gadwood, R. C. J. Org. Chem. 2011, 76, 4379.

2. Gottlieb, H. E.; Kotlyar, V.; Nudelman, A. J. Org. Chem. 1997, 62, 7512. 
3. Lipshutz, B. H.; Petersen, T. B.; Abela, A. R. Org. Lett. 2008, 10, 1333.

4. Zhang, J.-Z.; Tang, Y. Adv. Synth. Catal. 2016, 358, 752.

5. $\quad$ Dick, G. R.; Knapp, D. M.; Gillis, E. P.; Burke, M. D. Org. Lett. 2010, 12, 2314.

6. Isley, N. A.; Wang, Y.; Gallou, F.; Handa, S.; Aue, D. H.; Lipshutz, B. H. ACS Catal. 2017, 7, 8331.

7. Strauch, R. C.; Mastarone, D. J.; Sukerkar, P. A.; Song, Y.; Ipsaro, J. J.; Meade, T. J. J. Am. Chem. Soc. 2011, 133, 16346.

8. $\quad$ Kristensen, S. K.; Laursen, S. L. R.; Taarning, E.; Skrydstrup, T., Angew. Chem. Int. Ed. 2018, $57,13887$.

9. $\quad$ Lipshutz, B. H.; Huang, S.; Leong, W. W. Y.; Zhong, G.; Isley, N. A., J. Am. Chem. Soc. 2012, 134, 19985.

10. Lewis, J. C.; Bergman, R. G.; Ellman, J. A. J. Am. Chem. Soc. 2007, 129, 5332. 


\section{2. ${ }^{1} \mathrm{H}$ NMR and ${ }^{13} \mathrm{C}$ NMR spectra}

${ }^{1} \mathrm{H} \mathrm{NMR}\left(500 \mathrm{MHz}, \mathrm{CDCl}_{3}\right),{ }^{13} \mathrm{C} \mathrm{NMR}\left(126 \mathrm{MHz}, \mathrm{CDCl}_{3}\right)$<smiles>CC(C)(C)c1ccc(CCCCCOc2ccccc2)cc1</smiles>

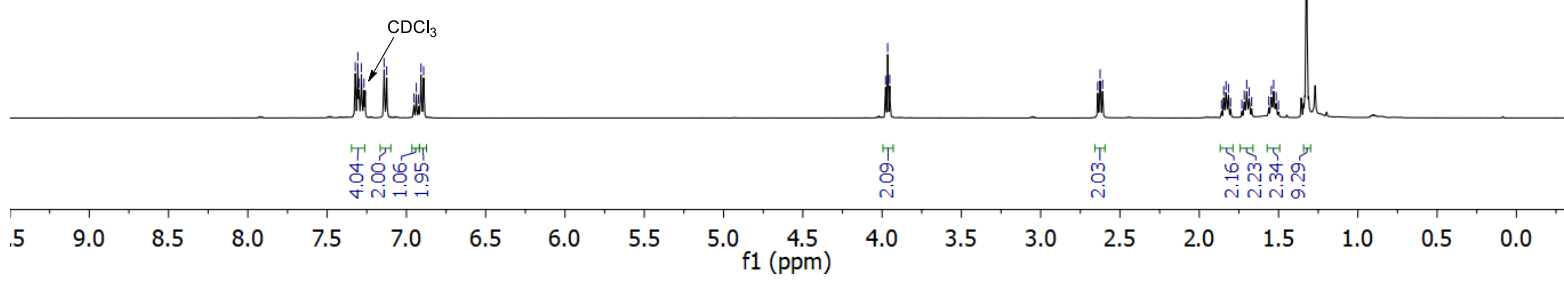

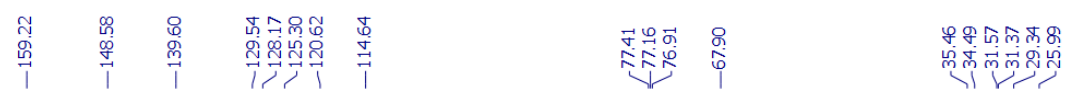<smiles>CC(C)(C)c1ccc(CCCCCOc2ccccc2)cc1</smiles>

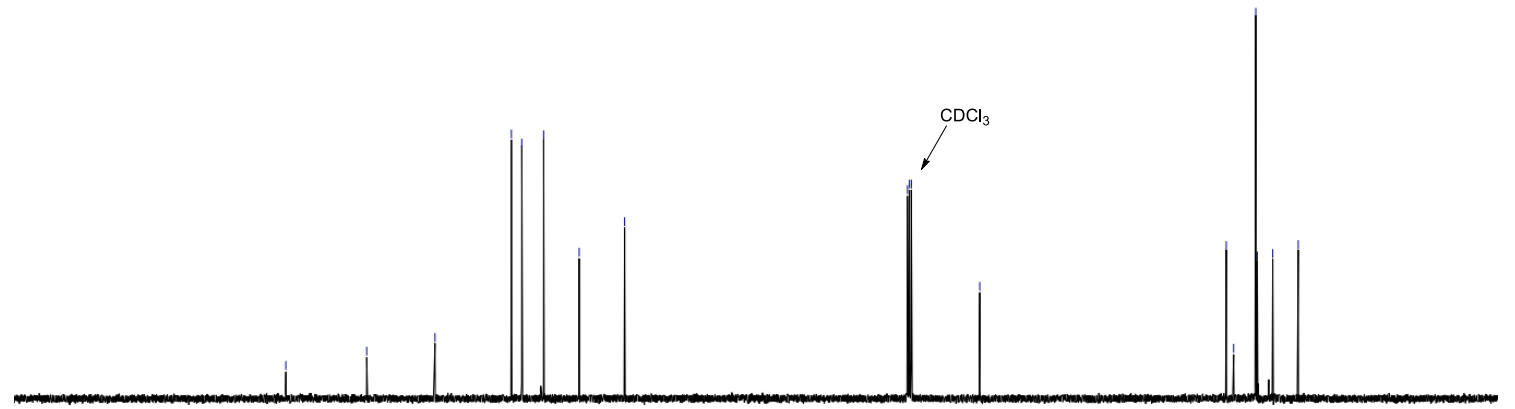

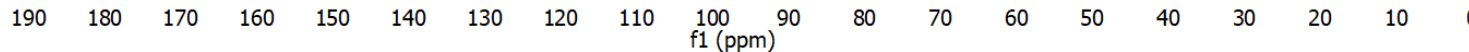


${ }^{1} \mathrm{H}$ NMR $\left(500 \mathrm{MHz}, \mathrm{CDCl}_{3}\right),{ }^{13} \mathrm{C} \mathrm{NMR}\left(126 \mathrm{MHz}, \mathrm{CDCl}_{3}\right)$<smiles>Brc1ccc(CCCCCOc2ccccc2)cc1</smiles>

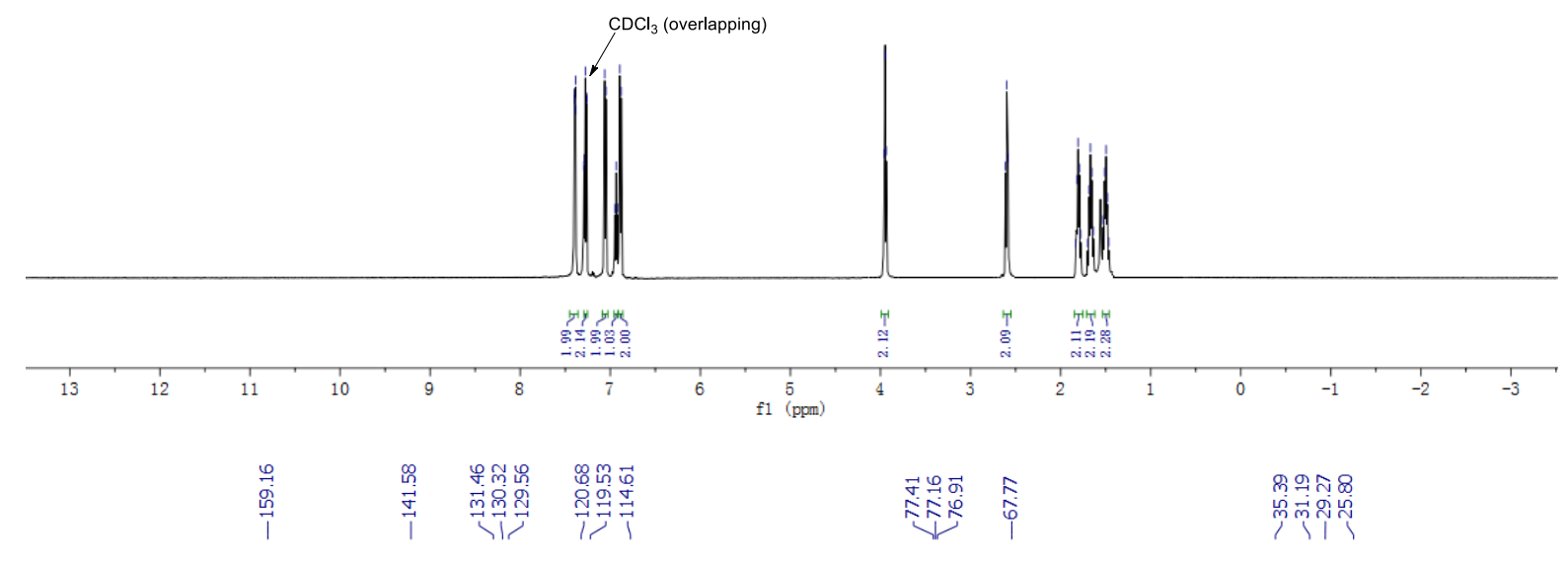<smiles>Brc1ccc(CCCCCOc2ccccc2)cc1</smiles>

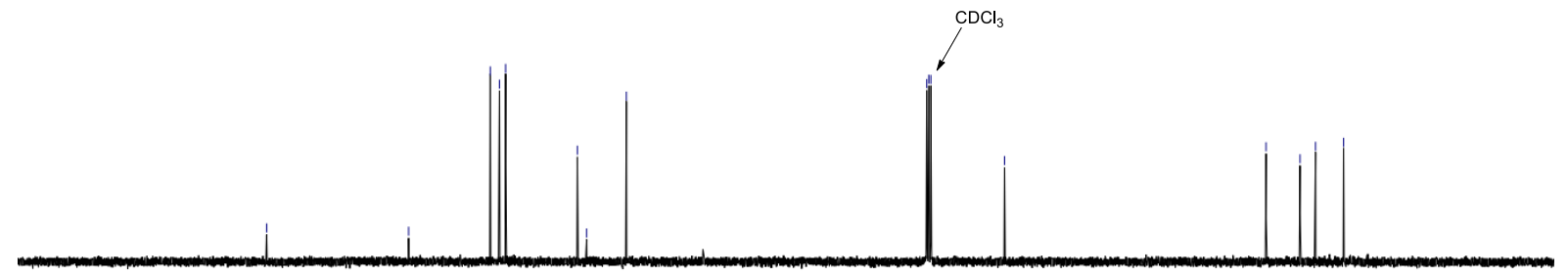

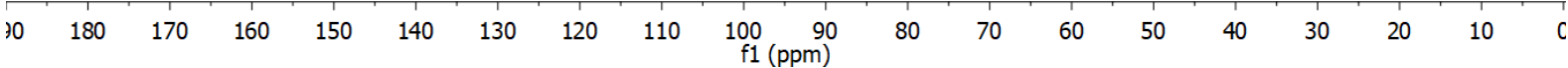


${ }^{1} \mathrm{H} \mathrm{NMR}\left(500 \mathrm{MHz}, \mathrm{CDCl}_{3}\right),{ }^{13} \mathrm{C} \mathrm{NMR}\left(126 \mathrm{MHz}, \mathrm{CDCl}_{3}\right),{ }^{19} \mathrm{~F} \mathrm{NMR}\left(376 \mathrm{MHz}, \mathrm{CDCl}_{3}\right)$<smiles>FC(F)(F)c1ccc(CCCCCOc2ccccc2)cc1</smiles>

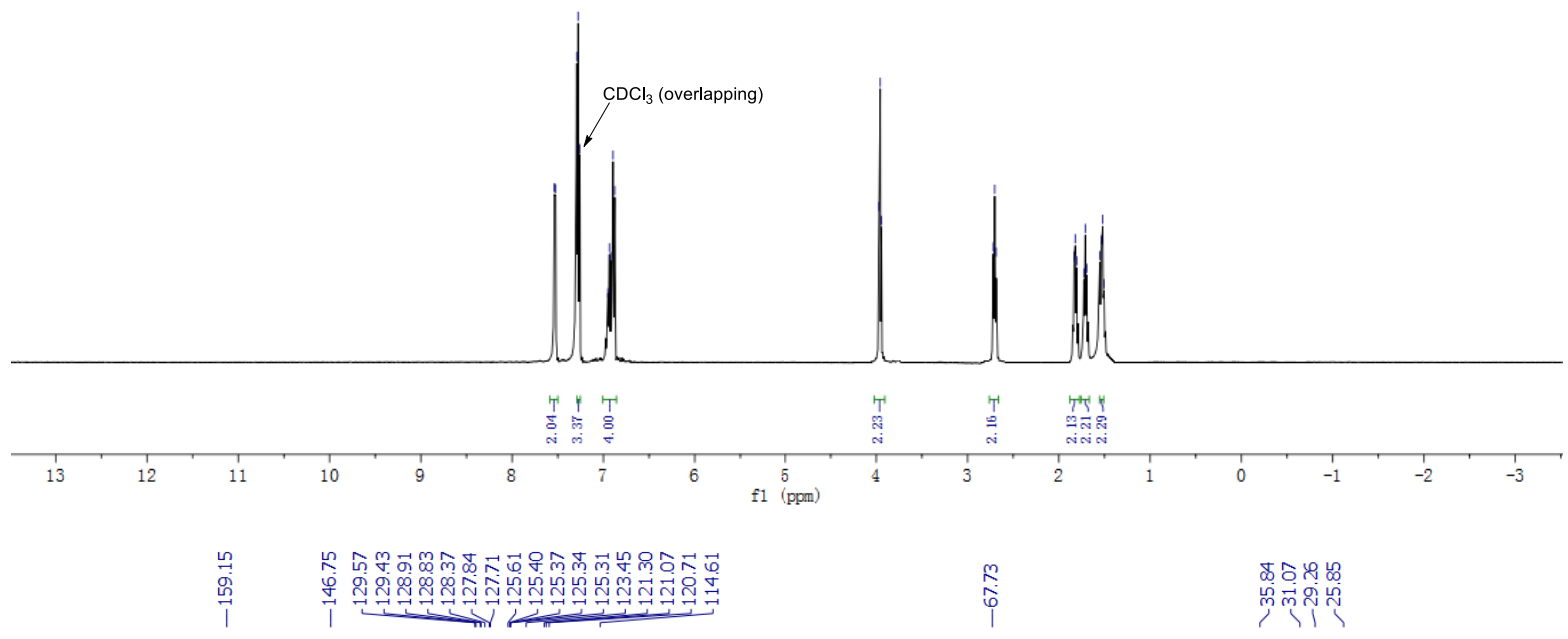<smiles>FC(F)(F)c1ccc(CCCCCOc2ccccc2)cc1</smiles>

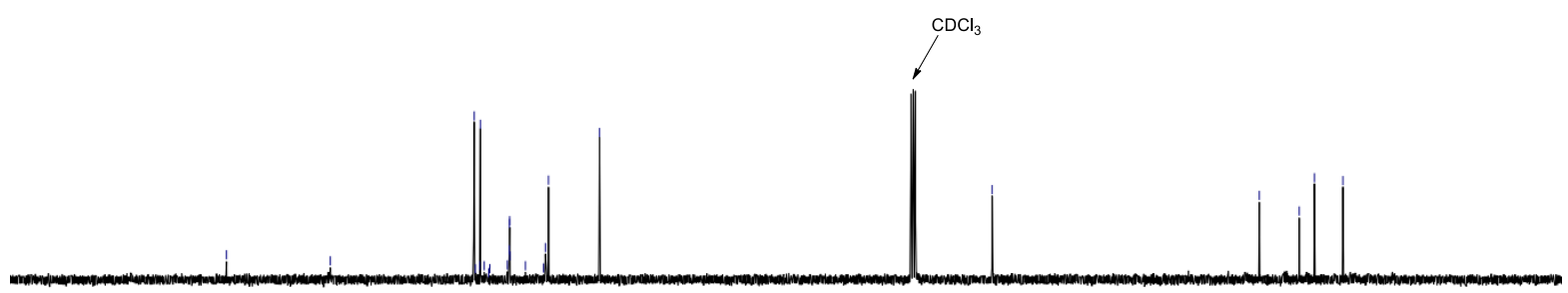

$\begin{array}{llllllllllllllllll}180 & 170 & 160 & 150 & 140 & 130 & 120 & 110 & 100 \begin{array}{c}90 \\ \mathrm{f} 1(\mathrm{ppm})\end{array} & 80 & 70 & 60 & 50 & 40 & 30 & 20 & 10 & \mathrm{C}\end{array}$


<smiles>FC(F)(F)c1ccc(CCCCCOc2ccccc2)cc1</smiles>

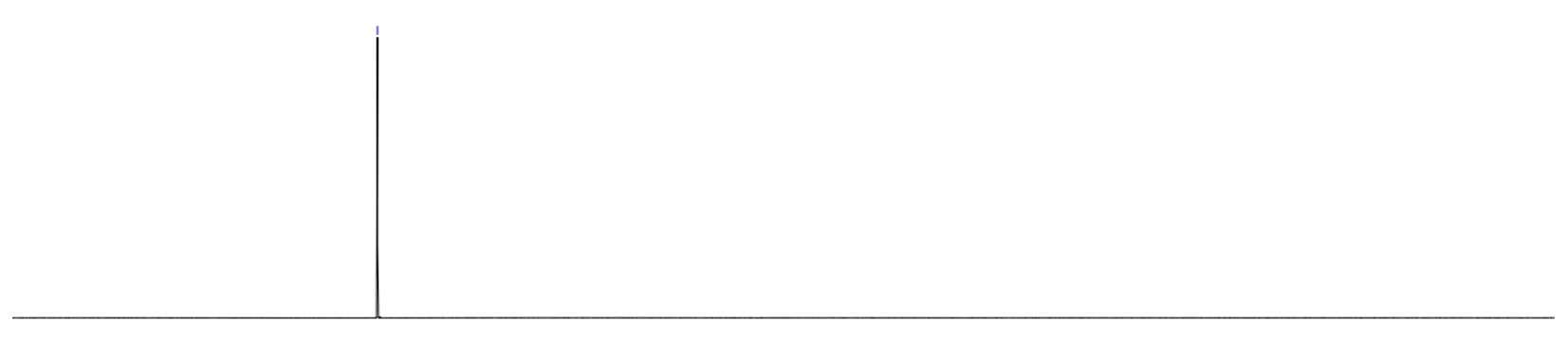

\begin{tabular}{llllllllllllllllll}
\hline 10 & -10 & -30 & -50 & -70 & -90 & -110 & -130 & -150 & -170 & -190 & -210 & -230 & -250 & -270 & -290 & -310
\end{tabular}


${ }^{1} \mathrm{H}$ NMR $\left(500 \mathrm{MHz}, \mathrm{CDCl}_{3}\right),{ }^{13} \mathrm{C}$ NMR $\left(126 \mathrm{MHz}, \mathrm{CDCl}_{3}\right)$

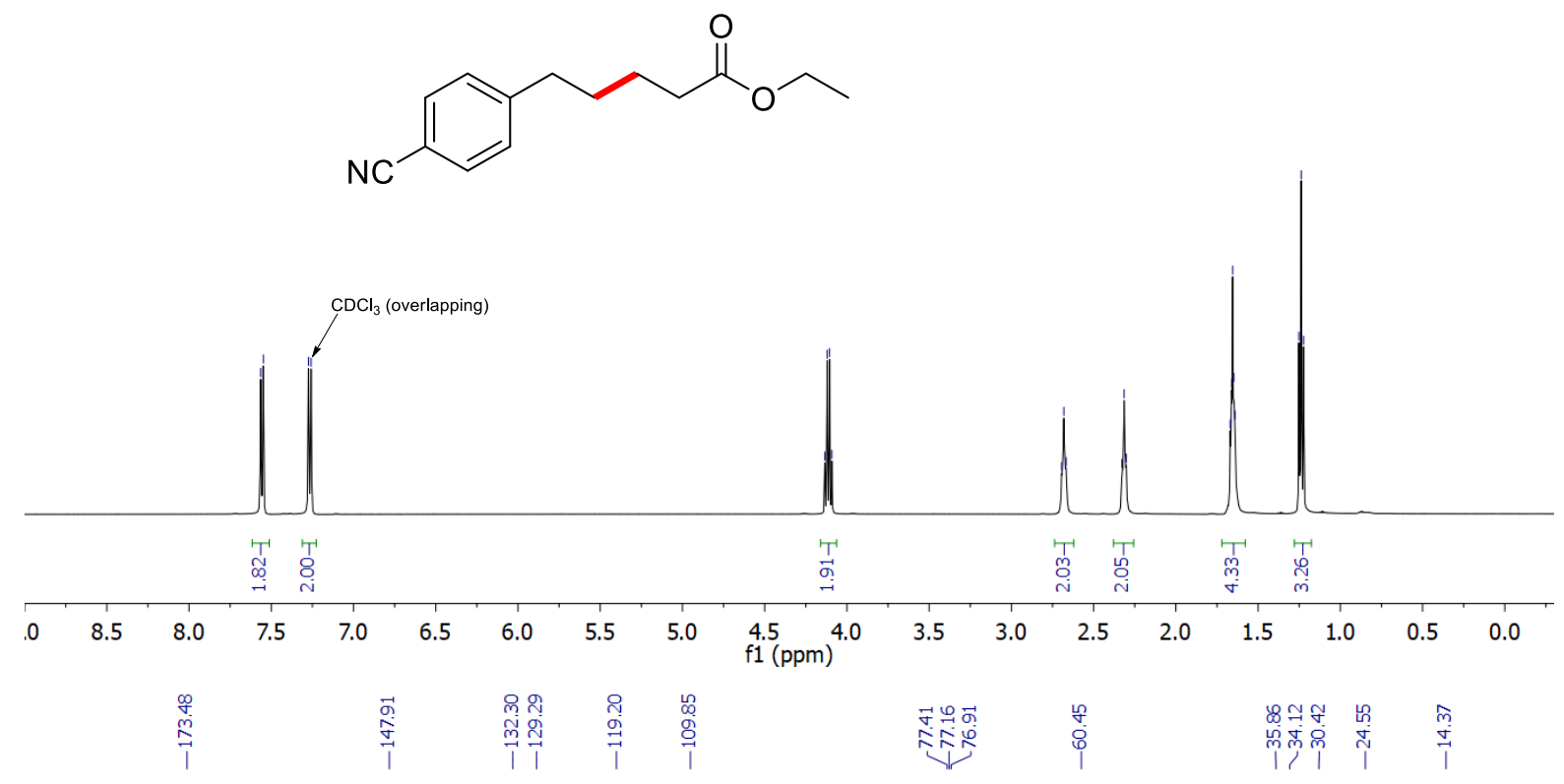<smiles>CCOC(=O)CCCCc1ccc(C#N)cc1</smiles>

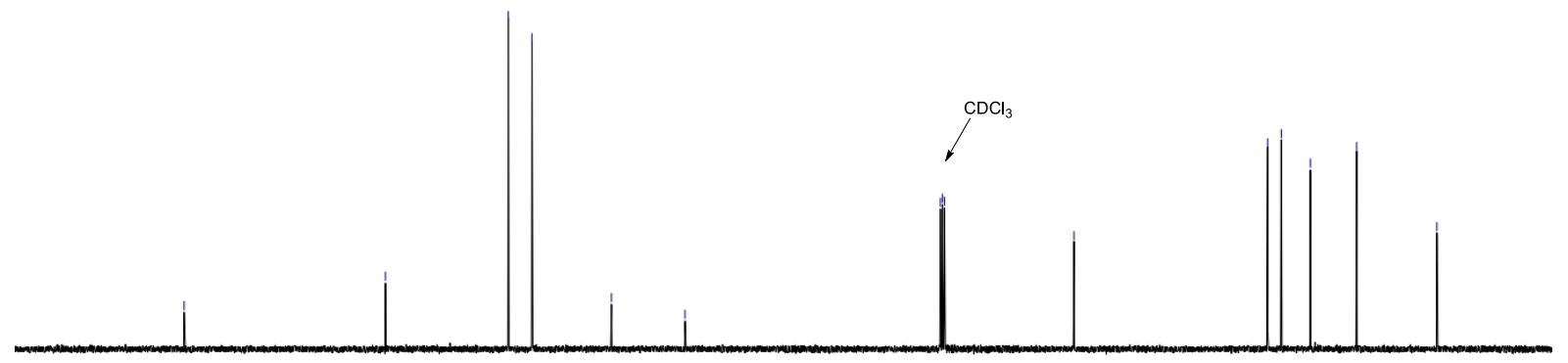


${ }^{1} \mathrm{H}$ NMR $\left(500 \mathrm{MHz}, \mathrm{CDCl}_{3}\right),{ }^{13} \mathrm{C} \mathrm{NMR}\left(126 \mathrm{MHz}, \mathrm{CDCl}_{3}\right)$

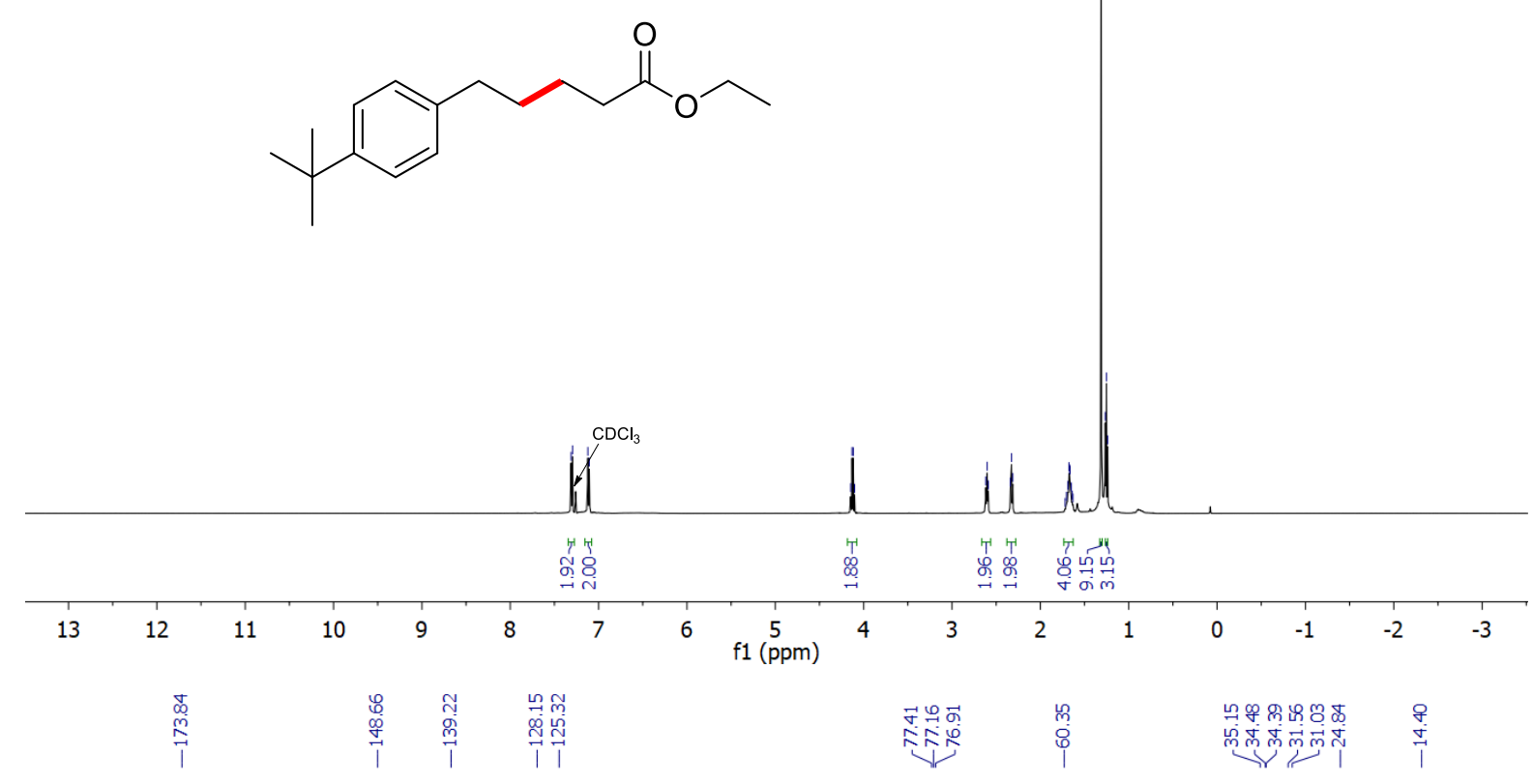<smiles>CCOC(=O)CCCCc1ccc(C(C)(C)C)cc1</smiles>

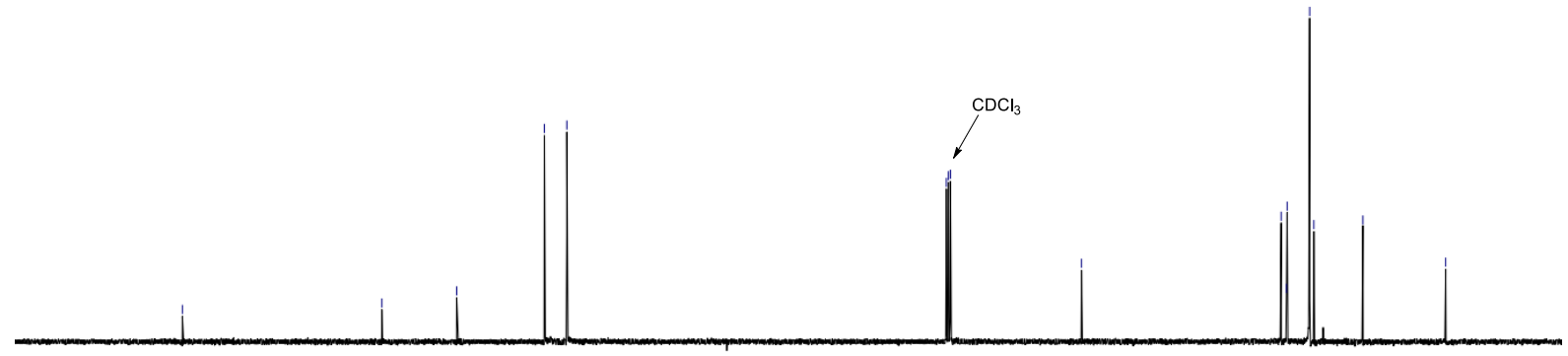

$190 \quad 180 \quad 170$

160 
${ }^{1} \mathrm{H}$ NMR $\left(500 \mathrm{MHz}, \mathrm{CDCl}_{3}\right),{ }^{13} \mathrm{C} \mathrm{NMR}\left(126 \mathrm{MHz}, \mathrm{CDCl}_{3}\right)$<smiles>CCOC(=O)CCCCc1cccc2ccccc12</smiles>
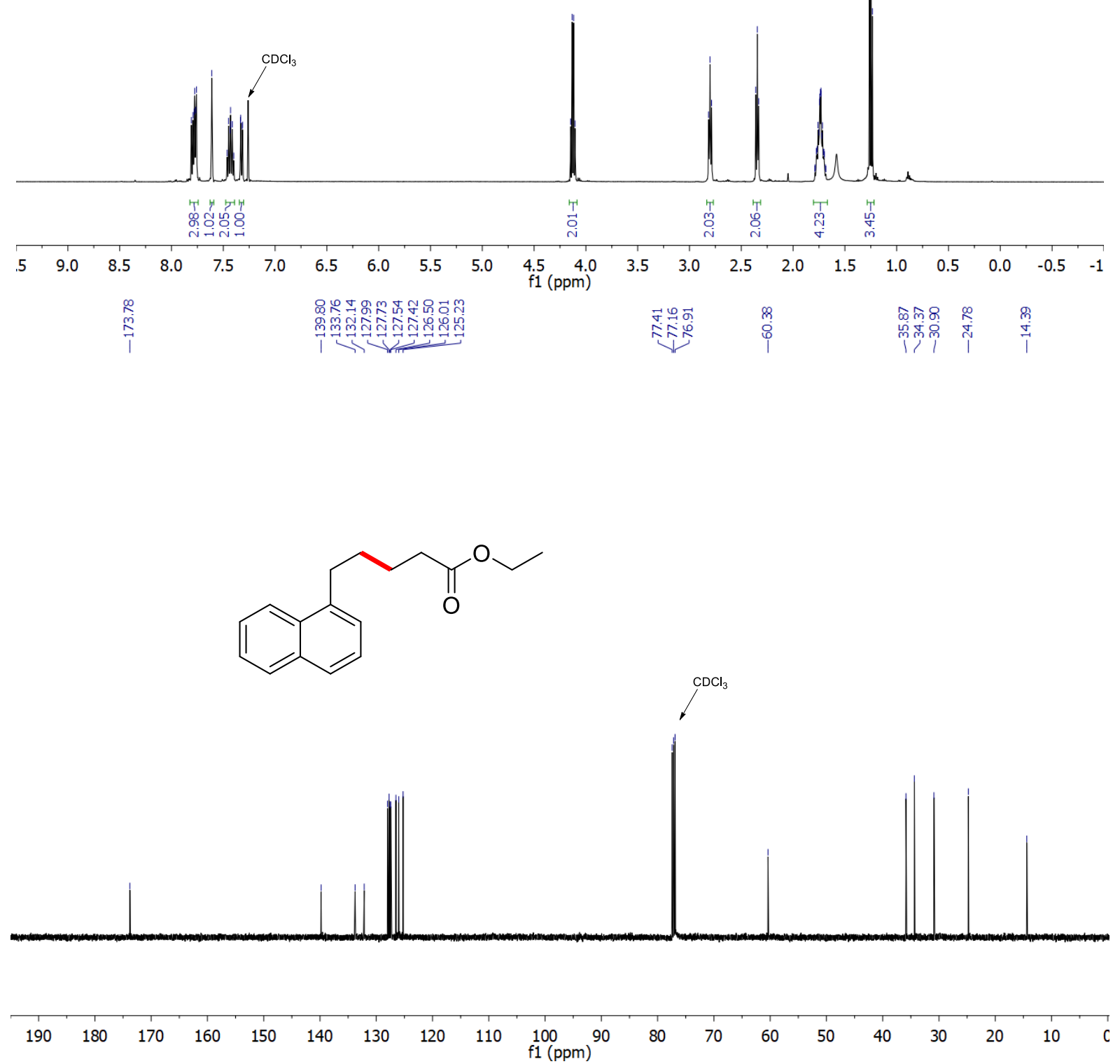
${ }^{1} \mathrm{H}$ NMR $\left(500 \mathrm{MHz}, \mathrm{CDCl}_{3}\right),{ }^{13} \mathrm{C} \mathrm{NMR}\left(126 \mathrm{MHz}, \mathrm{CDCl}_{3}\right)$<smiles>Clc1ccccc1CCCCCOc1ccccc1</smiles>
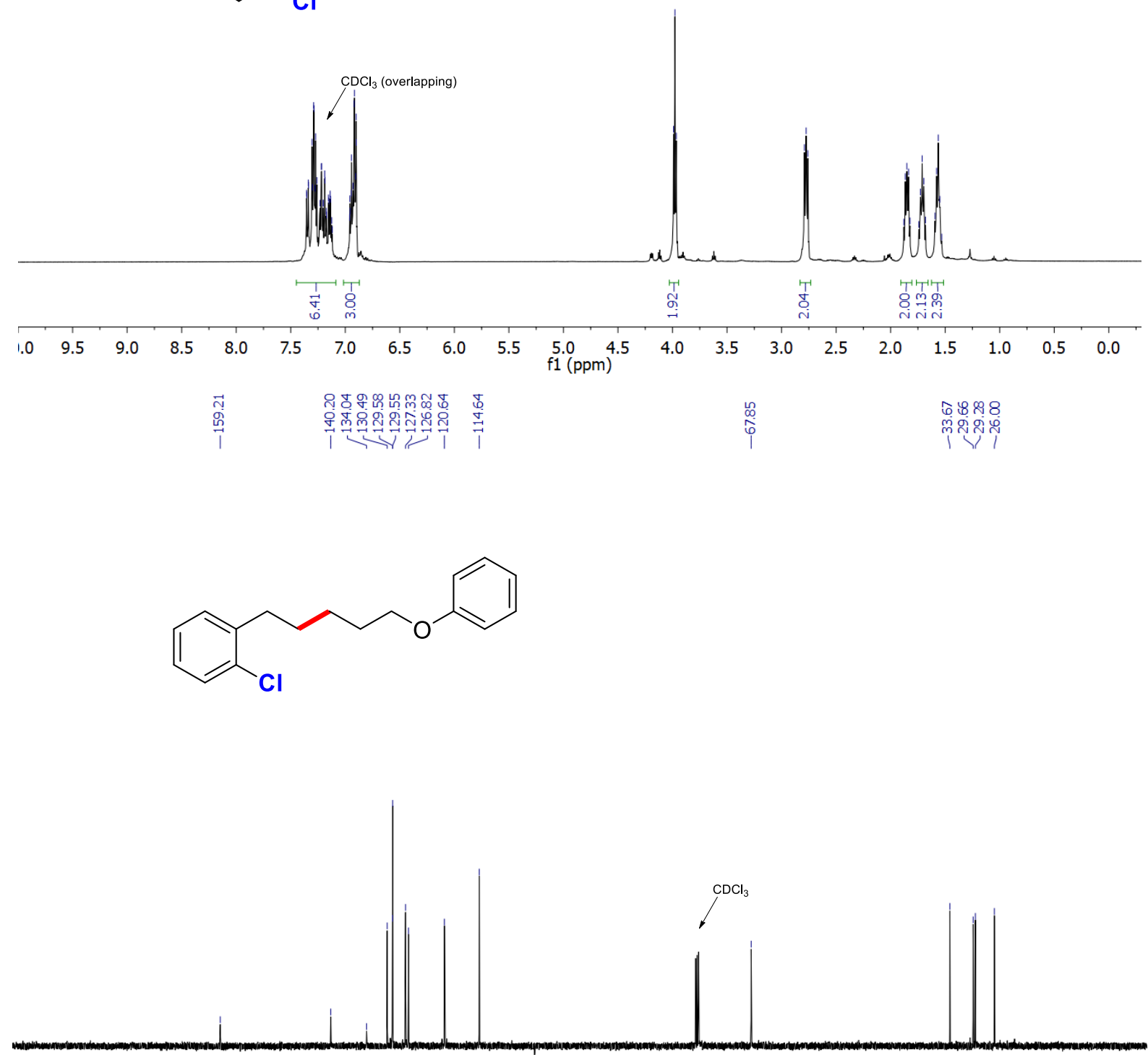

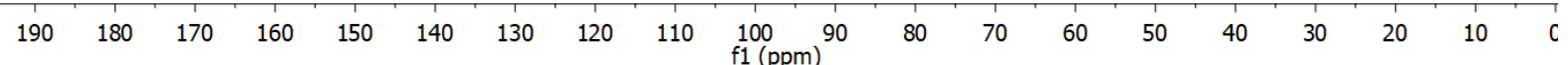




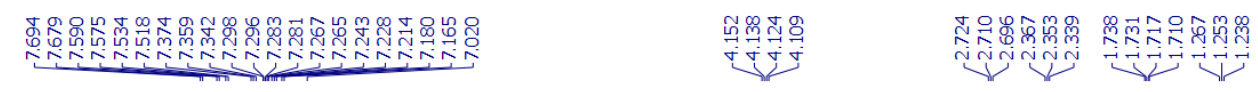

${ }^{1} \mathrm{H}$ NMR $\left(500 \mathrm{MHz}, \mathrm{CDCl}_{3}\right),{ }^{13} \mathrm{C} \mathrm{NMR}\left(126 \mathrm{MHz}, \mathrm{CDCl}_{3}\right)$<smiles>CCOC(=O)CCCCc1cccc(-c2cc3ccccc3o2)c1</smiles>

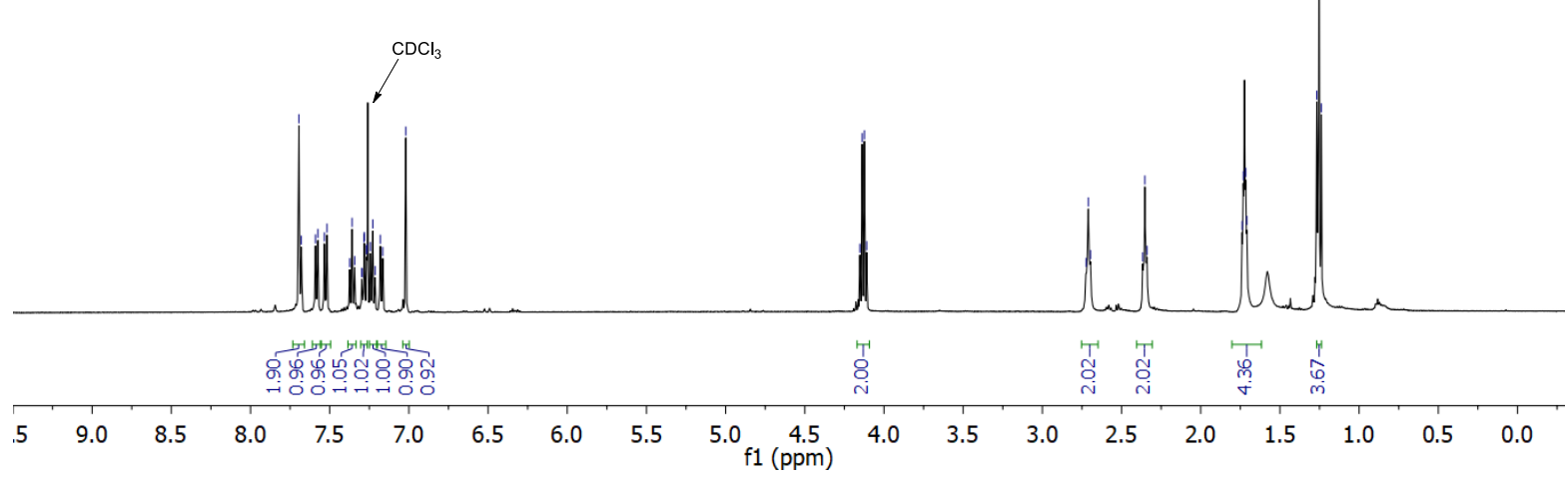

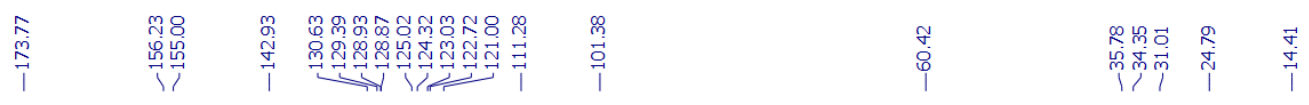
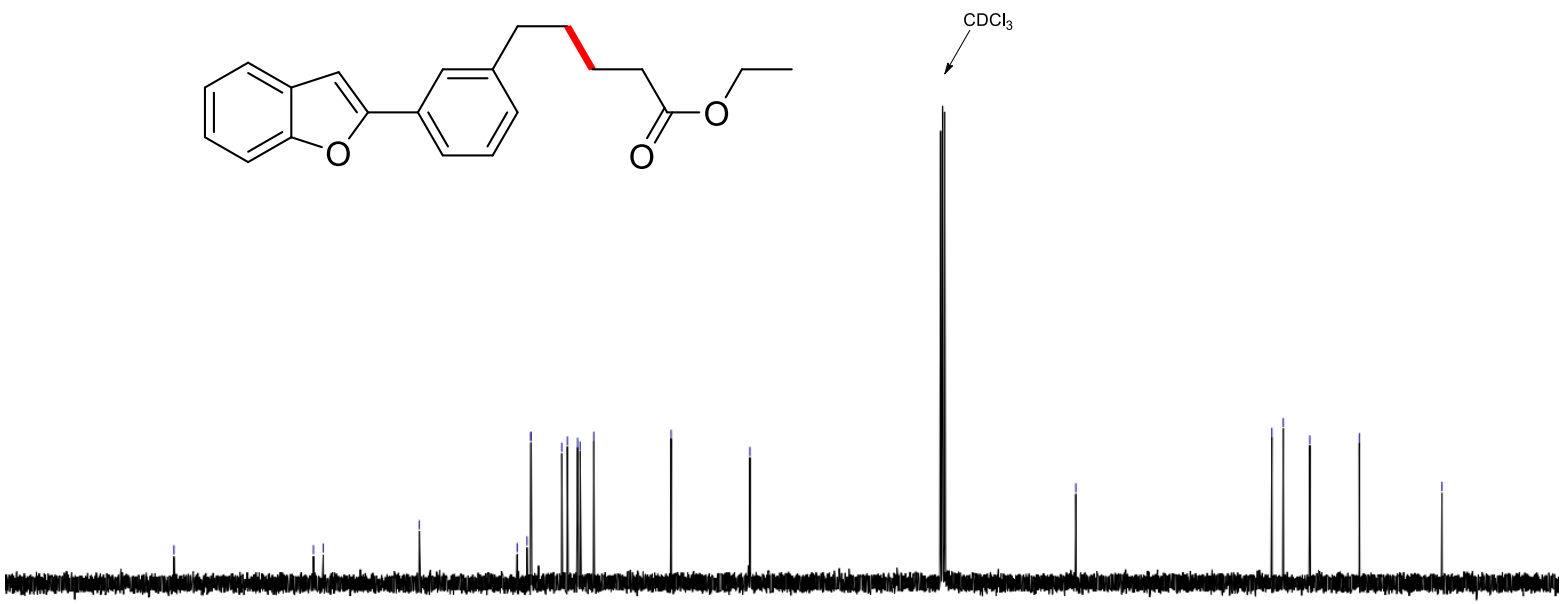

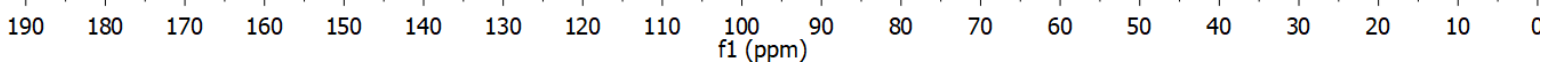


${ }^{1} \mathrm{H}$ NMR $\left(500 \mathrm{MHz}, \mathrm{CDCl}_{3}\right),{ }^{13} \mathrm{C} \mathrm{NMR}\left(126 \mathrm{MHz}, \mathrm{CDCl}_{3}\right)$

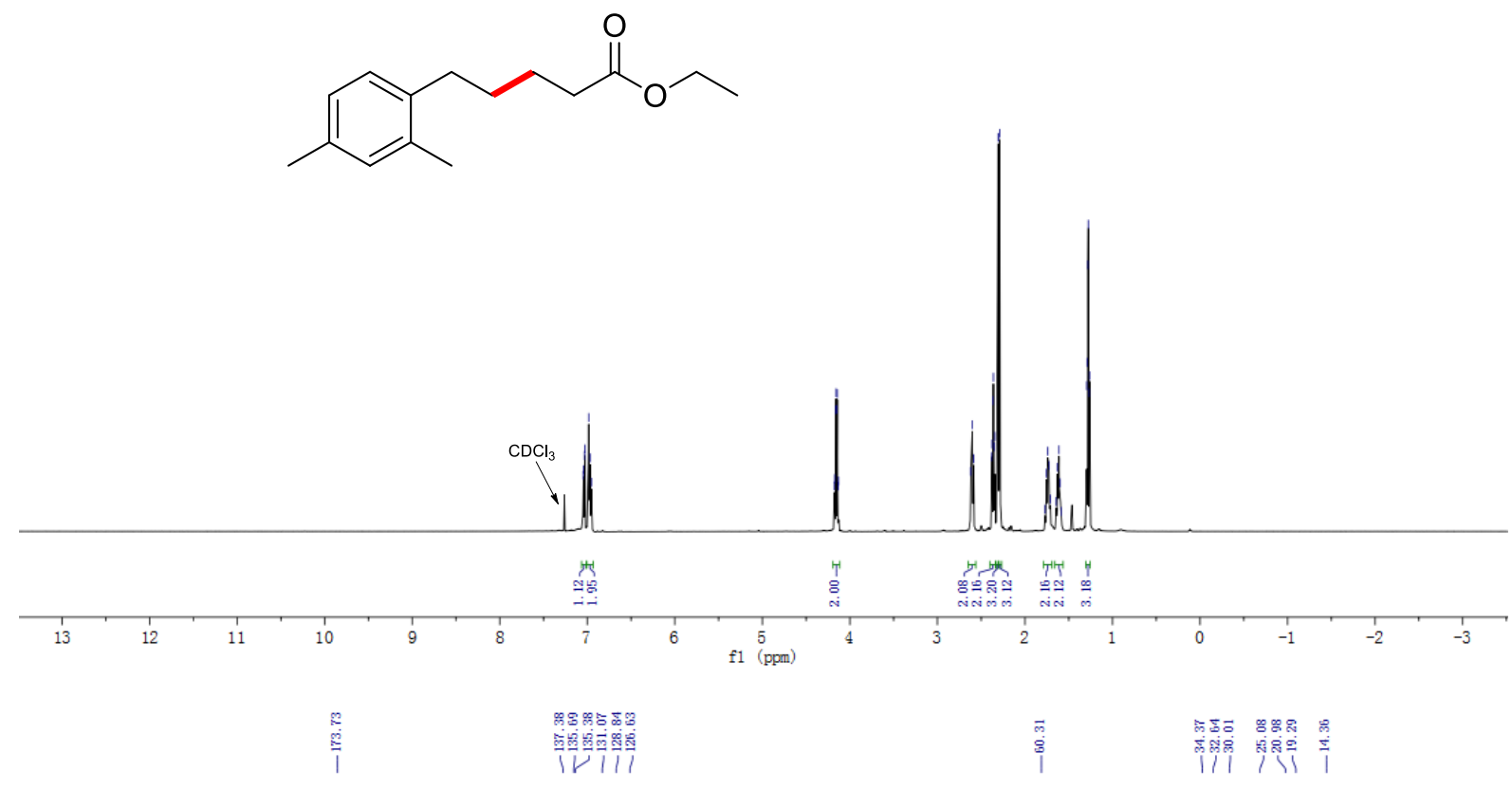<smiles>CCOC(=O)CCCCc1ccc(C)cc1C</smiles>

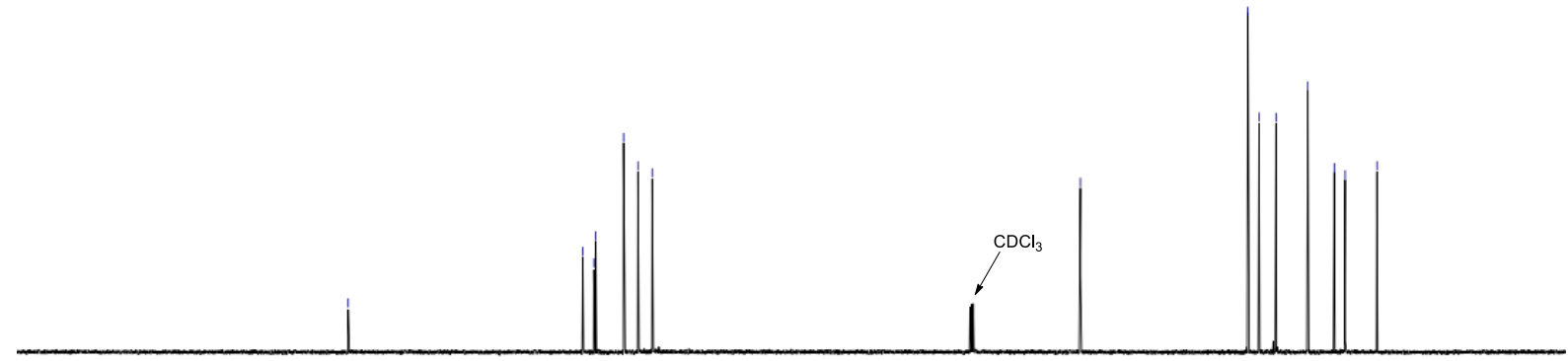

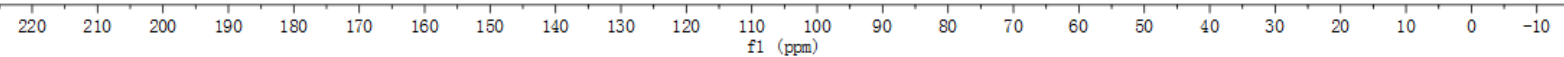


${ }^{1} \mathrm{H}$ NMR $\left(500 \mathrm{MHz}, \mathrm{CDCl}_{3}\right),{ }^{13} \mathrm{C} \mathrm{NMR}\left(126 \mathrm{MHz}, \mathrm{CDCl}_{3}\right)$

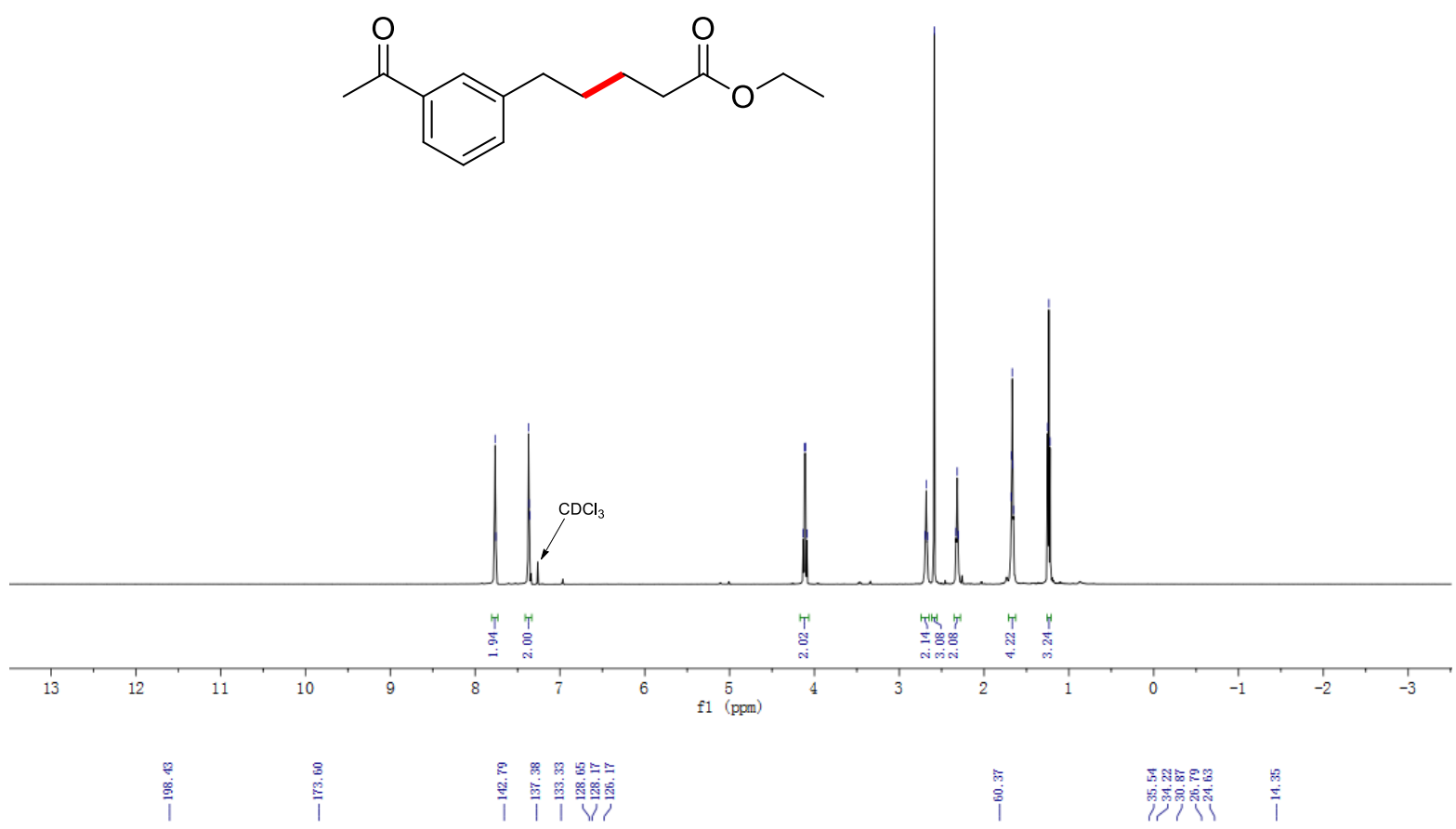<smiles>CCOC(=O)CCCCc1cccc(C(C)=O)c1</smiles>

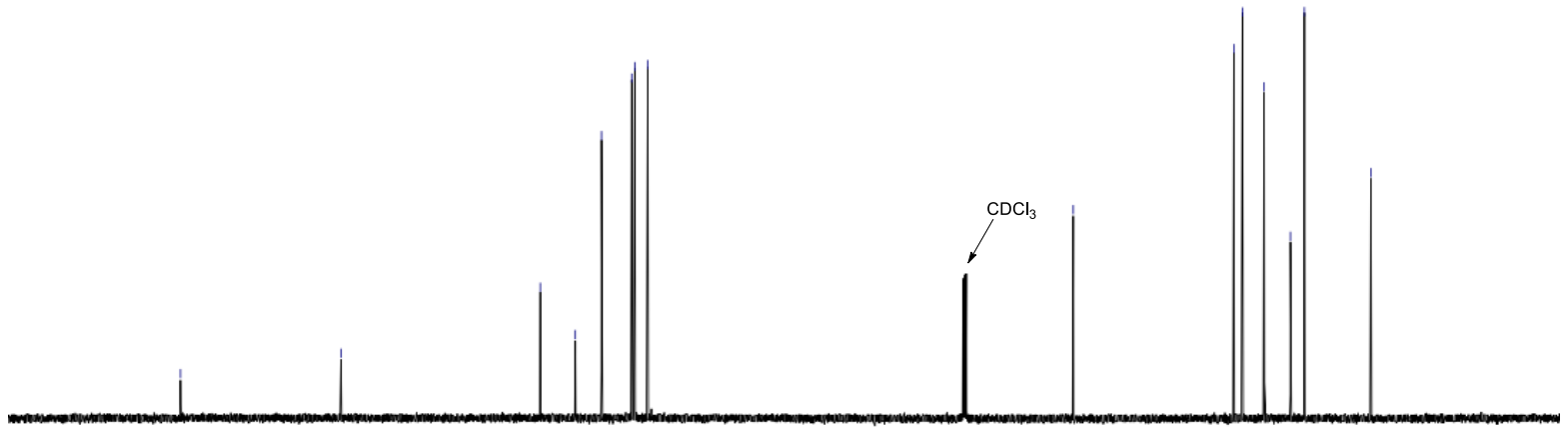

$\begin{array}{llllllllllllllllllllllll}2 & 210 & 200 & 190 & 180 & 170 & 160 & 150 & 140 & 130 & 120 & \underset{1}{1} 10 & 100 & 90 & 80 & 70 & 60 & 50 & 40 & 30 & 20 & 10 & 0 & -10\end{array}$ 
${ }^{1} \mathrm{H} \mathrm{NMR}\left(500 \mathrm{MHz}, \mathrm{CDCl}_{3}\right),{ }^{13} \mathrm{C} \mathrm{NMR}\left(126 \mathrm{MHz}, \mathrm{CDCl}_{3}\right)$<smiles>C=CCc1ccccc1CCCCC(=O)OCC</smiles>

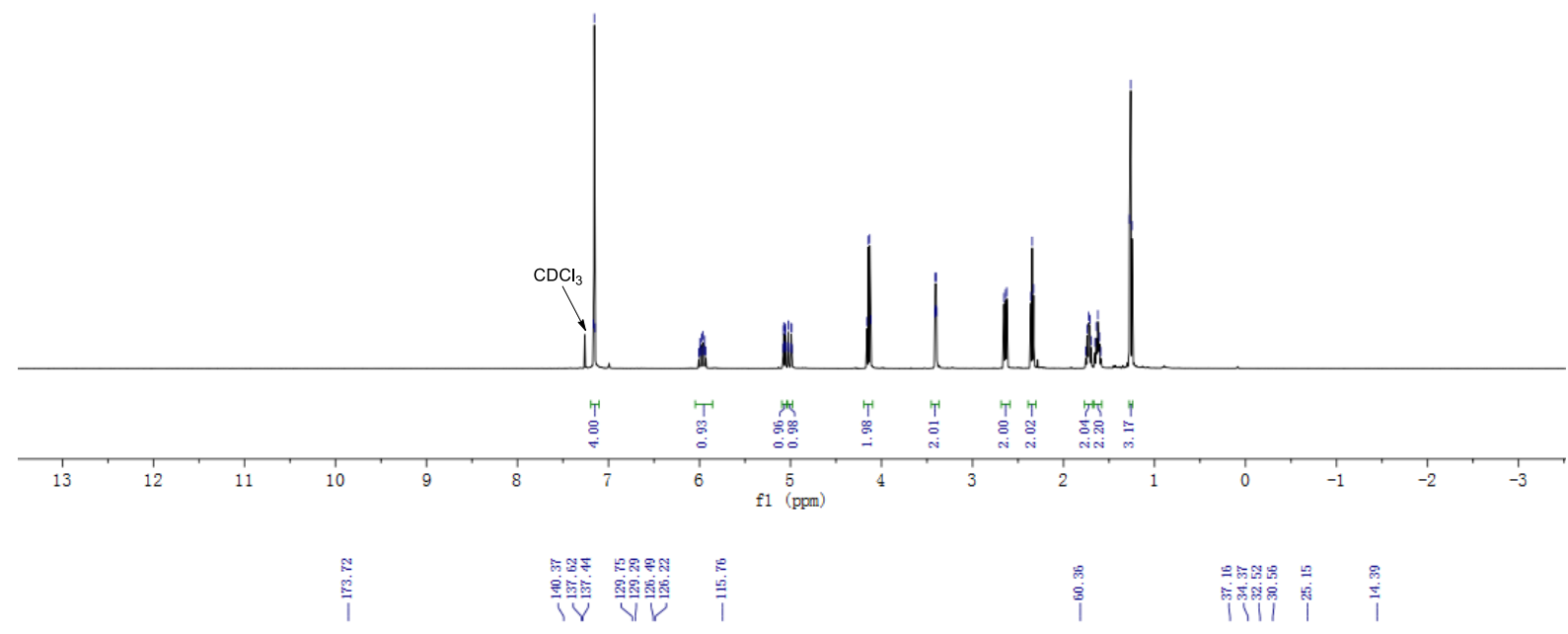<smiles>C=CCc1ccccc1CCCCC(=O)OCC</smiles>

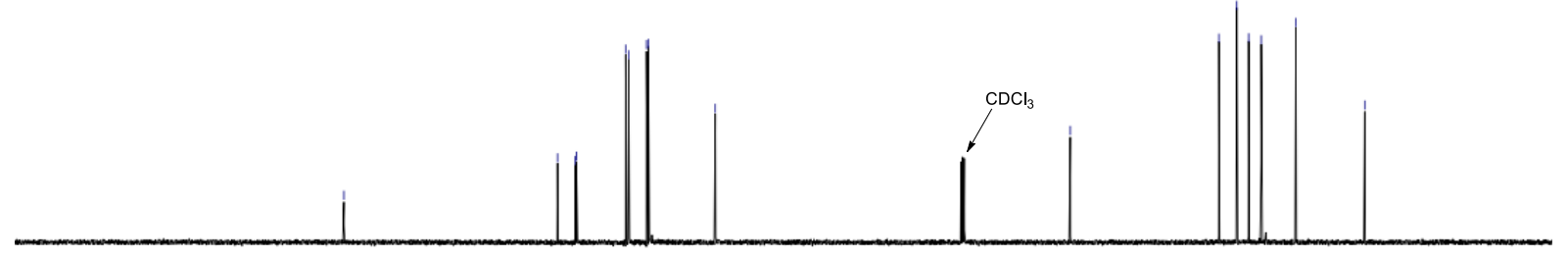

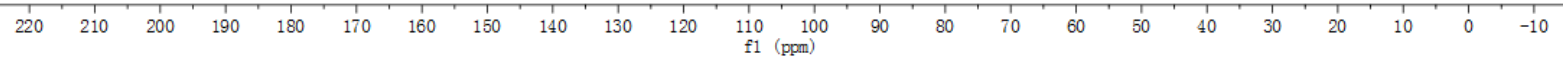


${ }^{1} \mathrm{H}$ NMR $\left(500 \mathrm{MHz}, \mathrm{CDCl}_{3}\right),{ }^{13} \mathrm{C} \mathrm{NMR}\left(126 \mathrm{MHz}, \mathrm{CDCl}_{3}\right)$
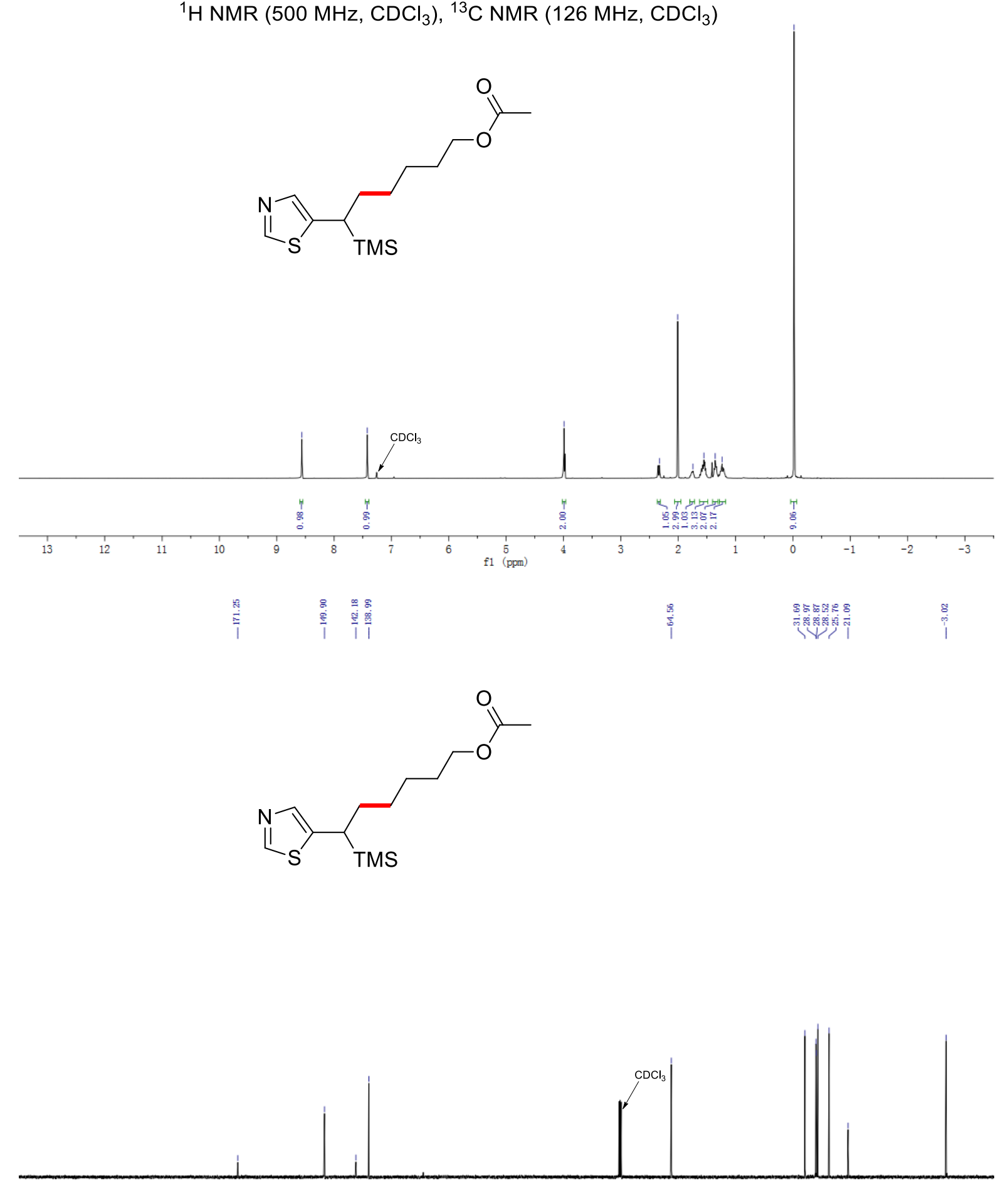

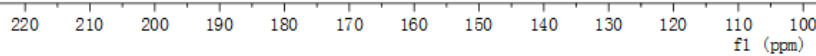




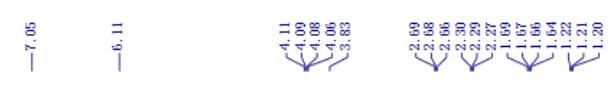

${ }^{1} \mathrm{H}$ NMR $\left(500 \mathrm{MHz}, \mathrm{CDCl}_{3}\right),{ }^{13} \mathrm{C} \mathrm{NMR}\left(126 \mathrm{MHz}, \mathrm{CDCl}_{3}\right)$<smiles>CCOC(=O)CCCCc1ccc(C(=O)OC)o1</smiles>

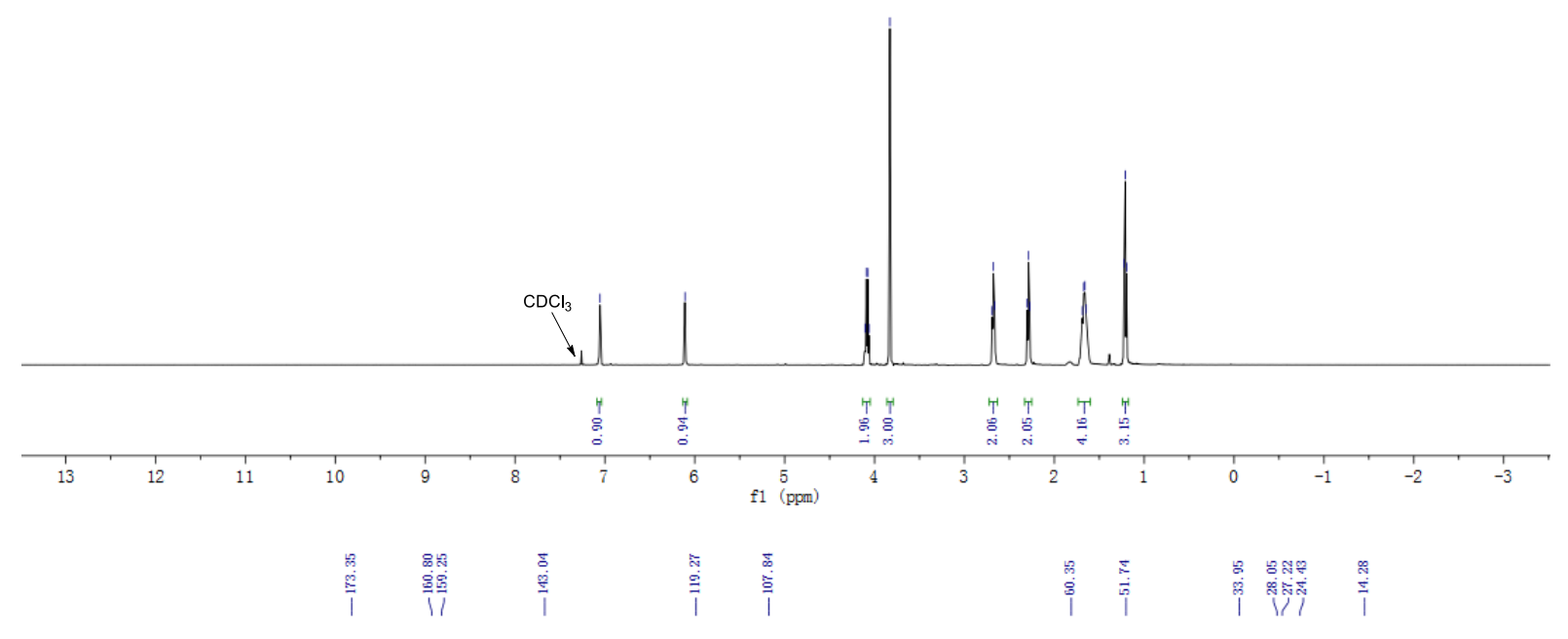<smiles>CCOC(=O)CCCCc1ccc(C(=O)OC)o1</smiles>

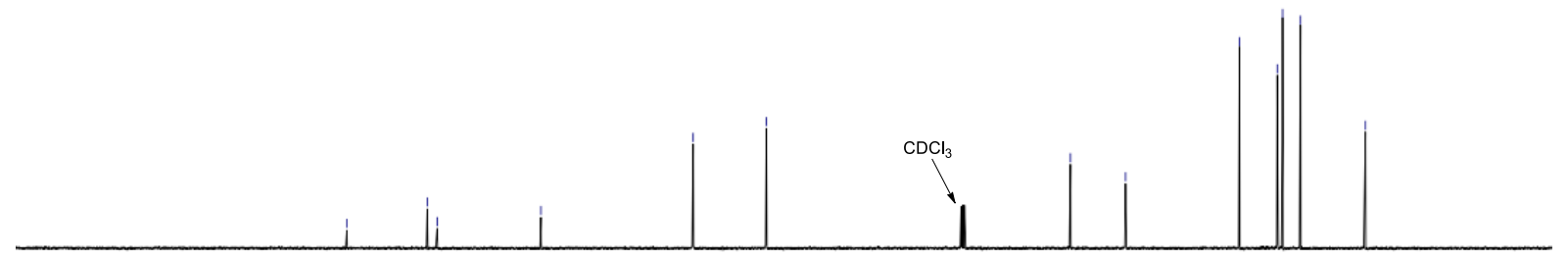

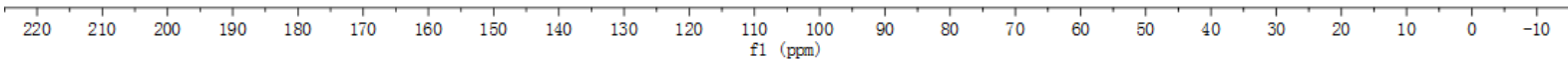


${ }^{1} \mathrm{H}$ NMR $\left(500 \mathrm{MHz}, \mathrm{CDCl}_{3}\right),{ }^{13} \mathrm{C}$ NMR $\left(126 \mathrm{MHz}, \mathrm{CDCl}_{3}\right)$
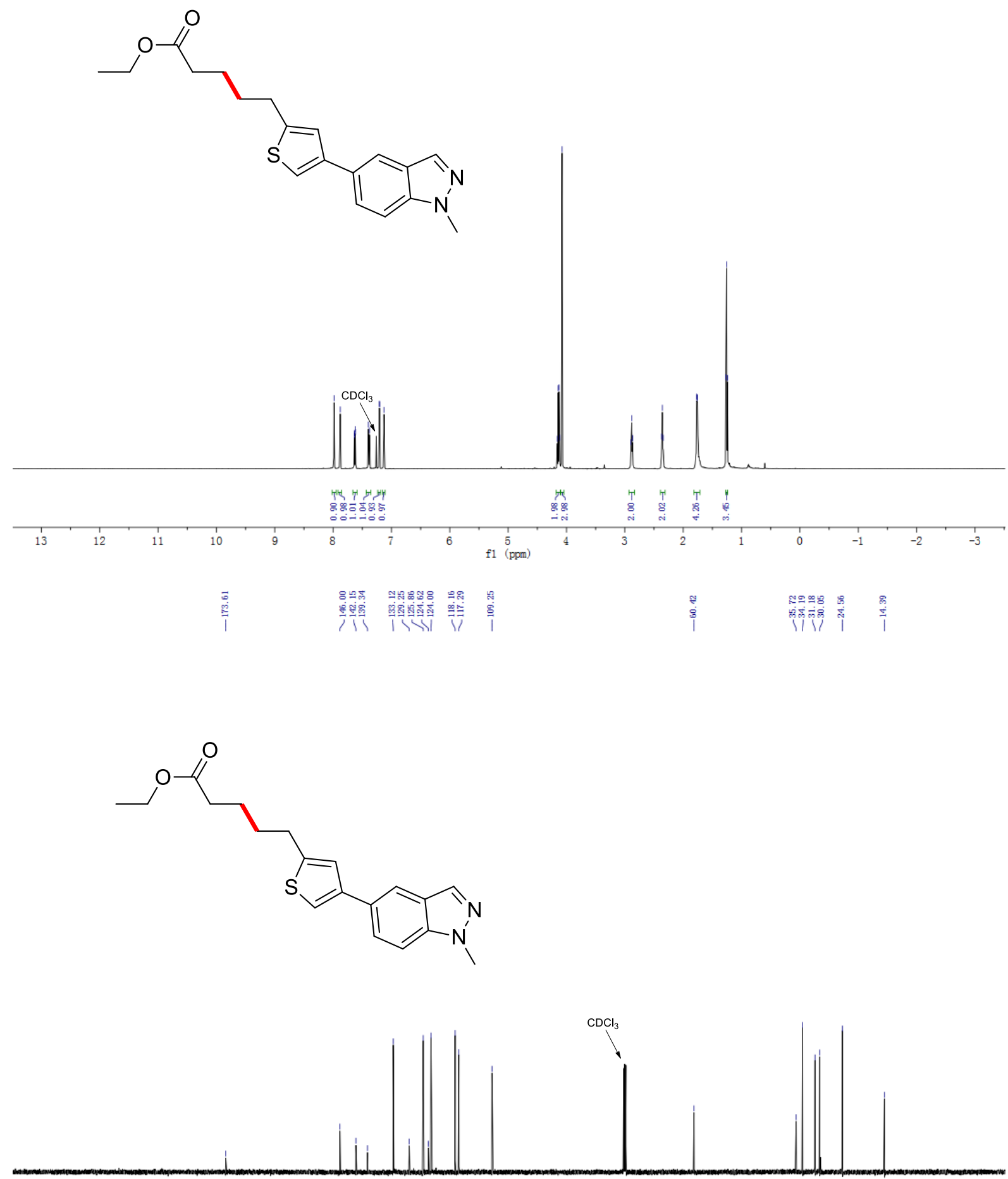

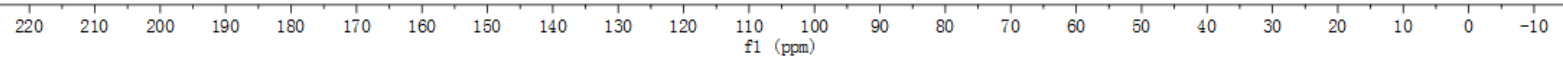




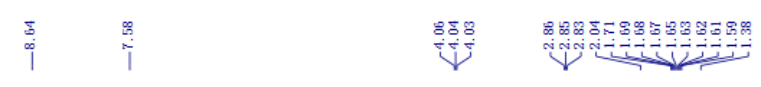

${ }^{1} \mathrm{H}$ NMR $\left(500 \mathrm{MHz}, \mathrm{CDCl}_{3}\right),{ }^{13} \mathrm{C} \mathrm{NMR}\left(126 \mathrm{MHz}, \mathrm{CDCl}_{3}\right)$
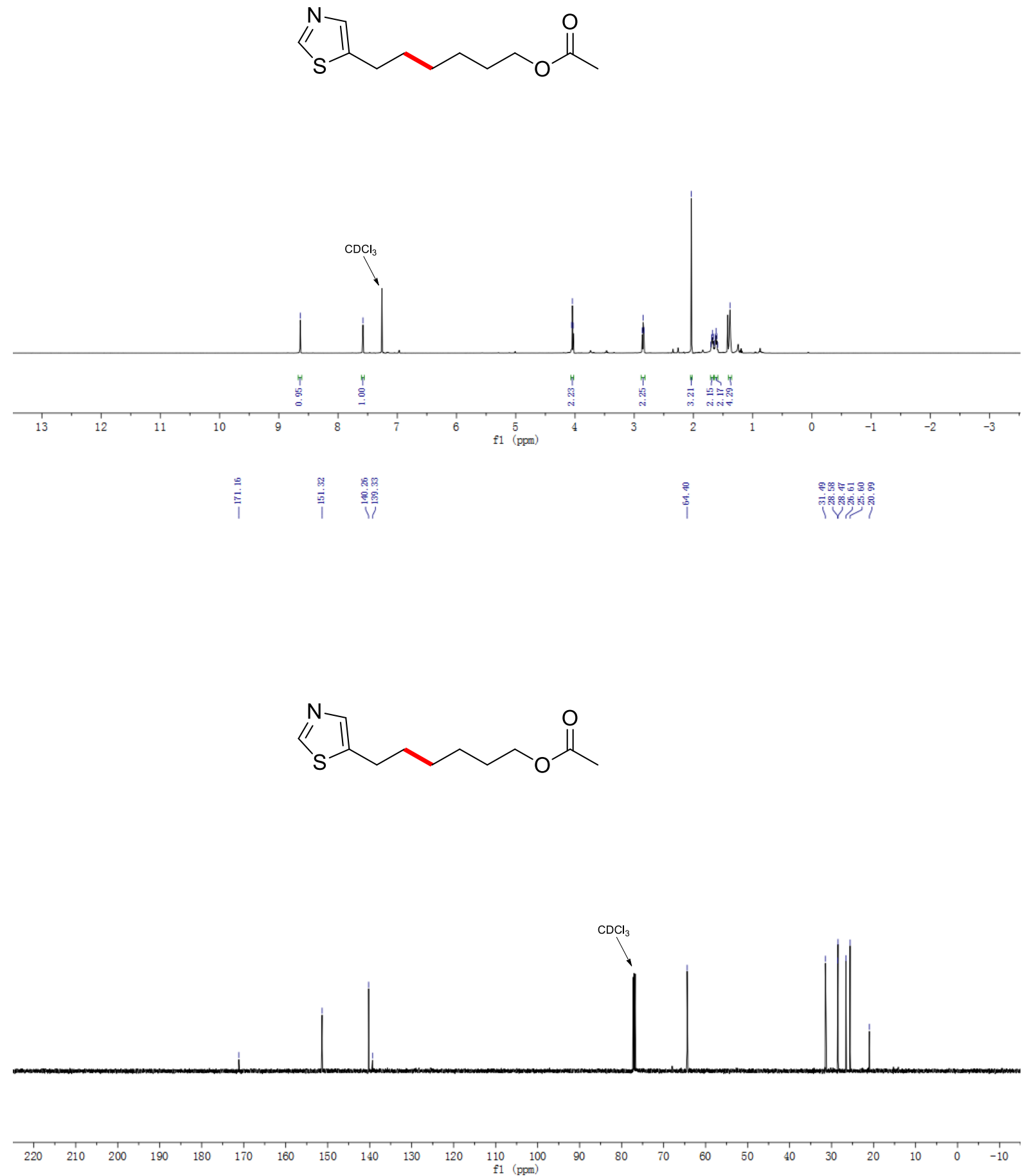


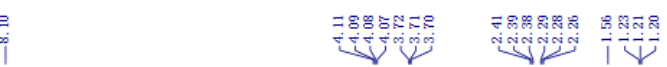

${ }^{1} \mathrm{H}$ NMR $\left(500 \mathrm{MHz}, \mathrm{CDCl}_{3}\right),{ }^{13} \mathrm{C} \mathrm{NMR}\left(126 \mathrm{MHz}, \mathrm{CDCl}_{3}\right)$
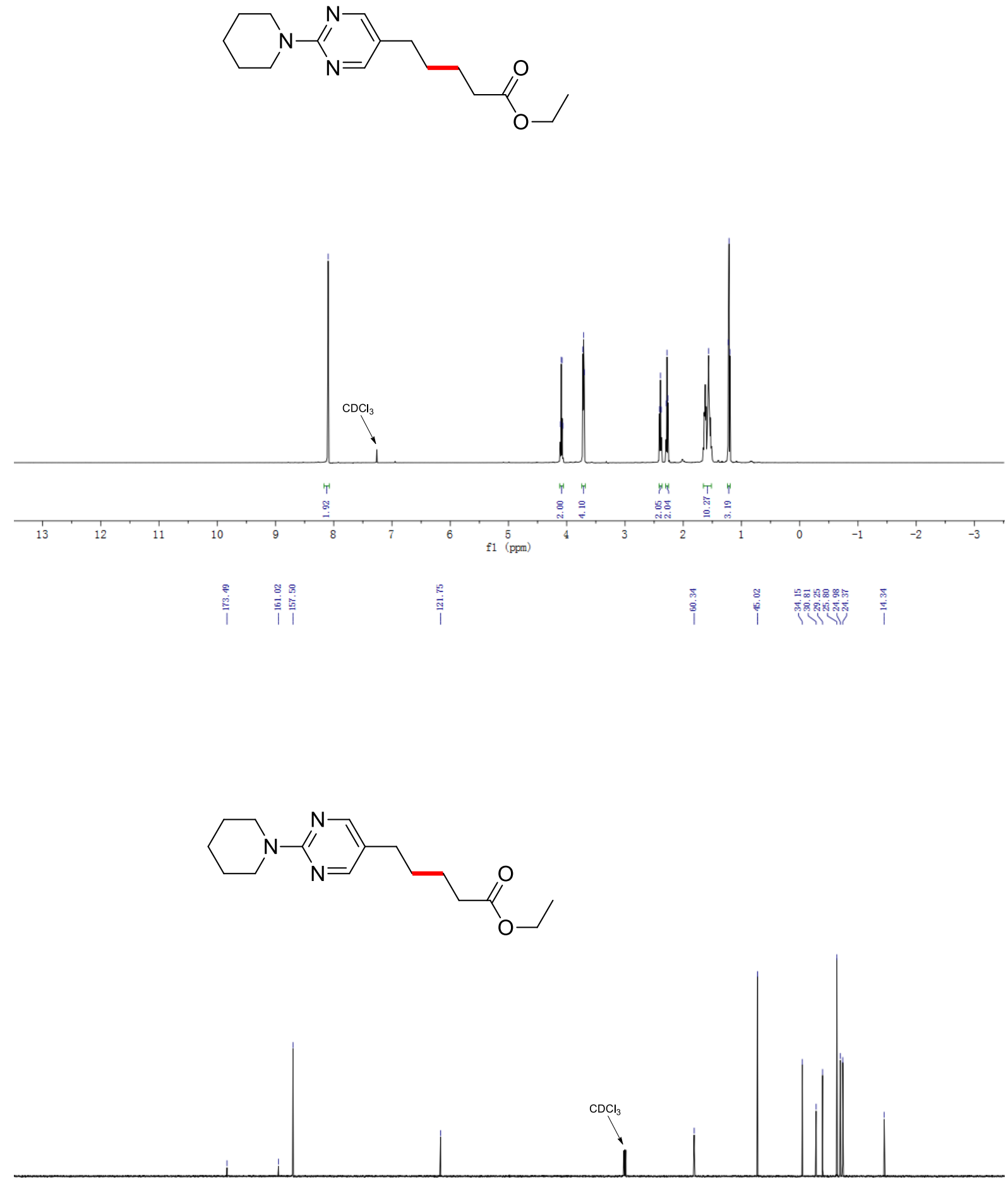

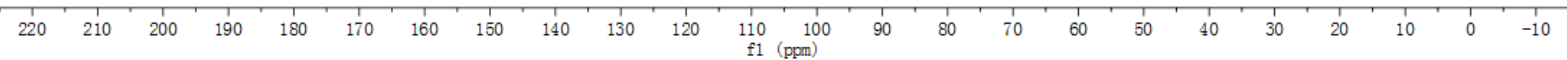




\section{몽ำ}

${ }^{1} \mathrm{H}$ NMR $\left(500 \mathrm{MHz}, \mathrm{CDCl}_{3}\right),{ }^{13} \mathrm{C} \mathrm{NMR}\left(126 \mathrm{MHz}, \mathrm{CDCl}_{3}\right)$
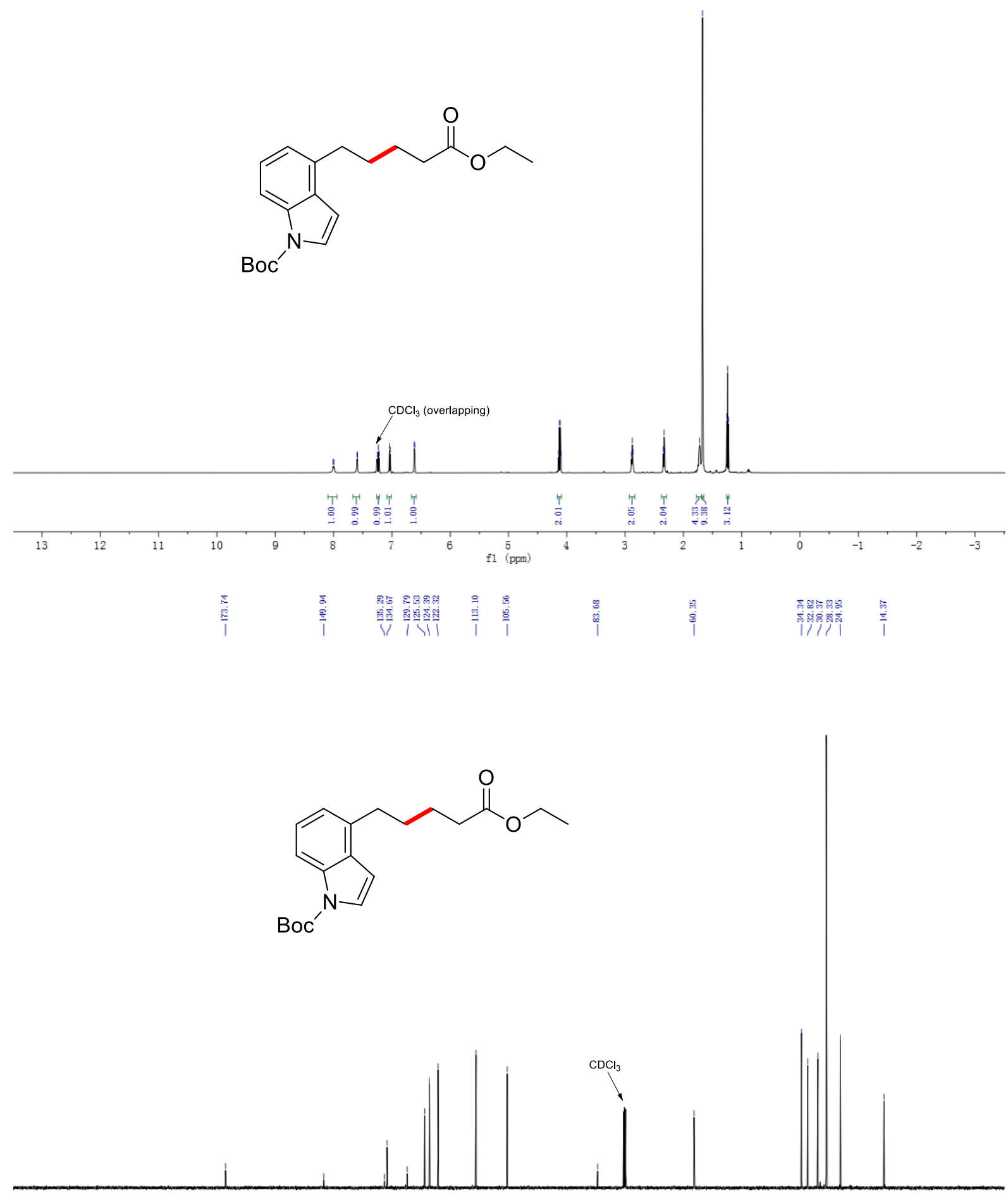

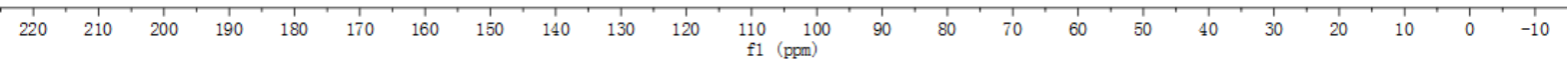


${ }^{1} \mathrm{H}$ NMR $\left(500 \mathrm{MHz}, \mathrm{CDCl}_{3}\right),{ }^{13} \mathrm{C} \mathrm{NMR}\left(126 \mathrm{MHz} \mathrm{CDCl}_{3}\right)$

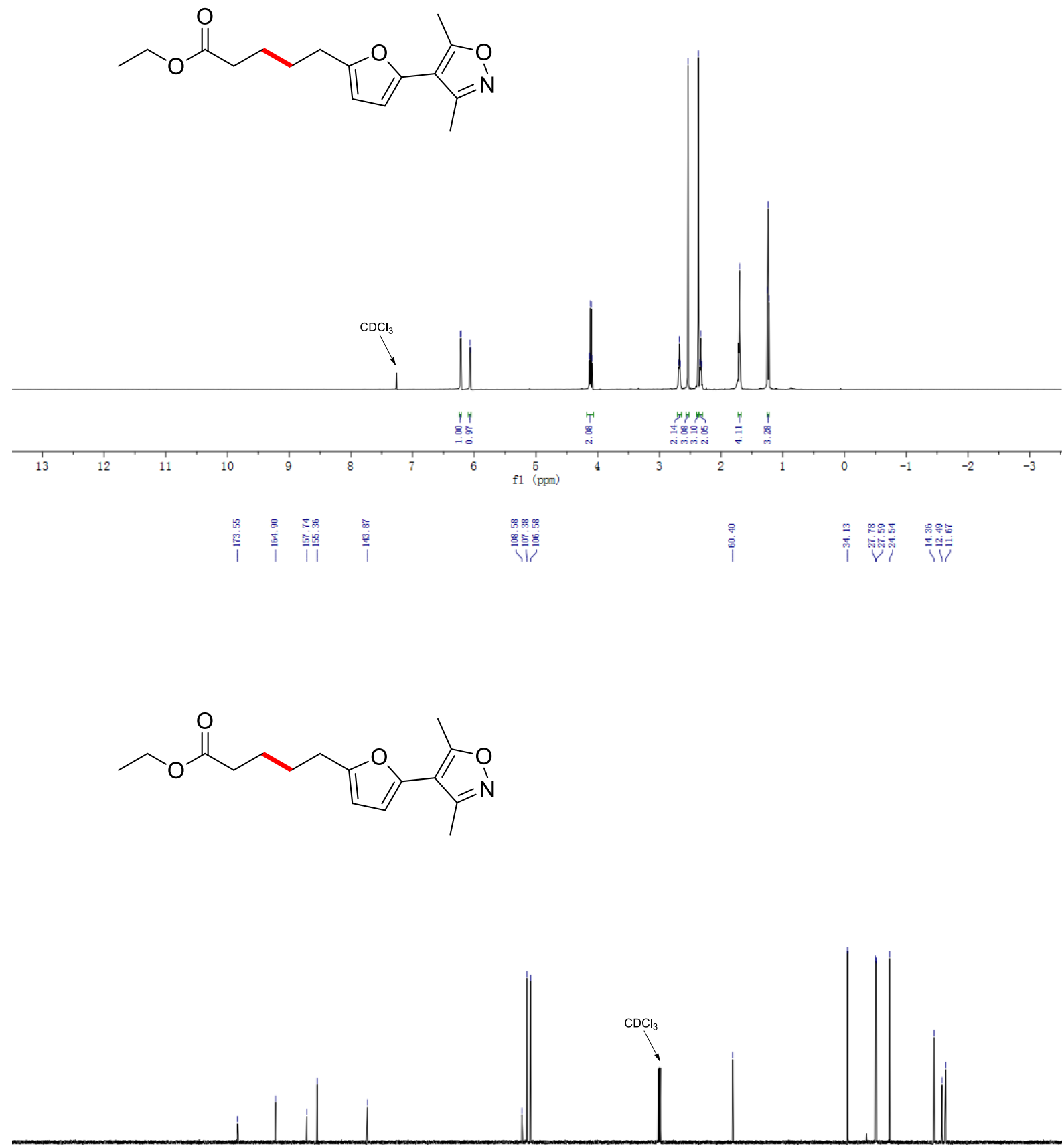

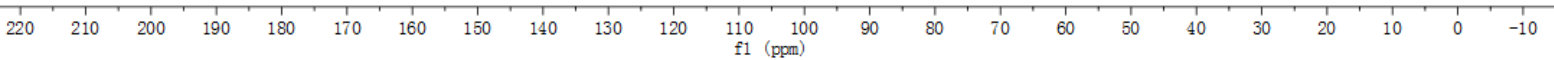




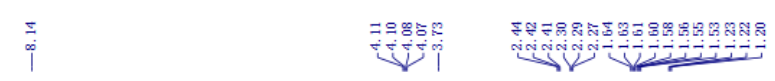

${ }^{1} \mathrm{H}$ NMR $\left(500 \mathrm{MHz}, \mathrm{CDCl}_{3}\right),{ }^{13} \mathrm{C} \mathrm{NMR}\left(126 \mathrm{MHz}, \mathrm{CDCl}_{3}\right)$
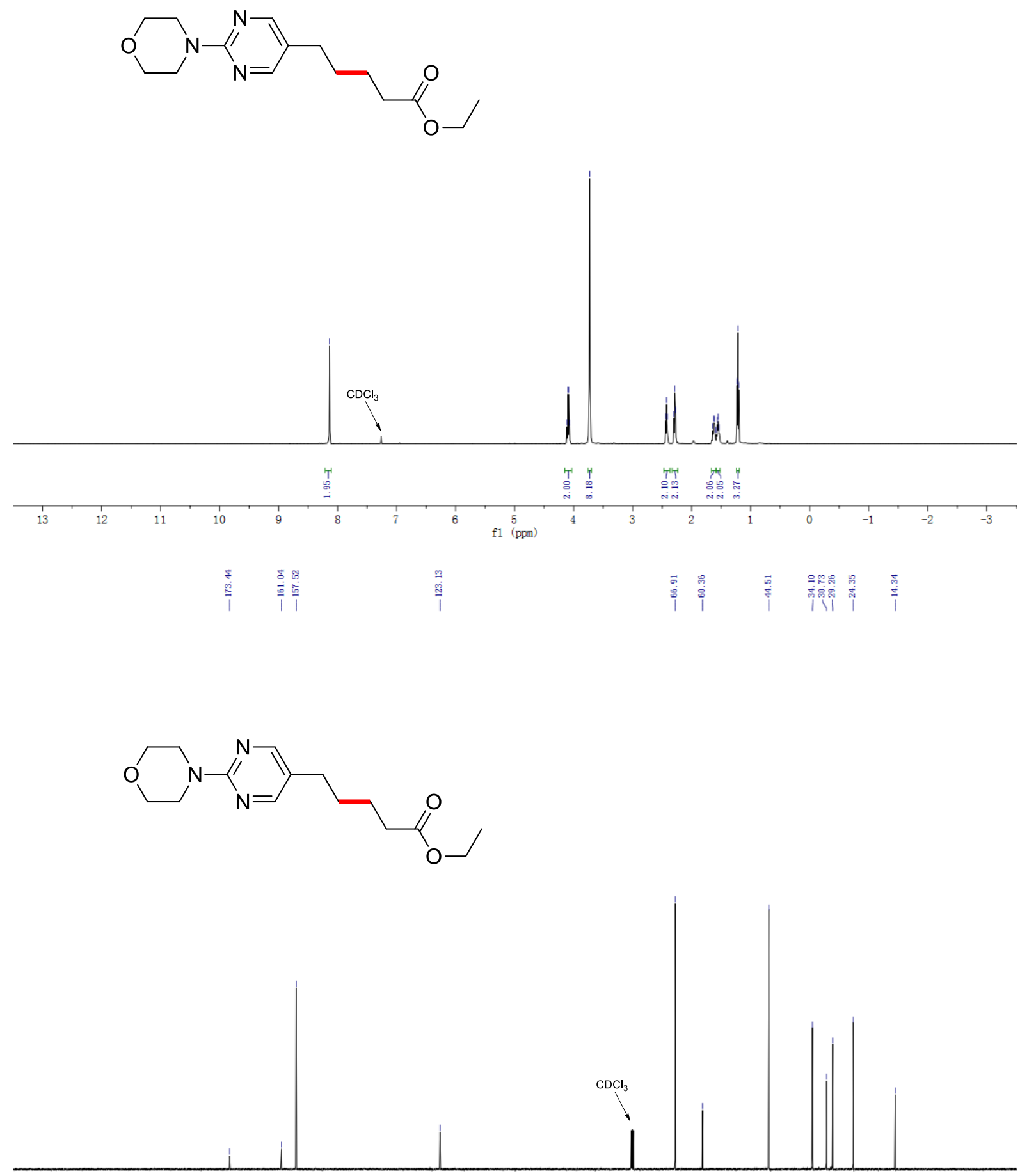

$\begin{array}{llllllllllllllllllllllllll}1 & 1 & 210 & 200 & 190 & 180 & 170 & 160 & 150 & 140 & 130 & 120 & 110 & 100 & 90 & 80 & 70 & 60 & 50 & 40 & 30 & 20 & 10 & 0 & -10\end{array}$ 
${ }^{1} \mathrm{H}$ NMR $\left(500 \mathrm{MHz}, \mathrm{CDCl}_{3}\right),{ }^{13} \mathrm{C} \mathrm{NMR}\left(126 \mathrm{MHz}, \mathrm{CDCl}_{3}\right)$
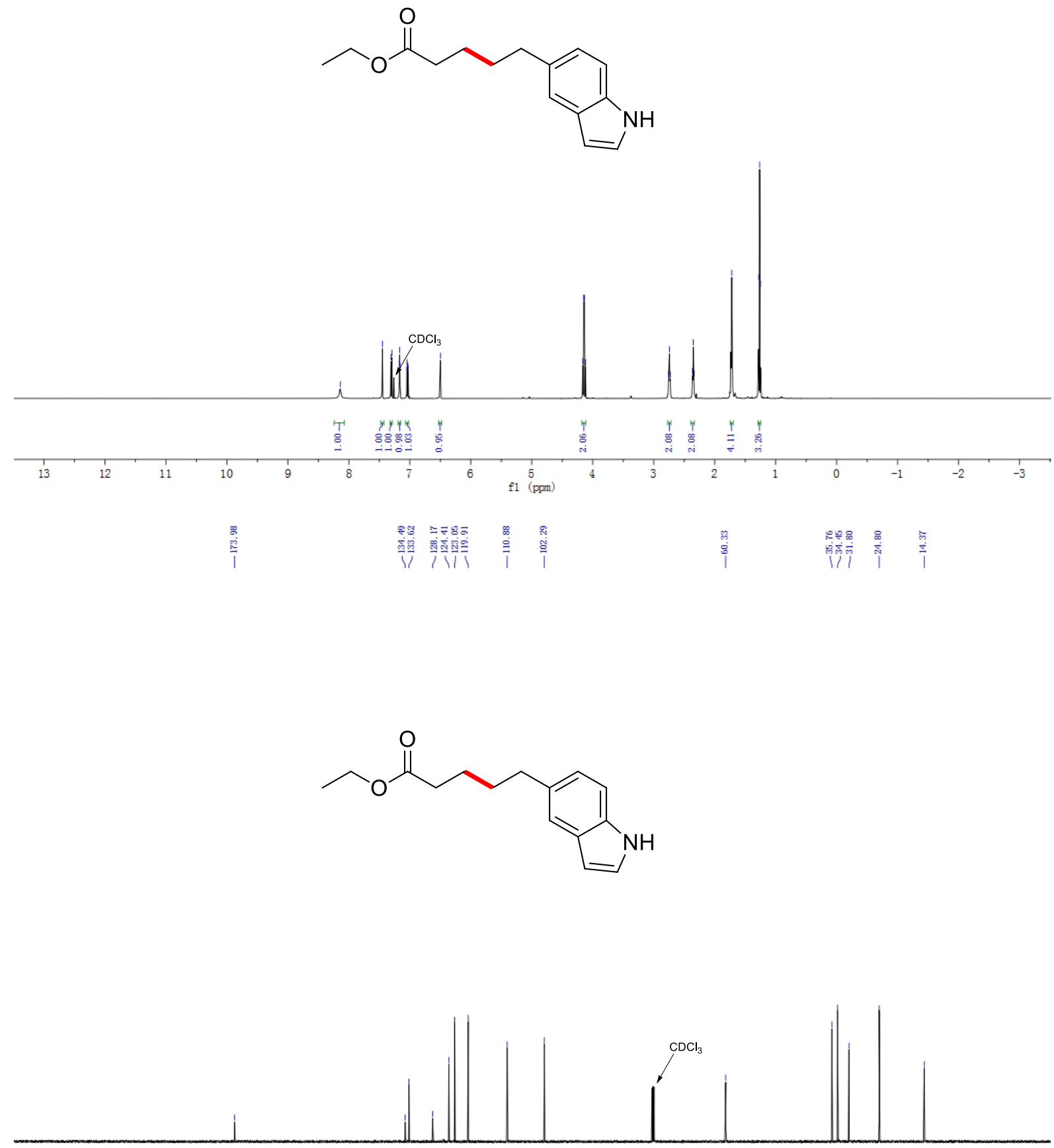

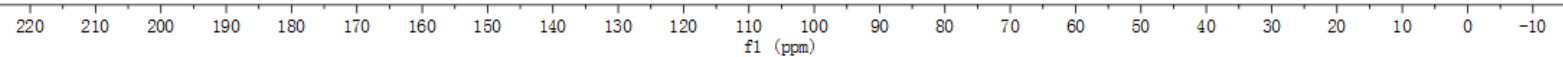


${ }^{1} \mathrm{H}$ NMR $\left(400 \mathrm{MHz}, \mathrm{CDCl}_{3}\right),{ }^{13} \mathrm{C} \mathrm{NMR}\left(126 \mathrm{MHz} \mathrm{CDCl}_{3}\right)$<smiles>Cc1noc(C)c1NC(=O)c1ccc(CCCCC(=O)N2CCN(c3cccc(Br)c3)CC2)cc1</smiles>

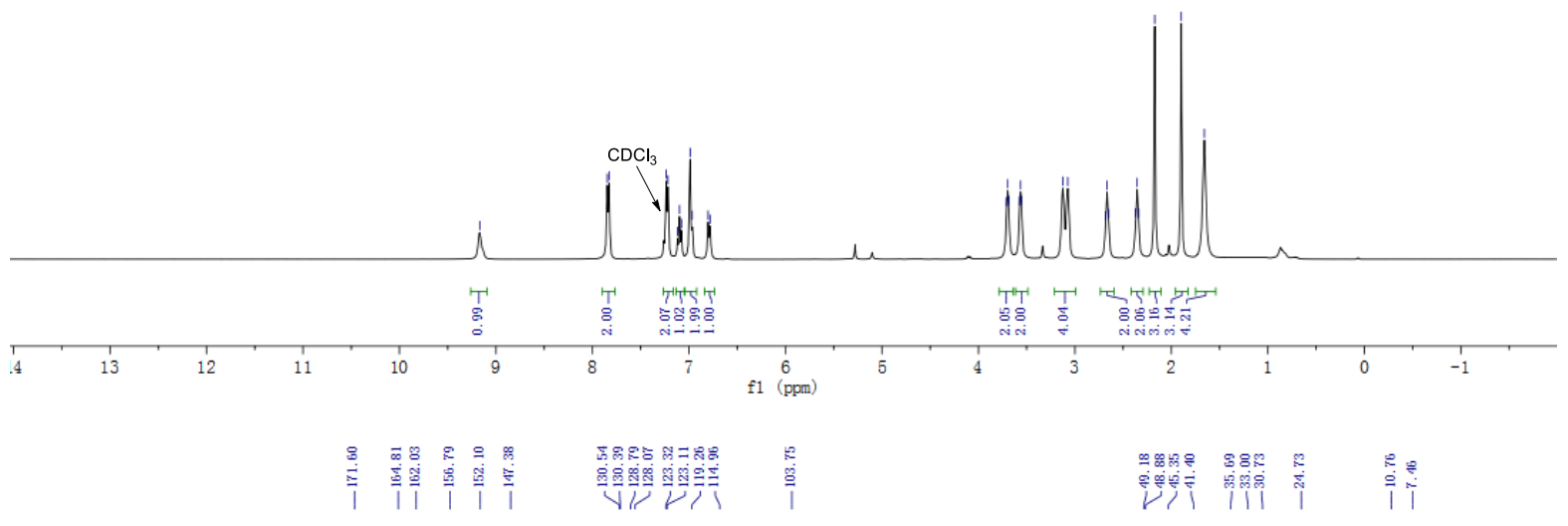<smiles>Cc1noc(C)c1NC(=O)c1ccc(CCCCC(=O)N2CCN(c3cccc(Br)c3)CC2)cc1</smiles>

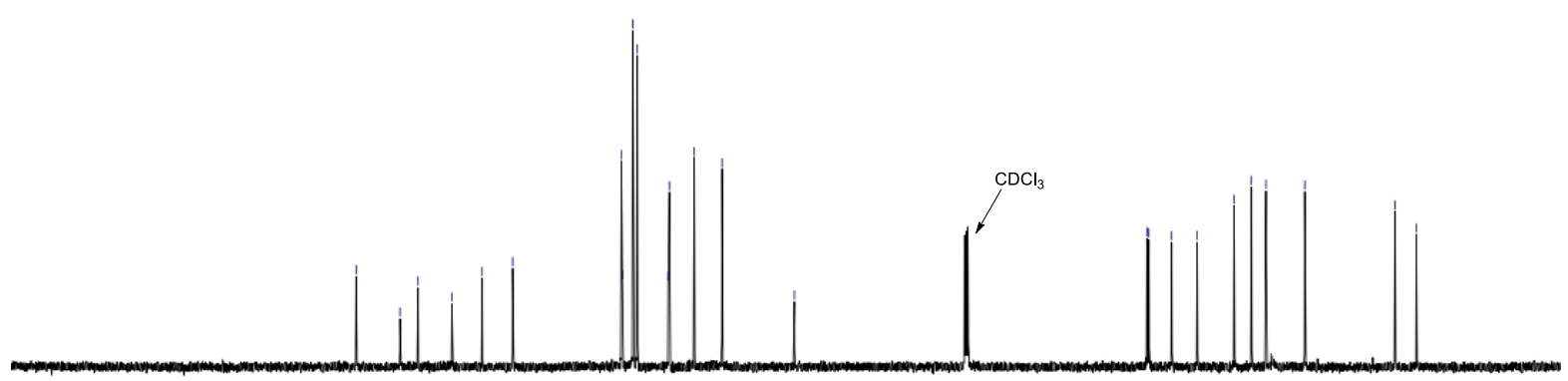

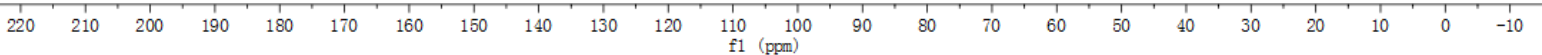


${ }^{1} \mathrm{H}$ NMR $\left(500 \mathrm{MHz}, \mathrm{CDCl}_{3}\right),{ }^{13} \mathrm{C} \mathrm{NMR}\left(126 \mathrm{MHz}, \mathrm{CDCl}_{3}\right)$
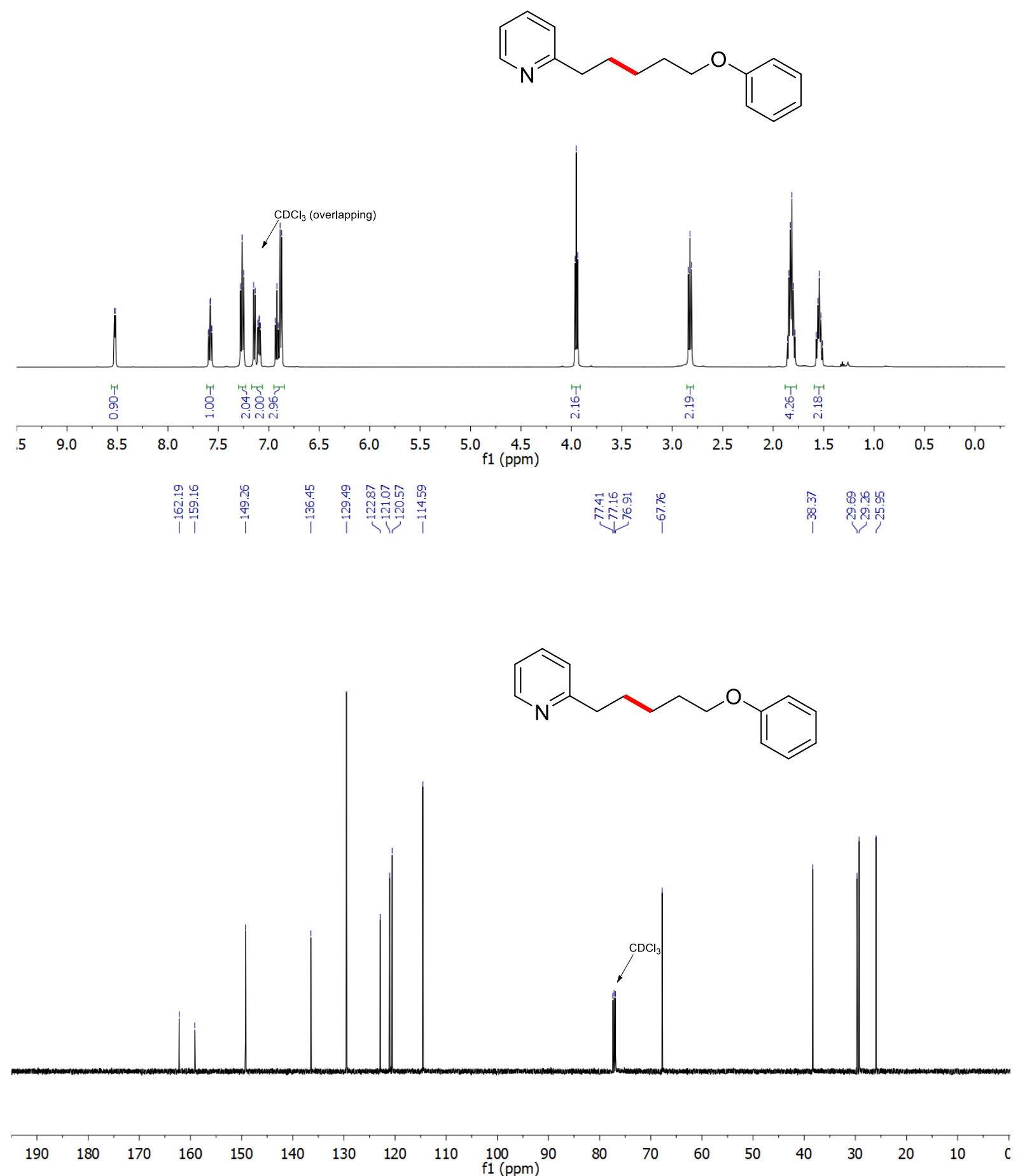
${ }^{1} \mathrm{H} \mathrm{NMR}\left(500 \mathrm{MHz}, \mathrm{CDCl}_{3}\right),{ }^{13} \mathrm{C} \mathrm{NMR}\left(126 \mathrm{MHz}, \mathrm{CDCl}_{3}\right)$
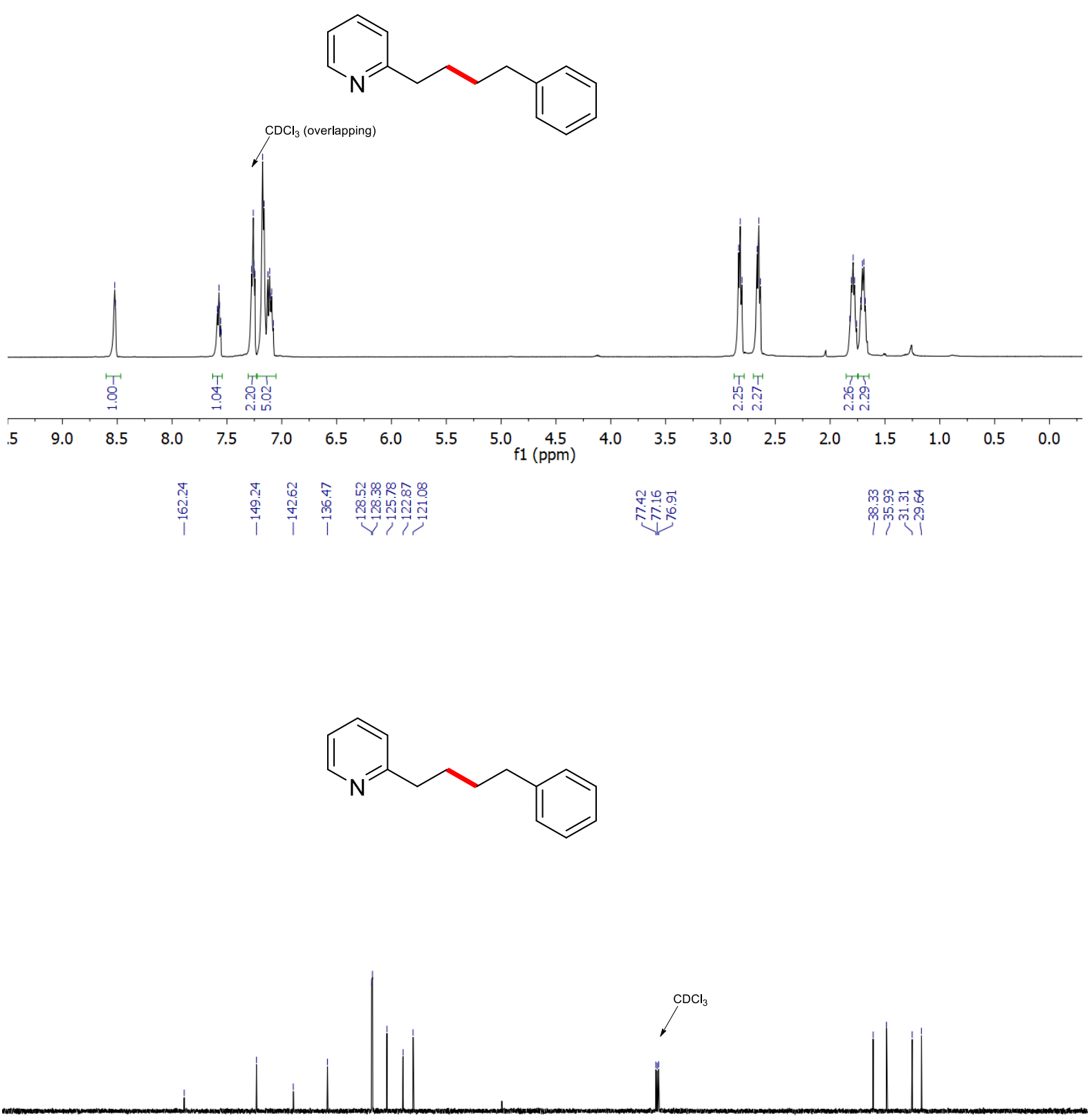


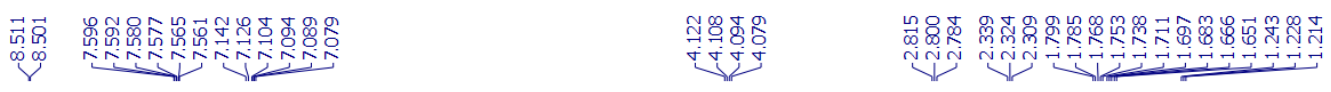

${ }^{1} \mathrm{H}$ NMR $\left(500 \mathrm{MHz}, \mathrm{CDCl}_{3}\right),{ }^{13} \mathrm{C} \mathrm{NMR}\left(126 \mathrm{MHz} \mathrm{CDCl}_{3}\right)$<smiles>CCOC(=O)CCCCc1ccccn1</smiles>

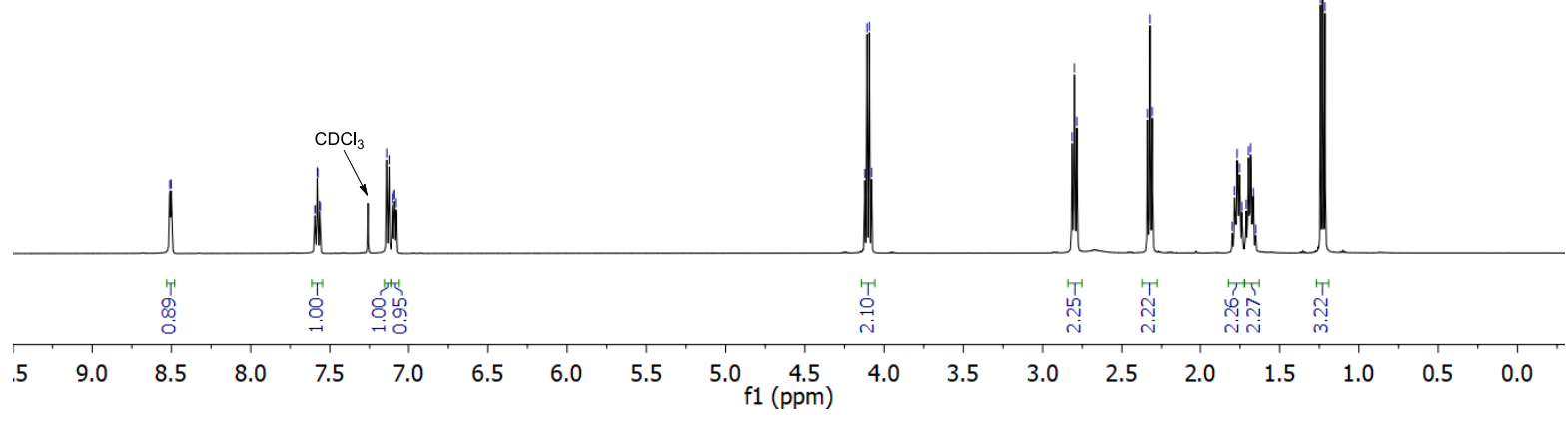

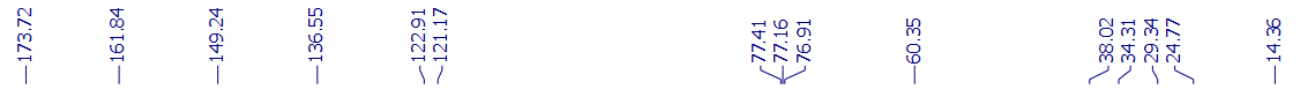<smiles>CCOC(=O)CCCCc1ccccn1</smiles>

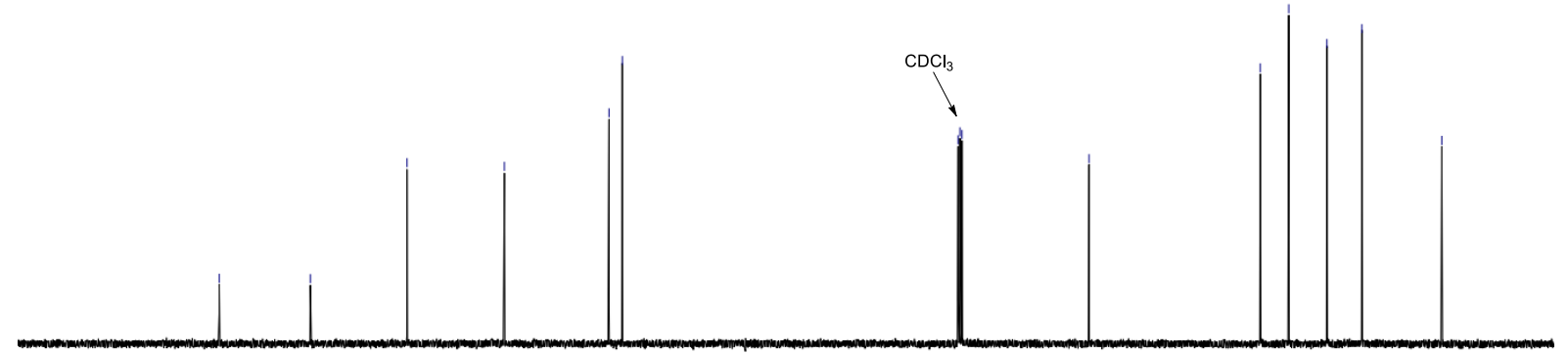

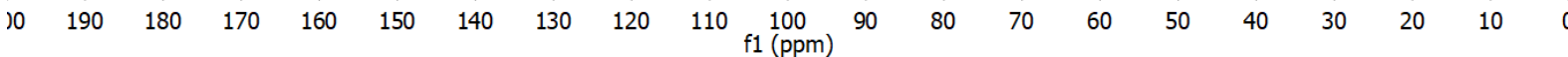


${ }^{1} \mathrm{H} \mathrm{NMR}\left(500 \mathrm{MHz}, \mathrm{CDCl}_{3}\right),{ }^{13} \mathrm{C} \mathrm{NMR}\left(126 \mathrm{MHz}, \mathrm{CDCl}_{3}\right)$
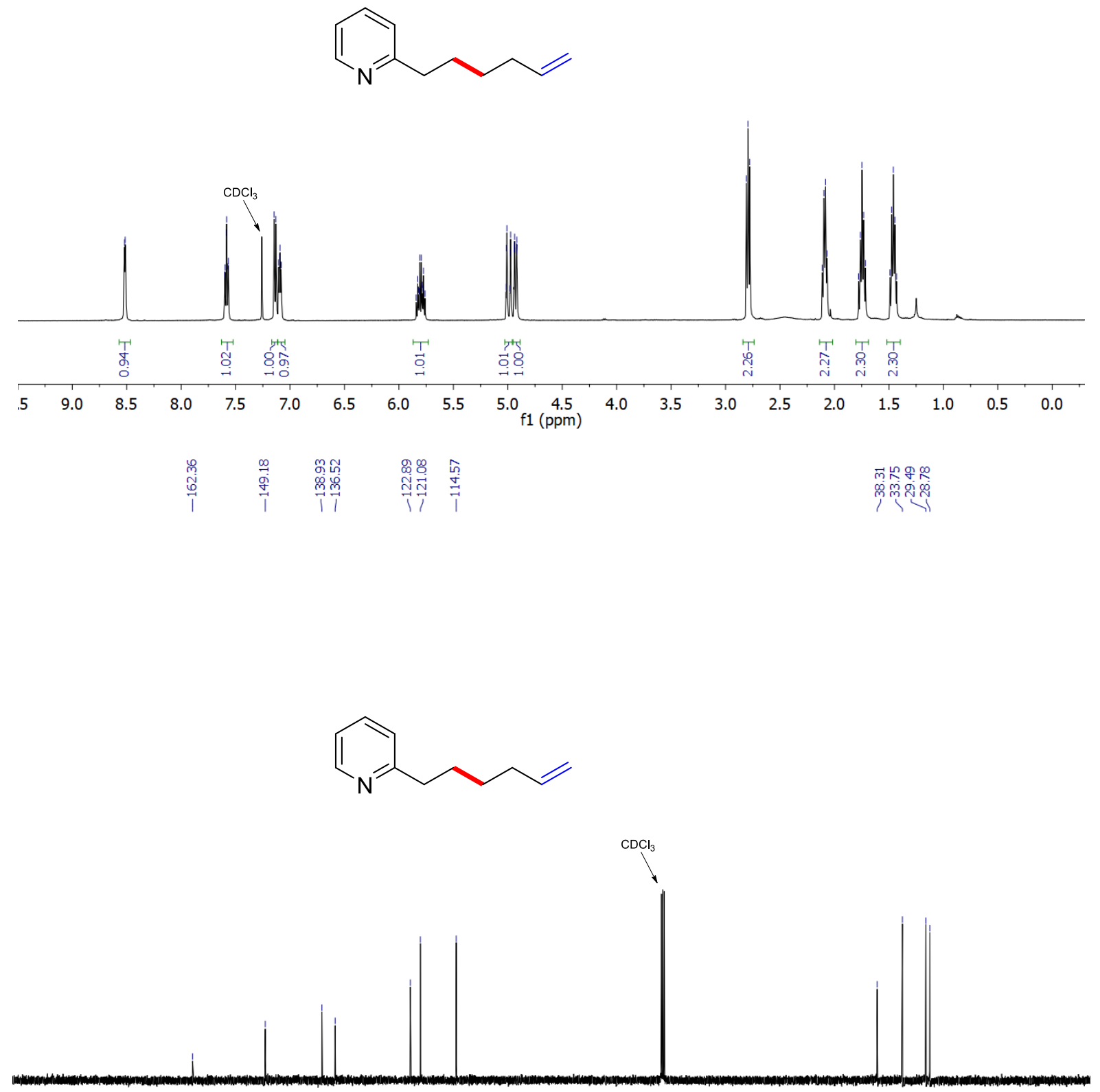


$$
\text { i }
$$

${ }^{1} \mathrm{H} \mathrm{NMR}\left(500 \mathrm{MHz}, \mathrm{CDCl}_{3}\right),{ }^{13} \mathrm{C} \mathrm{NMR}\left(126 \mathrm{MHz}^{\mathrm{CDCl}} 3\right)$<smiles>O=C(CCCCc1ccccn1)OCC1c2ccccc2-c2ccccc21</smiles>

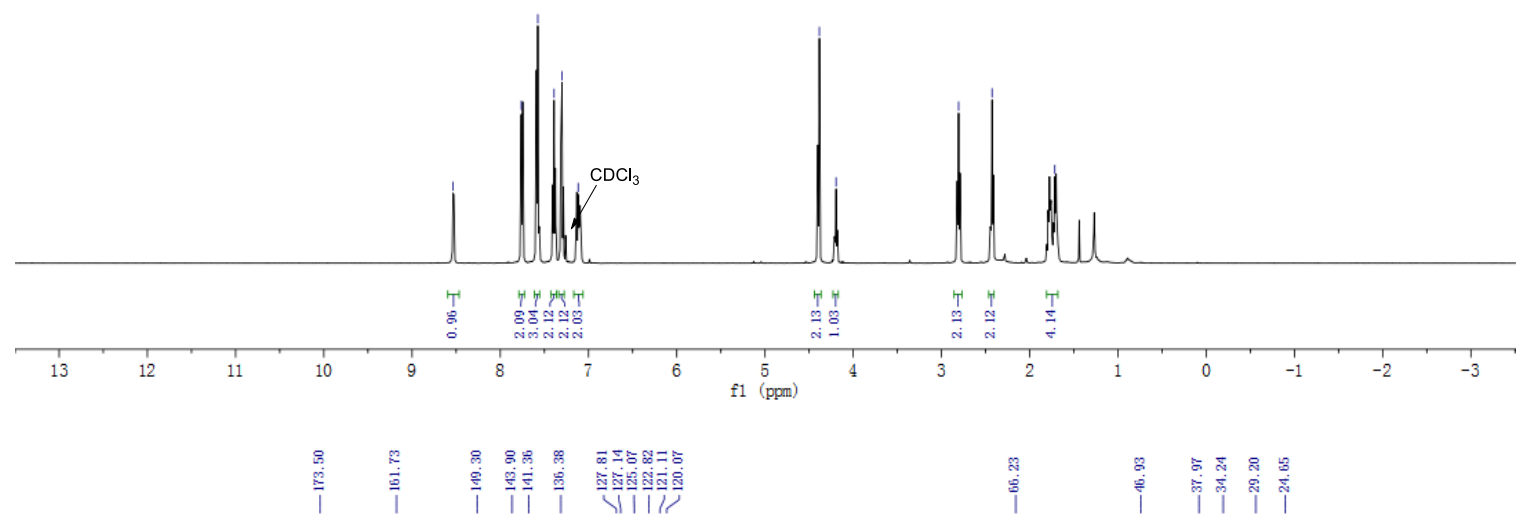<smiles>O=C(CCCCc1ccccn1)OCC1c2ccccc2-c2ccccc21</smiles>

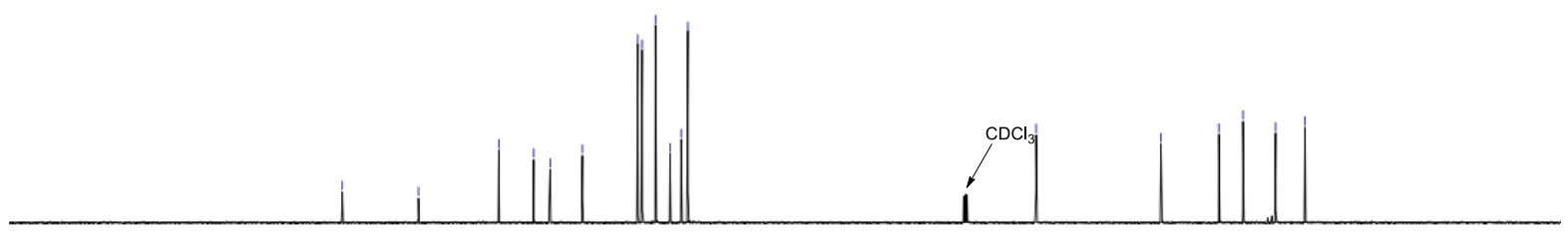

$\begin{array}{lllllllllllll}220 & 210 & 200 & 190 & 180 & 170 & 160 & 150 & 140 & 130 & 120 & 110 & 100 \\ \text { f1 (pm) }\end{array}$ 


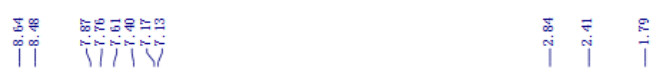

${ }^{1} \mathrm{H} \mathrm{NMR}\left(500 \mathrm{MHz}, \mathrm{CDCl}_{3}\right),{ }^{13} \mathrm{C} \mathrm{NMR}\left(126 \mathrm{MHz}, \mathrm{CDCl}_{3}\right),{ }^{19} \mathrm{~F} \mathrm{NMR}\left(376 \mathrm{MHz}, \mathrm{CDCl}_{3}\right)$<smiles>O=C(CCCCc1ccccn1)Nc1ccc(Cl)c(C(F)(F)F)c1</smiles>

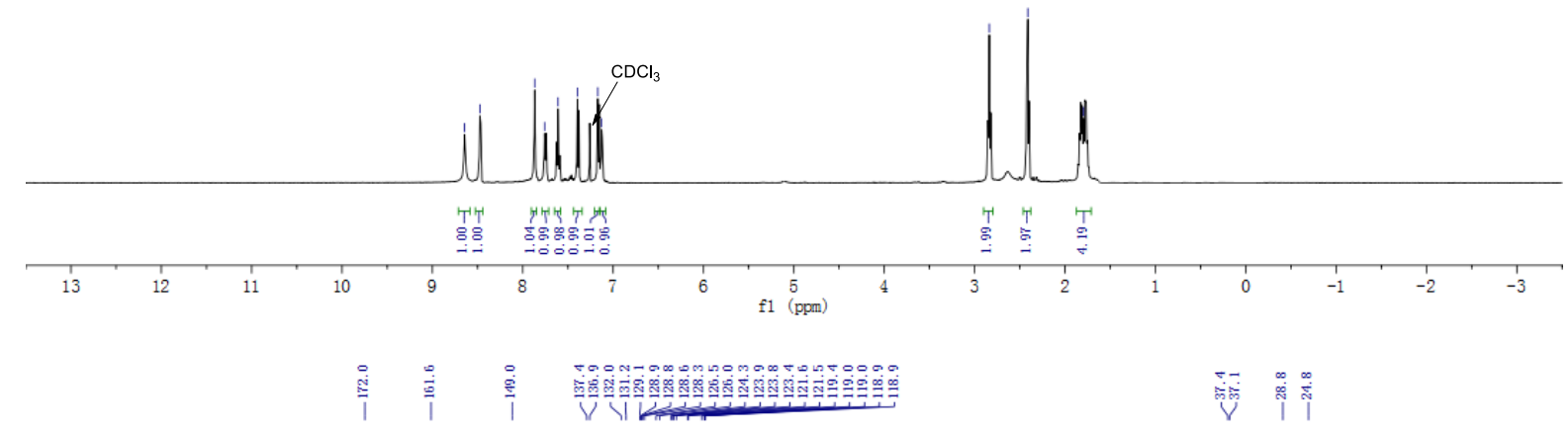<smiles>O=C(CCCCc1ccccn1)Nc1ccc(Cl)c(C(F)(F)F)c1</smiles>

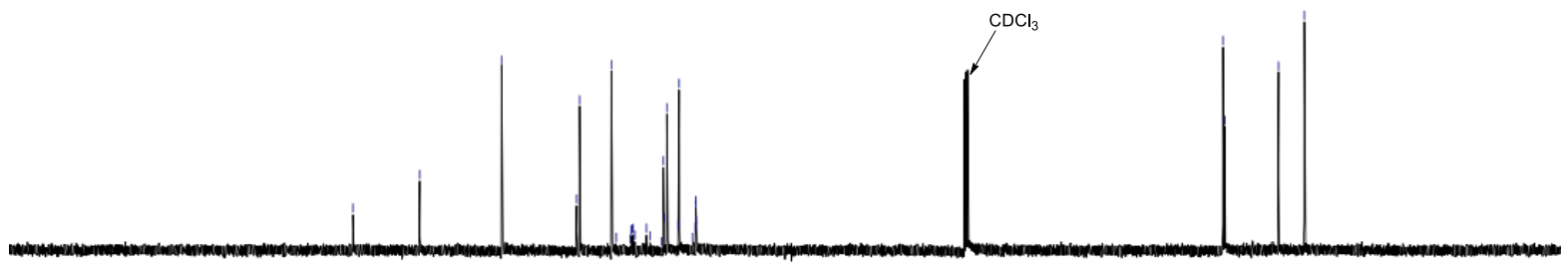

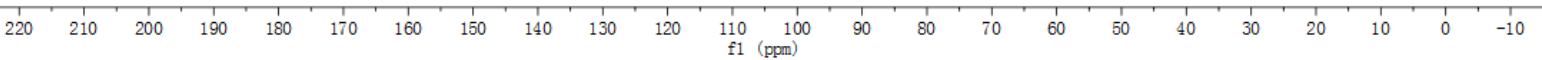




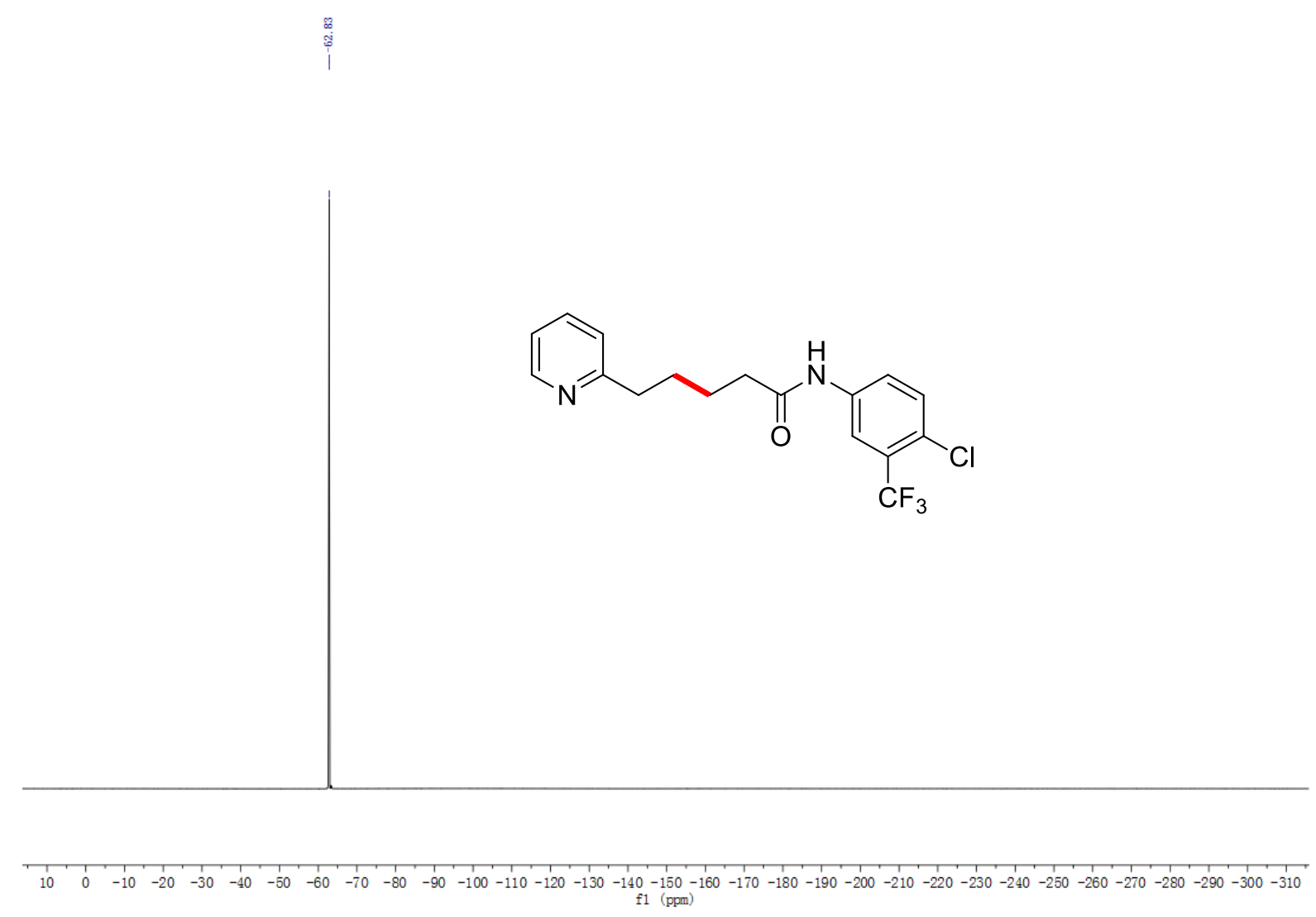




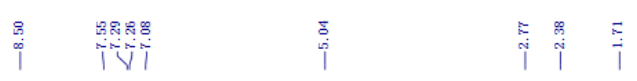

${ }^{1} \mathrm{H}$ NMR $\left(500 \mathrm{MHz}, \mathrm{CDCl}_{3}\right),{ }^{13} \mathrm{C} \mathrm{NMR}\left(126 \mathrm{MHz}, \mathrm{CDCl}_{3}\right)$<smiles>O=C(CCCCc1ccccn1)OCc1ccc(Cl)cc1</smiles>

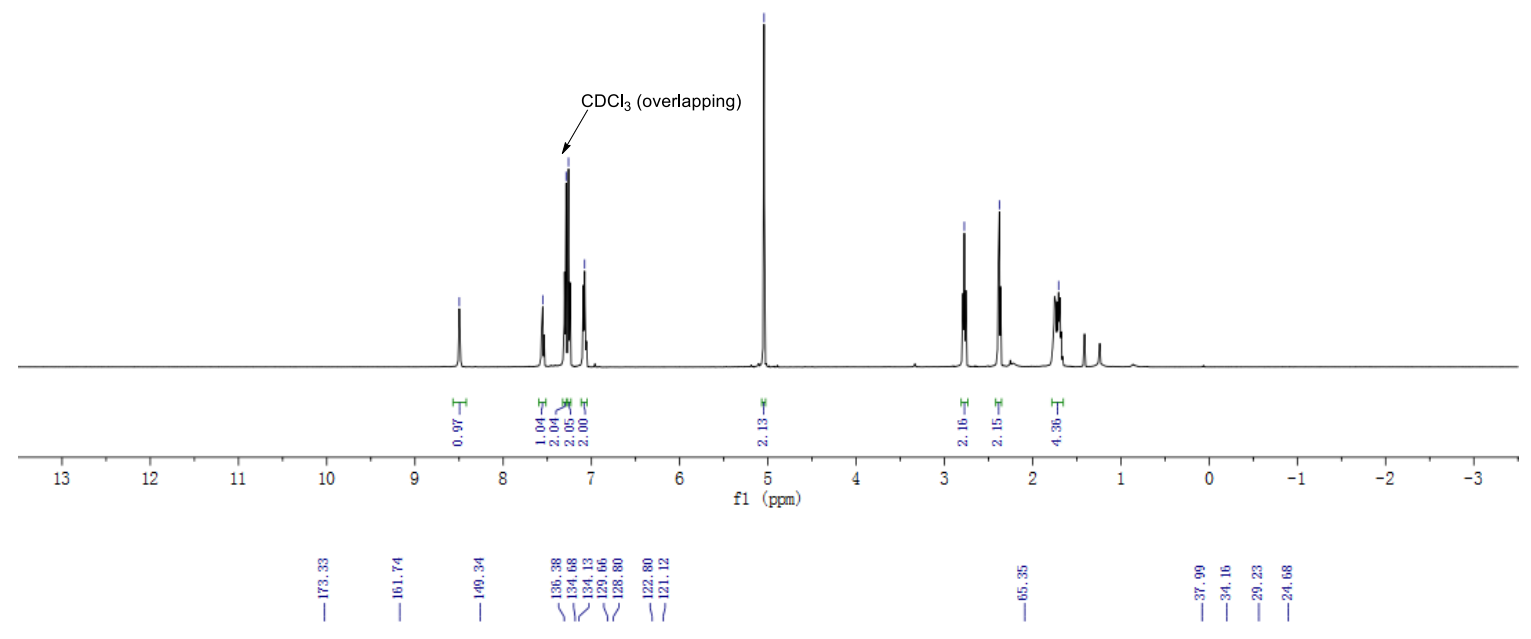<smiles>O=C(CCCCc1ccccn1)OCc1ccc(Cl)cc1</smiles>

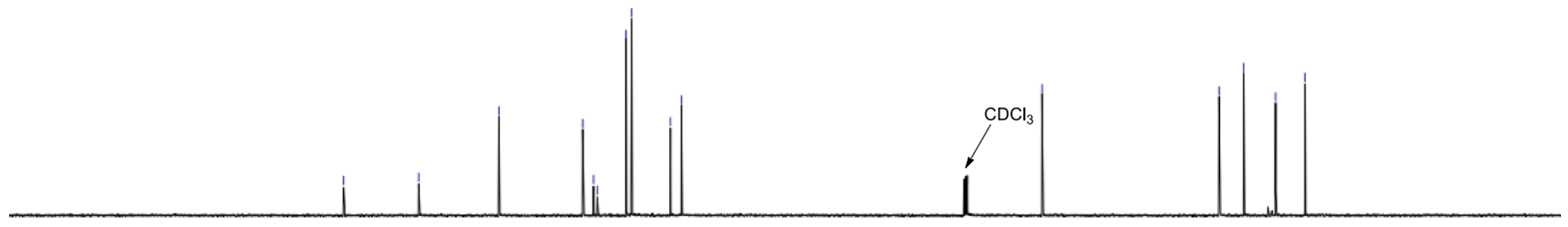

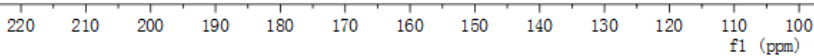




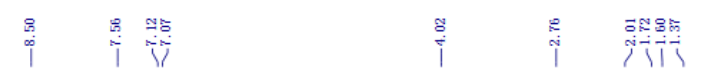

${ }^{1} \mathrm{H}$ NMR $\left(500 \mathrm{MHz}, \mathrm{CDCl}_{3}\right),{ }^{13} \mathrm{C} \mathrm{NMR}\left(126 \mathrm{MHz}, \mathrm{CDCl}_{3}\right)$<smiles>CC(=O)OCCCCCCc1ccccn1</smiles>

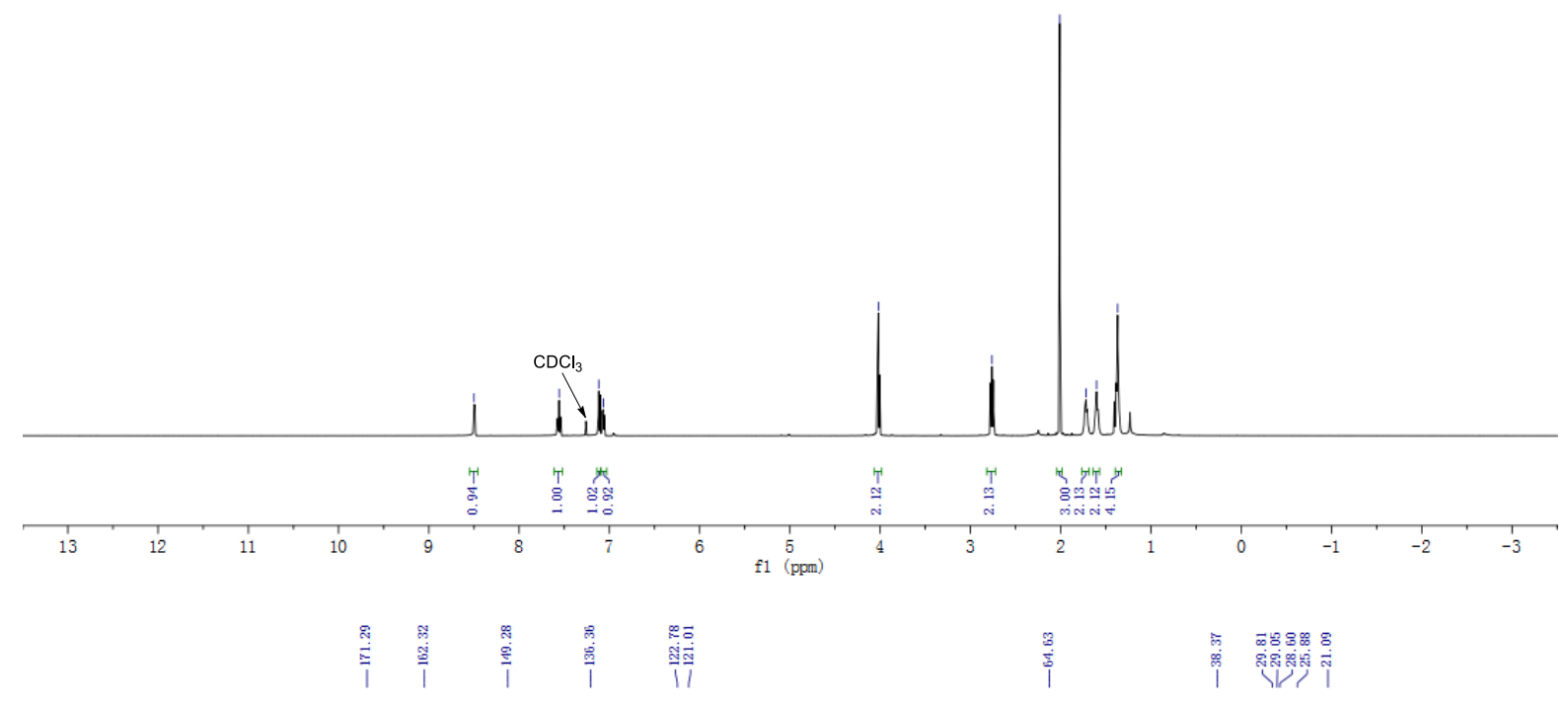<smiles>CC(=O)OCCCCCCc1ccccn1</smiles>

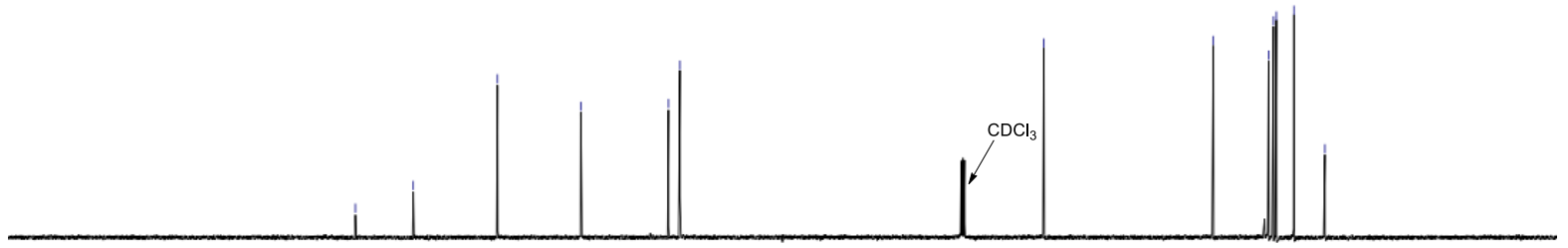

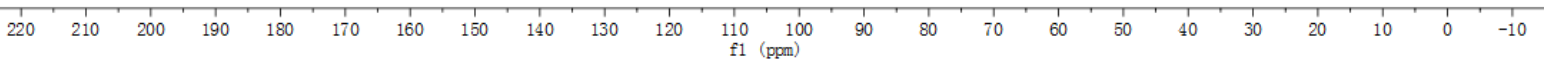


${ }^{1} \mathrm{H} \mathrm{NMR}\left(500 \mathrm{MHz}, \mathrm{CDCl}_{3}\right),{ }^{13} \mathrm{C} \mathrm{NMR}\left(126 \mathrm{MHz}, \mathrm{CDCl}_{3}\right),{ }^{19} \mathrm{~F} \mathrm{NMR}\left(376 \mathrm{MHz}, \mathrm{CDCl}_{3}\right)$<smiles>O=C(CCCCc1ccccn1)OCc1ccc(F)cc1</smiles>

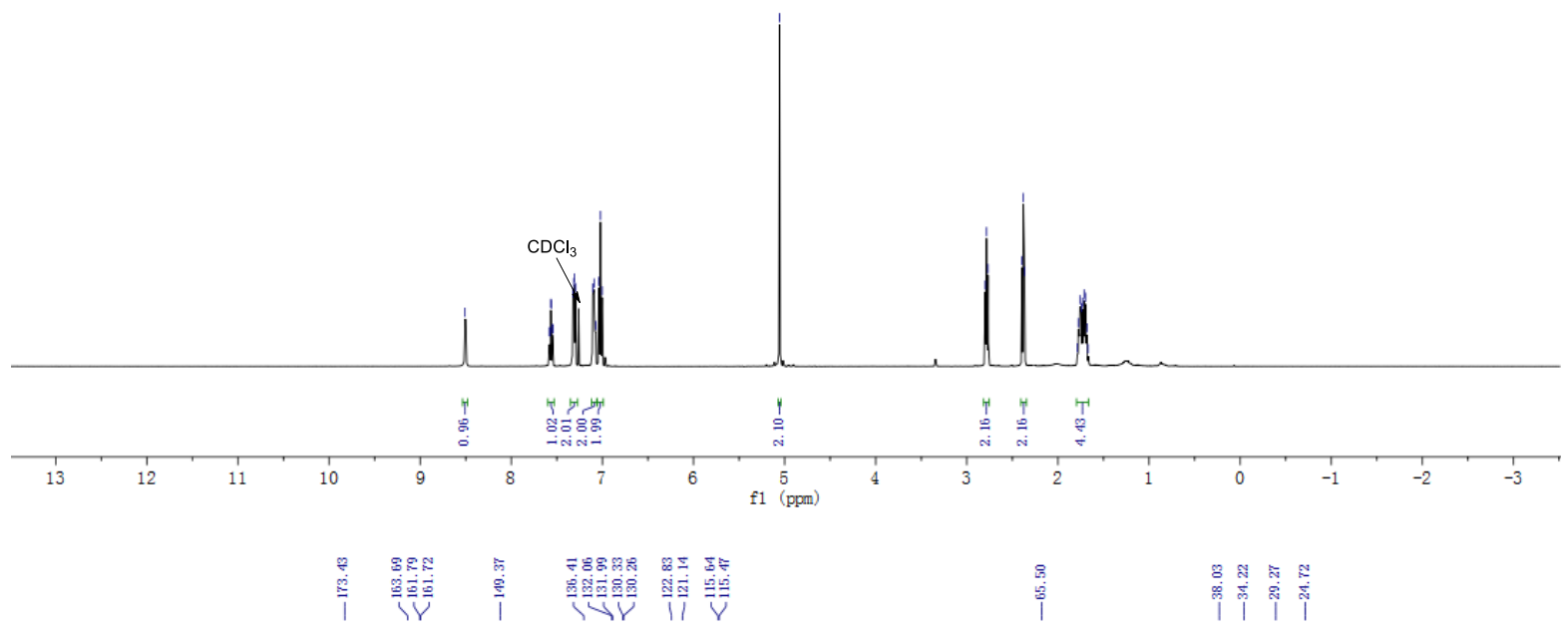<smiles>O=C(CCCCc1ccccn1)OCc1ccc(F)cc1</smiles>

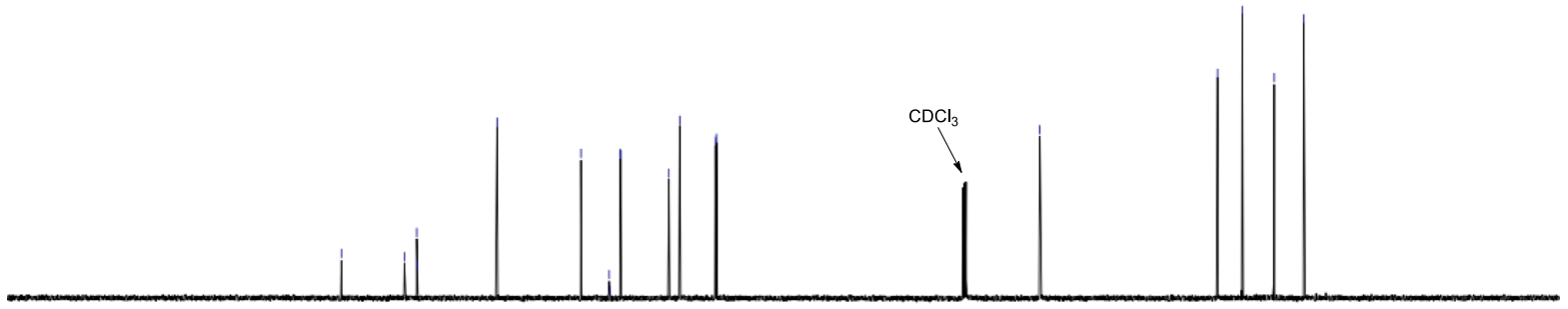

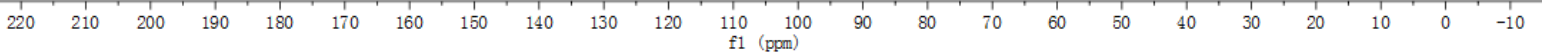




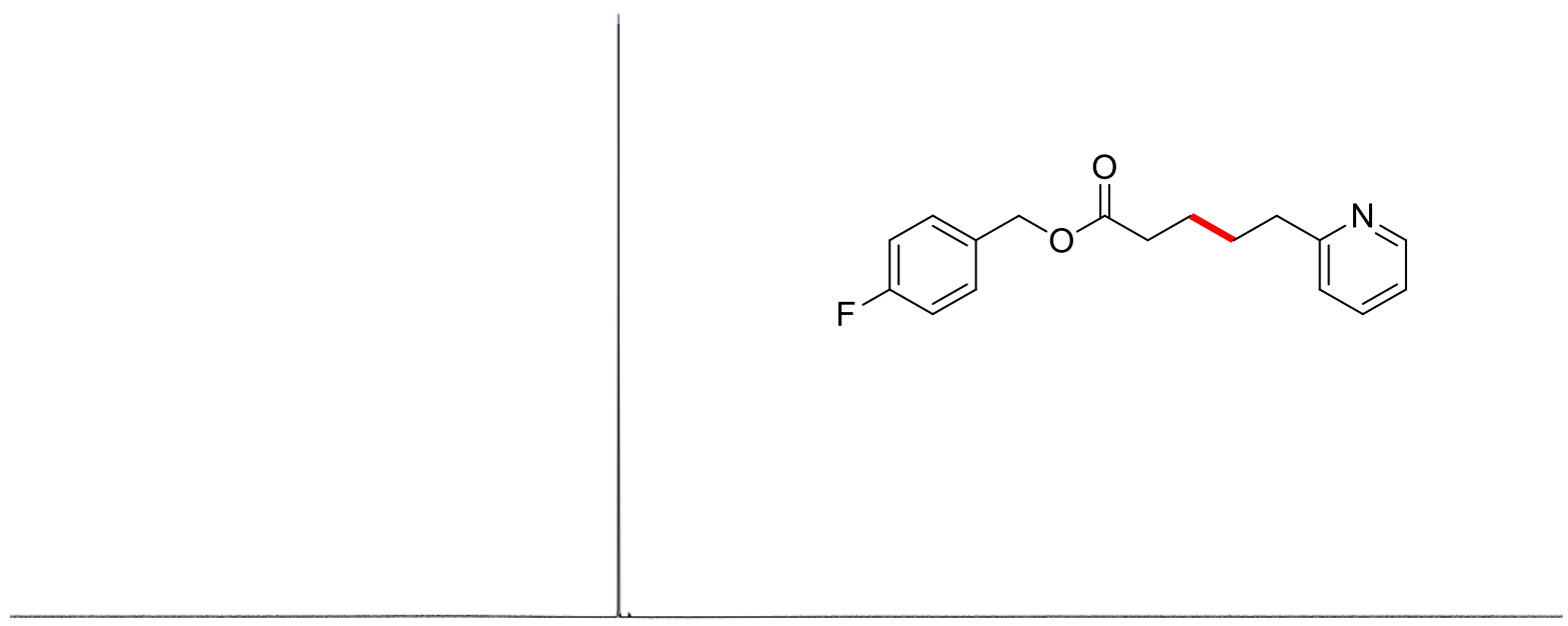

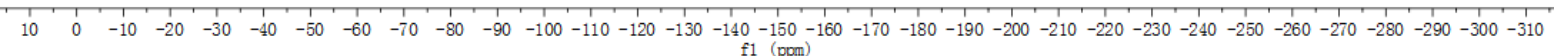


${ }^{1} \mathrm{H}$ NMR $\left(500 \mathrm{MHz}, \mathrm{CDCl}_{3}\right),{ }^{13} \mathrm{C} \mathrm{NMR}\left(126 \mathrm{MHz}, \mathrm{CDCl}_{3}\right)$<smiles>CCOC(=O)CCCCCc1ccccn1</smiles><smiles>CCOC(=O)CCCCCc1ccccn1</smiles>

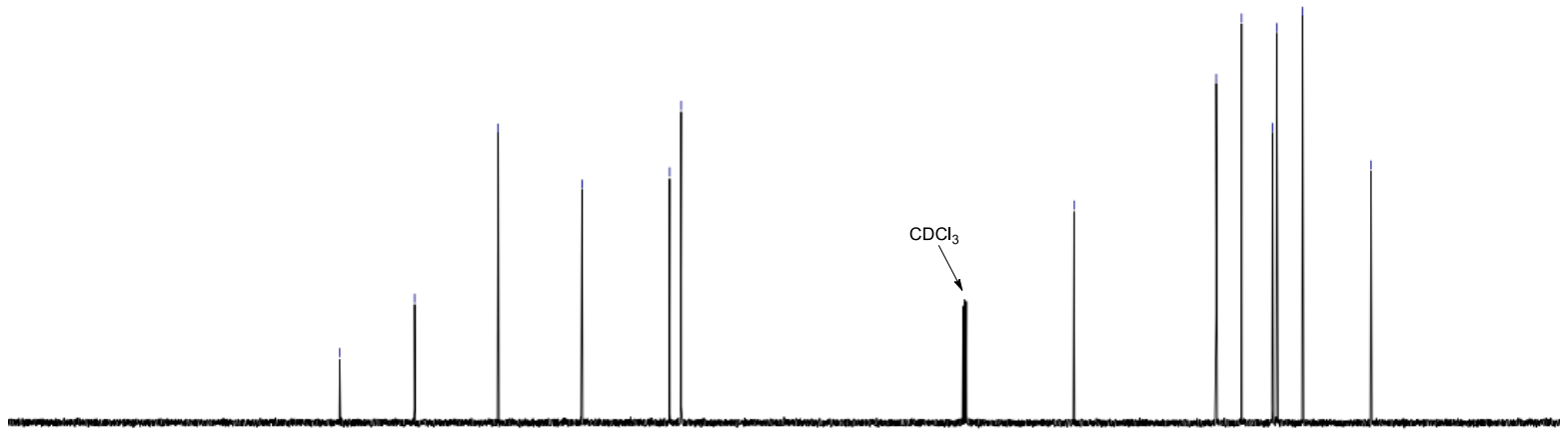

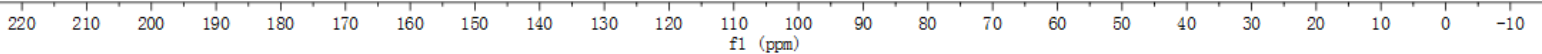


${ }^{1} \mathrm{H}$ NMR $\left(500 \mathrm{MHz}, \mathrm{CDCl}_{3}\right),{ }^{13} \mathrm{C}$ NMR $\left(126 \mathrm{MHz}, \mathrm{CDCl}_{3}\right)$<smiles>CC(C)(C)OCCCCCc1ccccn1</smiles>
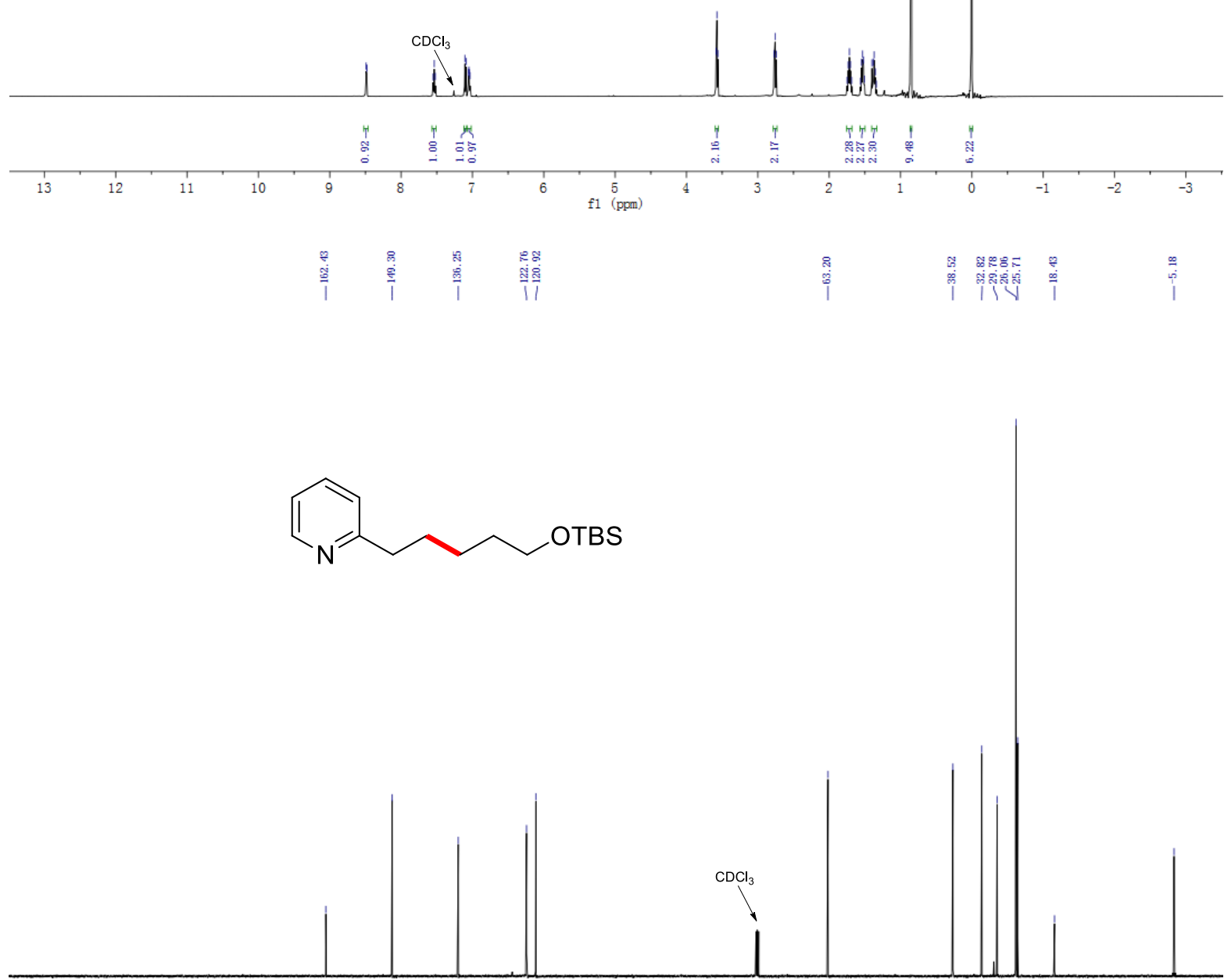

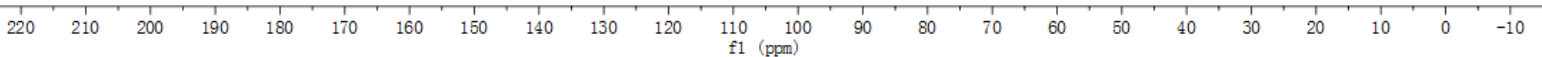


${ }^{1} \mathrm{H} \mathrm{NMR}\left(500 \mathrm{MHz}, \mathrm{CDCl}_{3}\right),{ }^{13} \mathrm{C} \mathrm{NMR}\left(126 \mathrm{MHz}, \mathrm{CDCl}_{3}\right)$<smiles>C#CCCCCCc1ccccn1</smiles>
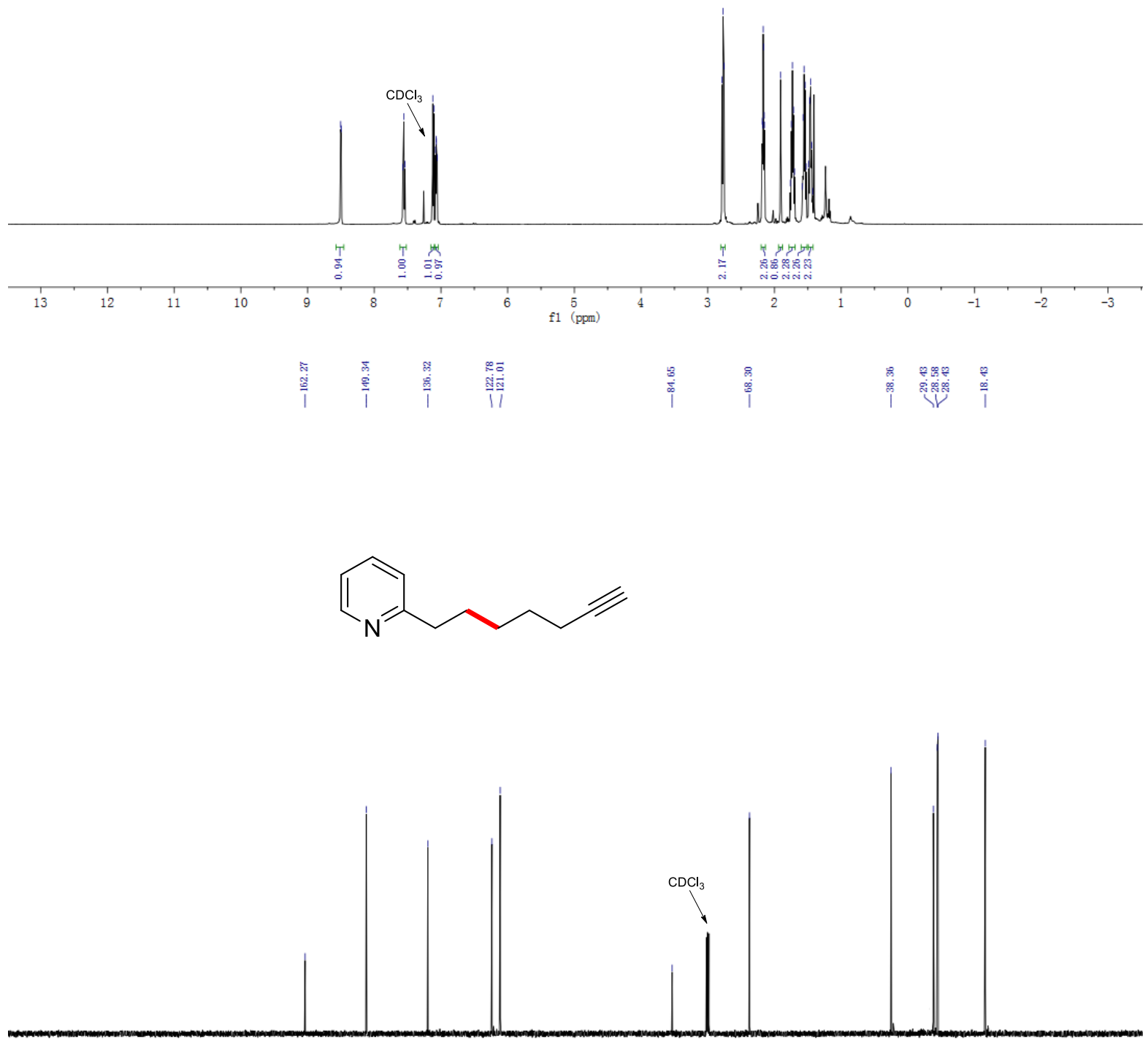

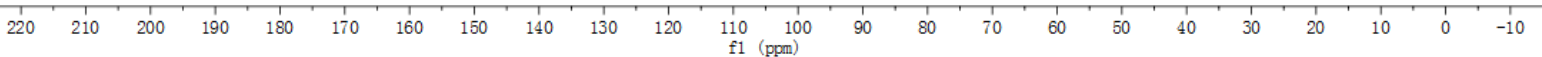




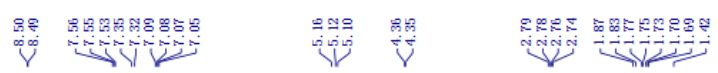

${ }^{1} \mathrm{H} \mathrm{NMR}\left(500 \mathrm{MHz}, \mathrm{CDCl}_{3}\right),{ }^{13} \mathrm{C} \mathrm{NMR}\left(126 \mathrm{MHz}, \mathrm{CDCl}_{3}\right)$<smiles>CC(C)(C)OC(=O)N[C@@H](CCCc1ccccn1)C(=O)OCc1ccccc1</smiles>

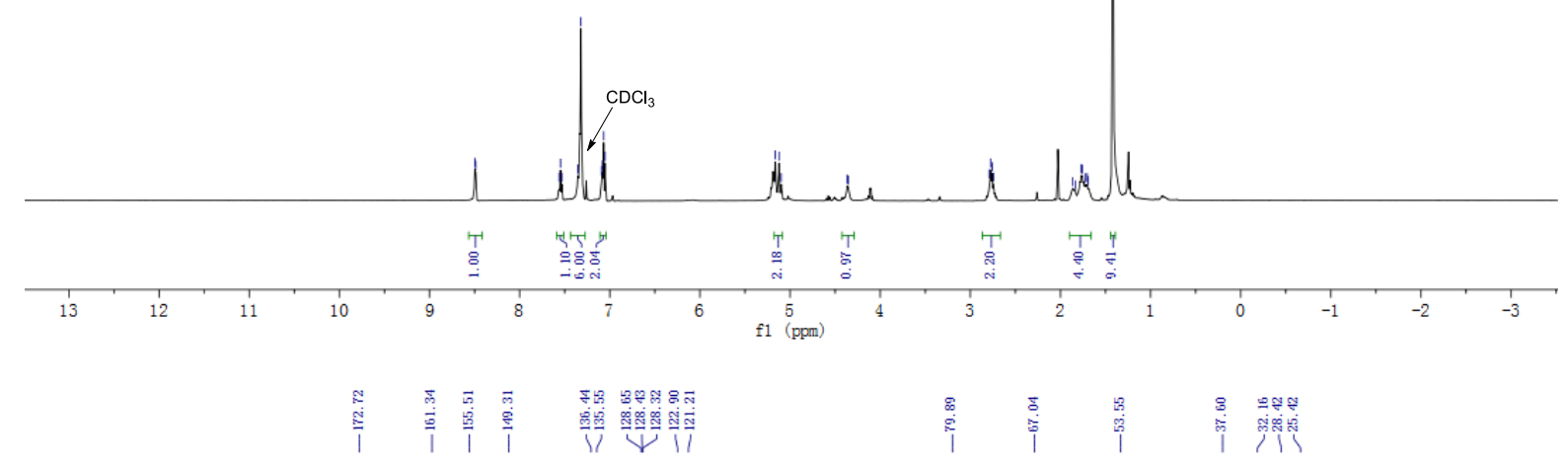<smiles>CC(C)(C)OC(=O)N[C@@H](CCCc1ccccn1)C(=O)OCc1ccccc1</smiles>

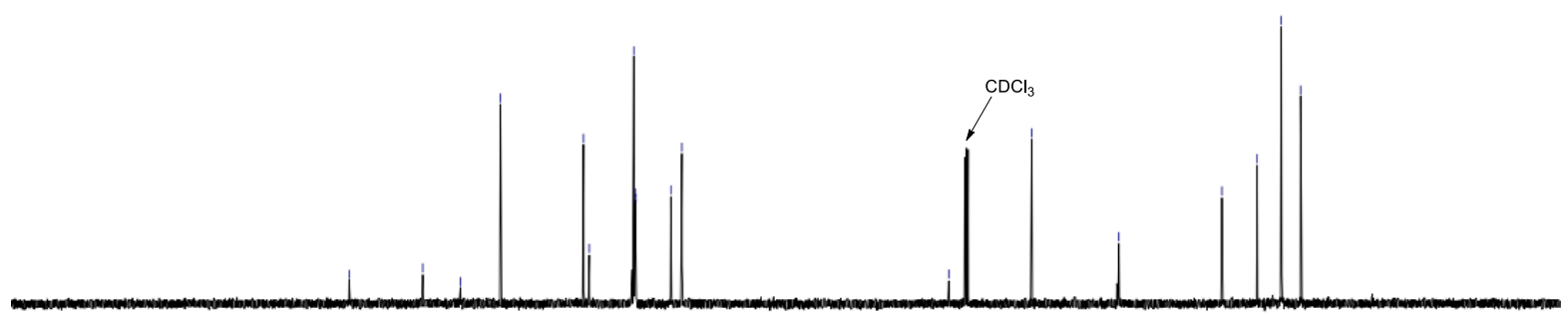

$\begin{array}{lllllllllllllllllllllllll}220 & 210 & 200 & 190 & 180 & 170 & 160 & 150 & 140 & 130 & 120 & 110 & 100 & 90 & 80 & 70 & 60 & 50 & 40 & 30 & 20 & 10 & 0 & -10\end{array}$ 


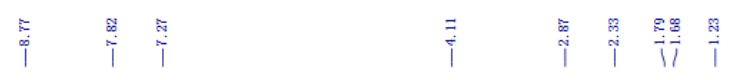

${ }^{1} \mathrm{H}$ NMR $\left(500 \mathrm{MHz}, \mathrm{CDCl}_{3}\right),{ }^{13} \mathrm{C} \mathrm{NMR}\left(126 \mathrm{MHz}, \mathrm{CDCl}_{3}\right),{ }^{19} \mathrm{~F} \mathrm{NMR}\left(376 \mathrm{MHz}, \mathrm{CDCl}_{3}\right)$<smiles>CCOC(=O)CCCCc1ccc(C(F)(F)F)cn1</smiles>
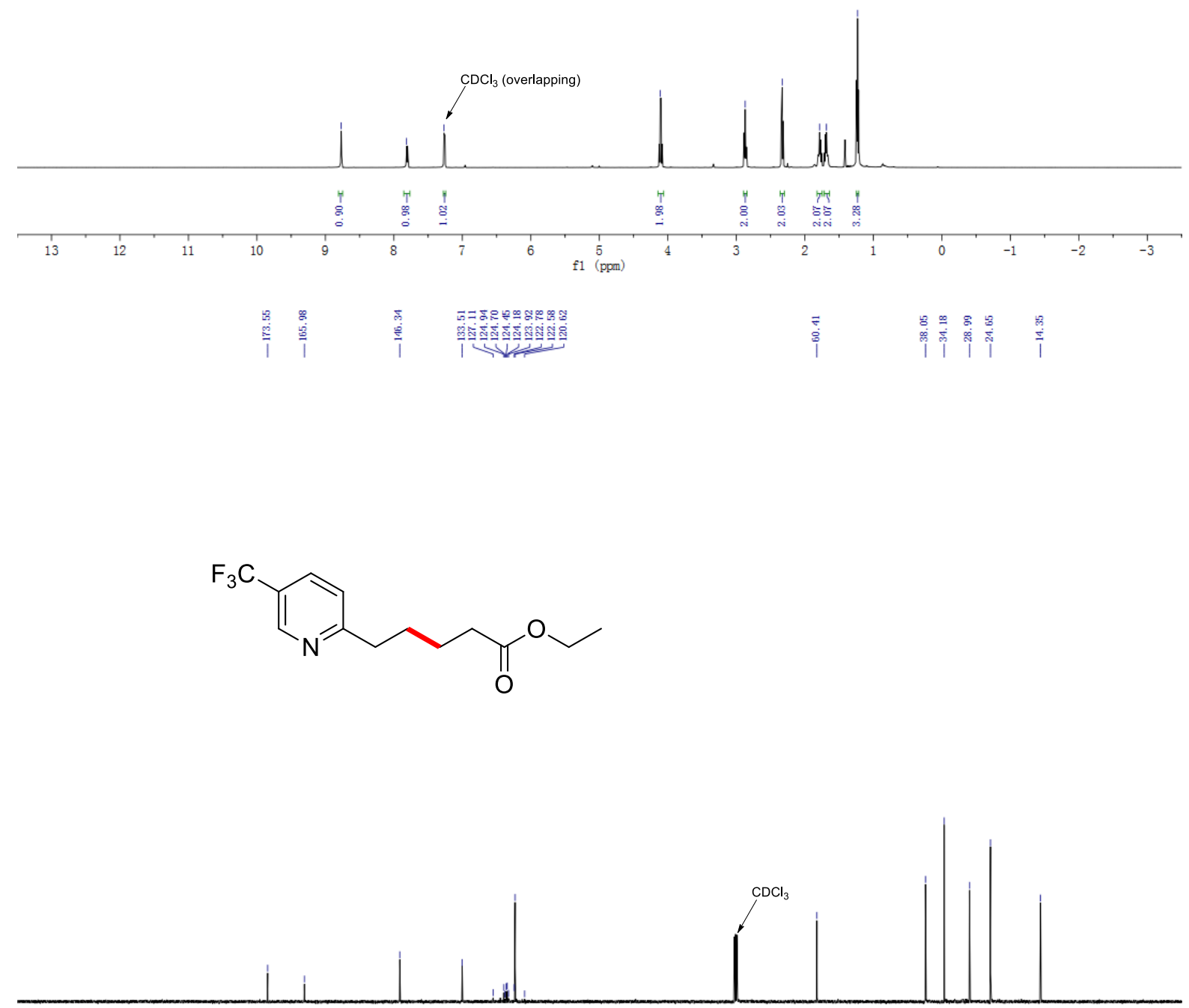

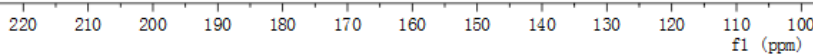




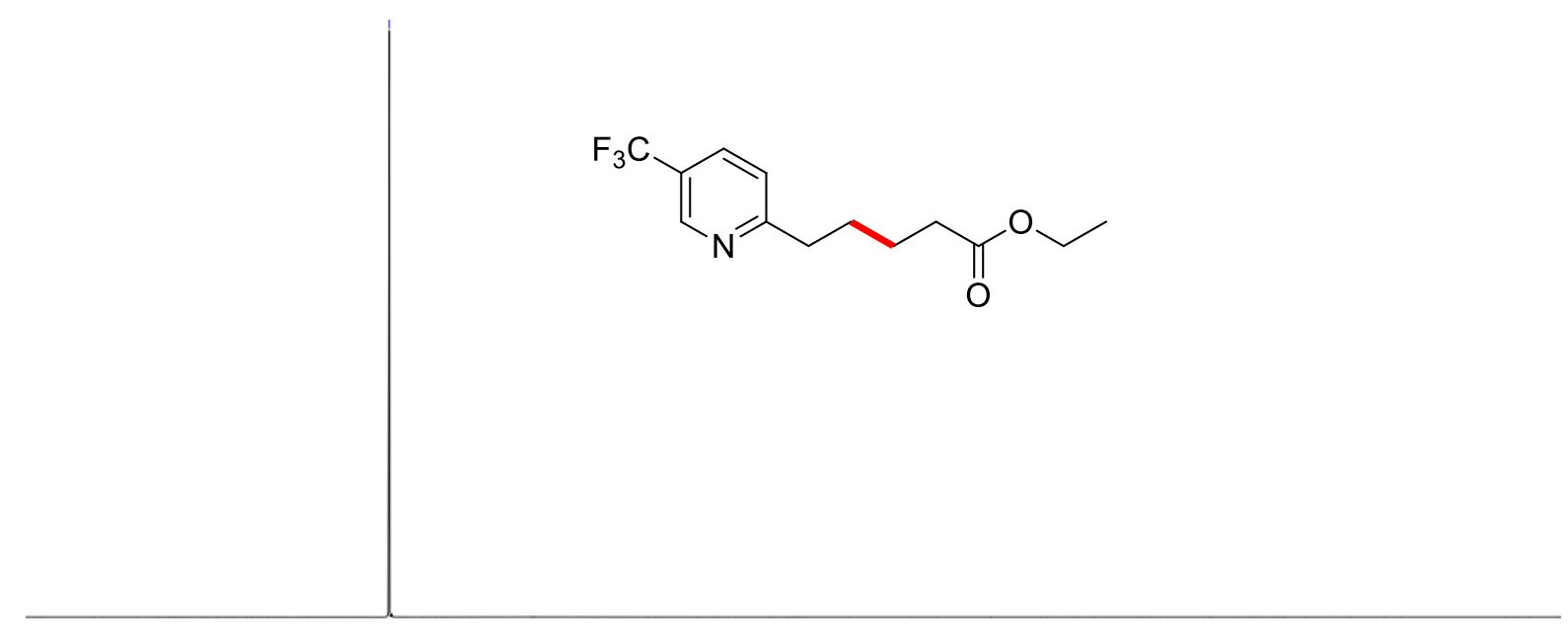

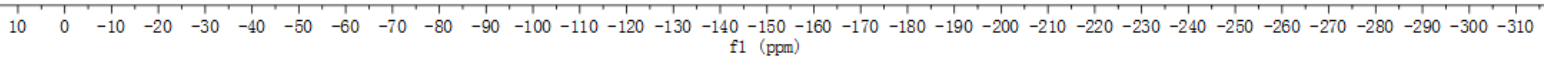




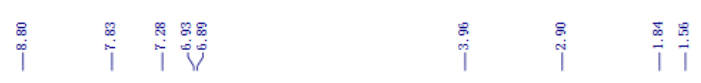

${ }^{1} \mathrm{H} \mathrm{NMR}\left(500 \mathrm{MHz}, \mathrm{CDCl}_{3}\right),{ }^{13} \mathrm{C} \mathrm{NMR}\left(126 \mathrm{MHz}, \mathrm{CDCl}_{3}\right),{ }^{19} \mathrm{~F} \mathrm{NMR}\left(376 \mathrm{MHz}, \mathrm{CDCl}_{3}\right)$<smiles>FC(F)(F)c1ccc(CCCCCOc2ccccc2)nc1</smiles>
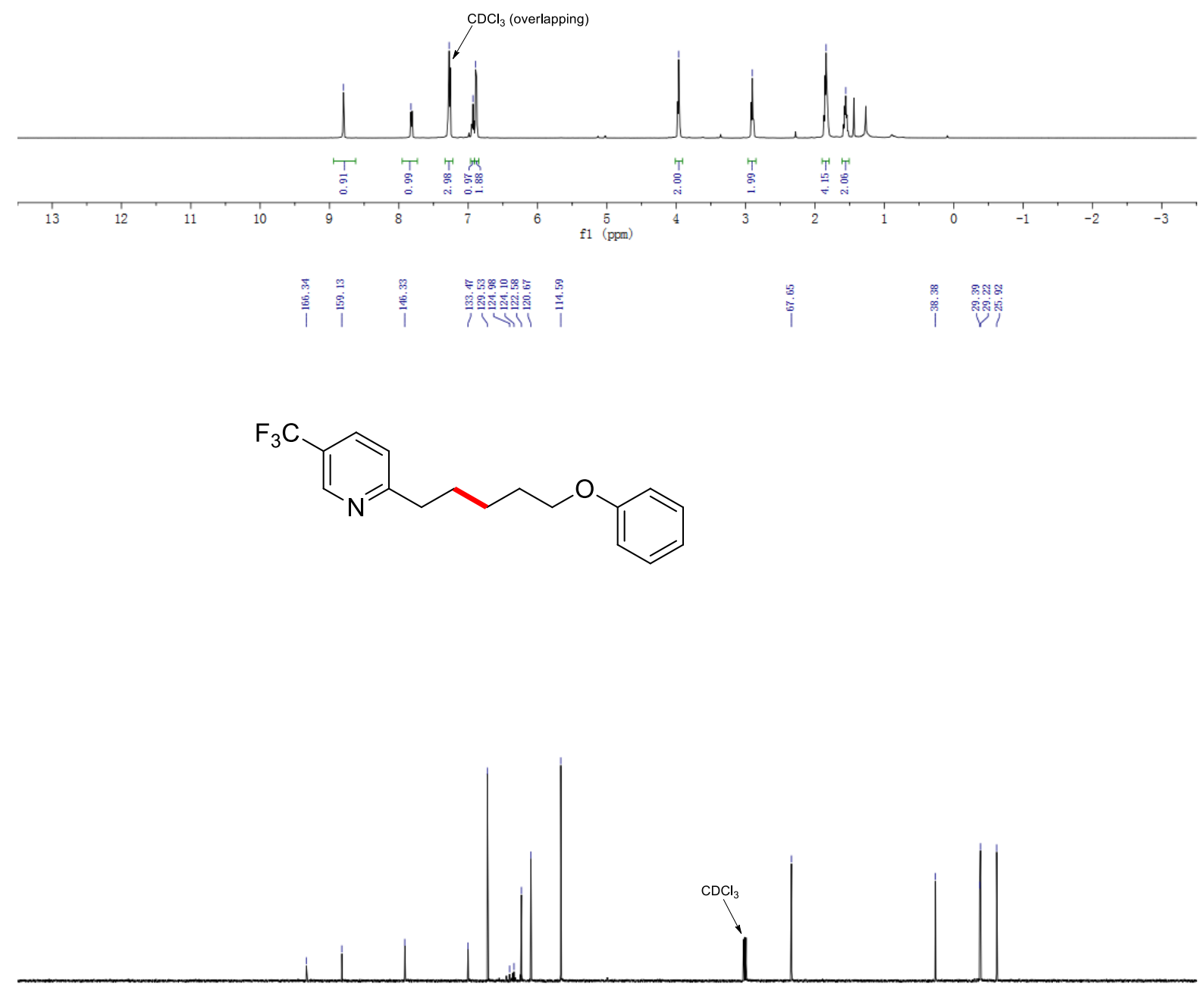

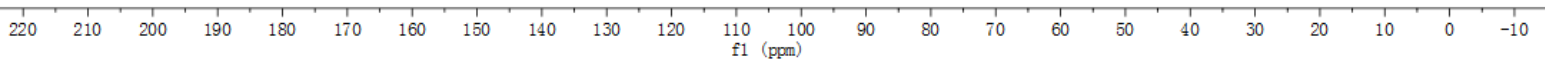




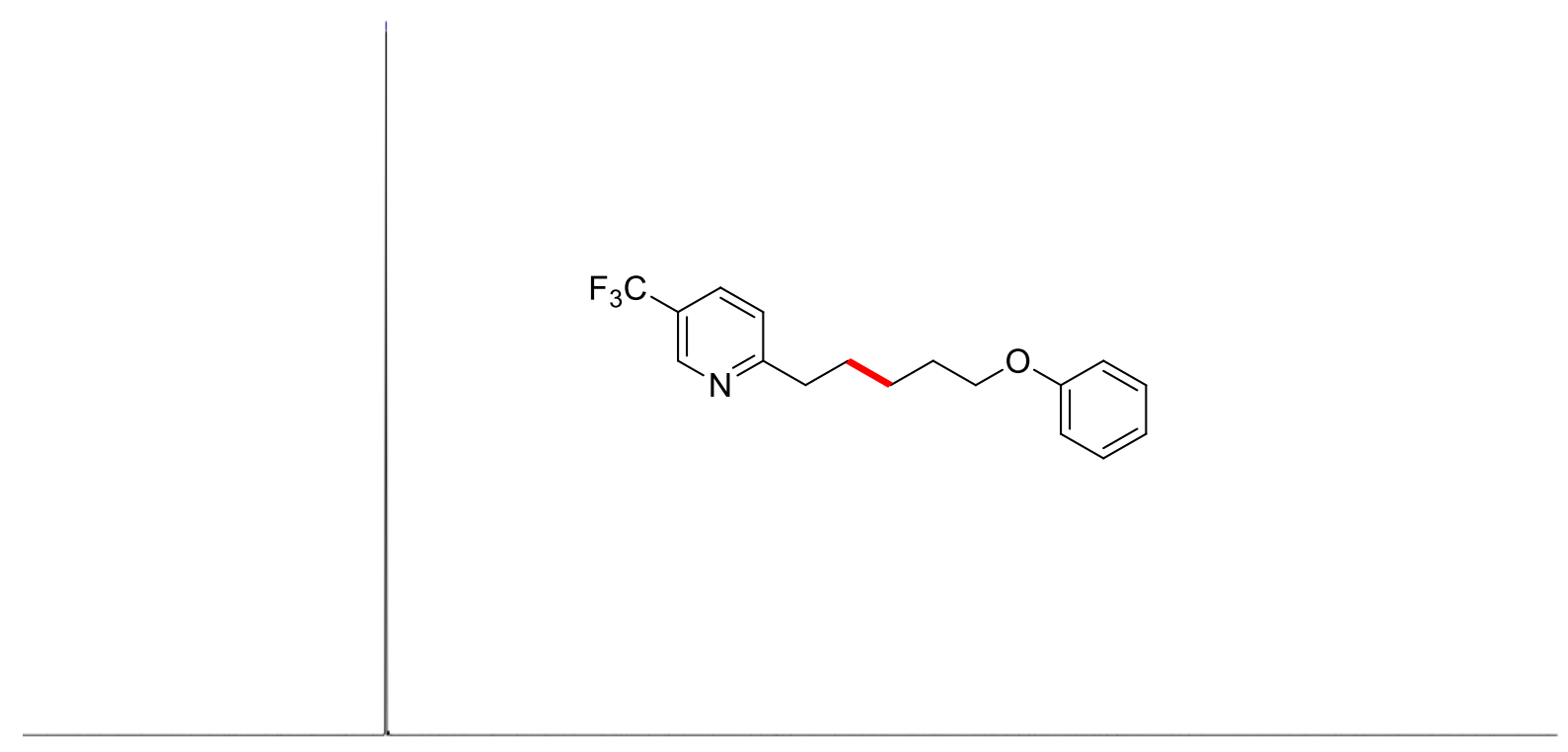

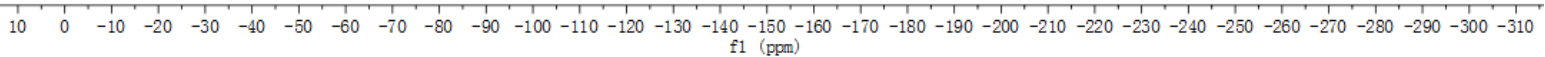


${ }^{1} \mathrm{H}$ NMR $\left(500 \mathrm{MHz}, \mathrm{CDCl}_{3}\right),{ }^{13} \mathrm{C}$ NMR $\left(126 \mathrm{MHz}, \mathrm{CDCl}_{3}\right),{ }^{19} \mathrm{~F} \mathrm{NMR}\left(376 \mathrm{MHz}, \mathrm{CDCl}_{3}\right)$<smiles>O=C(CCCCc1ccc(C(F)(F)F)cn1)Nc1ccc(Cl)c(C(F)(F)F)c1</smiles>

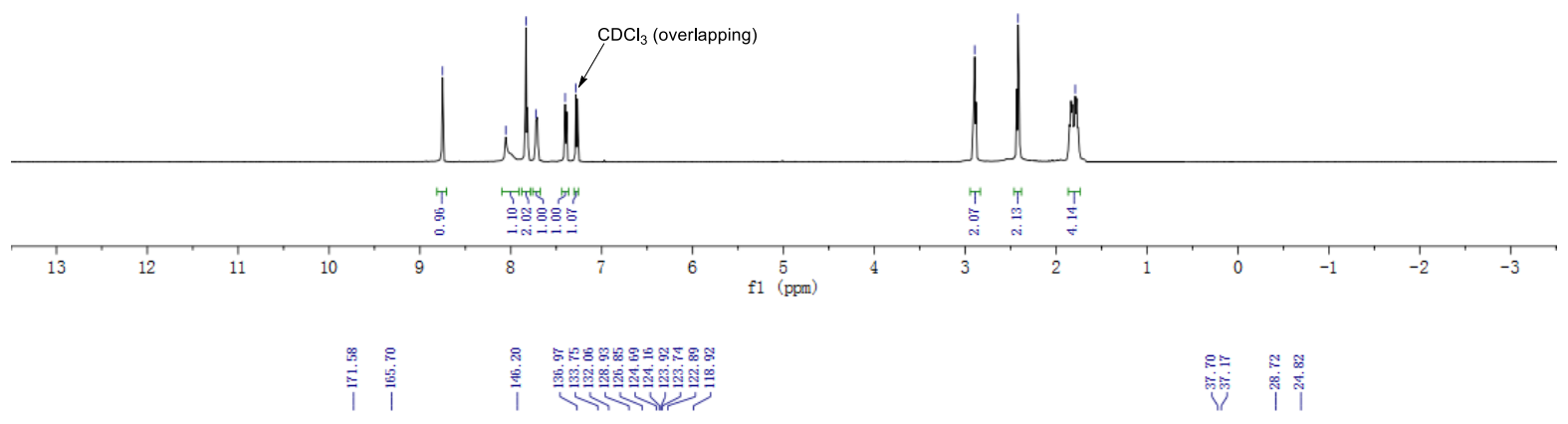<smiles>O=C(CCCCc1ccc(C(F)(F)F)cn1)Nc1ccc(Cl)c(C(F)(F)F)c1</smiles>

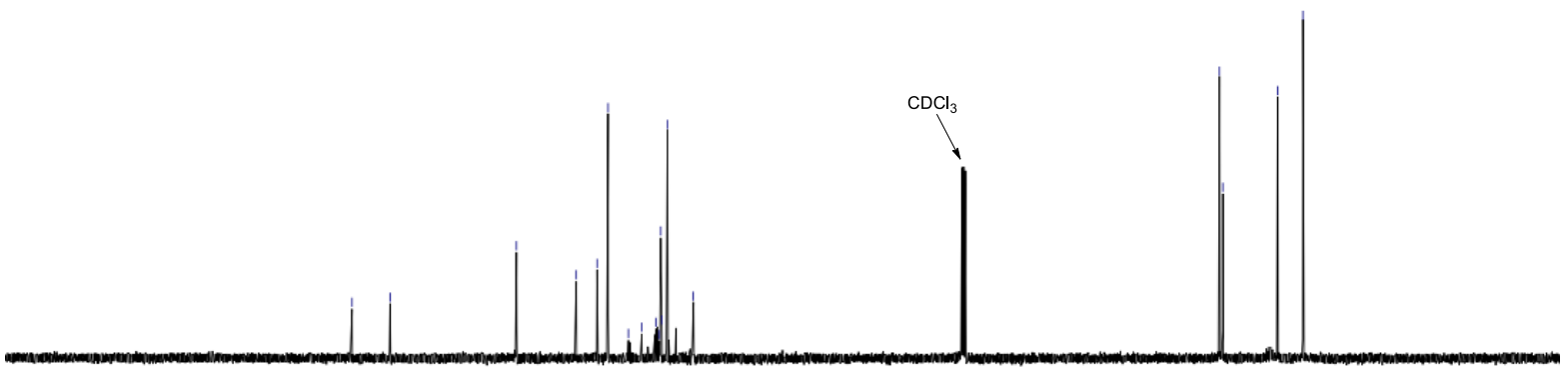

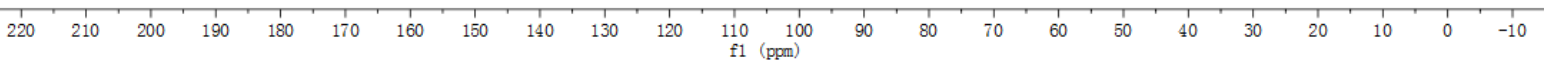




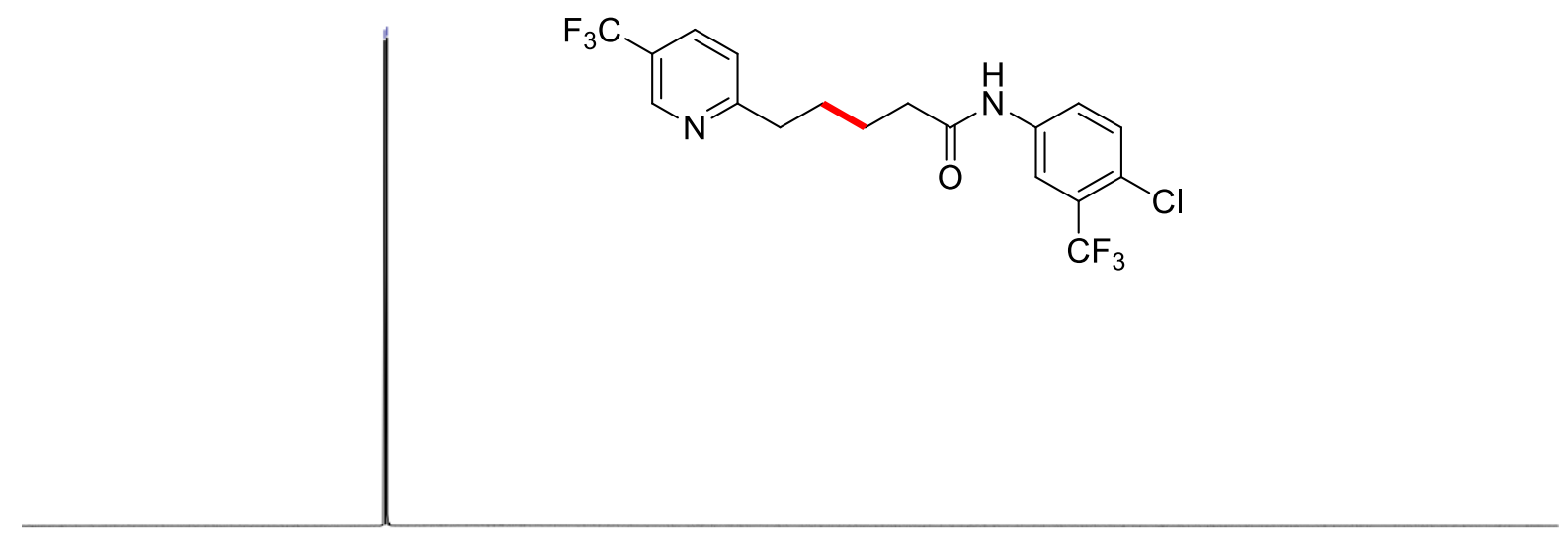

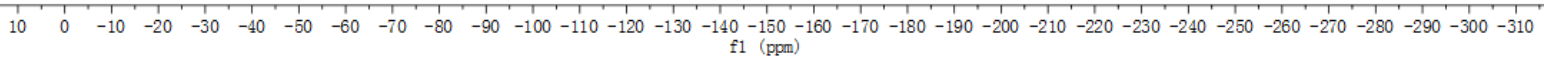


${ }^{1} \mathrm{H}$ NMR $\left(500 \mathrm{MHz}, \mathrm{CDCl}_{3}\right),{ }^{13} \mathrm{C} \mathrm{NMR}\left(126 \mathrm{MHz}, \mathrm{CDCl}_{3}\right)$<smiles>Cc1ncccc1CCCCCOc1ccccc1</smiles>

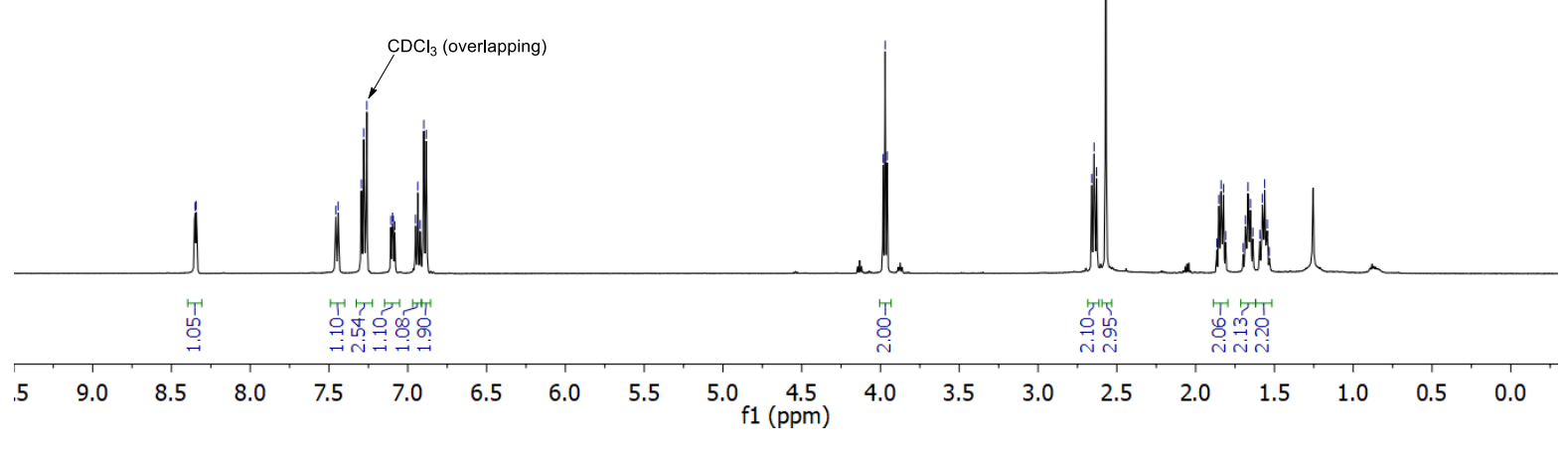

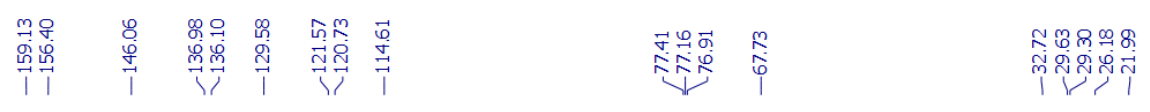<smiles>Cc1ncccc1CCCCCOc1ccccc1</smiles> 


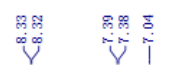

${ }^{1} \mathrm{H}$ NMR $\left(500 \mathrm{MHz}, \mathrm{CDCl}_{3}\right),{ }^{13} \mathrm{C} \mathrm{NMR}\left(126 \mathrm{MHz}, \mathrm{CDCl}_{3}\right)$
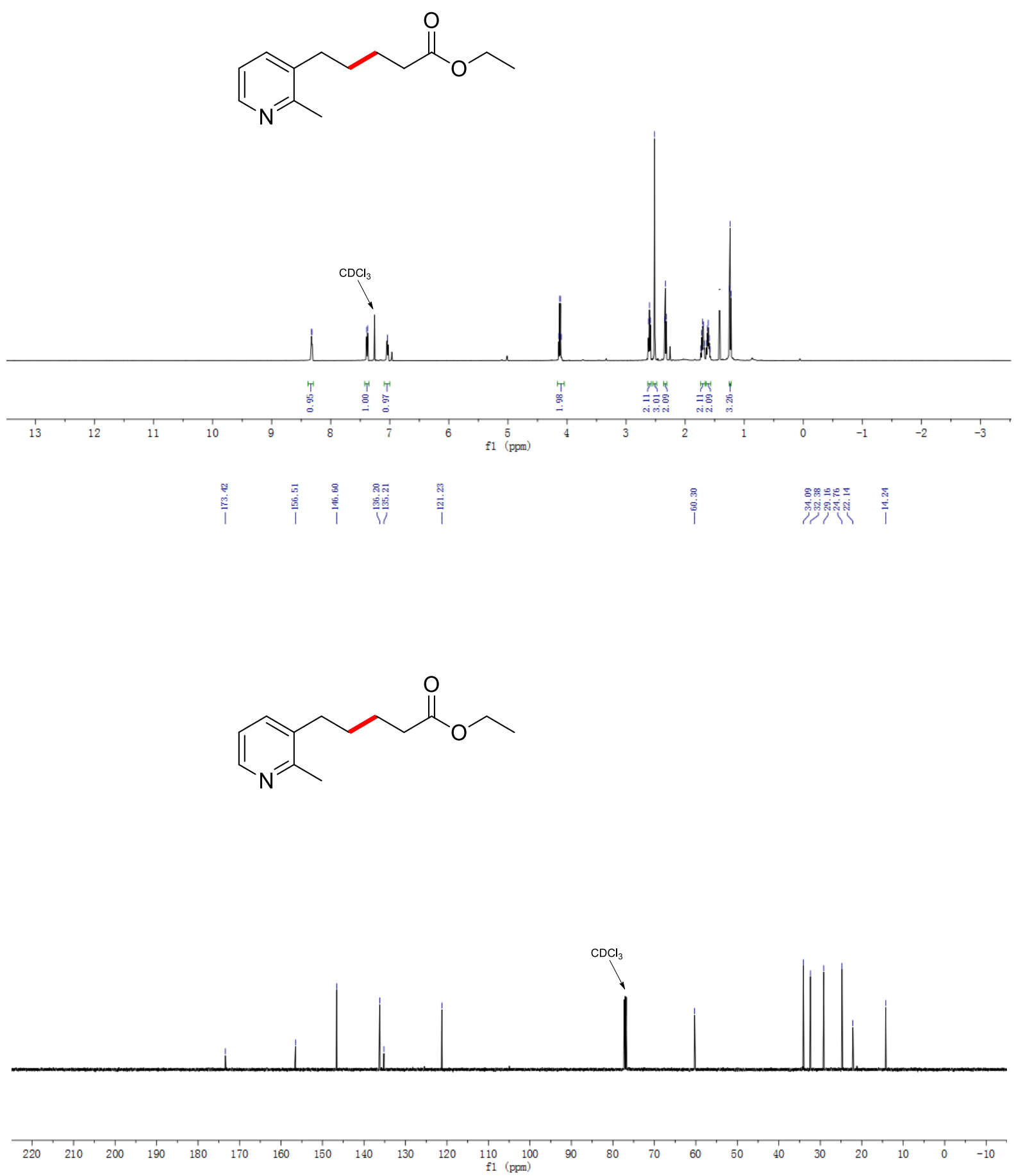

S-103 


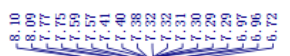

نூர்

${ }^{1} \mathrm{H}$ NMR $\left(500 \mathrm{MHz}, \mathrm{CDCl}_{3}\right),{ }^{13} \mathrm{C}$ NMR (126 MHz, CDCl 3 ), $\left.{ }^{19} \mathrm{~F} \mathrm{NMR} \mathrm{(376} \mathrm{MHz}^{\mathrm{CDCl}} \mathrm{CD}_{3}\right)$<smiles>O=C(CCCCc1ccnc(F)c1)OCC1c2ccccc2-c2ccccc21</smiles>
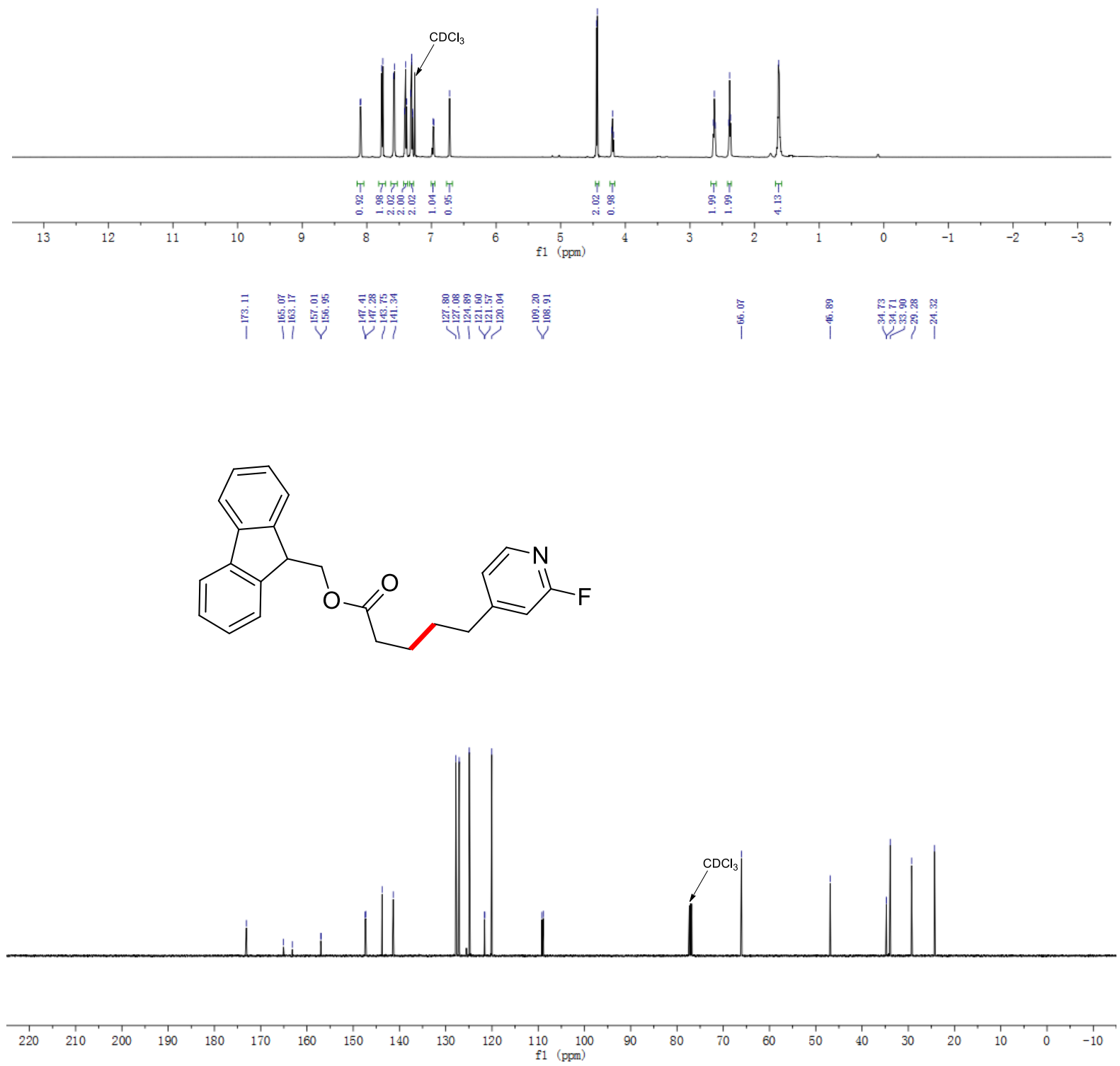

S-104 


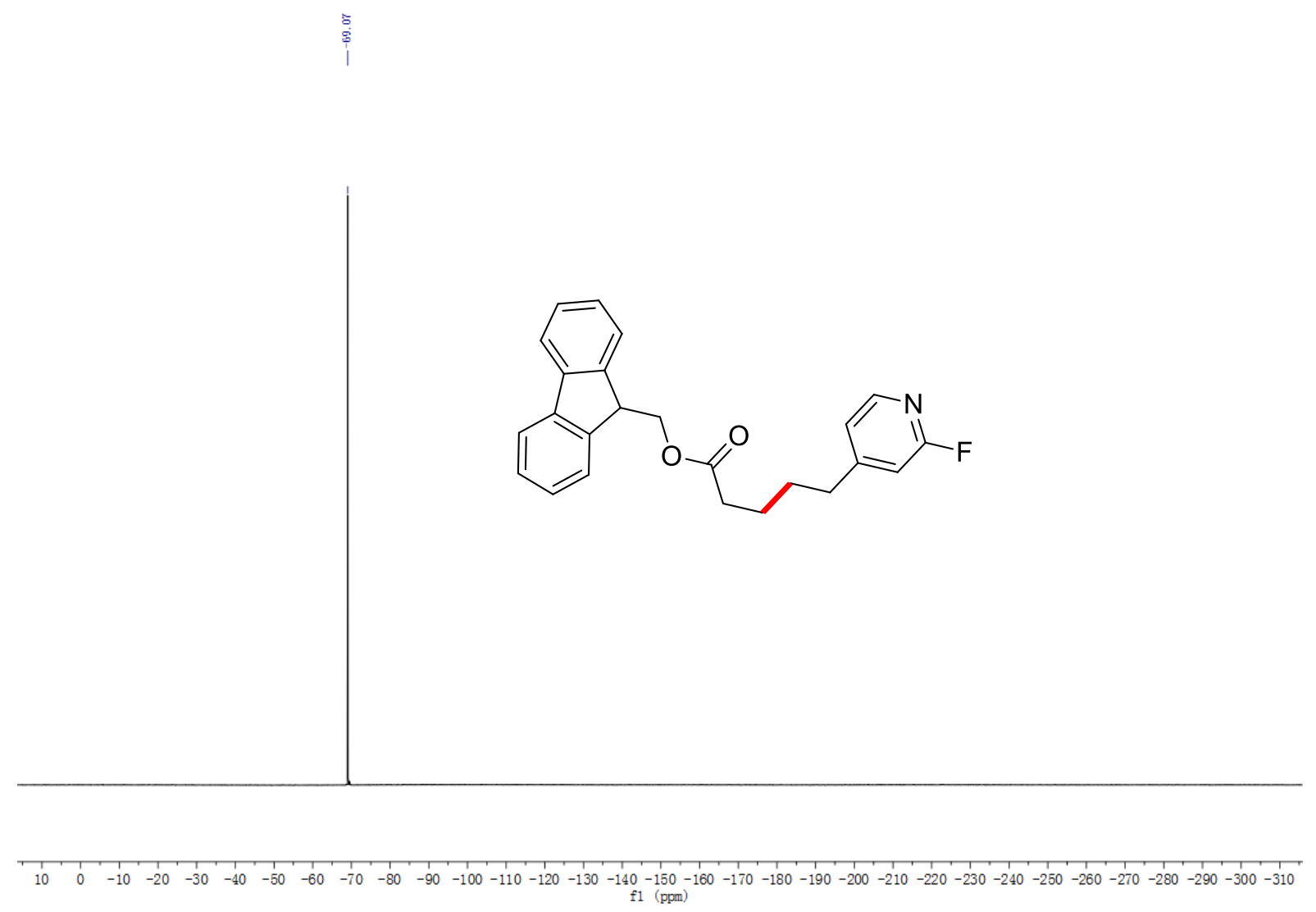




$$
\text { i vivivive }
$$

$\stackrel{i}{i} \underset{i}{\stackrel{i}{i}}$

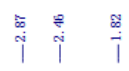

${ }^{1} \mathrm{H} \mathrm{NMR}\left(500 \mathrm{MHz}, \mathrm{CDCl}_{3}\right),{ }^{13} \mathrm{C} \mathrm{NMR}\left(126 \mathrm{MHz} \mathrm{CDCl}_{3}\right),{ }^{19} \mathrm{~F} \mathrm{NMR}\left(376 \mathrm{MHz} \mathrm{CDCl}_{3}\right)$<smiles>O=C(CCCCc1ccc(-c2ccc(OC(F)(F)F)cc2)cn1)OCC1c2ccccc2-c2ccccc21</smiles>
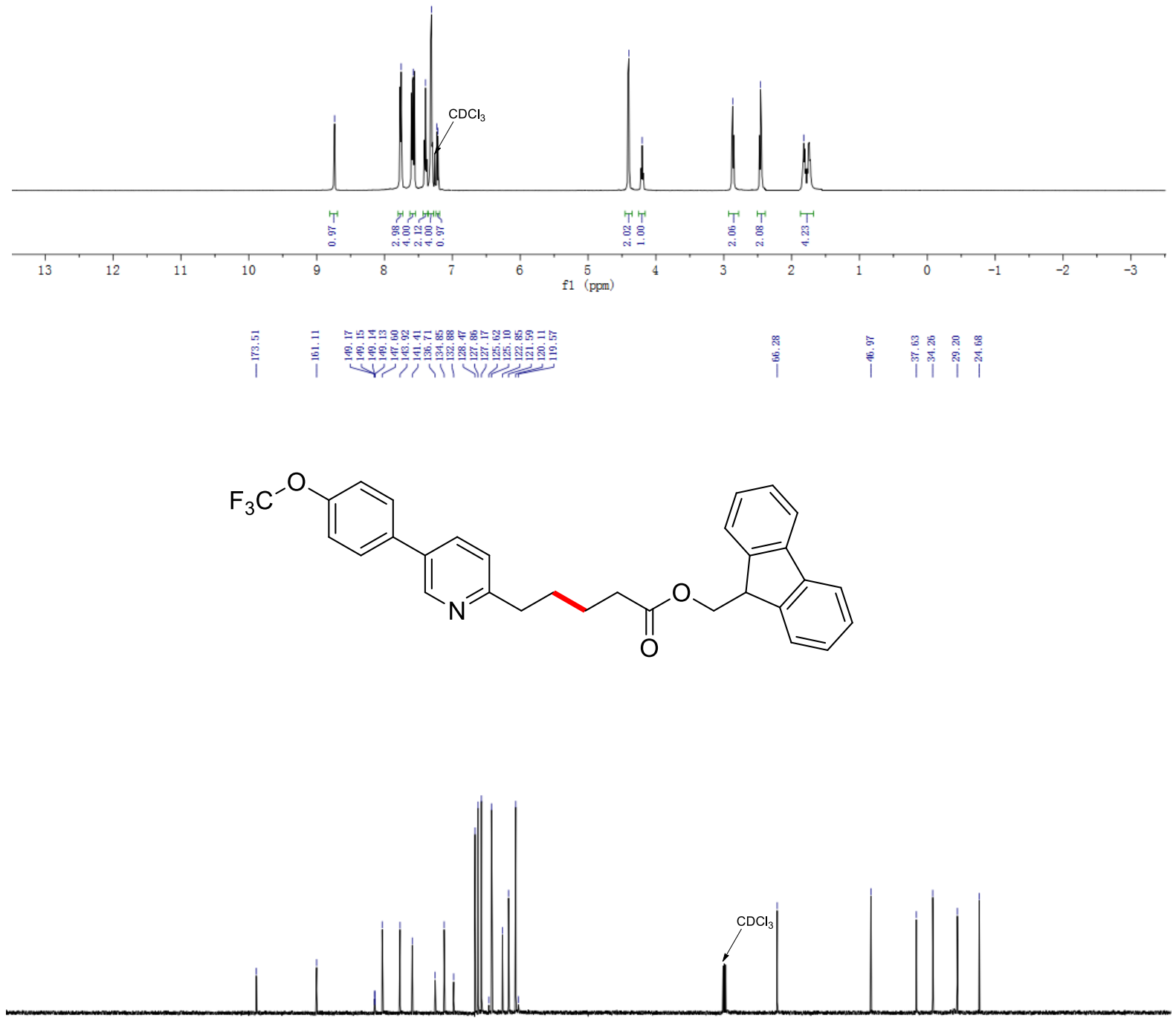

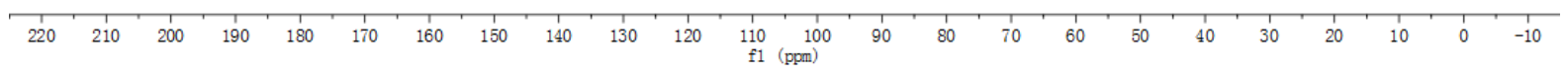

S-106 


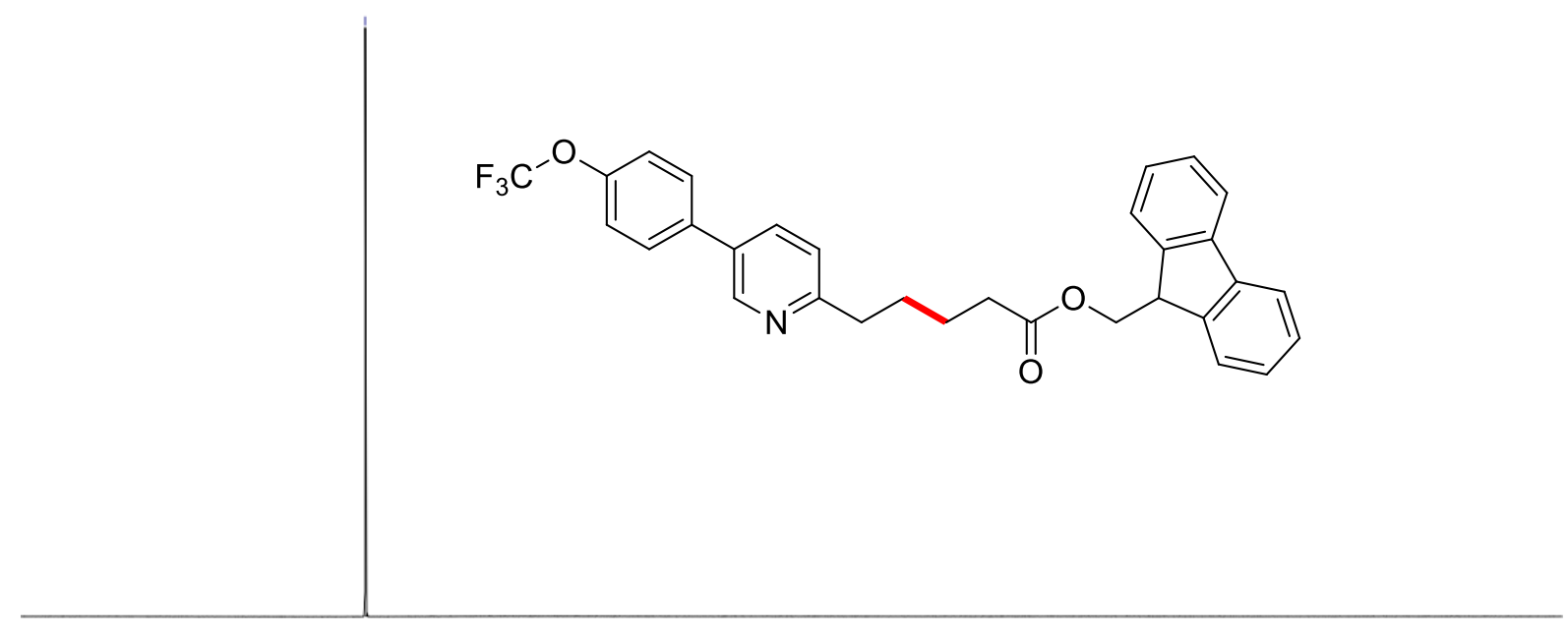

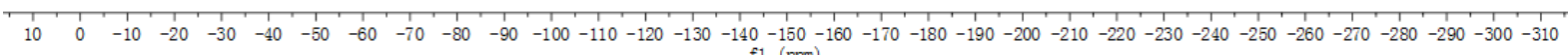
f1 (ppm) 


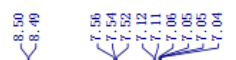

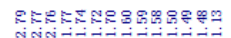

نูingi-itititi

${ }^{1} \mathrm{H}$ NMR $\left(500 \mathrm{MHz}, \mathrm{CDCl}_{3}\right),{ }^{13} \mathrm{C} \mathrm{NMR}\left(126 \mathrm{MHz}, \mathrm{CDCl}_{3}\right)$<smiles>c1ccc(CCC2CCCC2)nc1</smiles>
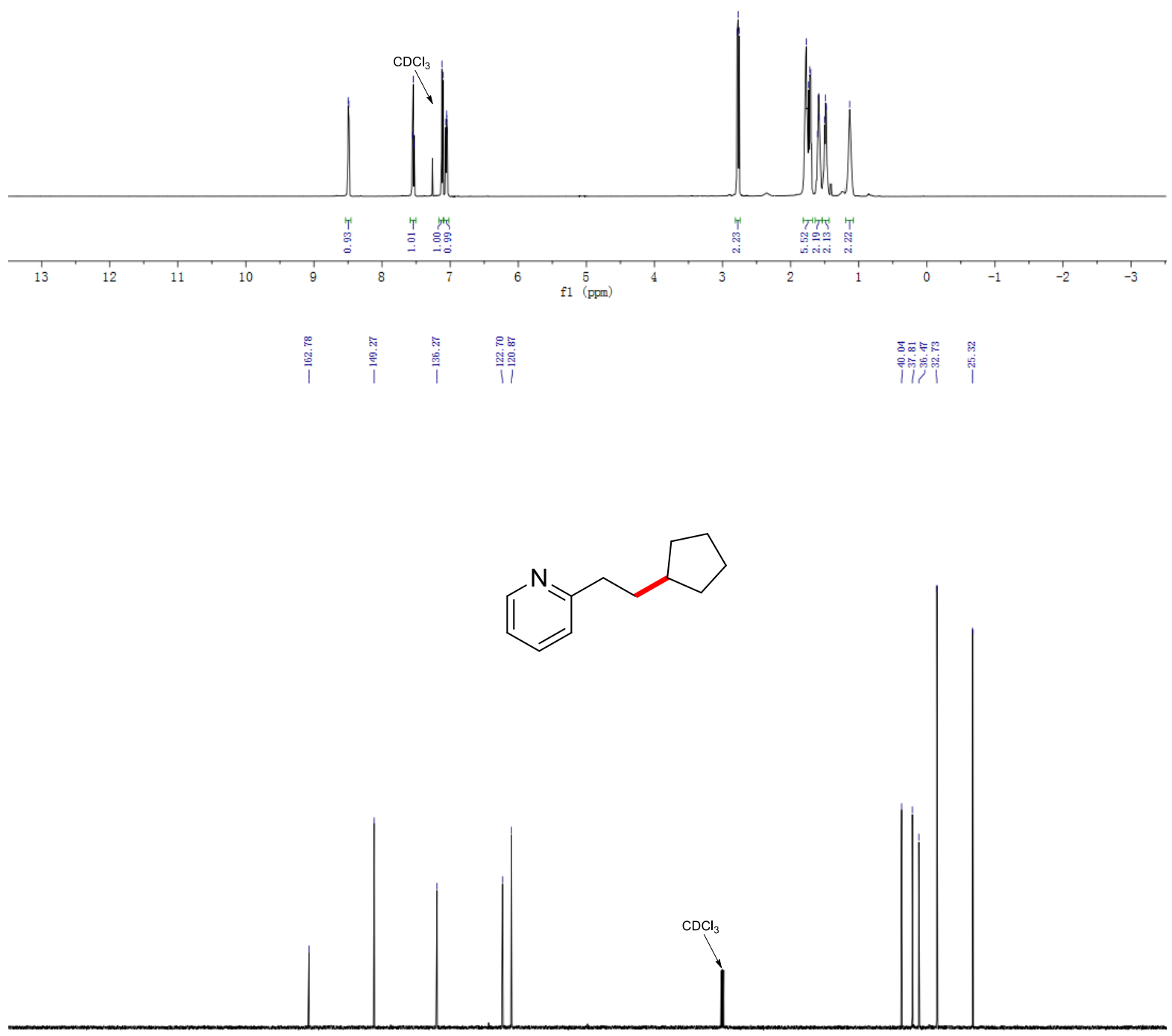

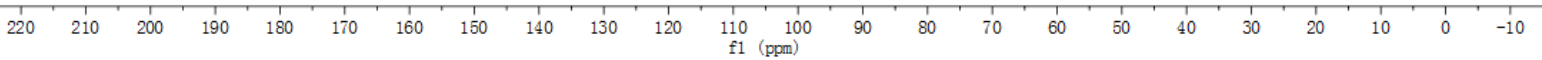


${ }^{1} \mathrm{H} \mathrm{NMR}\left(500 \mathrm{MHz}, \mathrm{CDCl}_{3}\right),{ }^{13} \mathrm{C} \mathrm{NMR}\left(126 \mathrm{MHz}, \mathrm{CDCl}_{3}\right)$
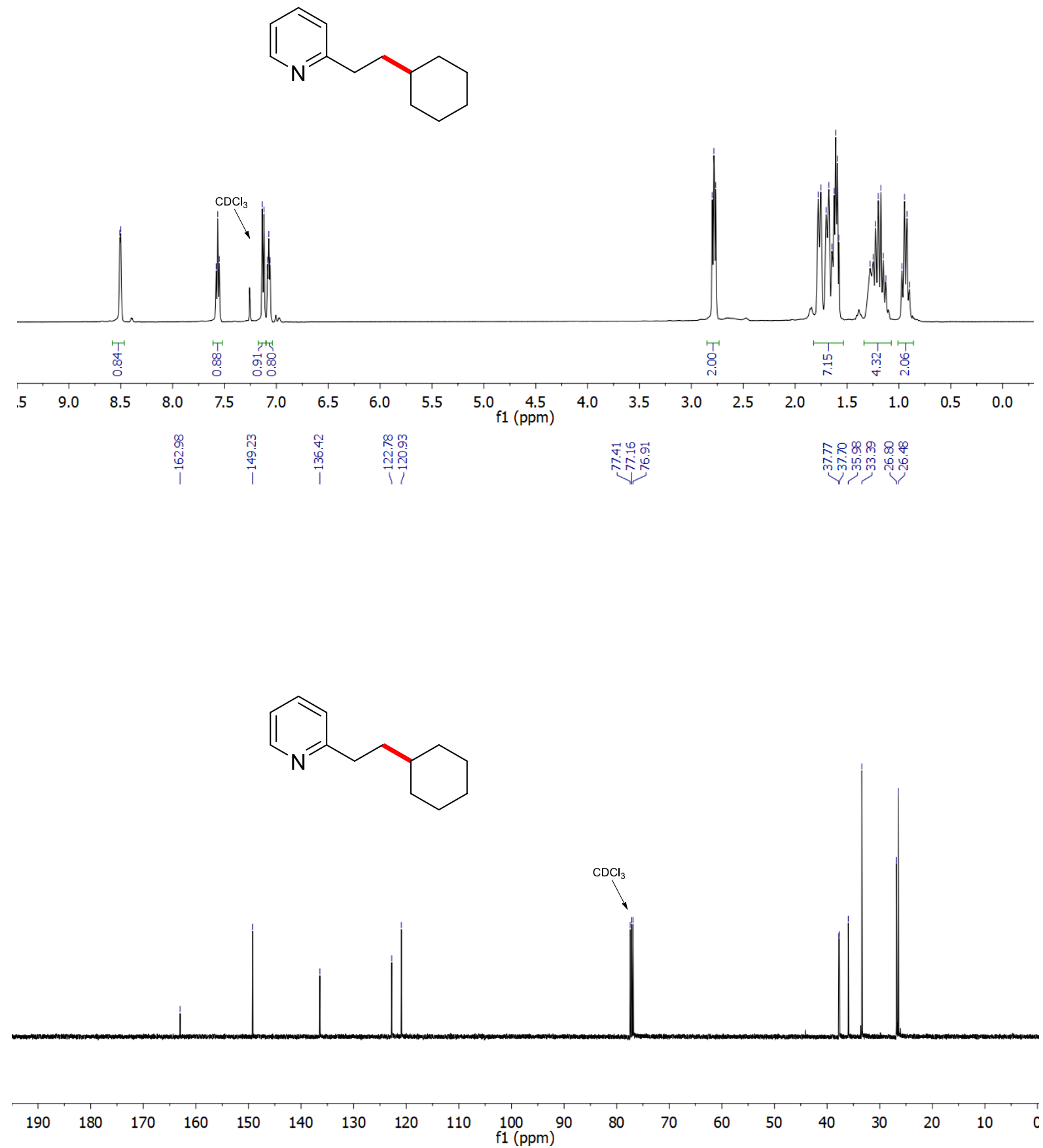
${ }^{1} \mathrm{H}$ NMR $\left(500 \mathrm{MHz}, \mathrm{CDCl}_{3}\right),{ }^{13} \mathrm{C} \mathrm{NMR}\left(126 \mathrm{MHz}, \mathrm{CDCl}_{3}\right)$
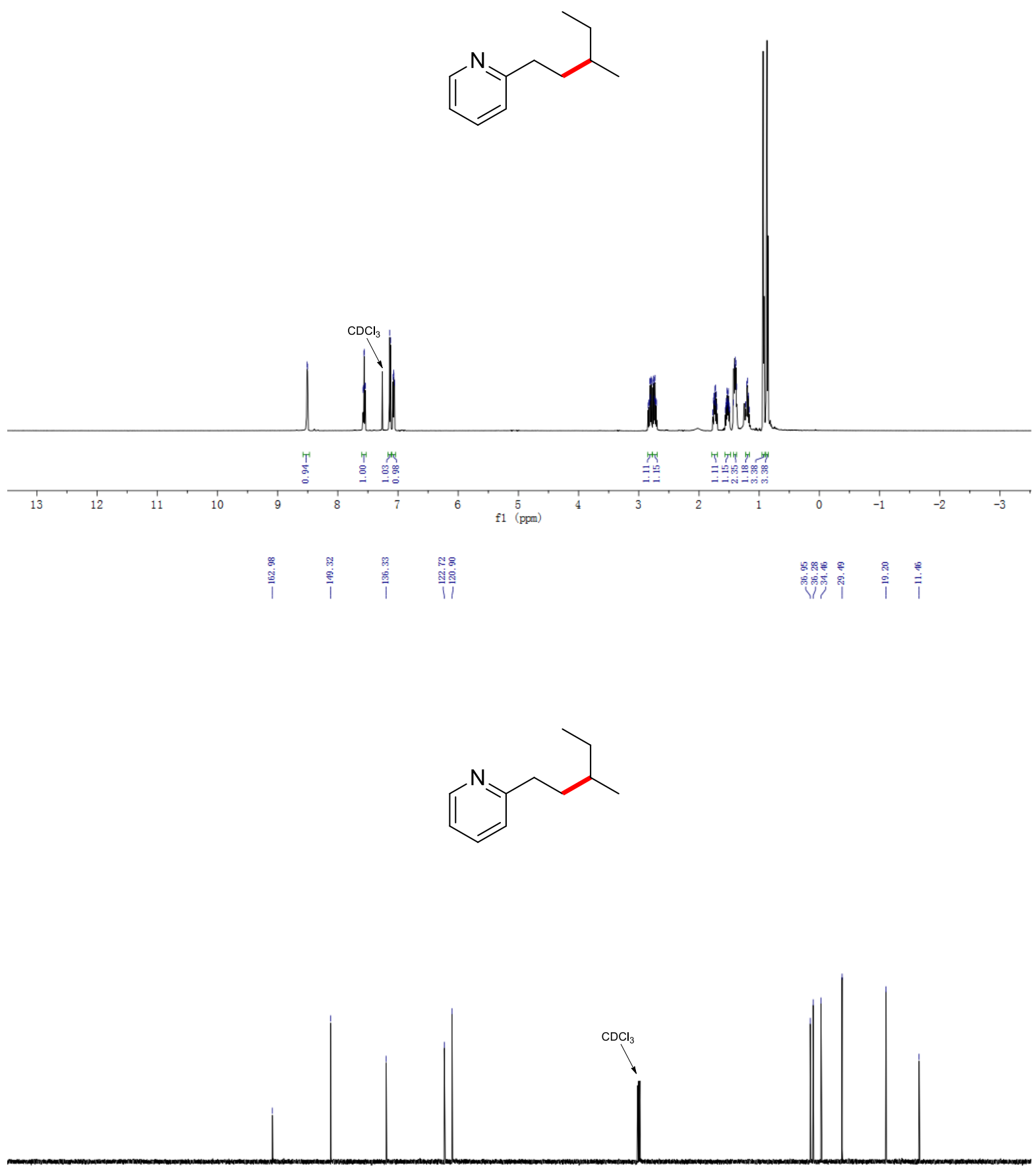

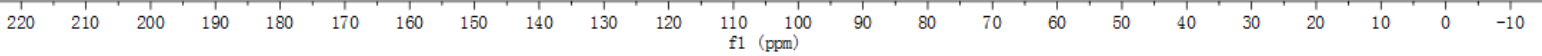


${ }^{1} \mathrm{H} \mathrm{NMR}\left(500 \mathrm{MHz}, \mathrm{CDCl}_{3}\right),{ }^{13} \mathrm{C} \mathrm{NMR}\left(126 \mathrm{MHz}, \mathrm{CDCl}_{3}\right)$<smiles>c1ccc(CCC2CCOCC2)nc1</smiles>

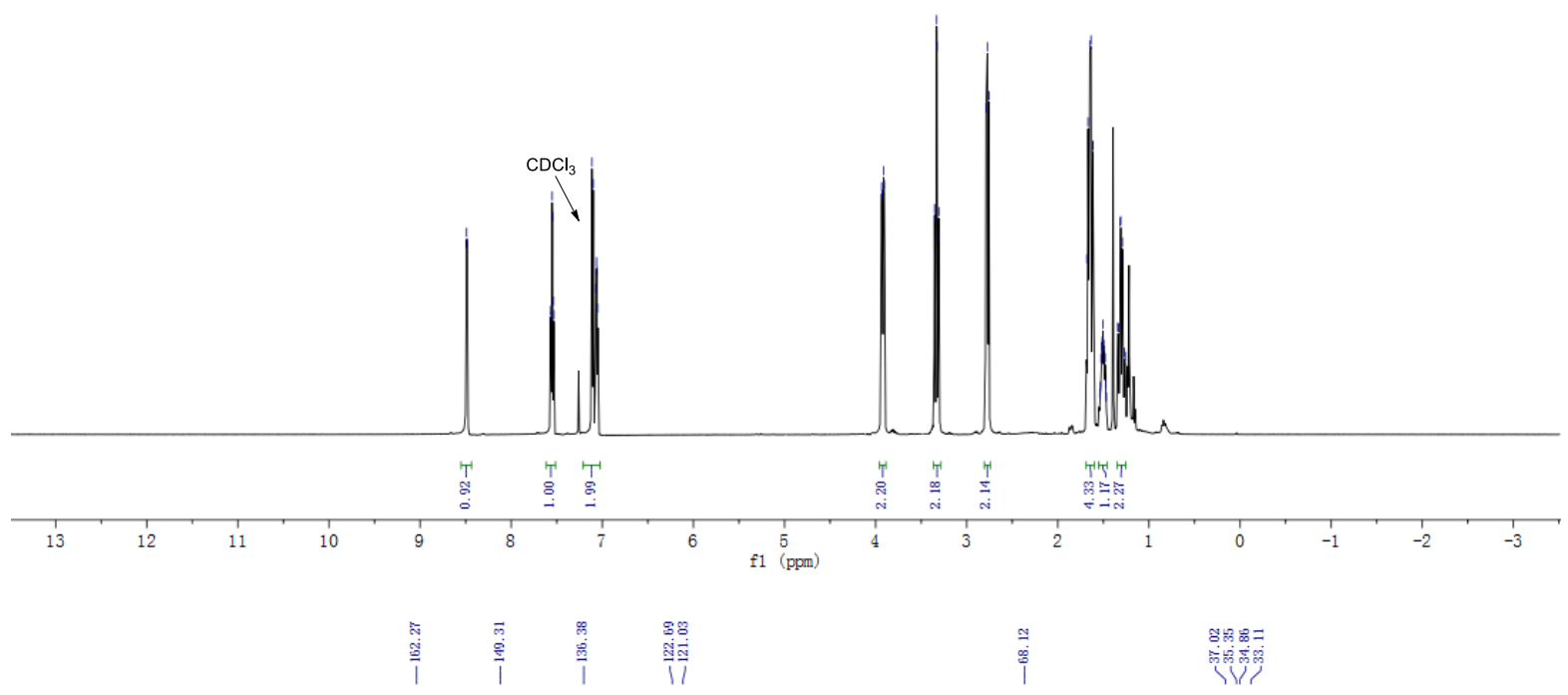<smiles>c1ccc(CCC2CCOCC2)nc1</smiles>

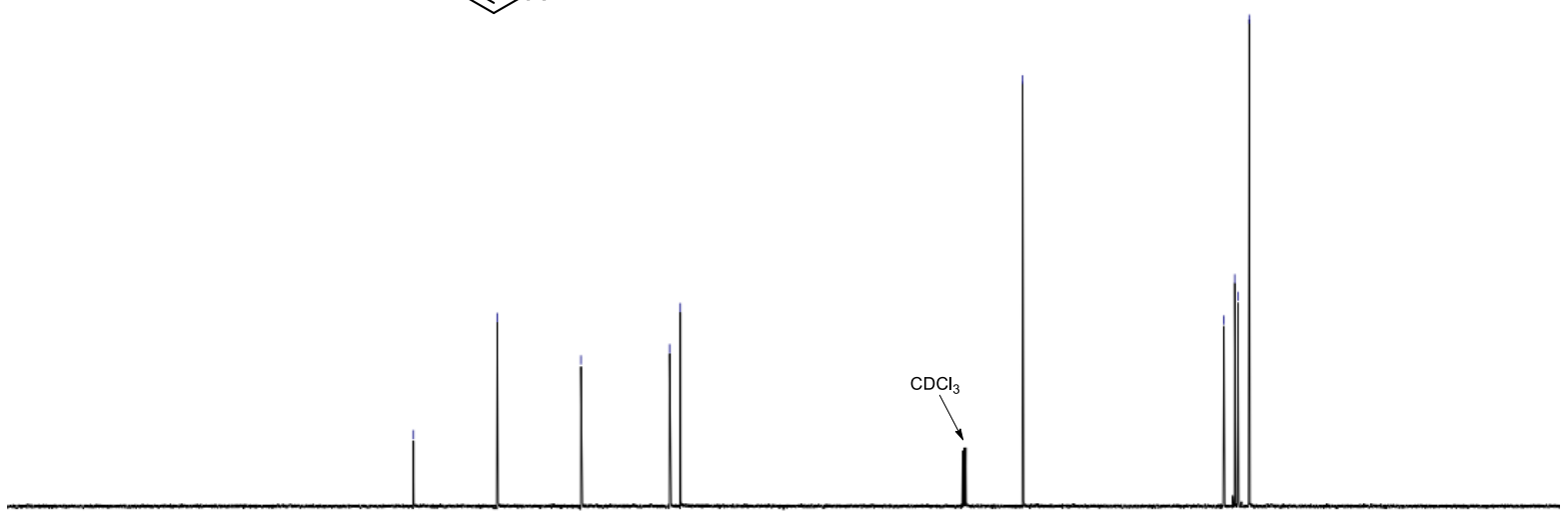

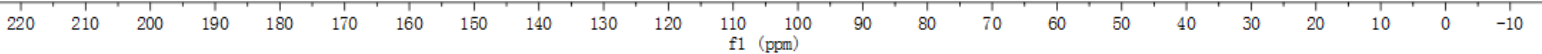


${ }^{1} \mathrm{H} \mathrm{NMR}\left(500 \mathrm{MHz}, \mathrm{CDCl}_{3}\right),{ }^{13} \mathrm{C} \mathrm{NMR}\left(126 \mathrm{MHz}, \mathrm{CDCl}_{3}\right)$
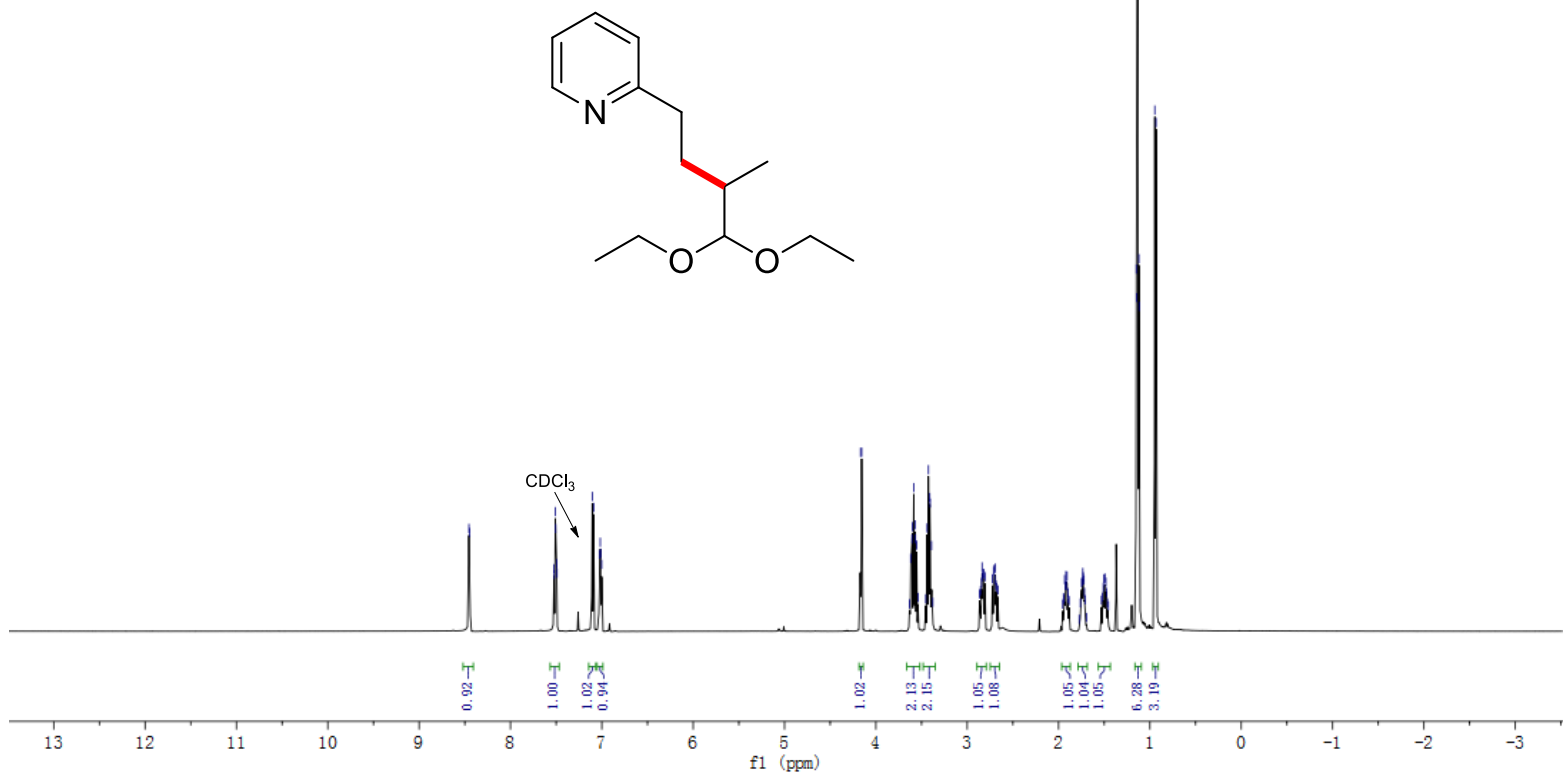

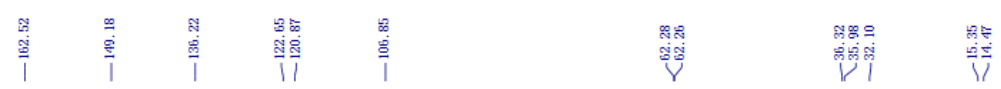
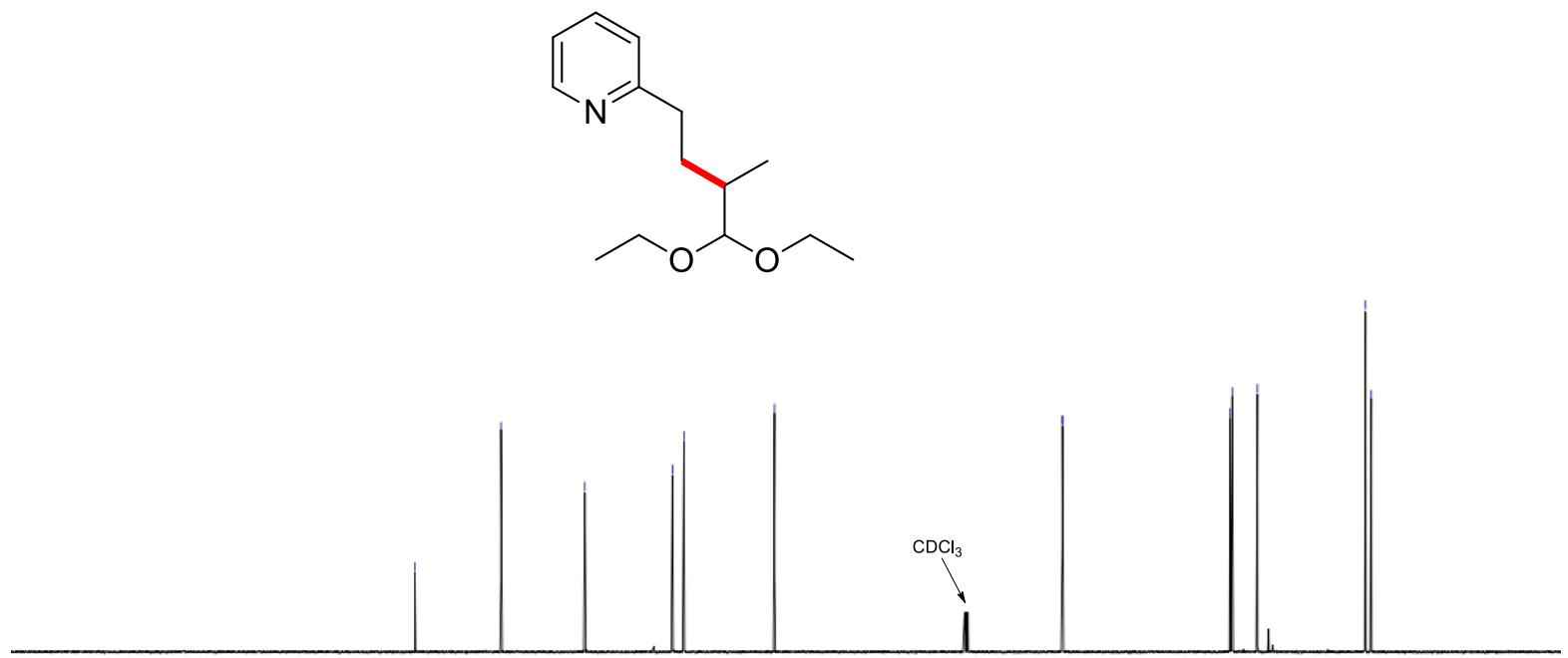

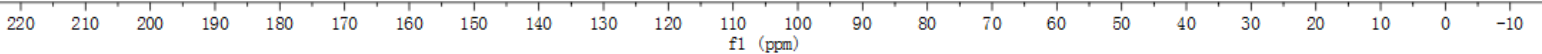


${ }^{1} \mathrm{H} \mathrm{NMR}\left(500 \mathrm{MHz}, \mathrm{CDCl}_{3}\right),{ }^{13} \mathrm{C} \mathrm{NMR}\left(126 \mathrm{MHz}, \mathrm{CDCl}_{3}\right)$<smiles>CC(C)(C)OC(=O)N1CCC(CCc2ccccn2)CC1</smiles>
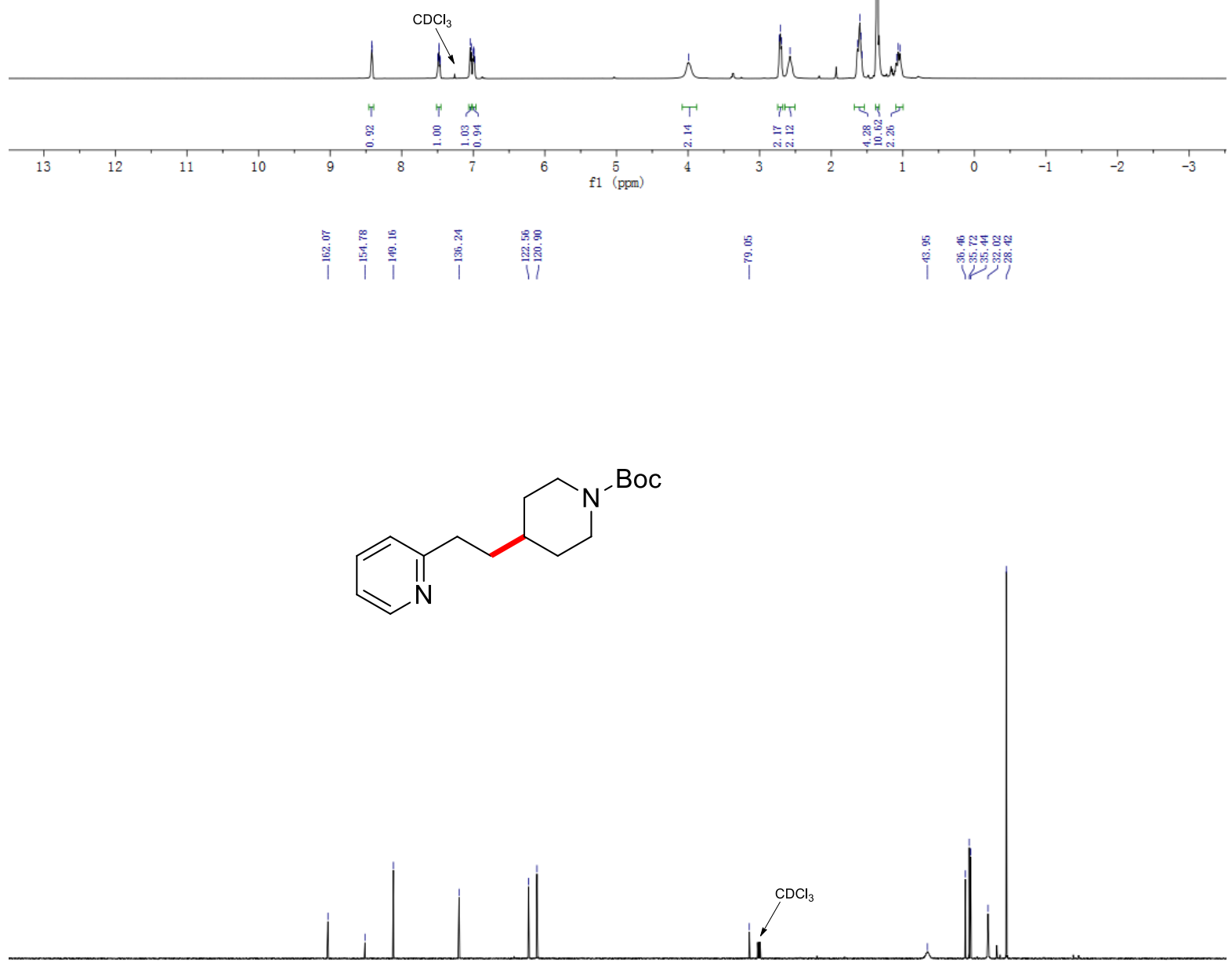

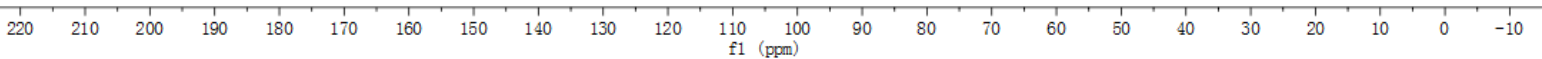


${ }^{1} \mathrm{H}$ NMR $\left(500 \mathrm{MHz}, \mathrm{CDCl}_{3}\right),{ }^{13} \mathrm{C} \mathrm{NMR}\left(126 \mathrm{MHz}, \mathrm{CDCl}_{3}\right)$

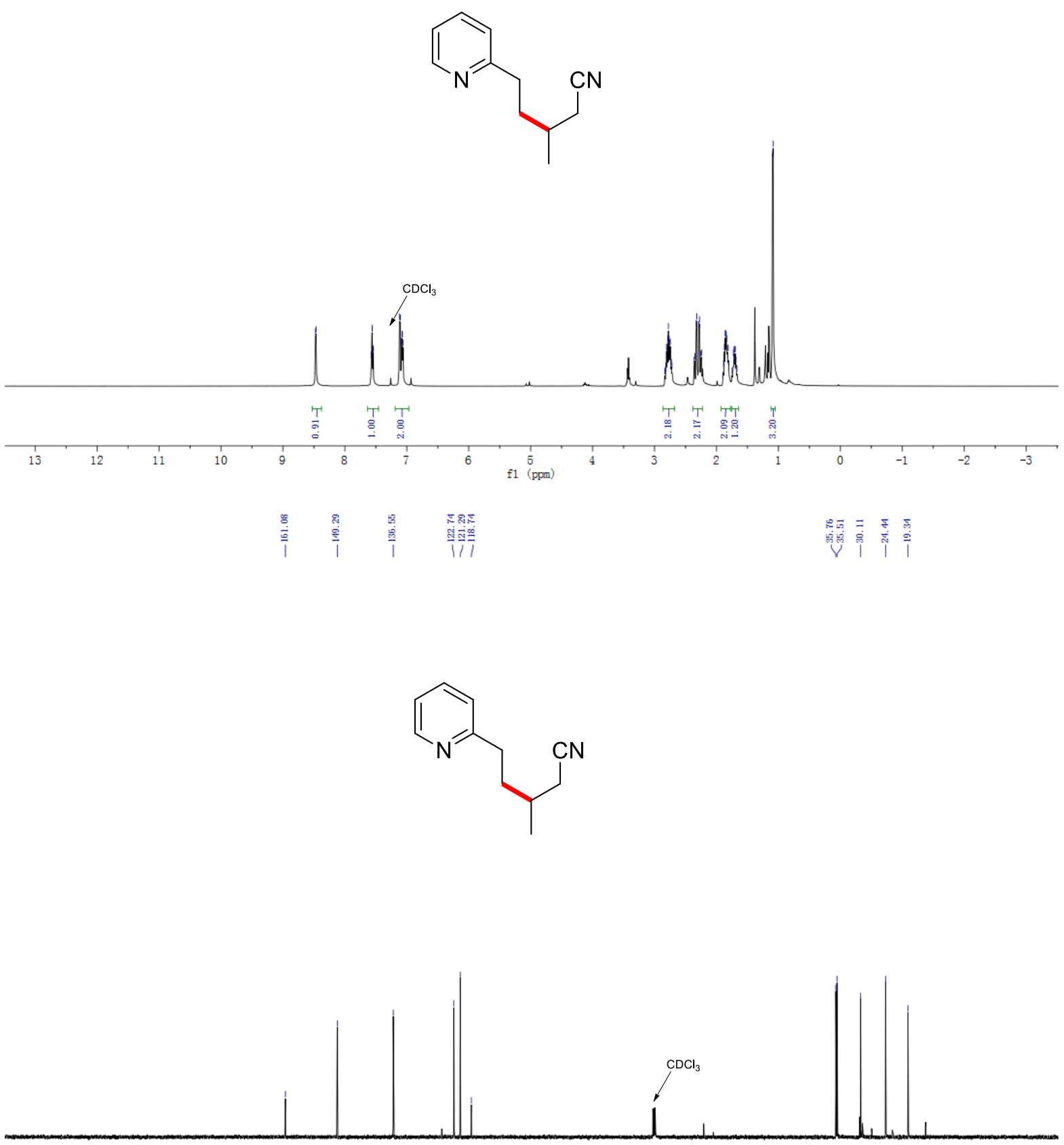

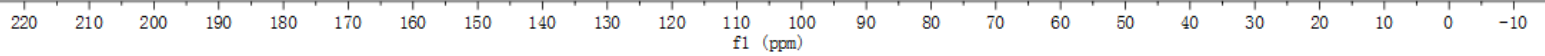


${ }^{1} \mathrm{H}$ NMR $\left(500 \mathrm{MHz}, \mathrm{CDCl}_{3}\right),{ }^{13} \mathrm{C} \mathrm{NMR}\left(126 \mathrm{MHz}, \mathrm{CDCl}_{3}\right)$
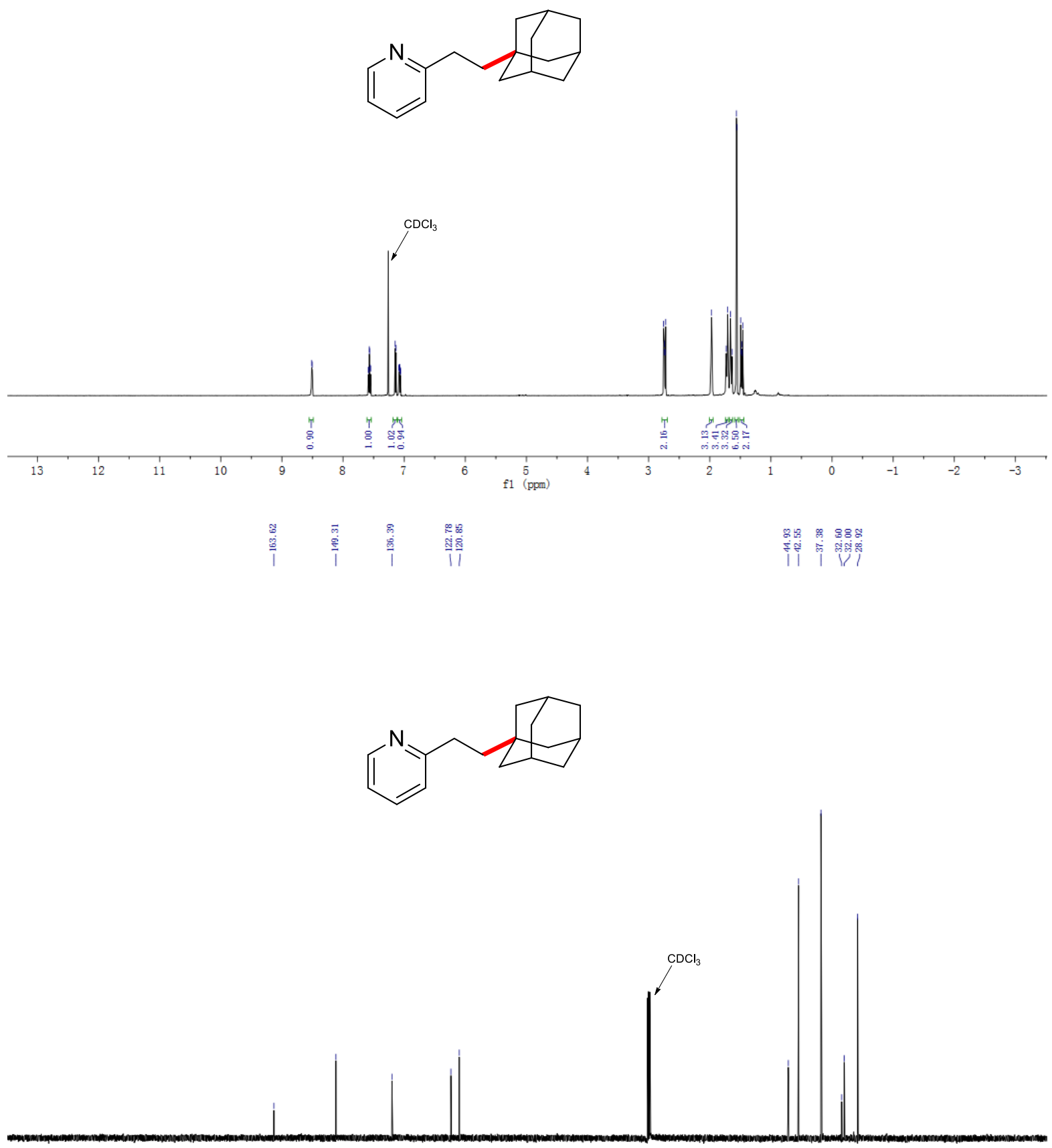

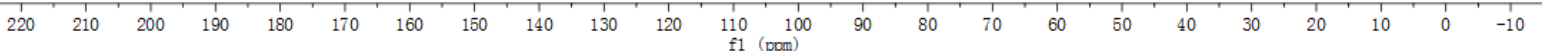


${ }^{1} \mathrm{H}$ NMR $\left(500 \mathrm{MHz}, \mathrm{CDCl}_{3}\right),{ }^{13} \mathrm{C} \mathrm{NMR}\left(126 \mathrm{MHz}, \mathrm{CDCl}_{3}\right)$
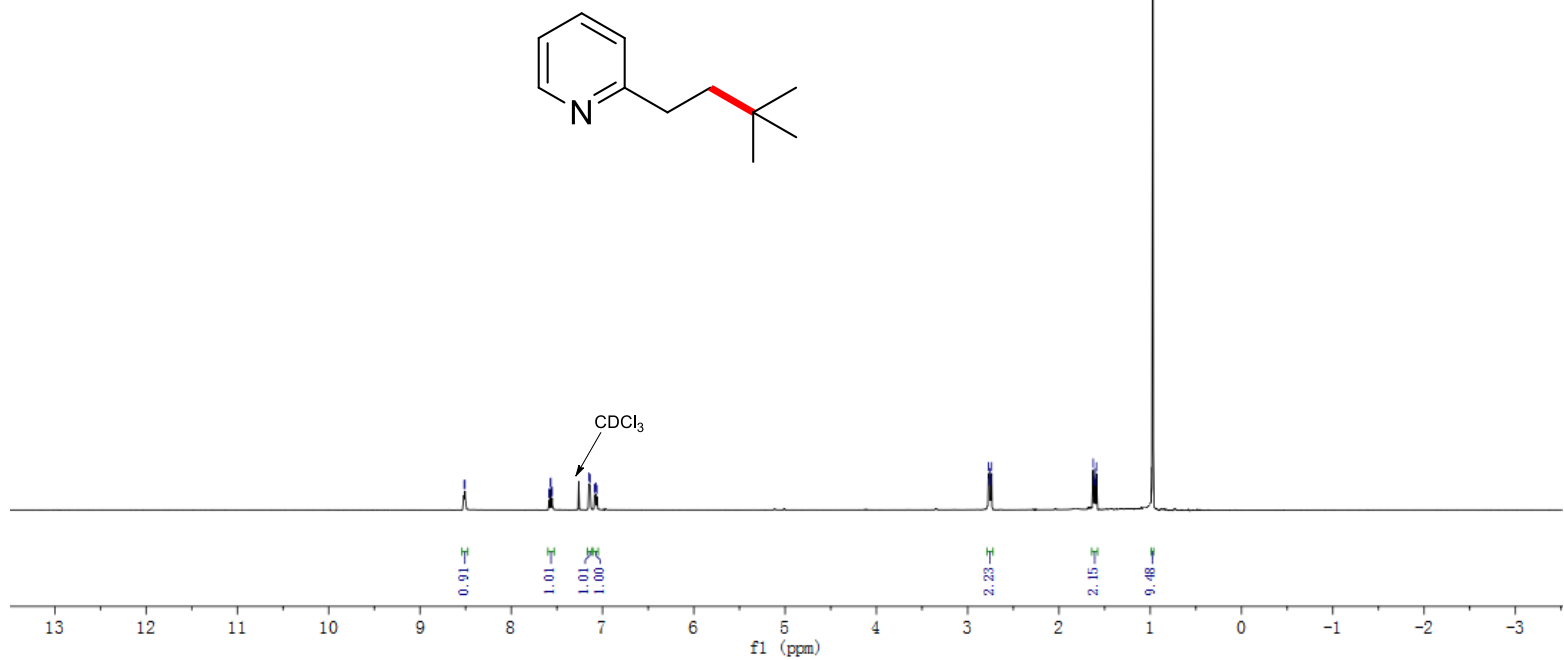

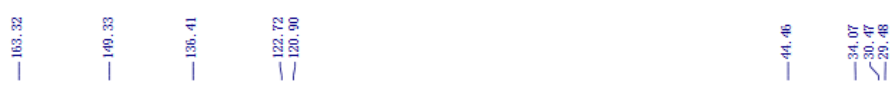
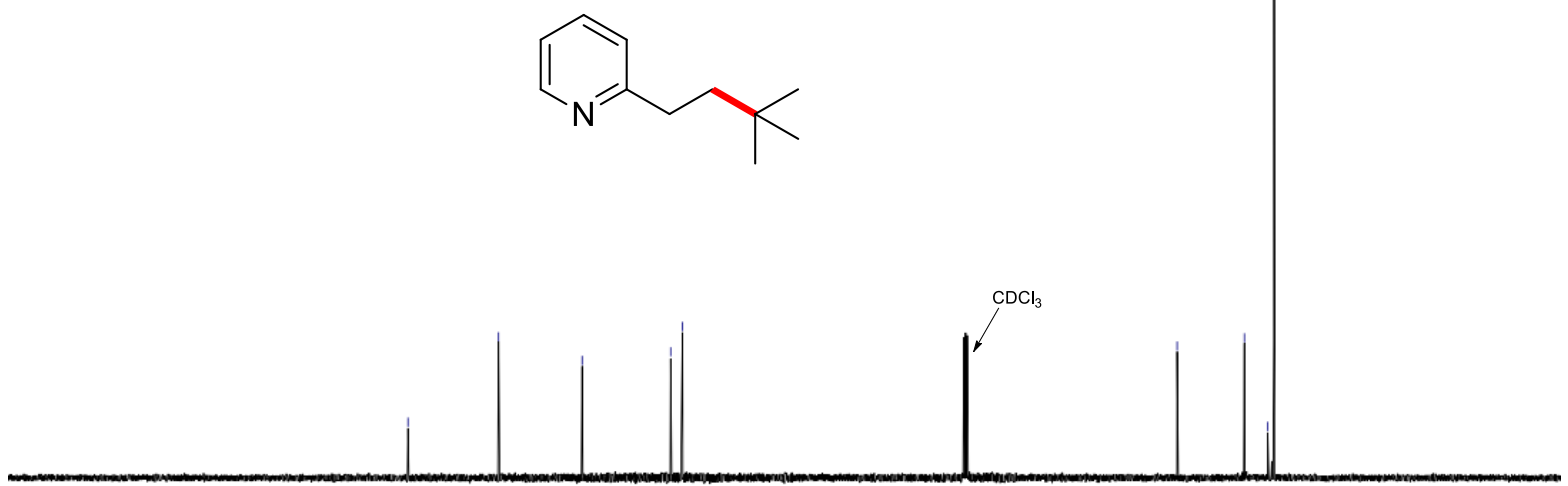

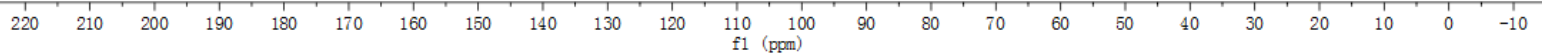




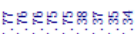

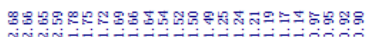

${ }^{1} \mathrm{H}$ NMR $\left(500 \mathrm{MHz}, \mathrm{CDCl}_{3}\right),{ }^{13} \mathrm{C} \mathrm{NMR}\left(126 \mathrm{MHz}, \mathrm{CDCl}_{3}\right)$
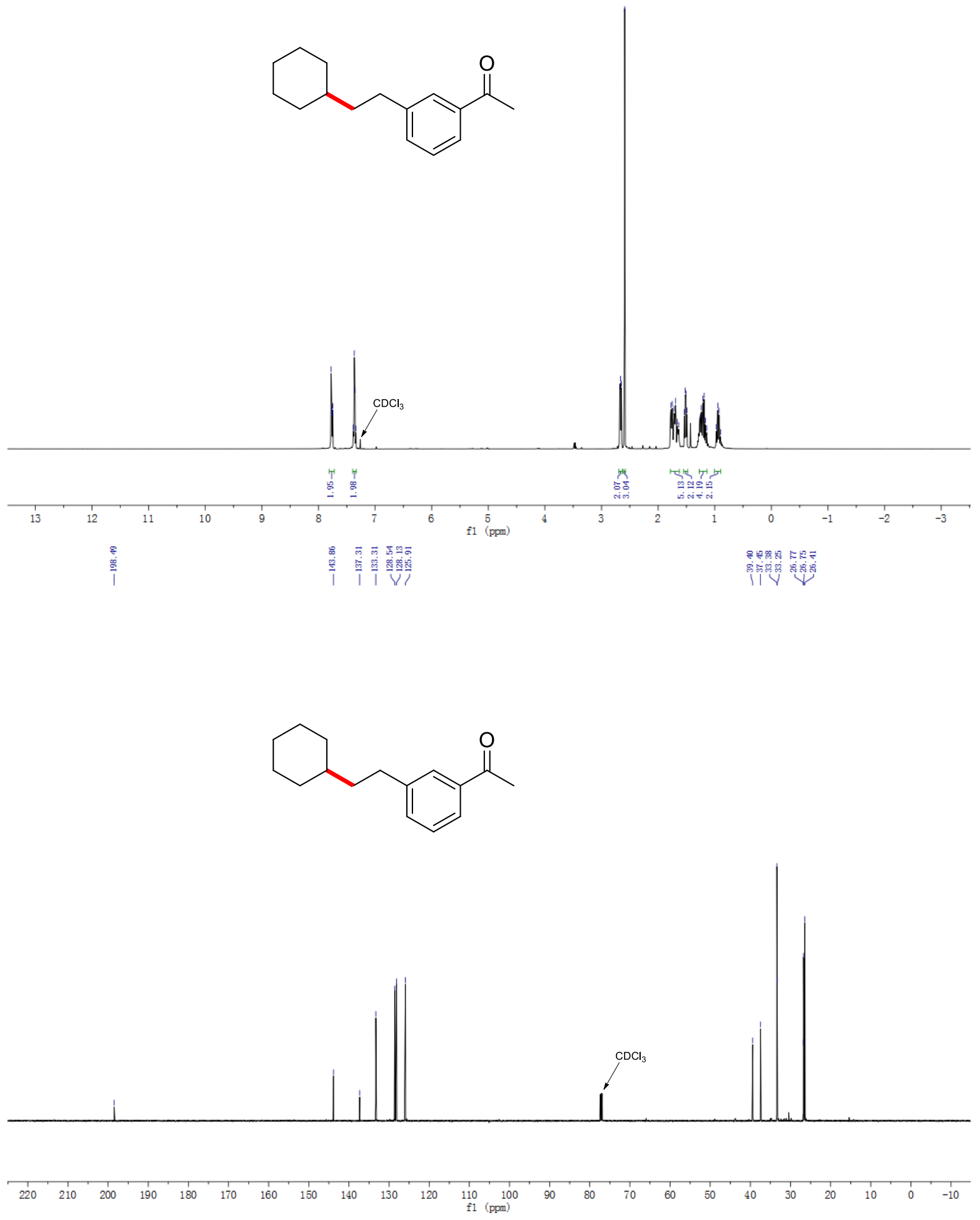

S-117 


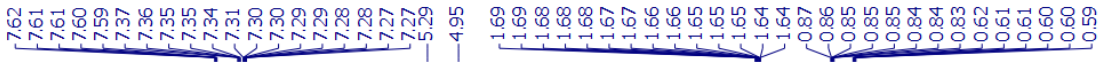

${ }^{1} \mathrm{H}$ NMR $\left(500 \mathrm{MHz}, \mathrm{CDCl}_{3}\right),{ }^{13} \mathrm{C} \mathrm{NMR}\left(126 \mathrm{MHz}, \mathrm{CDCl}_{3}\right)$<smiles>C=C(c1ccccc1)C1CC1</smiles>
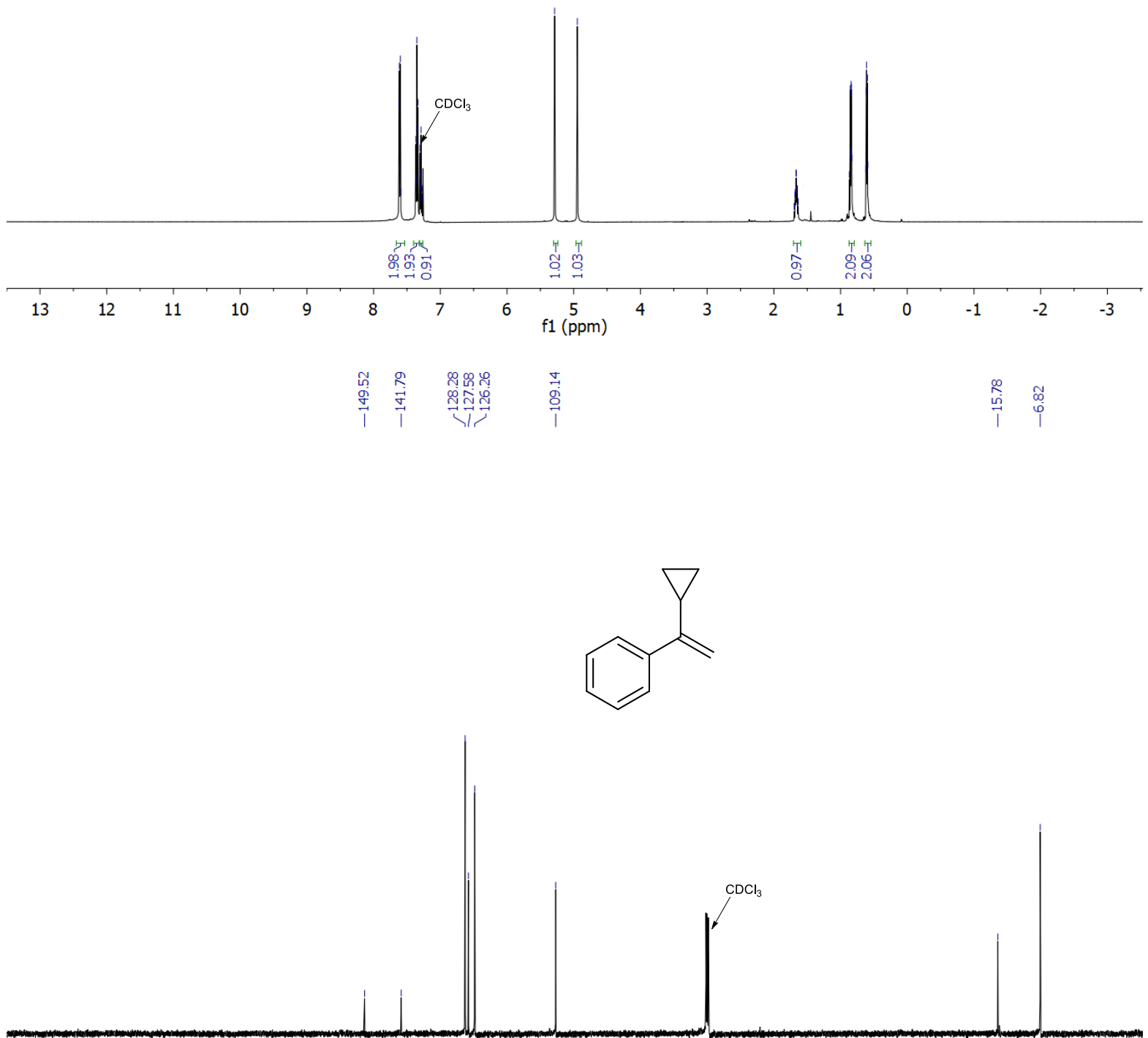

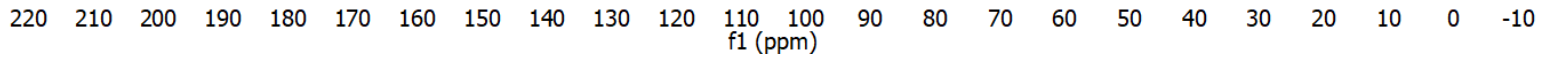


${ }^{1} \mathrm{H} \mathrm{NMR}\left(500 \mathrm{MHz}, \mathrm{CDCl}_{3}\right),{ }^{13} \mathrm{C} \mathrm{NMR}\left(126 \mathrm{MHz}, \mathrm{CDCl}_{3}\right)$

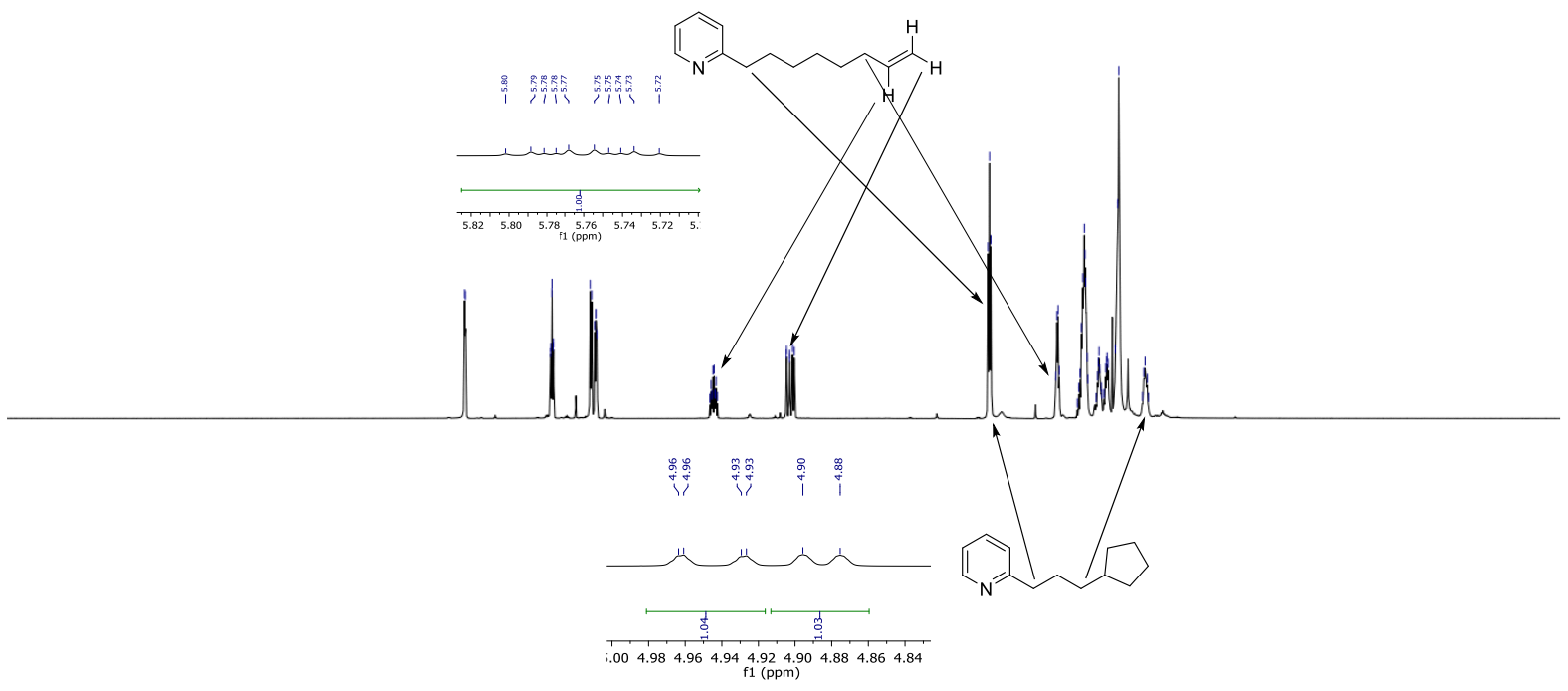

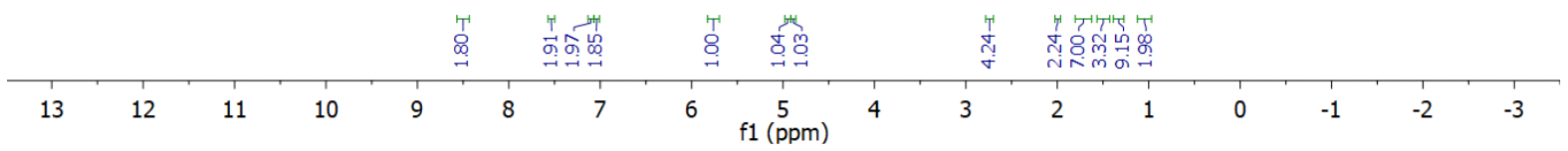

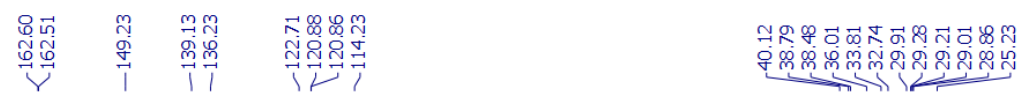
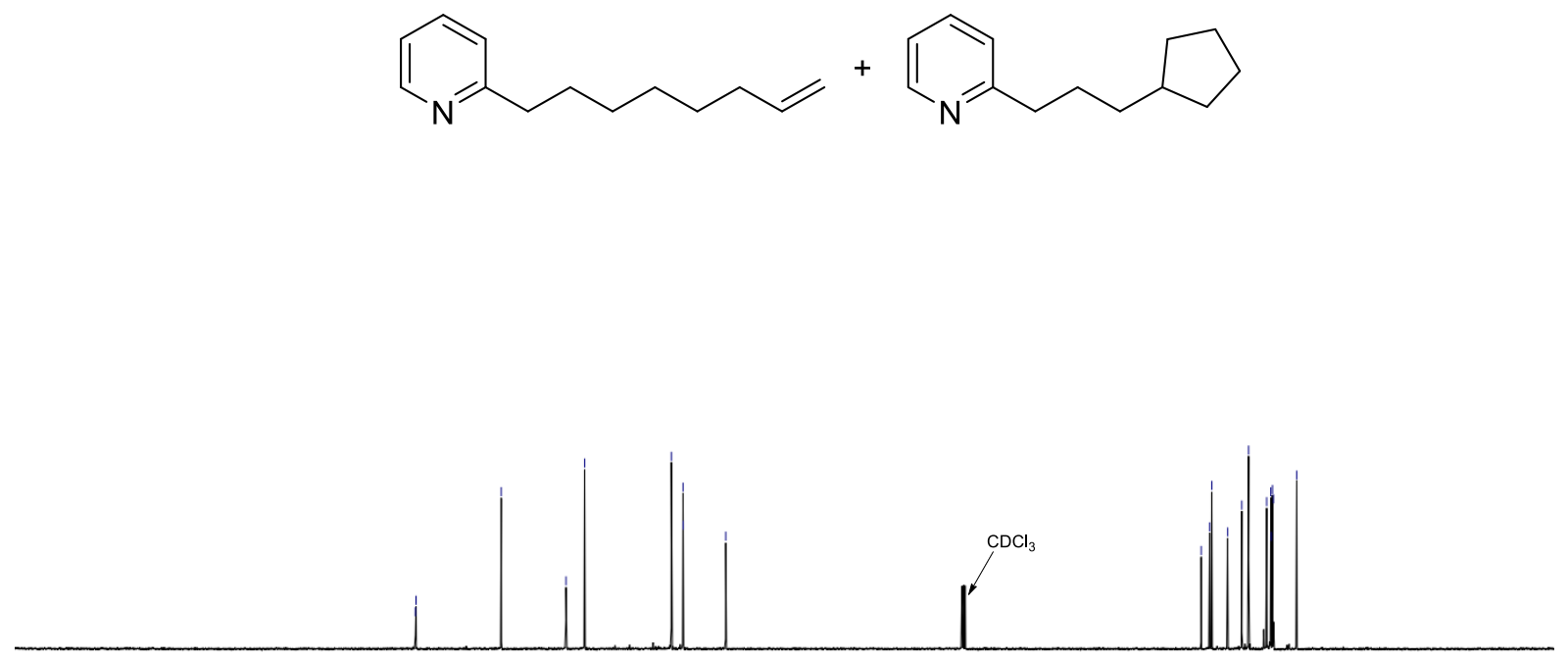

$\begin{array}{llllllllllllllllllllllll}220 & 210 & 200 & 190 & 180 & 170 & 160 & 150 & 140 & 130 & 120 & \begin{array}{c}110 \\ \mathrm{f} 1(\mathrm{ppm})\end{array} & 90 & 80 & 70 & 60 & 50 & 40 & 30 & 20 & 10 & 0 & -10\end{array}$ 
${ }^{1} \mathrm{H}$ NMR $\left(500 \mathrm{MHz}, \mathrm{CDCl}_{3}\right),{ }^{13} \mathrm{C} \mathrm{NMR}\left(126 \mathrm{MHz}, \mathrm{CDCl}_{3}\right)$

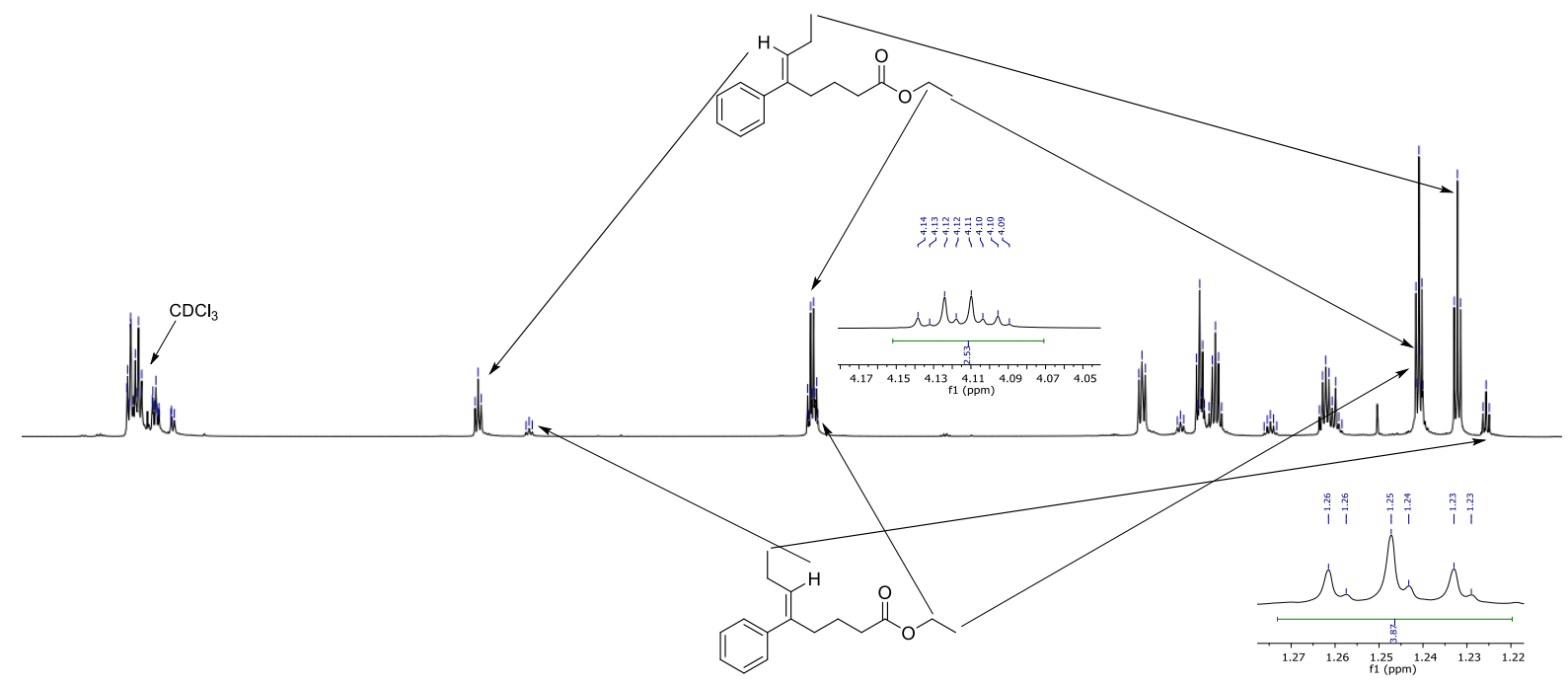

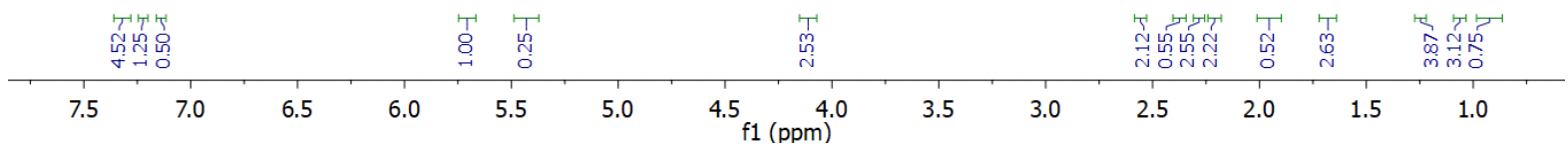

\begin{tabular}{|c|c|c|}
\hline 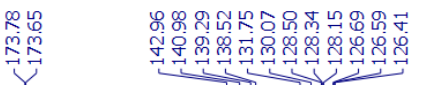 & 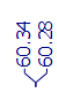 & 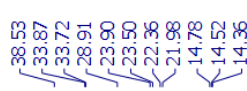 \\
\hline
\end{tabular}<smiles>CC/C=C(/CCCC(=O)OCC)c1ccccc1</smiles>

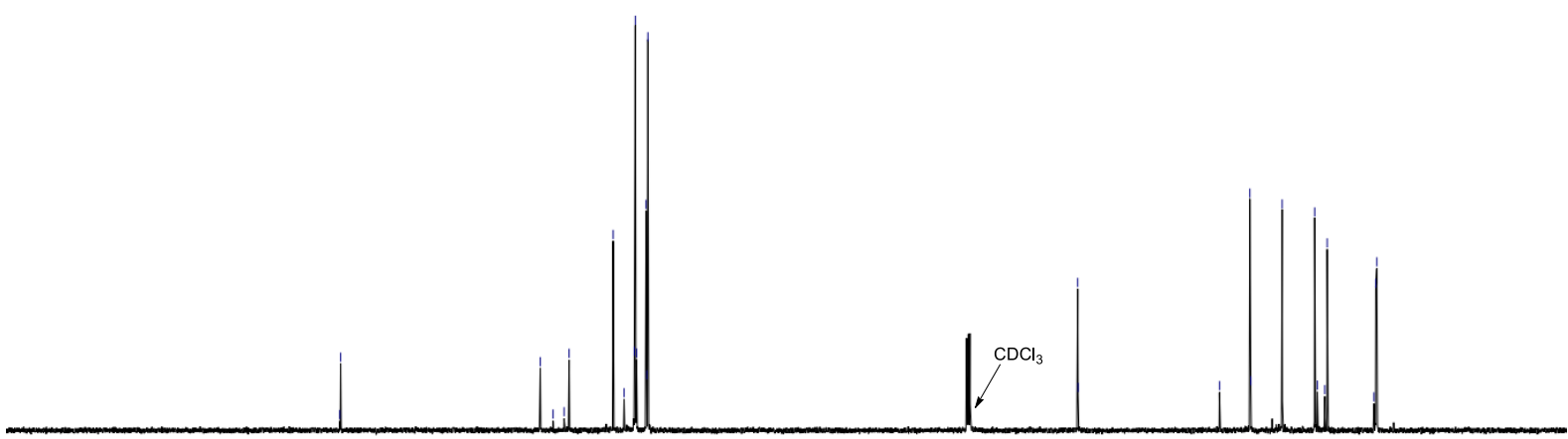


${ }^{1} \mathrm{H} \mathrm{NMR}\left(500 \mathrm{MHz}, \mathrm{CDCl}_{3}\right),{ }^{13} \mathrm{C} \mathrm{NMR}\left(126 \mathrm{MHz}, \mathrm{CDCl}_{3}\right),{ }^{2} \mathrm{H} \mathrm{NMR}\left(61 \mathrm{MHz}, \mathrm{CHCl}_{3}\right)$

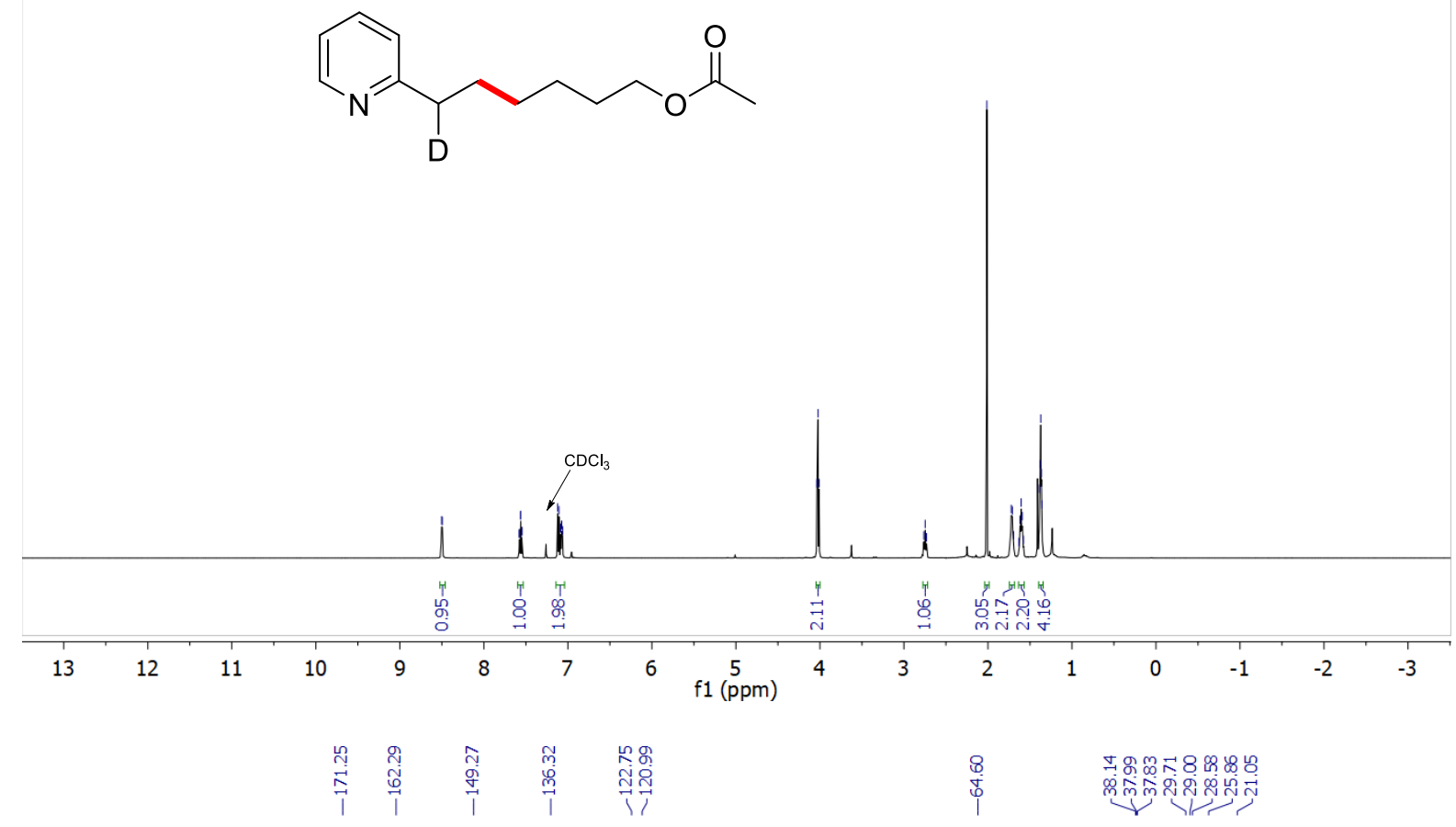<smiles>[2H]C(CCCCCOC(C)=O)c1ccccn1</smiles>

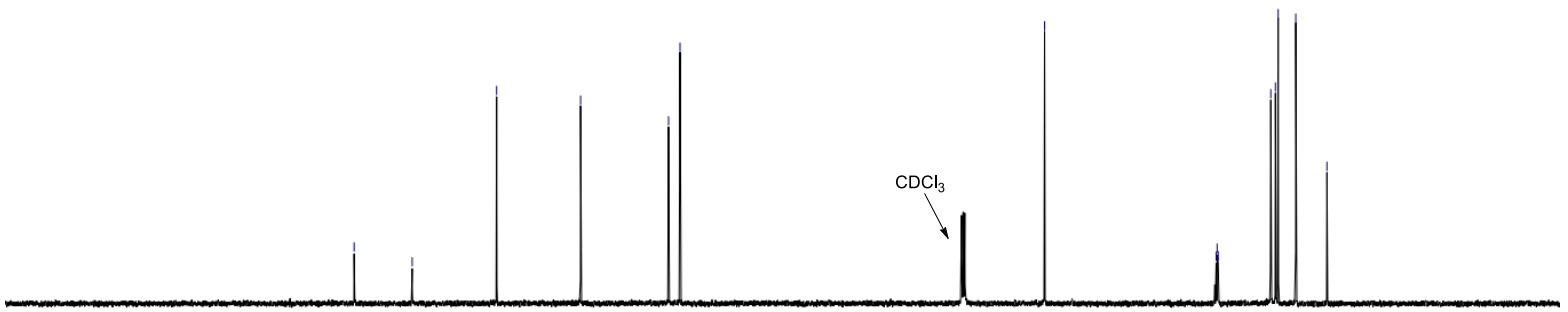

$\begin{array}{llllllllllllllllllllllllllllllll}220 & 210 & 200 & 190 & 180 & 170 & 160 & 150 & 140 & 130 & 120 & \begin{array}{c}110 \\ \mathrm{f} 1(\mathrm{ppm})\end{array} & 100 & 80 & 70 & 60 & 50 & 40 & 30 & 20 & 10 & 0 & -10\end{array}$ 


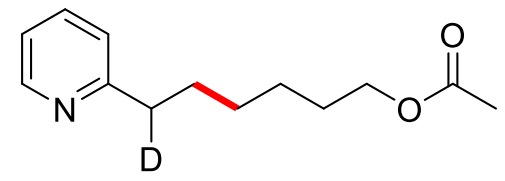

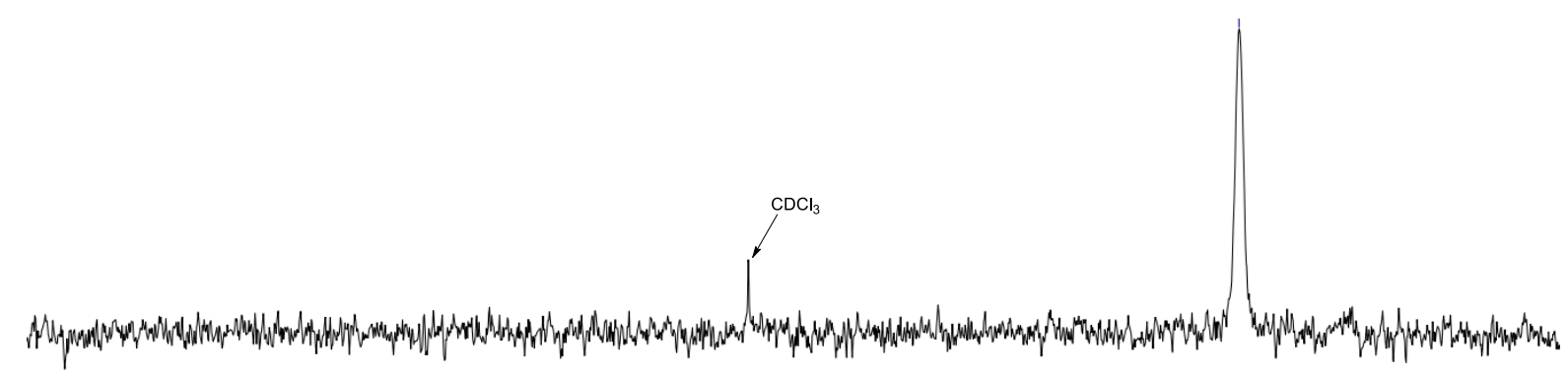

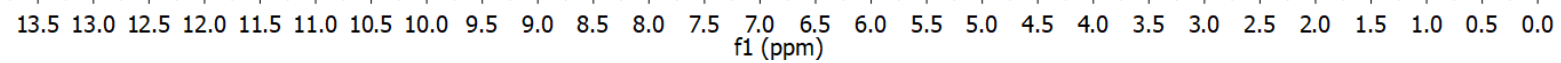

